Hilâl. Studi turchi e ottomani 8

\title{
I dispacci di Francesco Vendramin, ultimo bailo a Costantinopoli (1796-1797)
}

Maria Pia Pedani

a cura di Antonio Fabris

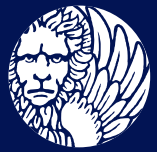

Edizioni

Ca'Foscari 

I dispacci di Francesco Vendramin, ultimo bailo a Costantinopoli (1796-1797)

\section{Hitâl}

Studi turchi e ottomani

Serie diretta da

Elisabetta Ragagnin

8

Edizioni

Ca'Foscari 


\section{Hilâl \\ Studi turchi e ottomani}

\section{Direttori | General editors}

Elisabetta Ragagnin (Freie Universität, Berlin)

\section{Comitato scientifico | Advisory board}

Bülent Arı (TBMM Milli Saraylar, Müzecilik ve Tanıtım Başkanı, İstanbul, Türkiye)

Dejanirah Couto (École Pratique des Hautes Études «EPHE», Paris, France)

Zayabaatar Dalai (Institute for Mongol Studies, National University of Mongolia; National Council for Mongol Studies, Mongolia)

Mehmet Yavuz Erler (Ondokuz Mayıs Üniversitesi, Samsun, Türkiye)

Fabio Grassi ( «La Sapienza» Università di Roma, Italia)

Figen Güner Dilek (Gazi Üniversitesi, Ankara, Türkiye)

Stefan Hanß (University of Cambridge, UK)

Baiarma Khabtagaeva (Szegedi Tudományegyetem, Magyarország)

Nicola Melis (Università degli Studi di Cagliari, Italia)

Melek Özyetgin (Yildiz Üniversitesi, İstanbul, Türkiye)

Cristina Tonghini (Università Ca' Foscari Venezia, Italia)

\section{Direzione e redazione}

Università Ca' Foscari Venezia

Dipartimento di Studi sull'Asia sull'Africa mediterranea

Sezione Asia Orientale e Antropologia

Palazzo Vendramin dei Carmini

Dorsoduro 3462

30123 Venezia

e-ISSN 2610-9484

ISSN 2610-8917

URL http://edizionicafoscari.unive.it/it/edizioni/collane/hilal/ 


\section{I dispacci di Francesco Vendramin, ultimo bailo a Costantinopoli (1796-1797)}

Maria Pia Pedani

a cura di Antonio Fabris

Venezia

Edizioni Ca' Foscari - Digital Publishing 2019 



\title{
I dispacci di Francesco Vendramin, ultimo bailo a Costantinopoli (1796-1797)
}

\author{
Maria Pia Pedani \\ a cura di Antonio Fabris
}

\section{Abstract}

The transcripts of the 55 dispatches written by Francesco Vendramin, the last Venetian bailo of Constantinople between 1796 and 1797, appear very important to the eye of the historian. Even though they were written in Constantinople, they reflect the hardships of the political climate that the fall of the Veneta Repubblica and the establishment of the Municipalità Provvisoria brought to Venice.

Moreover, they provide a unique insight into the bailo house in Constantinople. Vendramin had to explain the functioning of the bailaggio and the necessity of the diplomatic office to maintain a decorum of credibility for the State (both the Repubblica and the Municipalità). This needed to be clarified to the new rulers, who were mostly bourgeois and not experts in political issues, especially issues of an international nature, while the old rulers, the august senators, have been experts for decades in both internal and external political affairs of the Republic. The first 27 dispatches were written when the Veneta Repubblica was still alive. The remaining 28 were written after its fall (12th May 1797), when Vendramin had no official role. He was accredited with the Porta Ottomana, as the Venetian delegate of the Doge, but he never received any formal task by the Municipalità. Nevertheless the Ottoman government continued to consider Vendramin as an ambassador, not knowing how to act otherwise.

The first collection of dispatches again proposes, with proper adjustments to the new situation, the schemes and the themes that characterize the VenetianOttoman relationship in the modern age. The second group is full of information on the Venetian colony in the Empire. As a consequence, it gives information on the functioning of the consulates and on the personal licenses given to merchants and vendors. Moreover, the figure of the French ambassador du Bayet appears strong. He stands as a supporter of many choices in the name of an effective French supremacy on Venice, that in Constantinople is shown without the shield of the Municipalità.

Keywords Francesco Vendramin (1751-1818). Bailo. Constantinople. Republic of Venice. Municipalità Provvisoria. 
I dispacci di Francesco Vendramin, ultimo bailo a Costantinopoli (1796-1797)

Maria Pia Pedani, Antonio Fabris

(C) 2019 Maria Pia Pedani, Antonio Fabris per il testo

(c) 2019 Edizioni Ca' Foscari - Digital Publishing for la presente edizione

\section{(). (1)}

Quest'opera è distribuita con Licenza Creative Commons Attribuzione 4.0 Internazionale This work is licensed under a Creative Commons Attribution 4.0 International License

\section{OPEN}

Qualunque parte di questa pubblicazione può essere riprodotta, memorizzata in un sistema di recupero dati o trasmessa in qualsiasi forma o con qualsiasi mezzo, elettronico o meccanico, senza autorizzazione, a condizione che se ne citi la fonte.

Any part of this publication may be reproduced, stored in a retrieval system, or transmitted in any form or by any means without permission provided that the source is fully credited.

Edizioni Ca' Foscari - Digital Publishing

Università Ca' Foscari Venezia

Dorsoduro 3246, 30123 Venezia

http://edizionicafoscari.unive.it/

ecf@unive.it

1a edizione dicembre 2019

ISBN 978-88-6969-372-4 [ebook]

ISBN 978-88-6969-373-1 [print]

I dispacci di Francesco Vendramin, ultimo bailo a Costantinopoli (1796-1797) / Maria Pia Pedani, a cura di Antonio Fabris - 1. ed. - Venezia: Edizioni Ca' Foscari Digital Publishing, 2019. - 168 pp.; 23 cm. - (Hilal; 8). - ISBN 978-88-6969-373-1.

URL https://edizionicafoscari.unive.it/it/edizioni/libri/978-88-6969-373-1

DOI https://doi.org/10.30687/978-88-6969-372-4 
I dispacci di Francesco Vendramin, ultimo bailo

a Costantinopoli (1796-1797)

Maria Pia Pedani

a cura di Antonio Fabris

\section{Sommario}

Premessa

Introduzione

Antonio Fabris

Dispacci

Elenco dei dispacci

Nota degli allegati ai dispiacci di Francesco Vendramin 

In memoria di

Maria Pia Pedani (1952-2019) 



\section{Premessa}

Questo volume è prima di tutto un omaggio.

Un omaggio a Maria Pia per il suo grande impegno nella ricerca e nella didattica. Un omaggio a una donna che ha saputo mirabilmente coniugare gli impegni familiari con il lavoro. Un omaggio a una persona discreta e appassionata, che è riuscita a coinvolgere e farsi amare dagli studenti. Un omaggio a una studiosa che ha saputo farci immergere con i suoi scritti nel mondo passato, riuscendo a farcelo conoscere e vivere come presente.

Maria Pia è stata l'ideatrice e fondatrice di questa collana di studi turchi e ottomani ed era giusto che questa sua ultima ricerca uscisse per Edizioni Ca' Foscari e nella 'sua' collana.

Dopo aver dato alle stampe le relazioni ancora inedite dei baili veneziani a Costantinopoli, voleva rendere fruibili agli studiosi anche i dispacci che i baili inviarono a Venezia per oltre quattro secoli. In questo progetto si respirava l'aria del suo passato di archivista (lavorò infatti presso l'Archivio di Stato di Venezia dal 1979 al 1999) sia per l'attenzione volta alla conservazione dei documenti, che una volta editati vengono spostati con minor frequenza, sia l'aria dell'insegnante che voleva capire e far capire quali furono i reali rapporti tra Venezia e Costantinopoli in età moderna, ancor oggi spesso mistificati nell'agone politico.

La scelta di iniziare questo immane progetto dalla fine - Francesco Vendramin fu infatti l'ultimo bailo veneziano a Costantinopoli, operando dal 1796 alla caduta della Repubblica nel 1797 - trova ragione nella pubblicazione integrale dei dispacci precedenti (Dispacci da Costantinopoli di Ferigo Foscari (1792-1796), a cura di Franca Cosmai e Stefano Sorteni, Venezia: La Malcontenta, 1996, 2 voll.). Si voleva evidentemente offrire un quadro completo della fine di questa istituzione. 
Maria Pia non ha fatto in tempo a scrivere l'introduzione al volume, lasciando solo pochi appunti. Così è stata fatta la scelta di lasciare questo materiale al grezzo, offrendo più che mai la possibilità agli studiosi che leggeranno i dispacci di inserirli nel giusto contesto e trarne tutte le possibili indicazioni. Solo una breve introduzione darà una sistemazione organica agli appunti per illustrare le caratteristiche principali di questi dispacci.

Elisabetta Ragagnin

Antonio Fabris 


\section{Introduzione}

\section{Antonio Fabris}

Università Ca' Foscari Venezia, Italia

Francesco Antonio Maria Vendramin fu l'ultimo bailo della Repubblica di Venezia a Costantinopoli.

Figlio di Pietro e di Fiorenza Ravagnan, nacque a Venezia il 5 luglio 1751 nell'avito palazzo in parrocchia di Santa Maria del Carmelo, vulgo dei Carmini, oggi sede della presidenza e degli uffici del Dipartimento di Studi sull'Asia e dell'Africa Mediterranea dell'Università Ca' Foscari.

La sua carriera politica iniziò a 25 anni, con l'elezione a Savio agli ordini, il primo incarico per i giovani che volevano fare carriera politica. Appare difficile ricostruire tutto il suo cursus honorum perché talvolta nei registri del Segretario alle Voci, l'ufficio che registrava la successione delle diverse cariche politiche a cui la nobiltà era eletta, manca il patronimico cosicché è difficile distinguere se il «Francesco Vendramin» registrato dal Segretario è il nostro oppure un omonimo, figlio di Giovanni Vendramin, suo coetaneo. Comunque lo sappiamo alternativamente eletto tra il 1783 e il 1791 tra i Cinque Savi di Terraferma e il Savio Cassier; quindi fu per tre volte tra i Sei Savi del Collegio e tra i Tre inquisitori sopra l'amministrazione dei pubblici ruoli e alle cose del Levante, Dalmazia e Albania. Fu comunque tra i Savi di Terraferma, magistratura incaricata di sovrintendere all'esercito veneto, che il nostro si distinse, scrivendo argute e precise memorie sullo stato delle armate venete e sui possibili rimedi per adeguarle ai tempi.

Fu il buon credito politico guadagnato nei tanti uffici che convinse il Senato ad eleggerlo il 14 settembre 1794, a soli 43 anni, quale nuovo bailo a Costantinopoli e successore di Ferigo Todero Foscari, 
ambasciatore anziano e di provata esperienza chiamato a coronare la sua carriera nell'importante e delicata sede stanbuliota.

Nel frattempo si era sposato il 24 settembre 1771 con Alba Corner di Andrea. I Corner della Ca' Grande a San Maurizio erano una famiglia assai ricca e il padre Andrea fu un grande protettore di artisti, tra cui Goldoni e Bettinelli, mentre il palazzo fu frequentato, tra i tanti, da Vittorio Alfieri, Ippolito Pindemonte e Ugo Foscolo. Palazzo Corner è oggi sede della Prefettura, ma si tratta di un altro edificio perché l'originario cinquecentesco fu distrutto in un incendio. Probabilmente per Francesco fu un matrimonio di convenienza, versando la sua famiglia in condizioni economiche non particolarmente floride, anche se bisogna notare una comunanza di ideali progressisti: Alba fu certamente uno spirito libero, e sia suo fratello Nicolò, detto Nicoletto, sia Francesco Vendramin, furono massoni e di idee giacobine. Nicolò, che fu anche un giocatore incallito, fu il primo presidente della Municipalità Provvisoria, e giocò certamente un certo ruolo nella permanenza di Vendramin a Costantinopoli dopo la caduta della Repubblica.

Contrariamente alle leggi venete, Alba raggiunse il marito nella missione costantinopolitana. Non ci sono notizie dirette del viaggio, ma abbiamo notizia che Pindemonte si rifiutò di accompagnarla e trovò la disponibilità del bolognese Bondioli. Si imbarcò per Venezia il 30 agosto 1797, assieme al vecchio bailo Foscari, e approdò al lazzaretto il 6 ottobre. Dal matrimonio nacquero due figlie, Fiorenza, che si suicidò venticinquenne dopo un disastroso matrimonio, e Maria, che invece visse felicemente circondata da numerosa prole.

Di Vendramin non abbiamo molte notizie dopo il suo rientro da Costantinopoli: con lettera del 14 febbraio 1816 chiese all'I.R. Commissione araldica di Milano la conferma del titolo baronale, già conferitogli dal Regno d'Italia, mentre il 30 aprile dell'anno successivo chiese la conferma dell'antica nobiltà veneta e, poiché i due titoli non erano cumulabili, optò per il titolo avito.

Morì nel 1818. Di lui ci rimane solo una breve memoria di un bisnipote che scrisse «Ottimo cavaliere il nonno di mia madre, ma forse trascurato».

Francesco Vendramin scrisse in tutto 55 dispacci, dal 22 aprile 1796 al 25 novembre 1797. Questi sono stati conservati per oltre un secolo e mezzo nella serie «Senato, Dispacci ambasciatori e residenti, Costantinopoli», fino a quando, il 10 giugno 1958, come si legge in un appunto di mano della dott.ssa Maria Francesca Tiepolo, già direttrice dell'Archivio di Stato di Venezia, gli ultimi 28 dispacci sono stati traslati nel fondo archivistico «Municipalità provvisoria».

Nella logica archivistica si tratta di un'operazione coerente in quanto mutando l'entità statale di riferimento le carte da questo 
prodotte, o ad esso indirizzate, devono conservarsi in archivi diversi da quelle emesse in nome dello stato precedente. Dunque poiché Vendramin, bailo a Costantinopoli per la Repubblica di Venezia, dopo la caduta di questa (12 maggio 1797) rappresentò la Municipalità Provvisoria, nonostante nessun incarico formale in questo senso e dunque nessun nuovo accreditamento presso la Porta ottomana, che comunque nel dubbio e nell'incertezza della situazione in Europa continuò a considerarlo ambasciatore di Venezia, nonostante ufficialmente non avesse più alcuna veste diplomatica, i dispacci ad essa indirizzati devono essere conservati nella documentazione di questa.

Inoppugnabile logica archivistica, che però ha spezzato l'unità storica della documentazione che, pur prodotta dallo stesso soggetto, nel medesimo luogo e con la stessa funzione, viene oggi conservata in due fondi archivistici diversi.

Da notare, infine, che la scelta di dividere la documentazione prodotta dagli ambasciatori veneziani in servizio presso le corti estere è stata operata solo per i dispacci del bailo a Costantinopoli, mentre i dispacci di Vienna, Londra... mantengono la loro posizione tra le carte dello Stato veneto.

I dispacci del bailo Francesco Vendramin offrono un interessante spaccato della vita nella casa bailaggia, oggi sede stanbuliota dell'ambasciata d'Italia in Turchia, a cavallo tra gli ultimi mesi di vita della Veneta Repubblica e i primi della Municipalità Provvisoria.

Il primo gruppo di 27 dispacci furono infatti scritti da Vendramin, spesso firmati anche dal bailo uscente che rimase a lungo a Istanbul per la difficoltà di ottenere le credenziali e lasciare la città, in qualità di bailo. Si tratta di scritti sostanzialmente in linea con quanto da secoli era già avvenuto, pur ovviamente legati a circostanze precise: la descrizione dell'imbarco ufficiale, che anticipava di settimane o mesi della vera partenza; del viaggio, funestato da numerosi contrattempi: non solo i venti contrari, ma anche il richiamo, mentre il bailo col suo seguito era già a Corfù, della fregata armata che doveva condurlo a Costantinopoli in quanto il Senato volle radunare la flotta a protezione della città lagunare. Così, Vendramin, lasciato Venezia il 19 maggio 1796, approdò nel Corno d'Oro oltre sei mesi dopo, il 25 novembre. Seguono tutte le indicazioni relative alla cerimonia di accreditamento, alle regole di protocollo, alla distinzione data ai diversi rappresentanti diplomatici europei e alla considerazione che la Porta dimostra di avere per la figura del bailo veneziano.

Non mancano i problemi con i pirati algerini (ufficialmente Venezia era in guerra col cantone d'Algeri, anche se non fu una guerra combattuta e che, ereditata dapprima dai francesi e quindi dagli im- 
periali che uno dopo l'altro si succedettero alla Veneta Repubblica, terminò nel nulla), con le differenti esenzioni doganali che ogni stato cerca di strappare alla Porta e una serie di tematiche che, pur variando nei nomi e nelle situazioni, ripropongono episodi già visti nello scorrere dei secoli.

Unico appare invece quanto seguì all'increscioso incidente accaduto a Smirne il 12 marzo 1797. Un greco, suddito veneto di Cefalonia, ma nell'elenco dei protetti dalla corte zarista, uccise un giannizzero e i suoi compagni, non riuscendo a catturare il colpevole, appiccarono il fuoco al quartiere europeo, saccheggiando case e magazzini e non rispettando nemmeno i privilegi diplomatici assalendo, oltre alle abitazioni civili, anche le sedi consolari, tanto che la popolazione poté trovare riparo solo a bordo delle navi ancorate in porto. La questione andò via via crescendo per le proteste accolte dal gran visir e dal sultano, che imputarono ai sudditi veneti, e ai loro rappresentanti consolari e diplomatici, ogni colpa, rimettendo in discussione le capitolazioni e arrivando a decidere per l'espulsione dei greci di Zante, Cefalonia e Corfù, nonostante il loro status di sudditi veneti.

L'ultimo dispaccio di questo gruppo indirizzato al doge, datato 10 giugno 1797, è un misto di preoccupazione e fiducia nel futuro, con una pressante richiesta di istruzioni perché nessuno mai si era trovato in una simile situazione.

Il secondo gruppo di dispacci inizia con la missiva datata 20 giugno. Il dispaccio è indirizzato ai «Cittadini Municipalisti», e venne spedito via ambasciata di Francia. In questo appare evidente come Vendramin ponga al centro della sua missione il servizio alla Patria, indipendentemente dal governo in carica. Questo secondo gruppo di dispacci è per molti versi più interessante e permette di guardare dentro la casa bailaggia e di avere informazioni sul personale come mai prima. Infatti finché visse la Veneta Repubblica i senatori che ascoltavano la lettura dei dispacci sedevano sui loro scranni a compimento di una lunga carriera che li aveva portati a conoscere, in maniera diretta o indiretta, molti particolari inerenti le sedi diplomatiche e il loro personale. Al contrario gran parte dei municipalisti, proveniente per lo più dalla borghesia cittadina, erano affatto digiuni di queste informazioni e dunque, per permettere loro di comprendere la situazione, Vendramin si sofferma su dei particolari dati altrimenti per scontati. Sappiamo così dell'ortolano, dei cocchieri, del «guardafuoco», dei sei portantini, dei tre portinai e dei dieci vogatori dei caicchi e della cavana ove trovavano riparo, nonché della disposizione di alcune stanze del palazzo, anche se, bisogna ricordare, l'attuale Palazzo di Venezia è stato riadattato più volte tra Otto e Novecento, mutando completamente la fisionomia dai tempi di Vendramin. 
Si pone innanzitutto il problema dell'avviso del cambio di regime e del riconoscimento del nuovo. In questa fase, come in altre successive, l'ambasciatore di Francia, Jean-Baptiste Aubert du Bayet, la cui ambasciata era confinante, come del resto ancor oggi, sembra il vero burattinaio: tutto avviene su suo consiglio o sotto la sua supervisione. Così la cerimonia nella casa bailaggia avviene sotto gli occhi compiaciuti del francese. Dopo i discorsi di rito, inizia la distribuzione delle coccarde tricolori che, d'ora in avanti, dovranno caratterizzare i sudditi veneti, anche se ben presto si rimarca un uso improprio da parte di molti che, pur non veneti, indossano la coccarda per essere considerati tali e godere di eventuali privilegi ancora accordati ai sudditi di Venezia o dai ducalisti, come venivano chiamati mercanti e negozianti che, pur non essendo sudditi veneti, rientravano comunque tra le persone protette dalla Repubblica a seguito di apposite ducali.

È sempre du Bayet a proporre aiuti e suffragi ai marinai veneti: la guerra col cantone d'Algeri ha reso insicure le rotte e i capitani hanno disarmato le navi nel porto lasciando le ciurme a terra e affamate. Ed è appunto l'ambasciatore francese che si impegna a noleggiare una nave per imbarcare i marinai e dar così loro sostentamento.

Vendramin è sempre più pressante nella richiesta di direttive e di aiuto finanziario. In effetti la sovranità della Municipalità provvisoria si riduce alla sola città di Venezia, così manca di una visione politica di ampio respiro, mancano le risorse finanziarie e la sopravvivenza viene a mala pena raggiunta con un cospicuo aumento della tassazione ai cittadini. Così mancano soldi da spedire, così a Costantinopoli come altrove, e il bailo si vede costretto a licenziare gran parte del personale, mantenendo solo quello strettamente indispensabile.

Una sorte simile tocca anche ai «giovani di lingua», come erano chiamati gli studenti, veneziani o sudditi veneti, che frequentavano la scuola di lingua ottomana attiva da secoli nel palazzo, per prepararsi a diventare dragomanni (interpreti) e consoli veneti. I «giovani di lingua» sono sempre stati un vanto della casa bailaggia e la scuola fu di esempio per altre nazioni. Eppure, in questi mesi tumultuosi, Vendramin si vede costretto a non ospitare né cibare gli studenti che hanno casa in città e a ridurre al minimo le spese per coloro che dimoravano presso il palazzo, riducendoli quasi alla fame, e costringendoli a scrivere un'accorata supplica ai municipalisti.

La professoressa Pedani ha voluto che tutti i suoi libri di studio andassero agli studenti, per cui saranno a breve depositati nel bookcrossing attivo presso la biblioteca del Dipartimento a Palazzo Cappello; similmente, voleva che questa sua ultima fatica fosse di stimolo per gli studenti ad approfondire l'argomento, per cui si sono qui volutamente tralasciati i numerosi episodi inerenti la comunità vene- 
ta che accaddero a Costantinopoli in quei mesi tumultuosi, le fatiche del bailo per contenere i pericoli e una dettagliata descrizione delle perorazioni del Vendramin, utile specchio che, pur da Costantinopoli, riflette le angustie della Venezia di quei mesi, con l'invito a leggere questi dispacci e coglierne le sfumature, studiarli e apprezzare la viva intelligenza di chi li redasse: Francesco Vendramin, un vero spirito nobile veneziano, al di là del regime politico del momento. 


\section{Dispacci}

(Con una inserta)

Le provide disposizioni del decreto 2 corrente posero in grado la mia obbedienza di trasferirmi a bordo della grossa fregata Fama, che col presente punto di acqua si ridusse nel Canale di Fisolo, da dove ho l'onore di segnare questo primo ossequioso dispaccio. Ad accelerare il momento del mio imbarco contribuirono le avvertenti misure prese dal fervoroso impegno de' rispettivi Uffizi e Magistrati, non che l'utile cooperazione del nobil uomo governator di nave ser Zaccaria Bonlini, fornito di cognizione e di sperienza per il prestato servigio sul mare, con piena approvazione dell'eccellentissimo capitan estraordinario delle navi cavalier e procurator Emo di gloriosa ricordanza. Mi lusingo quindi che se non vi si opponga la contrarietà de' tempi potrò approfittare del successivo punto di acqua per passar sopra porto e sciogliere in seguito le vele con sollecitudine, ben sicuro che non lascerà di continuare ad adoprarsi a questo effetto la zelante attività dell'eccellentissimo Magistrato dell'armar con la somministrazione degli occorrenti metodici trabacoli per fornir la nave del restante corredo e dell'indispensabile alimento dell'equipaggio, implorando perciò da vostre Eccellenze i presidi di cui abbisognasse il magistrato medesimo, onde non perdere la stagione favorevole per la celerità del viaggio.

Destinati dall'autorità dell'eccelso Consiglio di dieci per segretario il fedelissimo Francesco Alberti, il quale nelle varie interne incombenze in cui fu impiegato diede continui saggi della sua distinta capacità, e per coadiutore il fedelissimo Sebastian Rizzi, che dimostra tutto il genio per meritarsi la pubblica approvazione, mi riprometto ch'eglino saranno per prestare il più utile servizio. 
Eguale me lo attendo altresì dal fedel Federico Camarata che dal peculiare eccellentissimo Magistrato dei revisori regolatori alla scrittura mi venne comesso per ragionato della carica avendo esso, dietro i principi ereditati dal benemerito suo padre, dato sempre prova di onor e di abilità nelle sostenute ispezioni.

A tenore del decreto 16 maggio 1795, mi seguirà come dragomanno di viaggio il fedelissimo Antonio Medun, ch'esercitò lodevolmente un tal offizio presso alcuni miei precessori. Nel foglio che rassegno qui inserto alla Serenità vostra sonosi descritti li nomi di quegli altri individui che a norma della consuetudine debbono impiegarsi nelle restanti destinazioni.

Per secondar poi le paterne cure dell'eccellentissimo ser Zuanne Battista Da Riva, tendenti a perfezionare vieppiù l'educazione del di lui figlio ser Vincenzo, viaggia questi meco con molta mia compiacenza, dimostrando esso le migliori disposizioni per iniziarsi nella conoscenza delle pubbliche cose.

Finalmente, seguendo li principi di condiscendenza sempre praticati, mi accompagna eziando qualche nobile forestiere, e accorderò l'imbarco per il Levante a quattro religiosi che me lo chiesero quando, sull'esempio de' miei precessori, la Serenità vostra lo permetta.

Eccellentissimi signori, m'allontano dalla patria spinto dal vivo desiderio di potermi utilmente condurre nella delicatissima legazione di cui la sovrana autorità volle benignamente onorarmi, fattasi vieppiù ardua e malagevole in grazia delle circostanze de' presenti tempi, le quali non lasciano scorgere quasi nessun orizzonte che non minacci un avvenire torbido ed oscuro. La trepidazione però ch'io provo nel conoscer troppo fievoli le mie forze, in confronto all'importanza delle cose nella quali dovrò impegnarmi, viene in qualche modo alleviata dalla confidenza che ripongo nel clemente appoggio delle sapientissime istruzioni dell'eccellentissimo Senato, e facendo su di esse intenso studio, cercherò di corrispondere meno imperfettamente che mi sia possibile alle intenzioni della Serenità vostra. Con queste mire frattanto, venerando i recenti comandi di vostre Eccellenze relativi all'insolita circostanza che le navi son destinate ad entrare nel porto di Costantinopoli, procurai con ogni diligenza che il corredo delle medesime sia tale da sostenere possibilmente il pubblico decoro, proponendomi altresì la più vigile cura perciocché negli equipaggi di esse vi segui severa la disciplina, onde non arrivino que' motivi di moleste vertenze che in altri tempi diedero occasione di tenerle lontane da quella scala.

Dalla fregata Fama, li 22 aprile 1796. Acque di Fisolo. 


\section{2}

(Con 2 inserte)

Dopo di aver sciolto da codesti lidi a 19 del passato mese, fu sì lento il mio viaggio, contrastato ora dalla contrarietà de' venti, ora da calma quasi assoluta, che li susseguitarono, che soltanto il dì 5 corrente potei avvicinarmi al canale di Corfù, dove fui incontrato dall'eccellentissimo almirante delle navi nobil uomo Corner, che con somma vigilanza ed attenzione tesse le acque del Golfo sulla grossa fregata Gloria veneta la quale, e per il suo perfetto corredo e per la decenza e disciplina dell'equipaggio, manifesta abbastanza la particolare intelligenza ed attività del suo comandante e costretto dall'oscurità della notte e dal tempo minaccioso ad afferrare il sorgitore di Traù, nel giorno appresso mi posi di nuovo in cammino e dopo poche ore diedi fondo in questo porto, dove ho l'onore di segnare il presente ossequioso tributo del dover mio. In siffatto breve tragitto mi seguì sempre il prefato eccellentissimo almirante, prossimo ora a restituirsi alla sua crociera nel Golfo relativamente alle ricevute commissioni. Appena giunto fui visitato dagli eccellentissimi capitano e patrono delle navi e dagli altri nobil uomini delle due armate, grossa e sottile, a riserva però dell'eccellentissimo provveditor d'armata, il quale trovasi alla tutela del Golfo di Prevesa. Poco dopo venne a felicitare il mio arrivo il segretario dell'eccellentissimo provveditor generale Widmann, ed avendo io corrisposto colla missione del fedelissimo Alberti, si portò poscia egli stesso a vedermi. Oltre di aver questi pienamente soddisfatto a ciò che per metodo si conviene alla carica che ho l'onore di sostenere, con modo i più obbliganti e gentili volle pure esuberatamente praticar alla mia privata persona que' tratti di particolar cortesia che in ogni tempo si è compiaciuto di dimostrarsi. Adempite tutte le altre etichette dalle varie cariche, e dai corpi ecclesiastici latino e greco, militare e civico, rivolsi tosto le mie mire alle necessarie disposizioni per render possibilmente migliorato il materiale ed il formale della grossa fregata Fama, sopra la quale prese altresì imbarco il nobile di nave Grassi, che manifesta la maggior inclinazione per il pubblico marino servigio.

A questo fine cooperò con tutto l'impegno la primaria carica, la quale sempre attenta nel dar saggi di zelo e di diligenza, avea anche prima del mio arrivo, a senso delle ducali 2 dello scorso aprile, fatto allestire la leggera fregata Bellona per servirmi di conserva, avendo io destinata a comandarla l'attivo governator di nave ser $\mathrm{Nu}$ zio Querini terzo.

Come poi queste munizioni si trovavano mancanti di bandiere per fornire, a guisa della Fama, l'occorrente impaviglionamento alla suddetta fregata Bellona, e fattami conoscere da tutti questi intelligenti marini indispensabile siffatta decorazione eziando per la conserva, cercai di raccoglierne un adatto numero col minor possibile aggravio 
da privata persona, a tenore della acclusa nota, avendo perciò dovuto esborsare zecchini cento novantotto, locché mi lusingo verrà dalla Serenità vostra approvato. Volli però che le bandiere stesse fossero portate a debito del capitano della Fama, col dover di consegnarle in codesta eccellentissima casa dell'arsenale, al ritorno dell'eccellentissimo mio precessore, unitamente a quelle costì ricevute, onde farne uso in qualche altra occasione.

Accertar posso la Serenità vostra che, tanto per parte dell'eccellentissimo provveditor generale che per parte mia, nulla si è omesso perché in ogni modo possibile le navi siano ben corredate, per quanto lo permettono la deficienza di abili marinai e lo scarso numero di truppa di questo riparto. Anzi, a render vieppiù animato il mio zelo su questo interessante punto concorse una lettera dell'eccellentissimo mio precessore qui ricevuta, nella quale mi viene inculcata la maggior vigilanza acciò sia sostenuto in Costantinopoli il decoro delle venete insegne.

Nelle medesime mi fa cenno dell'opera che presta per concertare con il capitan passà i saluti e le altre formalità nell'ingresso ai Dardanelli, e nel caso probabile d'incontrarlo in Arcipelago, manifestando nello stesso tempo quanto importi di dirigersi in guisa di evitare ogni mala intelligenza e di assicurarsi di una perfetta reciprocità, locché richiede senza dubbio avvertenze maggiori oltre le volute da questa nuova combinazione.

Non posso passar sotto silenzio la singolare attività di questo eccellentissimo provveditore generale, che si applica con il maggior fervore e intenso zelo in tutti i delicati rapporti governativi di questa interessante provincia, ed essendo le benemerite di lui cure secondate dalli nobil uomini capi da mar, dal bailo e dalle altre cariche tutte nelle rispettive loro mansioni, questi popoli fruiscono i benefici effetti di un governo vigilante e giusto.

Privo delle necessarie nozioni sulla militare architettura, non sono in grado di render un adeguato conto a vostra Serenità di queste munizioni, caserme e fortificazioni, alcune delle quali sono in uno stato rovinoso, altre sembrano difettose e forse inopportune, ed altre mancanti affatto; ma però dalla vari dispacci del suddetto eccellentissimo provveditore general, e de' miei precessori, non meno che dalle relazioni di molti riputati uffiziali ingegneri, hanno vostre Eccellenze i più circostanziati dettagli di questo grave argomento, senza ch'io mi accinga a farne un rattristante ed imperfetto quadro.

Ben volentieri mi asterrei di far menzione anco della truppa, se l'aver più volte servito vostra Serenità nel dipartimento, che ha un'immediata relazione con le medesime, non mi avesse rese le idee più familiari in questo ramo che nel precedente. Non corrispondente l'esteso dettaglio alle forze, hanno da esso origine la mancanza d'istruzione, l'indisciplina e la soverchia fatica ne' soldati: male alloggiati questi ne' quartieri, invece del riposo vi trovano le malattie, ed il 
guasto delle loro uniformi e la mancanza di quegl'istituti che sono adottati dalla maggior parte delle nazioni rende la paga del soldato insufficiente a procurargli il necessario nudrimento, né vi è vigilanza bastante per garantirlo dalle voracità de' vivandieri. Trovandosi i militi in peggior situazione ancor quando sono imbarcati, si aggiunge alla necessità di prestar un duplice servigio il mortificante confronto di veder i marinai meglio trattati. La condizione degli uffiziali subalterni, atteso il giornaliero accrescimento delle vittuarie, merita pure d'interessar la pubblica provvidenza. Finalmente il metodo della reclutazione oltremarina per questa provincia non porge una quantità d'individui bastante a supplir alle naturali mancanze.

Sopra questi punti importantissimi non seppe il zelo mio trattenersi dal far qualche cenno, abbenché appieno conosca i rapporti ch'essi hanno con l'altro non meno riflessibile della pubblica economia, ma qualunque siasi l'effetto delle mie ingenue rappresentazioni ci fui condotto da un impulso di umanità verso tanti infelici e dal desiderio del miglior servigio di vostra Serenità.

L'ospitale militare merita di esser commendato. Mentre, dopo che l'eccellentissimo Senato vi destinò i padri di San Giovanni di Dio, sono in esso i soldati caritatevolmente assistiti e non presenta più come per lo innanzi, e come forse lo presentano tuttavia li ospitali subalterni dell'altre isole, l'orror del sepolcro anziché l'asilo della salute. Rimane nondimeno a desiderare che venga completato questo benefico istituto coll'edificazione della fabbrica provvidamente decretata dalla pubblica carità e dall'attenzione di questo provveditor generale, già incamminata con delle preliminari disposizioni.

Mi onoro di accompagnare a vostre Eccellenze la cauzione ritratta da questa pubblica ragioneria per le verificata consegna del denaro ed effetti preziosi destinati per Cantoni di Barberia, e ch'erano stati caricati sulla Fama per commissione dell'eccellentissimo Savio cassier e del Magistrato eccellentissimo de' V savi alla mercanzia.

Non ho lasciato per ultimo il dover mio di procurarsi, in ubbidienza alle ducali de 30 aprile decorso, tutti i possibili lumi sopra il molesto argomento delli due scogli Vuvala e Cara-Conzà situati nel golfo di Prevesa, ma le cose avvenute posteriormente alle accennate deliberazioni, e che saranno di già a cognizione dell'eccellentissimo Senato col mezzo de' dispacci dell'eccellentissimo mio precessore, e di questa primaria carica, provano bastantemente quanto malagevole siasi, nello stato in cui è ridotto l'affare, di ottenere una definizione conciliatoria i riguardi d'ambe le parti. Non può quindi il mio ossequio che limitarsi per ora ad una rispettosa riserva sino che, giunto a Costantinopoli, le istruzioni dell'eccellentissimo Foscari, i maggiori documenti che potrà forse in seguito avanzarmi questa primaria carica, dietro l'apposita conferenza tenuta colla medesima in cui si stabilì d'impiegare la destrità dell'eccellentissimo provveditor d'armata, che trovasi in quelle vicinanze, e le altre commissioni infine 
che la Serenità vostra riputasse d'impartirmi, mi pongano in grado di parlarne con più precisione.

Pronto essendo quasi per intero il corredo delle navi che debbano tradursi alla mia destinazione, mi lusingo di poter quanto prima dirigermi al Bosforo, desiderando solo che venti propizi rendano più sollecito il restante mio viaggio.

Corfù, li 11 giugno 1796

\section{3}

(Solo)

L'ossequiata ducale 2 del corrente giunse qui alle 12, in tempo che tutto era pronto sulle fregate Fama e Bellona per ripigliare il mio viaggio di Costantinopoli nel susseguente giorno. I gravi sensi delle medesime, che posero in somma angustia l'animo mio cittadino per le circostanze amare nelle quali sento trovarsi la patria, diressero la mia cieca obbedienza e mi prestai quindi con il maggior fervore e prontezza ad eseguirne i venerati cenni. Approfittando della lontananza di questo monsignor arcivescovo, feci trasportar il mio equipaggio nella di lui abitazione, scelta per dimora mia propria, e del numeroso seguito inseparabile da chi ha 'onor di essere destinato in bailo alla Porta ottomana.

Guidato dall'amor patrio e dall'ardente brama del miglior servigio, che il mio cuore preferirà sempre a qualunque riflesso e convenienza personale, feci con tanta sollecitudine verificare lo sbarco di tutto ciò che per i consueti regali e di mia proprietà trovansi caricato, che con mia soddisfazione li due suddetti legni formano parte della divisione che da questo porto prima si stava diretta diretta dall'eccellentissimo almirante.

Avrei fatto trasportare tutti gli effetti ch'erano sulla Fama a bordo della conserva mercantile, costì giusta il solito noleggiata, se fosse stata capace di contenerli, ma la sua portata non permise di aggiungere più niente a quanto aveva caricato la medesima in Venezia. Io la tratterrò qui col carico sino a che mi giungano le ulteriori determinazioni della Serenità vostra, prima delle quali non oso far cenno sopra quei spedienti che forse conciliar potrebbero il proseguimento del mio viaggio.

Questo eccellentissimo provveditor generale, animato da un zelo veramente patrio, impiega la maggio attività per eseguir con prontezza le pubbliche commissioni a fronte di molti ostacoli, e massime della deficienza di truppa, mercé le quali cure la divisione dell'illustrissimo almirante sarà fra breve susseguitata da un'altra diretta dall'illustrissimo patrone, come vostre Eccellenze ne saranno ragguagliate dalla medesima carica primaria. 
Per le successive disposizioni adunque non saranno per rimanere a questa parte che le galere, forse qualche fregata leggera, e la nave San Giorgio, che si sta acconciando in Guino sotto la vigilanza dell'illustrissimo capitano delle navi.

Nella incertezza del mio futuro destino, le critiche attuali combinazioni della patria e la profonda tristezza che me ne deriva per l'appassionato mio zelo per le pubbliche cose, mi fanno attendere con ansietà qualche più tranquillante riscontro accompagnato dai rispettabili sovrani comandi, che venererò sempre con la maggior rassegnazione.

Corfù, li 13 giugno 1796 secundum naturam

\section{4}

(Solo)

Si fa un dovere la mia obbedienza di render conto all'eccellentissimo Senato che soltanto nella sera del dì otto corrente giunse a questa parte il legno apportatore delle tre venerate ducali, segnate le prime del giorno due e la terza alli sedici luglio decorso.

I clementi sensi di quella, che si riferisce al pronto inoltramento da me fatto alle spiagge della patria de' legni destinati pel mio viaggio, dileguarono in qualche modo l'abbattimento del mio spirito cagionato dall'oscuro prospetto delle pubbliche circostanze e del trovarmi per così lungo tempo delle voci sovrane.

Incontrando pertanto la pubblica commissione mi farò a sottoporre ciò che reputerei conveniente per agevolare il sollecito mio tragitto a Costantinopoli, e sarà poi dalla sublime virtù pubblica il conoscere se conciliabile siano i riguardi della carica con le urgenti circostanze che lacerano l'animo mio cittadino. Ad oggetto di piantar sopra sode basi i miei riverenti pensieri, mi parve opportuno di passar prima d'intelligenza con questo eccellentissimo provveditor generale, dal quale rilevai che, zelante esso nel servire le pubbliche viste, ha già spedito nella corrente settimana a codesti lidi la fregata Brillante, che sarà susseguita in breve dalla Minerva e dalla Palma, e queste, con le altri navi già inoltrate, compongono tutta la forza che può per ora staccare da questo riparto. I legni poi che rimangono tuttavia consistono nella nave San Giorgio, coperta dall'illustrissimo capitano delle navi, la quale sortita da Guino sta ora completando il suo corredo; nella fregata Medea, che partita con la divisione dell'illustrissimo patrone dovette retrocedere, come sarà noto a vostre Eccellenze, per fiera burrasca sofferta, che gli fece perdere l'alboratura e la dilaniò in varie parti, dai quali danni si va riparando, e nella fregata Cerere, finalmente destinata come guarda porto al Zante. Altri legni minori ci sono pure, i quali non meritano di essere ricordati, 
perché inopportuni all'oggetto di cui si tratta, ma che per altro uniti alle galee riescono utilissimi ai riguardi della provincia.

Degli anzi detti tre legni, la Medea per la sua costruzione viene da tutti creduta la migliore per darmi imbarco e per far una decente comparsa. Se però l'Eccellenze vostre fossero per permetterlo, si potrebbe, affine di non impoverire maggiormente il presidio di questa piazza, minorare il consueto numero di soldati ed accrescer in proporzione quello di marinai per render completo il suo armo, come si pratica anche d'alcune straniere nazioni.

Una tale destinazione, che non verrebbe ad alterare quanto fu già disposto e verificato, sarebbe da sé sola bastante all'oggetto, ma nondimeno, qualora fosse giudicato conveniente di assegnar alla carica eziando una conserva, evvi la leggera fregata Cerere adattata all'uopo, la quale trovasi intieramente corredata ed equipaggiata. In questo caso dall'eccellentissimo provveditor generale sarebbe sostituibile interinalmente per guardia porto al Zante un legno di altra natura, e la Serenità vostra, se così lo credesse, potrebbe poi farne retroceder uno qualunque dei già spediti che si reputasse meno necessario ai riguardi della Dominante.

Non potendo la mia riverenza abbracciare il complesso delle circostanze che dirigono le sapienti determinazioni di vostre Eccellenze, né conoscere in tutta la sua estensione li vari complicati rapporti che indussero la sovrana prudenza a voler concentrare in prossimità a' propri estuari le sue marittime forze, sarebbe audacia il pronunziare sulla convenienza dell'indicato suggerimento. Per la qual cosa, ogni qual volta trovato non fosse conciliante tutte le virtù, si potrebbe forsi valersi di private imbarcazioni, su di che mi onorerò di riflettere che, segnatamente in questi ultimi tempi, non essendo permesso alle pubbliche navi l'ingresso nel canale di Costantinopoli, giunti i baili al Tenedo, salivano sopra uno dei legni di conserva, per non entrare nelle sdrucite galee turche che andavano a riceverli, e quindi si presentavano sovente alla capitale ottomana con bastimenti mercantili.

Coltivando pertanto siffatto pensiero rintracciai se in queste vicine scale vi fosse qualche legno atto a tale trasporto. Di fatti trovandosi qui il capitan Panà mi assicurò che alla Ceffalonia tiene vari bastimenti propri, e che inoltre attende fra poco un grosso vascello, col quale in altre occasioni servì alcuni miei precessori. A momenti pure sta per giungere altro adattato bastimento di ragione di certo capitan Ballarin, cosicché, piacendo a vostre Eccellenze questo secondo espediente, potrei forse lusingarmi, in grazia del confronto, di stabilire al caso un contratto a condizioni possibilmente discrete, o con l'uno o con l'altro dei suddetti due capitani.

Vero è che in tal modo non si farebbe uso del concertato ingresso delle pubbliche navi nel porto di Costantinopoli, ma non per questo ne sarebbe tolto alla Repubblica il dritto di occasioni nell'avvenire e se la Serenità vostra non ne approfitta in questo primo caso, egli è general- 
mente palese che le circostanze de' tempi conducono la prudenza de' governi a cercar di garantirsi in tutto le forme, tenendo raccolte le proprie forze e, siccome è nota la partenza dalla Dominante del ministro di vostre Eccellenze, così non è possibile di nascondere che anche il ritardato mio arrivo d'altro non può procedere che dall'accennata causa.

Cade poi anche in anoncio il riflesso che di necessità io debbo esser qui un inesperto cittadino, mentre quegli che regge presentemente la provincia, animato essendo in tutte le sue azioni dal miglior pubblico bene, è sempre intenso nel coltivare ne' sudditi l'affetto e la direzione che con non equivoci contrassegni manifestano per il loro principe, nell'amministrar la più retta giustizia e nel prestarsi con singolar fervore in ogni altro ramo che si riferisce al governo di questi popoli, della quale attività ne diede una luminosa prova nell'esecuzione dei recenti e pressantissimi sovrani comandi, avendo inoltre cercato, nello spoglio di milizie che dovette soffrire il presidio, di conciliar quei mezzi che, senza ricercare sovente peso all'erario, salvassero possibilmente i gelosi riguardi che merita in questa importante piazza.

Ma non basta che la mia stazione in questo luogo sia assolutamente inutile alle pubbliche viste, essa diviene ancora di aggravio all'erario perché prolunga la sussistenza di due baili, a meno che l'eccellentissimo Senato non credesse di maggior utilità al suo reale servigio il lasciar più lungo tempo in una difficile e gelosa legazione, qual è quella di Costantinopoli, un soggetto illustre per talenti e per consumata esperienza nel maneggio dei pubblici affari.

Sottoposti, in obbedienza alle sovrane commissioni, i mezzi che mi sembrano più facili per combinare con ogni altro riguardo il proseguimento del mio viaggio, e assoggettate insieme alcune rispettose riflessioni che concernono il soggiorno in quest'isola, desidero che i miei suggerimenti meritar possano la pubblica clemente approvazione, non già per sottrarmi dai gravi sagrifizi che soffre la mia privata economia, perché questi, singolarmente nelle calamitose circostanze presenti, esser debbono considerati per ultimo, e ogni buon cittadino deve farne con lieto animo olocausto alla patria, ma sibbene perché, mentre tutti trovansi occupati a cooperare alle gravi cure ch'esigono le urgenze pubbliche, io solo mi veggo astretto alla impossibilità d'impiegare, qualunque siansi i miei scarsi talenti, in servigio della Serenità vostra. Questo voto dettato dal più vivo amor patrio, e figlio di quei sentimenti che saranno sempre impressi nel mio cuore, non posso non manifestarlo all'eccellentissimo Senato, pronto per altro a venerar le sovrane sue deliberazioni, che soltanto imploro sollecite, affinché, qualora piacesse ch'io mi rimettessi in cammino, possa approfittare della favorevole stagione per ripigliar il viaggio.

Corfù, li 13 agosto 1796 secundum naturam 
Ordinata da vostra Serenità la retrocessione della grossa fregata Fama per il proseguimento del mio viaggio a Costantinopoli, l'arrivo delle ossequiate ducali 6 dello spirante agosto precedette di poco l'approdo in questo porto della fregata medesima, la quale diede fondo nella mattina del giorno 20 .

Impaziente di trovarmi alla mia destinazione, rimase assai sconfortato l'animo mio nel rilevare che li equipaggi si trovavano attaccati da perigliosa febbre epidemica manifestatasi in cammino in guisa che, toltine venticinque marinai sani, ma avviliti, e cinquanta soldati attivi, tutti gli altri, compreso il direttore delle milizie, erano o ammalati o in convalescenza, ed era poi in pericolo di vita il capitan Maticola, per cui restava la nave priva della principal marittima direzione.

Procuratomi pertanto le più precise informazioni, conobbi ad evidenza che non era possibile di prendere imbarco nella medesima senza porre a pericolo la mia persona, e tutti quelli del mio seguito, e senza perdere un tempo prezioso in grazia dell'avanzata stagione, onde fare i necessari espurghi ed eseguire eziando qualche lavoro occorrente nella parte materiale del pubblico legno.

$\mathrm{Mi}$ fu forza dunque di concertare una sostituzione con questo eccellentissimo provveditor generale che non esitò a suggerirmi la nave Medea, quella stessa che indicai come opportuna col mio riverente numero 4 , e che in breve sarà di tutto punto allestita, cosicché tra pochi giorni, spirando venti propizi, mi lusingo di mettermi alla vela.

Per non impoverire maggiormente di truppa questo presidio, oltre il natural equipaggio della nave e qualche rinforzo di marinai trovato necessario dall'eccellentissimo Provveditor generale, traendoli del corpo dei già esistenti, restrinsi la militar forza della nave stessa a due sole compagnie di oltramarini e ad un distaccamento di artiglieri.

Riflettendo poi il zelo mio alle pubbliche esigenze di marittime forze in questo geloso riparto, benché le sovrane deliberazioni 19 marzo e 2 aprile decorso abbiano trovato convegnente di assegnare alla reppresentanza di vostra Serenità una conserva, anche sostenere il decoro della Repubblica nel presentarsi dopo il corso di oltre un secolo alla capitale ottomana con legni armati, nella perplessità cagionatami dal silenzio delle ducali 6 cadente mi limito a fronte della consuetudine de' miei precessori, a far uso di una sola nave per il mio trasporto, tanto più che non giunse per anco a questa parte l'illustrissimo patrone colla sua divisione.

Non ostante per salvare in qualche modo la pubblica dignità, almeno nel mio passaggio per le suddite isole, questo Provveditor generale, dietro le ducali 5 dicembre 1795 che prescrivono di far frequentare i mari superiori e le acque dell'Arcipelago da uno o più legni, onde frenare i pirati che rendono mal sicura la navigazione mercan- 
tile ed espongono a frequenti lagnanze per parte della Porta, trovando necessario di destinare a scorrer quei mari, oltre la fregata Cerere, esistente al Zante, anche l'altra fregata Palma, questa coglierà il momento di mia partenza per trasferirsi all'adempimento delle già ricevute commissioni.

Ed in vero non potrei non ravvisare molto prudente ed opportuna ai riguardi pubblici siffatta destinazione, poscia che da recenti avvisi pervenuti si sa esservi nell'acque superiori alcuni corsari francesi, oltre qualche altro legno da guerra con bandiera inglese e due caravelle della flotta del capitan pascià ancorate in faccia alla Maina, e non sarebbe ancora improbabile d'incontrare in cammino lo stesso ottomano comandante, disceso nell'Arcipelago sino dallo scorso giugno, come l'eccellentissimo mio precessore ne prevenne questo provveditore generale, avendomi eziando la Serenità vostra con la ducale 10 luglio prescritta la maniera di contenermi quando ai concertati saluti nell'indicata circostanza.

Se nei precedenti miei numeri feci a questa primaria carica i meritati elogi per l'inattaccabil zelo con cui impegnasi nella reggenza di questa preziosa parte del Dominio di vostra Serenità, non posso ora dimostrarmi insensibile alle distinzioni praticatemi per tutto il corso della mia lunga stazione e non essere sommamente penetrato nel dover staccarmi da un cittadino che alle qualità esimie dell'uomo pubblico accoppia le più eminenti prerogative morali e che conciliatosi l'affetto dei sudditi lascerà in questa provincia traccie indelebili della sua pietà e beneficienza. Nel momento poi che ogni cosa è disposta per l'imminente mia partenza da quest'isola, non so nascondere a vostra Serenità la commozione che prova l'animo mio nell'allontanarmi sempre più dalla patria. Le ingrate circostanze della medesima per le incombenze dell'attual guerra in Italia, il politico generale prospetto dell'Europa, l'imagine di un oscuro avvenire, tutto contribuisce a rattristare il cittadino che con tanta clemenza pubblica fu onorato della difficile legazione di Costantinopoli. Sì, Principe serenissimo, stimolato dagl'impulsi di un cuor patriotico sento accrescersi il mio rammarico conoscendo che la tenuità delle mie forze economiche non mi permette di manifestare la patria in tutta la loro estensione i sentimenti dell'animo. Destinato a risiedere presso una delle principal corti, e a dover sostenere con un corrispondente lustro il decoro di vostra Serenità, userò nondimeno un rispettoso silenzio sopra i personali pesanti sagrifizi nel corso di presso che tre mesi in quest'isola, a fronte che le generose esposizioni delle ducali 2 luglio passato mi assicurino che sono presenti alle pubbliche $\mathrm{cu}$ re le mie convenienze, e sopporterò con cittadina rassegnazione il colpo fatale recato alle di già sconcertata mia economia, accertando insieme l'Eccellenze vostre che posi in opera le maggiori avvertenze perché confinato fosse nei più angusti limiti quanto cader doveva necessariamente a pubblico peso. Cercai quindi di usare il possibi- 
le risparmio nella straordinaria circostanza degli sbarchi ed imbarchi, de' trasporti ed alloggi per quella parte del mio seguito che non poté abitare nel palazzo vescovile e ne' quartieri; impiegai di concerto con l'eccellentissimo provveditor generale la più scrupolosa vigilanza, perché a pretesto di dover affrettar l'allestimento del pubblico legno non fosse abusato da chichessia nella somministrazione degli effetti indispensabili di queste munizioni. Procurai d'indurre il capitan della conserva mercantile, dietro il pretesto da esso fatto in precedenza, a restringer possibilmente le sue pretese di compenso per la lunga stalia in questo porto, giacché con la suddetta ducale 2 luglio m'ingiunge la Serenità vostra di trattenere a mia disposizione il solito legno da me noleggiato, riservandomi di assoggettare il preciso dopo il mio arrivo a Costantinopoli, e finalmente non mi permisi di ricercare da questa cassa generalato alcun suffragio oltre quello solito accordarsi a tutti i miei precessori. Se tenue si è l'olocausto, supplisca la magnanimità dell'eccellentissimo Senato accogliendo colla sua clemenza il riverente tributo d'un cittadino che conosce doveroso qualunque sacrifizio per conciliarsi vieppiù il pubblico compatimento.

Possa la fortuna essermi propizia in avvenire per non rendermene immeritevole anche il maneggio dei politici affari e nell'eseguire, giunto alla capitale ottomana, le prescrizioni della ducale 2 luglio decorso, che mi accompagna un articolo del mio precessore relativo alle comunicazioni fattegli dall'internunzio cesareo in proposito delle consuete visite di etichetta e essendo il predetto ministro non meno che l'inviato di Russia, per il loro carattere diplomatico inferiori di rango a quelli della Repubblica Francese, già riconosciuti da vostre Eccellenze, e della Batava, amica e alleata della prima, questi furono sempre preferiti nelle visite. Ma come gli altri due hanno protestato, perché i sovrani rispettivi non riconobbero per anche le prefate repubbliche, comparisce la mia tenuità malagevole l'evitare l'uno e l'altro scoglio, molto più che dall'articolo suddetto non apparisce esservi ora a quella corte il cambiamento di altro ministro di potenza neutrale, alla di cui condotta poter uniformarmi. Nella delicatezza della cosa, desidero che l'uso delle destre e prudenti avvertenze prescrittemi in siffatta congiuntura sia bastante ad allontanare qualunque amarezza, al qual fine non lascerò d'approfittare dell'esperienza e dei prudenti consigli del mio precessore. Che se poi credesse l'eccellentissimo Senato di confortare la mia insufficienza con quei maggiori lumi desiderevoli soltanto della sua sapienza, sarebbe necessario che mi fossero preventivamente note le pubbliche intenzioni.

Corfù, li 31 agosto 1796 secundum naturam 


\section{6}

(Con inserte 4)

Può finalmente l'ossequio mio prodursi a vostra Serenità da questa metropoli dove giunsi soltanto la mattina del giorno 11 corrente accolto nelle maniere più generose e nobili dall'illustre mio precessore.

Dietro a quanto significai col mio numero 5 intorno la partenza da Corfù, sciolto avendo le vele da detto porto nel giorno 7 settembre passato, fu bensì sollecito il mio viaggio fino al Zante, ma svanì ben presto la concepita lusinga di una navigazione costantemente propizia imperciocché soffiando tuttavia con forza i venti del nord dell'Arcipelago fui astretto a trattenermi 17 giorni nel porto di Milo, 5 nel canale di Tenedo e 21 all'imboccatura de' primi Dardanelli.

Innanzi di staccarmi dal porto di Milo rilasciai le correnti istruzioni alla leggera fregata la Palma, che mi seguiva di conserva acciò tosto che fossi rimesso alla vela, dietro le precedenti terminazioni della primaria carica da mar, potesse passare a Cerigo per iscaricare i pubblici effetti ch'erano imbarcati e congiungersi poi all'altra fregata Cerere, che dovea trovarsi nei passaggi superiori, per quanto rilevai nel mio passaggio al Zante. Non essendo io in allora autorizzato la vostra Serenità a trattenerla, perché tanto la ducale 29 agosto, che lo assente, quanto l'altra 6 ottobre, che approva d'essermi rimesso in viaggio con un solo legno le ho qui ricevute, fui anche condotto all'indicate risoluzioni dalli riscontri avuti precedentemente, che il capitan passà erasi qui restituito con la sua squadra e dalla fatta scoperta a 20 miglia in ponente dall'isola di Cerigo di una divisione inglese, composta di una nave e sei fregate, che incrociava quell'acque, per cui mi parve di decoro della Serenità vostra che in prossimità alle medesime fossero parimenti veduti de' pubblici legni.

Appena giunto ai primi castelli fui visitato dalli due comandanti dell'artiglieria, come lo fui al Tenedo dal gianizzer agà; mi praticarono in persona eziandio in persona degli atti offiziosi due chiaus ufficiali del capitan passà, e mi fecero complimentare per mezzo delli rispettivi segretari i due principi comandanti dell'una e dell'altra costa invitandomi oltre a alcuni presenti di bestiame e frutta, nelle quali occasioni, come in tutto il corso del viaggio, si prestò con molta diligenza ed abilità il fedelissimo dragomanno Antonio Medun. Siffatte intenzioni verso chi ha l'onor di rappresentare l'Eccellenza vostre mi determinarono a comprovare alli suddetti soggetti il mio gradimento con visibili contrassegni delle misure espresse nella inserta nota.

Venne in appresso al mio bordo il vice console dei Dardanelli e mi presentò le lettere dell'eccellentissimo mio precessore, che diligentemente m'istruiva riguardo i saluti da praticarsi, riportandosi ai più precisi dettagli che somministrati m’avrebbe vocalmente il detto vice console. 
Per i discorsi con esso tenuti, rilevai dunque che il capitan passà aveva ingiunto ai comandanti de' castelli di non salutare veruna estera bandiera quando questa non ne fosse la prima; che in seguito essendo state poste a campo delle preminenze sulla base di un preteso trattato, il Gran Signore in apposito firmano eccettuò da tal restrizione il nuovo ambasciatore della Repubblica Francese, nel supposto che potesse tenere la via del mare, ordinando che fosse salutato il primo, e che infine erasi inutilmente tentato d'indurre i detti comandanti a far lo stesso col ministro di vostra Serenità. Nella difficoltà dunque di conciliare sul momento i riguardi del pubblico decoro cogli ordini risoluti di tale proposito, sebbene l'eccellentissimo Senato si trovasse in ciò in parità di tutte le altre nazioni, fuorché della francese, conobbi che senza far nascere un mal inteso nel passaggio di un pubblico legno poteva esservi qualche spediente che lasciasse campo di meglio conoscere le cose in avvenire, tanto più che mentre mi trovavo ancora all'imboccatura del canale, essendo passata una divisione ottomana di quattro leggere fregate che discendeva a tessere le acque dell'Arcipelago, il comandante della medesima fece con somma prontezza precedere il saluto. Giacché dunque il comandante dei secondi Dardanelli, dove dunque si dovevano verificare i saluti mi aveva fatto sapere ch'era certo che dal canto mio non si sarebbe mancato ai soliti atti di cortesia costantemente praticati dai precessori, che anzi attesa la nuova combinazione di comparire con legno di guerra, si lusingava che li avrei con maggiori dimostrazioni sorpassate e ch'esso avrebbe cura che il mio passaggio non soffrisse ostacoli quando anche succedesse di notte, mi parve di dover prontamente aderire alla ricerca, restringendomi per altro alle solite misure. Avertato così di verificar il passaggio in qualunque momento approfittai di tal circostanza tentando di far regolar in modo la navigazione da passar i Dardanelli dopo il tramontar del sole, perché abbassandosi in allora la bandiera suolsi sul mare omettere il saluto. Se però la combinazione del vento secondò le mie brame, ebbi nondimeno a provocare la maggior angustia da ciò che sono per rassegnare a vostra Serenità.

Nel giorno 8 corrente avendo fatto mettere alla vela verso le ore tre pomeridiane con vento di ostro scirocco, fui colto da improvvisa calma per cui trasportata la nave dalla forza della corrente, fu partito di necessità di afferrare la costa d'Europa in un fondo di oltre quaranta passi. Poco dopo, soffiando di nuovo con maggior impeto lo stesso vento, sortì all'attività dell'intero equipaggio di ricuperare con l'argano parte della gomena sino ad alquanti passi lontano dal punto verticale all'ancora, nel quale momento cedendo essa alla forza del vento, che si faceva sempre più veemente, e trascinando la nave verso le purtroppo alpestri vicine rive, convenne decidersi al taglio della gomena, locché dietro i miei ordini venne immediatamente eseguito colla perdita dei generi risultanti dalla perizia dell'ammira- 
glio della carica, che inserta accompagno, onde sia sollevato il capitano dalla loro responsabilità. Superato in tal guisa l'imminente pericolo del naufragio, si diresse la prora col solo trinchetto e vela da straglio di maistra, che tosto rimase lacerata nell'interno del canale, ed oltrepassai i secondi Dardanelli a sera avanzata con lo stesso impetuoso vento; ma prima della mezza notte succeduta una violenta gruppata da ponente-maestro, e fattosi il mare burrascoso a grado che non era più possibile di scoprire la terra, a cagione della dirotta pioggia, convenne gettar l'ancora sotto la costa d'Asia dieci miglia superiormente alla Punta di Nogarà per ivi attendere il giorno, all'apparir del quale rasserenatosi il cielo, si poté proseguire il viaggio. Da tutto ciò scorgerà la Serenità vostra che se grazie alla divina Provvidenza mi ridussi colla pubblica nave a salvamento in questa capitale, non fui però assente dalla pena che cagionar suole la lunga navigazione e che dovetti io pure concepir la funesta idea d'un mar procelloso.

Non si tosto fu afferato in questo porto, che il capitan passà veduta la bandiera colla spada, rinovò i reclami altra volta rappresentati al precessore. Fatto a sé chiamare immediatamente il fedelissimo dragomanno Franchini e pretendendo che una potenza amica non dovesse portare uno stendardo ch'egli riguardava come un segnale di guerra, voleva che fosse levata dal medesimo la spada. Intesa tal rimostranza in mezzo alla confusione di quei primi momenti, ingiunsi all'abile dragomanno suddetto di riferire al capitan passà che siffatta bandiera era quella di tutti i pubblici legni per distinguerli dai navigli mercantili, i quali hanno la croce; che dal blasone che gl'inviava dei paviglioni di tutte le nazioni risultava questa verità, che qualche altra potenza, ed in particolare la casa d'Austria, portava il medesimo contrassegno, che non era in mia facoltà di atterare minimamente le pubbliche insegne e che conoscendo egli con quanta cura l'eccellentissimo Senato abbia sempre coltivata la buona amicizia ed intelligenza colla Sublime Porta, era certo che rimarrebbe convinto delle pacifiche intenzioni della Serenità vostra, alle quali risposte parve che si persuadesse.

Nello scendere dal bordo della Medea rilasciai le più precise commissioni, che inserte unisco, al magnifico ser Zamaria Bonlini, governador della medesima, onde allontanare i soggetti di pericoli e di male intelligenze, ingiungendogli di dipendere da quelle ulteriori che l'eccellentissimo precessore credesse impartirgli, giacché da quel momento rimaneva il pubblico legno sotto la sua immediata direzione.

L'attività, diligente attenzione e cittadino zelo del detto patrizio, qualità da me sperimentate nel corso del viaggio, mi assicuravano che sarebbe per corrispondere a sì delicato incarico.

Di fatti aggiungendosi alle di lui benemerite cure la cooperazione dell'abile sergente maggior Grisogano, direttore delle milizie, che tenne costantemente il presidio della nave nella più esatta discipli- 
na, proprietà di vestito e molto bene esercitato, come fece altresì l'attento capitano degli artiglieri Brasil con tutte le classi dell'equipaggio nel maneggio del cannone, e la vigilanza infine del sergente maggior Armeni capitano della nave stessa nel mantenere la polizia e nel far sì che i marinai siano disciplinati, destri e pronti nelle rispettive manovre, non che decenti ed uniformi nel vestito, posso assicurar vostra Serenità che quantunque la Medea conti un'epoca lontana di cantiere, la sua comparsa in questa metropoli fece concepire non svantaggiosa idea della regia veneta marina. Pochi giorni dopo aver posto piede a terra per mezzo di questo fedelissimo segretario, scortato dal dragomanno Ralli, feci partecipare il mio arrivo alla Porta, e ne fui corrisposto con il solito regalo di frutta e fiori, pregevoli in riflesso al primo visir che gl'invia. Susseguitò a questo la visita del dragomanno in abito di cerimonia, il quale con espressioni cortesissime mi manifestò i sentimenti rispettosi de' ministri della Porta verso vostre Eccellenze, ed i più obbliganti verso la mia persona, dimostrandosi molto soddisfatto di esser egli onorato di tal commissione. Terminata la cerimonia si congedò colle solite formalità, che furono da me accompagnate con le distribuzioni di metodo comprese nell'inserta nota, lusingandomi che la Serenità vostra sarà per approvarle.

Supplito aciò mi parve opportuno di dover praticare una particolare dimostrazione verso il capitan passà in grazia del passo fatto da questo ambasciator della Repubblica Francese Aubert du Bayet, al quale essendo nota la somma sua influenza sull'animo del sultano, e quanto ambiva d'essere coltivato, nello stesso giorno che mandò il proprio segretario a partecipare il suo arrivo al visir ed al reis effendi, gl'ingiunse pure di portarsi per il medesimo oggetto dal detto ministro, cosa non praticata per lo innanzi. Ma se conobbi tosto per l'una parte la necessità di lusingare la di lui ambizione e il pericolo d'indisporlo qualora non mi fossi uniformato al contegno del ministro francese, non volendo dall'altra introdurre direttamente una etichetta inusitata, lasciai scorrere alcuni giorni dopo l'indicata partecipazione alla Porta, e così il pretesto delli due fucili e quattro pistolle fatte appositamente fabbricare da vostra Eccellenze per fargliene un donativo, incaricando il fedelissimo segretario di presentargliele unitamente al dragomanno Franchini, e nello stesso tempo di felicitarlo in mio nome, nella quale occasione convenne fare altresì alcuni presenti al suo dragomanno ed uffiziali dipendenti, di cui come degli altri suespressi e indicati nelle note accluse ne imploro la sovrana sanzione.

Accolse egli il dono con i più pieni sentimenti, mostrò di pregiarne moltissimo la manifattura, si palesò in particolare soddisfatto del modo con cui glielo avea fatto giungere e non dissimulò nei discorsi tenuti la sua buona disposizione pel pubblico nome. E siccome parve desideroso di conoscere il comandante della pubblica nave, così d'intelligenza coll'eccellentissimo antecessore feci ch'andasse a vederlo in compagnia dei principali uffiziali di truppa e di marina. Gradì mol- 
to una tale attenzione e ne diede loro i più pieni contrassegni trattandoli con particolar cortesia; si trattenne in discorsi sulla costruzione delle venete navi e ordinò che si facesse veder loro tutto l'arsenale, nella qual circostanza occorsero poche mancie descritte nella suddetta nota. Lunedì scorso poi, senza far precedere alcun avviso, si portò a bordo della Medea per esaminarne dal vivo la costruzione, che riconobbe antica, ma rimase soddisfatto dell'alboratura, delle manovre e dell'agilità dei marinai trattenendosi lo spazio di oltre un'ora.

Benché improvvisa una tal visita fu trattato in quel modo che conveniva al di lui carattere, e mi corrispose con molte distinzioni verso il magnifico governatore e gli uffiziali. Al dragoman Franchini, che non mancò di portarsi a bordo tosto che il seppe, diede espresso incarico di complimentarmi e di assicurarmi ch'eragli noto quanto la Repubblica fosse sincera amica della Porta, come lo manifestavano gl'esempi delle passate neutralità, e che si compiaceva che avesse una florida ed attiva marina.

Da questo complesso parmi che si possa concepir la lusinga che il detto Capitan passà vada sempre più declinando dall'avversione che dimostrava in passato, in grazia delle benemerite cure dell'eccellentissimo mio precessore, e delle distinzioni usategli in questi pochi giorni del mio ministero. Ho altresì la compiacenza di rassegnar all'Eccellenze vostre che giusta li precedenti consigli del precessor medesimo e di quanto avea esso disposto precedentemente al mio arrivo, mi riuscì di dare tranquillo termine alle male intelligenze minacciate in questo proposito della preminenza nelle visite de' ministri stranieri.

Approfittando io dunque dell'esempio dell'ambasciator Liston d'Inghilterra, protestai a tutti i ministri stessi che vennero subito a vedermi, ch'era nemico d'ogni sorte d'etichetta, che mi lusingava che, coltivando la rispettiva buona intelligenza, gradirebbero un trattamento confidenziale ed amichevole, e che su queste basi mi sarei dato tutta la premura di visitarli. Di fatti mi portai tosto da essi in forma del tutto privata e ricevetti da ognuno le più piene dimostrazioni palesandomi in partenza la soddisfazione di abbandonare le antiche formalità, e quindi che il conforto di aver così combinati tutti i riguardi in circostanza tanto difficili come le presenti.

Rimangono ora e l'ingresso e le altre visite al sultano ed a ministri della Porta, che cercherò di verificare al più presto possibile, prendendo frattanto a tal uopo tutte le necessarie disposizioni.

Presente sempre all'animo mio cittadino le circostanze del pubblico erario e coerente a quanto mi feci un dovere di rassegnare col mio numero 5 da Corfù, quantunque dopo sei mesi di continuati dispendi siasi resa esausta la cassa del mio maneggio, indipendentemente da tutto ciò che la straordinaria combinazione fece cadere a mio privato peso, non mi credo permesso di trasmettere al rispettabile nome di vostra Serenità che la solita prima cambiale circoscritta a soli reali dodici mila e ottocento, che verrà presentata dal con- 
te Giuseppe Mangilli mio procuratore, supplicando dell'accettazione del pagamento alla scadenza.

Pera di Costantinopoli, li 25 novembre 1796

\section{7}

(Con inserte 4)

Rassegnato alla Serenità vostra ciò che riguarda il mio viaggio e arrivo a questa parte con separato dispaccio, non potendo nei primi momenti di questa legazione esser pienamente istrutto di tutti gli affari che la riguardano, e approfittando delle comunicazioni dell'eccellentissimo precessore, ho desiderato che congiunga alla mia la sua firma in questo ossequioso numero.

Ad accrescere l'amarezza in noi cagionata dalla continuazione delle molestie che derivano ai pubblici riguardi per le attuali circostanze, e che risultano dallo species facti trasmesso colla ducale 15 del passato ottobre, si aggiunse nei scorsi giorni l'infausta notizia della dichiarazione di guerra fatta dalla Reggenza d'Algeri alla Repubblica e pervenutaci colle qui unite lettere dei consoli di Algeri e Smirne e del nobil uomo governator di nave Orio, guarda rada al Zante.

Sebbene il detto console in Algeri avrà partecipato l'ingrato avvenimento all'eccellentissimo Magistrato dei V savi alla mercanzia, e da questi ne sarà derivata la necessaria comunicazione alla Serenità vostra, ciò non di meno riputiamo di non dover dispensarci dal far pochi cenni sulle cause che si vuole l'abbian prodotta e sulle previdenti disposizioni ch'era in poter della carica di prender per lume della mercantile marina.

Apparisce dunque dalla lettera del suddetto console in data 13 ottobre passato, che l'accennata dichiarazione abbia per origine l'istigazione di certo Sidi Muhamed, figlio del defunto dey Baba Aly, pretendendo esso che trovandosi colle sue genti a Smirne, ed avendole spedite in terra a far acqua, fossero queste assalite da 500 sudditi dalmatini e che ferissero anche diverse persone. Asserisce il console stesso che questo fatto non è che una mera calunnia, e tale pur la dichiara con sua lettera segnata 12 corrente l'altro console a Smirne, assicurando che i schiavoni non ebbero mai alcun contrasto cogli algerini, e che anzi nel soggiorno fatto a Smirne da detto Sidi Muhamed, gli fece egli molti doni e condiscese a varie sue ricerche, per cui protestò la maggior amicizia per la veneta nazione.

In sì critica circostanza però, conoscendo chi ha l'onore di servir vostre Eccellenze i gravi danni a cui potrebbero andar incontro i sudditi negozianti e capitani, fu ingiunto a tutti i consoli, con apposite circolari, di diffonderne generalmente la notizia, e di tener rag- 
guagliata la carica se sortissero pirati in corso, e quello poi di Smirne in particolare venne incaricato di procurarsi da quei comandanti ottomani dei documenti comprovanti l'insussistenza del fatto, onde poter smentire la calunnia.

A queste disposizioni preliminari susseguitò altra lettera del detto console segnata 14 corrente, che riferisce di non aver potuto ottenere da quell'agente algerino alcune attestazioni, che avea dapprima spontaneamente richieste, avendo anzi rilevato un nuovo motivo dell'ostile dichiarazione della Reggenza per cui pretendesi che i sudditi veneti, oltre le vie di fatto già accennate, abbiano eziando assediato il kan ove abitavano le genti di Sidi Muhamed. Aggiunse poi in essa lettera che per le deposizioni di un bastimento danese si seppe che in prossimità al Prodano due fregate algerine hanno predato il cotter veneto comandato dal capitano Viscovich, ed accompagna in fine supplica de' veneti capitani de' bastimenti che trovansi in quella rada, con cui implorano di poter esser convogliati da qualche pubblico legno in Corfù.

Nel rinovar quindi gli eccitamenti al console predetto per cercar di conseguire i contemplati documenti da quei comandanti ottomani, mentre non è da stupirsi se l'agente algerino non volle rilasciargli degli attestati contrari agl'interessi del suo padrone, si è pur trovato opportuno d'indicare al medesimo che tra otto o dieci giorni, dovendo di qua partire la nave Medea, saranno rilasciate opportune commissioni al magnifico governator della medesima perché abbia a ridursi nel porto di Cismè ed ivi, senza deviare dal suo cammino, debba prender sotto convoglio i bastimenti veneti che si trovano a quella parte per iscortarli sino a Corfù, al quale effetto si è anche scritto all'altro console in Salonicchio e se n'è anche diffusa la notizia a voce a tutti i capitani che sono in questo porto.

Rassegnate le prese disposizioni per la possibile tutela del commercio in una combinazione cotanto avversa, trovasi in dovere il ministro di vostra Serenità che ha l'onore di servire in questo difficile e delicato ministero, di far presente ancora tutto ciò che sopra questo interessante argomento può dar lume alle pubbliche deliberazioni, sebbene già a quest'ora la sovrana sapienza avrà preso le misure opportune per la condotta dell'affare.

Sia dunque palese alla pubblica maturità che il capitan passà, il quale ora manifestò delle buone disposizioni versi i veneti, ha un massimo legame con tutti i Cantoni, ed in particolare con quello d'Algeri, a cui fece dal sultano inviar in dono l'anno scorso due fregate, e qui tutti considerano ch'egli abbia sopra di loro una somma influenza e anche dai riscontri del console in Algeri è ciò riconosciuto, suggerendo egli come opportuno che i documenti che sortisse di ottenere in prova della falsità del frutto venissero convalidati con lettere dello stesso ministro, e lo dichiara pure la recente offerta che fece fare al veneto capitano Vianello di garantirlo con apposito firmano dalle molestie che gli algerini qualora volesse fare un carico di artiglierie per 
Tunisi, del che per non impegnare i pubblici riguardi cercò, dietro le insinuazioni della carica, di esimersi come da sé con privati pretesti.

Quantunque ci siano presenti le pubbliche massime dirette ad allontanar sempre la Porta ed i suoi ministri dall'ingerirsi nelle vertenze che insorgono tra la Repubblica ed e Cantoni di Barbaria, considerando non di meno che in mezzo ai mali cagionati alla patria dalle attual luttuose circostanze si è perduta con un colpo totale la sola risorsa che rimaneva, consistente nella sicurezza della veneta mercantile marina, la quale quasi sola fioriva a grado di eccitar l'altrui invidia, ci sembra di poter iudicare che il capitan passà destramente maneggiato potrebbe forse esser utile ai pubblici riguardi, giacché i principi politici dei governi dipendono sempre dalla varietà dei tempi e delle combinazioni.

In esecuzione delle venerate ducali 12 ottobre passato, giacché qui trovasi il capitano Mattia Palina, fu chiamato e severamente corretto dalla carica affinché sia più cauto nelle sue deposizioni avvenire, e sarebbe anche stato castigato con pena afflittiva se si fosse conosciuto esser derivato il fallo da malizia anziché da ignoranza ed inavvertenza.

Stessamente dietro i comandi dell'altra ossequiata ducale 18 del passato mese vennero praticate nei rispettivi offizi le annotazioni relative alla spedizione del dragomano ai Dardanelli in occupazione del futuro passaggio dei baili per portarsi a questa residenza.

Si è rilevato altresì con soddisfazione che in grazia delle attente sollecitudini del benemerito residente di Napoli siasi finalmente indotto il veneto capitano Giacomo Tiozzo a trasferirsi colla sua nave in questo porto per definire la già nota vertenza con questo capigì bassà Semptitin bey che non lascia però di meritar considerazione il protesto annotato dal capitano stesse nell'atto di rassegnarsi al comando, mentre prevedendo egli forse che la sua causa aver possa un esito sfavorevole sembra che maliziosamente e con cauto artifizio tenti di preparare delle ingiuste molestie al fisco.

Quando alla liberazione del turco fatto schiavo da un corsaro maltese, essendo stato deposto il reis effendi, che ne avea manifestata la premura, né sapendosi perciò con chi parlarne presentemente, resterà a lume della carica la lettera trasmessa da vostra Serenità al caso che da qualcheduno ne fosse promosso discorso.

Ragguagliata già la Serenità vostra della morte del tanto infesto pascià di Scutari, si trova in dover questa carica di rassegnare che avendo, col mezzo delle segnate persone che coltiva, estese le necessarie indagini per rilevare qual sarà il successore che gli verrà destinato, viene riferito che si disegna di dividere in tre differenti distretti quel passallaggio, ad uno dei quali sarà preposto certo Rustem passà, e che ai due altri saranno prescelti un nipote ed un parente del defunto Mahmud.

Pera di Costantinopoli, li 25 novembre 1796 


\section{8}

(Con inserte numero 3)

Nello scorso giovedì, approfittando anche della combinazione di trovarsi tuttavia in questo porto la pubblica nave Medea, verificai secondo il costume l'ingresso solenne che, ad onta della piovosa stagione, riuscì più pomposo e brillante dell'ordinario, avendo formato parte del corteggio gli uffiziali di truppa e di marina e un numero conveniente di soldati della nave medesima. Questa formalità costantemente praticata dai baili, e che ricorda degli antichi diritti della Repubblica, incontrò per altro delle difficoltà che potrebbono renderla in seguito contingente. Necessario di concertare col governo la giornata in cui dovea avere luogo, si portò a tal effetto il fedelissimo dragomanno Ralli dal reis effendi, il quale gli disse che rendevasi inutile siffatta cerimonia giacché essendo stato accordato alle pubbliche navi di entrare in questo porto, ingresso più solenne non ci poteva essere di quello di presentarsi in una metropoli con legni armati. Siccome nella trattativa di questo affare non si fece giammai dalla Porta su di ciò il menomo cenno, così rimase il Ralli sorpreso da un discorso cotanto improvviso e nuovo, cercando nondimeno con la sua destrità di persuaderlo. Inutile in quell'incontro ogni tentativo. Gl'ingiunsi di prodursi nuovamente al suddetto ministro e di fargli conoscere che la ricerca dell'ingresso delle navi ebbe in vista di facilitare l'arrivo del veneto ambasciatore in questa capitale, sostituendolo alle galere che venivano spedite al Tenedo per riceverlo; che anche i legni da guerra di varie altre nazioni avevano l'accesso per i castelli; che per annullare una consuetudine che conta una origine remotissima doveva necessariamente precedere una positiva convenzione fra i due sovrani; che con difficoltà la Repubblica avrebbe aderito a pregiudicare i propri diritti omettendo una formalità che distingueva il suo ambasciatore da quelli di tutte le altre nazioni e che qualora si volesse riguardarla come inutile quanto all'ingresso, tale pure si renderebbe riguardo alle consuete visite al visir ed al sultano, ed in tal basterebbe che le credenziali fossero fatte presentare dal bailo col mezzo del suo segretario. Benché vivamente apposte queste ragioni dall'attuale dragomano della Porta, che si va manifestando poco favorevole ai veneti interessi, si persuase alfine il reis effendi che per questa volta avesse luogo l'ingresso, soggiungendo che si dovesse partecipare al Senato l'intenzione del governo di toglierlo in avvenire, per lo che mi è d'uopo di renderne intese l'Eccellenze vostre, riflettendo per altro che ciò potrebbe forse aver avuto origine dall'impegno preso dalla Porta con questo ambasciator di Francia, il quale avendo chiesto di far un formale ingresso, come è già noto alla Serenità vostra, gli venne assolutamente negato.

I miei precessori in occasione della predetta solennità erano soliti di praticare un general invito nella sera a cui intervenivano anche i 
ministri stranieri. In vista però delle correnti circostanze ho creduto di dover astenermene, lasciando nondimeno aperta a chiunque la casa di vostra Serenità; ed infatti una tal condotta analoga alla direzione tenuta da principio per evitare l'etichetta, e con ciò qualunque amarezza, mi procurò la soddisfazione di vedere la sera stessa la parte più interessante del corpo diplomatico, avendo rimarcato soltanto uno studio particolare in alcuni ministri per non incontrarsi.

Con ossequioso mio numero 2 segnato da Corfù, indicai all'Eccellenze vostre la poca lusinga che avevo di veder terminare favorevolmente l'ingrato affare degli due scogli Vuvala e Caraconista nel Golfo dell'Arta, atteso lo stato in cui erano ridotte le cose. Si è purtroppo avverato il mio presagio, posciacché negli scorsi giorni chiamato il Ralli alla Porta, con molta insistenza gli fu chiesta una nuova lettera ostensibile di questa carica per ispedirla al passà di Jannina con la quale venisse eccitato l'eccellentissimo provveditor generale da mar alla pronta cessione delli prenominati due scogli. Quantunque munito di tutti i possibili lumi somministratimi dall'indefesso zelo del provveditor general medesimo, non essendo ad esso riuscito di ottenere alcun documento ottomano, mi manca la base principale per far dei nuovi tentativi onde sostener le pubbliche ragioni. Persuase l'Eccellenze vostre di questa verità, tanto più che la lettera da esso scritta al suddetto passà affronta di esser stata concepita con molta esattezza di raziocinio, non aveva prodotto alcun effetto, nello staccarmi da Corfù mi assicurò che avrebbe rinovati i suoi maneggi per conseguirne qualcuno nel proposito e sono condotto a credere che vani siano riusciti i di lui sforzi, mentre avendo ricevute recenti sue lettere relative alla dichiarazione di guerra della Reggenza di Algeri, niente mi ha egli trasmesso. Esauriti pertanto anche dalla destrità del mio precessore tutti i mezzi per l'esito propizio dell'affare e per tergiversarne la definizione, con difficoltà potrei per ora sottrarmi dal rilasciare l'indicata lettera, ma ad altra ricerca, per evitare il pericolo, che il passà di Jannina in vista del giudizio che ne fa il firmano e della proposta dilazione non passi alle vie di fatto, mi converrà accordarla, nel qual caso cercherò che l'espressioni siano le più innocue affinché il provveditor generale, da me pienamente informato di ogni cosa, possa regolarsi in modo d'allontanare qualunque disgusto. A questo passo di necessità sarò con dispiacenza condotto, avendo presenti le commissioni espresse nella ducale 8 ottobre decorso sul pensiero assoggettato da vostra Serenità dal zelo dell'eccellentissimo Foscari di proporre cioè il concambio delli due scogli in questione con il noto territorio di Butrintò riconosciuto di proprietà veneta, giacché non riuscì per anco di andarne al possesso. Ma l'insistenza della Porta sopra i detti scogli, non ammettendo più remora, verrebbe interpretata la proposizione come un pretesto per sottrarsi alla fatta ricerca e si esporrebbe di far nascere ciò che si vuole evitare. 
Riguardo poi all'ostile sopravvenienza di Algeri, per effetto della particolar destrità ed impegno dell'abile console in Smirne, mi pervenne un illam di quel mollà ed una lettera di quel voivoda, pur di Smirne, in cui si dimostra insussistente e falsa l'imputazione data alli schiavoni per aver un pretesto di dichiarare la guerra, come vostre Eccellenze lo rileveranno dalle autentiche e relative traduzioni che mi onoro di accompagnare a pubblico lume. Queste carte non agevoli a consegnarsi dai turchi, com'ebbesi a sperimentare nell'altro fatto succeduto parimenti a Smirne l'anno scorso, ed eziando per essere il detto voivoda agente dello stesso bey d'Algeri, provando l'aperta ingiustizia delle promesse ostilità, le avrei anche direttamente spedite a quel console acciò potesse farne uso, se dal poscritto in data 21 ottobre passato di una replicata del medesimo non mi risultasse ch'egli era astretto a dover partire sul momento da quella residenza. Vostra Serenità, a cui è presente il vero stato di questo molesto affare, conoscerà pur qual profitto sia sperabile di ritrarre dai prenominati documenti, le copie dei quali legalizzate in conveniente forma trattenni in questa segreteria per valermene a questa parte qualora in seguito l'eccellentissimo Senato lo trovasse opportuno.

Rilasciate già dall'eccellentissimo mio precessore le necessarie commissioni per la partenza della pubblica nave Medea, ed avendola essa fornita di tutti i mezzi occorrenti, non attende che il vento propizio per mettersi alla vela e per iscortar cammin facendo alcuni legni mercantili disposti di approfittare di tal occasione per esser convogliati, come fu reso noto a vostra Serenità. Si è cercato di dar imbarco sulla nave medesima ad alcuni schiavoni qui erranti, con che si combina la doppia vista di aumentare l'armo, che nelle attuali circostanze si rendeva necessario, e diminuire il numero dei sudditi vagabondi che recano continue molestie alla carica.

Con comune stupore si seppe giorni sono che la Porta aveva congedato alcuni uffiziali francesi ch'erano al suo servigio, quantunque compresi fra quelli protetti dall'ambasciator di loro nazione. Si vuole da taluni che i motivi addotti per tale licenziamento riguardino l'economia, ma d'altri pretendesi che ciò sia stato l'effetto dei recenti maneggi dell'inviato di Russia. Quest'ultima supposizione però sembra riprovata dal fatto, perché ve ne sono moltissimi al soldo del Gran Signore, e pure piuttosto ragionevole che un tal passo sia derivato o dalla loro incapacità nella protezione o dal loro carattere poco adatto ad indurre le truppe ottomane ad assoggettarsi alla disciplina europea.

In conseguenza degli uffizi di questo incaricato di Spagna sono stati rilasciati sulla loro parola due cavalieri di Malta ch'erano al bagno, fatti prigionieri dal capitan passà nella scorsa estate quando discese colla squadra in Arcipelago, e sopra i quali fece parola anche il mio precessore. Dopo aver ad essi fatti alcuni doni, volle che alla sua presenza si riponessero in petto le loro croci, nel che diede a divedere, che la compassione e l'umanità non sono prerogative al di lui animo. 
Le burrasche succedute ultimamente in Mar Nero, che fecero perire due legni mercantili russi, e per cui temesi di alcuni veneti legni che navigavano in quell'acque, obbligarono ad entrare nel Bosforo una fregata russa assai maltrattata, la quale diede fondo in faccia a Bujukdere. Questa circostanza avea generato dell'apprensione nel governo a grado che, tenutosi un consiglio segreto nella scorsa settimana, si sparse la voce che il detto avvenimento n'era fatto l'oggetto e di più che si fosse già recisa la testa al comandante dei castelli per averne permesso l'ingresso, locché non solo non si è avverato, ma anzi convien credere che la cosa siasi giudicata puramente accidentale perché si sa che vennero prestati al legno medesimo i necessari soccorsi per riparare i danni sofferti nella tempesta e per metterlo in istato di retrocedere.

Sono nella spiacevole necessità di rassegnare a vostre Eccellenze che apertesi all'occasione delle varie dispense solite farsi il giorno dell'ingresso i colli contenenti i generi avuti dal Magistrato alle rason vecchie, furono ritrovate sei pezze di saglia pregiudicate ad onta delle usate precauzioni per effetto dell'umidità contratta nel lungo viaggio e negl'imbarchi e sbarchi che si dovettero fare replicatamente. Essendovi sicurezza d'un rifiuto allor quando si avesse voluto far uso delle medesime, dovetti cercarne il cambio, che mi è riuscito di fare col veneto negoziante Marco Fua, mediante l'aggiunta di sole piastre 1200, delle quali imploro l'approvazione a cautela del mio maneggio.

Imploro parimenti il benigno assenso di vostra Serenità per il solito affitto della casa di campagna nella consueta somma di piastre 1000 , onde aver un sicuro asilo quando la peste fa stragi in questa capitale, avendo ottenuto a grande stento la casa stessa che abitarono altre volte i miei precessori.

Pera di Costantinopoli, li 10 dicembre 1796

\section{9}

(Primo)

Con somma mia sorpresa giorni sono il capitan passà fece a sé chiamare il dragoman Franchini ed in modi risoluti gli spiegò la di lui pretesa che i due bey delle galere non venissero defraudati dei consueti regali e solite mancie. Ad una tal inconveniente ed irragionevole domanda fecigli rispondere che siffatti doni si solevano dare ai bey perché soffrivano l'incomodo di portarsi colle galere al Tenedos ma che, cessatone in ora il bisogno per lo accordato ingresso alle pubbliche navi in questo porto, non avendo essi nulla fatto, non potevano pretendere veruna cosa. 
Donando esso però il maggior favore ai suddetti, non volle intendere ragione e mi fece ripetere ch'era certo che non lascerei di appagarli, mentre favorendo egli l'ingresso delle navi, col superar le difficoltà frapposte dal ministero, non avrebbe mai immaginato che da questo si volesse coglier motivo per recare pregiudizio alle persone che da lui dipendono. Palesatosi così stranamente fermo nelle sue pretese e riuscite inutili le replicate rimostranze a lui fatte dal suddetto esperto dragomano Franchini, fu in necessità alfine lo stesso di dirgli che sebbene non si avrebbe mai creduto ch'ei volesse rimunerati quelli che niente avean fatto, nondimeno, in riguardo all'interesse che sua Eccellenza manifestava, il bailo ne avrebbe ragguagliato l'eccellentissimo Senato per averne gli ordini relativi. Non si acquietò neppure a questa risposta, che venne da lui riguardata come un pretesto per cercar di deludere i desideri da lui spiegati, indicando che avea supposto ch'esser dovessero più facilmente incontrati. Le cure dell'eccellentissimo mio precessore e le direzioni da me tenute verso questo influente soggetto lo avevano reso meno contrario ai veneti interessi e però giudico necessario, se continui ad insistere, di non prolungar ulteriormente le contestazioni, onde non indisporlo di nuovo e mi appiglierò a qualunque espediente che concilii ne' modi possibili le di lui premure.

Cercherò prima di far qualche discreto presente agli accennati bey, non a titolo di diritto ma per altro, ma unicamente in riflesso alla protezione ch'essi godono del capitan passà ed in riguardo ai suoi uffizi. Se poi recredesse a questo tentativo, essendo pericoloso averlo contrario negli affari, mi lusingo che la Serenità vostra sarà per approvare se sarò costretto di contribuir ai detti comandanti delle galere li consueti regali e mancie.

Io provo sommo rammarico di dover esser strumento di pubblici impensati dispendi, ed è pur vero dire dolente la situazione di un ministro dell'eccellentissimo Senato poiché questo geverno ponendo forza a suo vantaggio la non buona posizione dei pubblici affari per le cose d'Italia, sopra i quali questi stranieri ministri cercano di accrescere la mala impressione con dei rapporti anche esagerati, esige irragionevolmente quelle che non dovrebbe e lascia bene spesso dubitare di non avere i riguardi corrispondenti all'amicizia che fra i due governi sussiste.

Infatti dopo aver la Porta spiegata la sua renitenza nel concedermi il solito ingresso, ho dovuto altresì veder insidiata la pubblica dignità rapporto alle visite d'etichetta che al gran visir ed al sultano si praticano.

Assicurato preventivamente che l'ambasciator di Francia non ricercava per ora le sue visite, giacché non erangli giunte le fregate che attendeva, incaricai il Ralli di chieder le mie, al quale venne anche fissata la giornata del 19 corrente per quella del visir, quando fu la stessa sospesa col pretesto ch'egli era in altri pressanti affari occupato. 
Mentre però stava maneggiando perché venisse un altro giorno sostituito, mi fu riferito, ed ho poi riscontrato col mezzo del dragomano stesso, che nel dì 22 era stata prefissata quella del ministro di Svezia Murajà. Siffatta improvvisa notizie, tenuta artifiziosamente celata, giacché al Ralli non fu mai fatto il più piccolo cenno né dal reis effendi né dal dragomano della Porta, e si seppe soltanto due giorni prima della sua verificazione, mi recò ad un tempo stesso somma dispiacenza e stupore.

Dispiacenza perché con una tale strana direzione si veniva ad offender il carattere d'ambasciatore, che posponevasi ad un ministro di terzo rango. Stupore poiché dopo un anno e più che questo ministro brigava per ottenerla, non avea mai potuto vederne segnato il momento, per le ragioni già indicate dall'eccellentissimo mio precessore in vari dispacci, e particolarmente per esser di nascita armeno e suddito del sultano.

Vedendomi nei primi momenti di questa legazione avvolto in un affare delicato per la pubblica dignità, i miei passi furono tosto diretti all'eccellentissimo mio precessore suddetto, onde col consiglio dello stesso e con quei riflessi che la sua sperienza avesse creduto di farmi, prender poi di concerto quelle determinazioni che fossero più adattate alla circostanza.

Maturandosi dunque da noi in tutti i suoi rapporti l'ingrato argomento, ad onta del nostro desiderio di allontanare contestazioni imbarazzanti e che potessero compromettere, abbiamo concordi riconosciuto che non eravamo in questo capo in arbitrio di dissimulare.

A ciò fummo determinati dai riflessi che la visita era stata non solo chiesta ma anco accordata; che il fu eccellentissimo bailo, poi serenissimo doge Renier nell'anno 1771 si credette in dover di sostener per pubblico decoro la preminenza di tali visite in un consimile caso contro l'inviato di Danimarca, come infatti vi riuscì colla soddisfazione dell'eccellentissimo Senato, e che la cosa si era già disseminata fra mezzo tutto questo corpo diplomatico.

Dietro a tali considerazioni si giudicò necessario il presentar delle rimostranze, e quanto alle forme ci attenemmo a farle giunger puramente vocali, poiché meno compromettenti, seguendo anche in questa parte il citato esempio. E siccome un tal argomento doveva interessare altresì gli altri ministri decorati d'un egual carattere, riputammo opportuno di far agli ambasciatori di Francia ed Olanda (il primo dei quali è anche nella detta circostanza di non aver per anco fatte le sue visite) le semplice narrazione dell'affare per rilevar qual senso avrebbe in essi destato.

Alle nostre comunicazioni, che accolsero con sentimento d'aggradimento, si manifestarono persuasi che fosse compromesso anche il loro decoro nella precedenza accordata al ministro svezzese, e spontanei offersero di far giungere contemporaneamente alle nostre le loro rappresentanze alla Porta. 
Si produsse pertanto il Ralli, e di nostra commissione significò che riusciva di molto rammarico al bailo di Venezia che dopo aver ottenuta la giornata per la visita si fosse quindi differita per accordar la preferenza ad un ministro di rango inferiore. Che altra volta nel 1771 in un simile tentativo fatto dall'inviato di Danimarca, la Porta, sebbene in allora non avea per anco prefissso la giornata al bailo, riconobbe il diritto superiore del veneto ministro e che, attesa la buona intelligenza che tra i governi sussiste, e che da tanti fatti per conto veneto fu in ogni incontro comprovata, confidava che la Porta avrebbe tenuta un'analoga condotta e cercherebbe un qualche spediente per far precedere nelle visite il bailo al Murajà, ovvero darebbe almeno quelle dichiarazioni che salvassero la dignità del carattere d'un ambasciatore.

A tali significazioni, appoggiate eziando da quelle dei dragomani di Francia e d'Olanda, rispose il reis effendi che non si avea mai supposto nell'accordar prima la visita al ministro svezzese di offender i riguardi dovuti all'amicizia della Repubblica ed al carattere del veneto ambasciatore; che soltanto era stato prima ammesso alla visita perch'era prima venuto e le avea il primo richieste; che la fissata giornata fu una sbaglio del teschierzi bassì, ossia maestro di cerimonie, e che così avea il sovrano stesso deciso.

Non potendo esser a noi soddisfacente una tale risposta, non restando più tempo ad alcun fruttuoso maneggio, poiché per la mattina susseguente dovea seguire, come si verificò, la visita del Murajà, abbiamo in quel giorno sospeso di far alcun altro tentativo.

Come però riconosciamo che all'offesa pubblica si converrebbe un qualche adattato risarcimento, così prima di far alcun'altra ricerca per ottener le visite, procureremo di conseguirlo, se sia possibile coll'uso di quei mezzi che riputeremo i più cauti e convenienti.

Se sorpresi che un artifizioso precedente silenzio i nostri sforzi non poterono aver in ora un esito felice, e se neppur riuscisse all'impegnato nostro zelo di averlo migliore nel destramente continuarli, confidiamo verso di noi clemente la sovrana bontà, che non vorrà attribuirlo a nostro difetto ma soltanto alla sfortuna dei momenti e delle combinazioni.

Pera di Costantinopoli, li 24 dicembre 1796

10

(Seconda. Con inserte numero 3)

Dopo l'ottenuto sovrano permesso espresso nelle ducali 2 luglio decorso di trattenere per la continuazione del mio viaggio sino a questa parte il noleggiato legno mercantile di consueta e sempre assentita conserva, e dietro le spiegate pretese del capitano sulla stalia 
nel porto di Corfù, dove per pubblico comando ho dovuto trattenermi, come ne ho già rassegnato riverente cenno nel mio dispaccio de' numeri 3, m'incombe in ora di render conto a vostra Serenità delle misure a cui si è ridotta questa pubblica spesa.

Il contratto di noleggio fissato in Venezia fu di reali 6000 sino a Costantinopoli, seguitando in conserva la pubblica nave coperta dalle insegne di questa carica. In tale contratto eravi la condizione che arrivato in questa capitale il capitan accordar dovesse giorni $10 \mathrm{di}$ stalia, ed altri 8 di controstalia, dopo il qual termine, non effettuato lo sbarco, fossi obbligato a risarcirli del tempo maggiore a reali 60 il giorno.

Colla base pertanto del contratto stesso, ricercò egli in primo luogo che avesse effetto la condizione suddetta per tutto quel tempo che trattenuto fosse nel porto di Corfù, e fino alla partenza per questa capitale ed in secondo luogo poi ch'esposto a perder per la detta stalia tutta l'estate, arrivando qui nella stagione d'inverno, né potendo promettersi nuovo noleggio che alla susseguente primavera, gli si dovesse perciò accordare doppio il suddetto noleggio, vale a dire dodicilmila reali in luogo dei sei mila del contratto.

Trovai bensì ragionevole la prima ricerca, mentre non dipartiva dal contratto che in quanto alla località della stalia, ma rispetto alla seconda protestai di non volervi assentire, giudicando indebita ed indesiderata la proposizione.

La mia fermezza nel rifiutarla e l'efficacia delle insinuazioni fattegli lo ridussero a rinunziarvi, contentandosi unicamente del compenso delli sessanta reali al giorno, li quali per tutto il tempo della fatta stalia in Corfù importano reali 4920, che uniti alli seimila del nolo sono reali 10920, delli quali ne imploro l'approvazione nei conti del mio maneggio.

Sono altresì nella necessità di richieder l'assenso di altri 1237 reali, spesa che dovetti immediatamente incontrare per conto di varie riparazioni nei più essenziali luoghi di servigio nel bailaggio detto vecchio, lo stato pericoloso dei quali non mi permise di frappor alcuna remora per un riattamento intanto provvisionale, ma non già solido, come ne sarebbe il bisogno.

Il giorno 15 corrente si è staccata da questo porto la grossa fregata la Medea per verificar le commissioni già accennate nel mio ossequioso numero 8. Al magnifico governator furono consegnati gli opportuni firmani, onde nel suo passaggio ai Dardanelli non sia per ritrovare ostacoli. Per ottenerli si avevano incontrate delle difficoltà, adducendosi che attesa la distanza del tempo in cui non entrano pubbliche navi in quest'acque mancavano li registri ch'indicassero quali n'erano i metodi. Infatti i primi che avevano rilasciati erano simili a quelli che si sogliono concedere ai bastimenti mercantili, per la qual cosa non essendo conveniente alla dignità dell'Eccellenze vostre, si restituirono chiedendone di nuovi nelle forme medesime che 
vengono dati a regii vascelli dell'altre potenze, ed infatti si ottennero in parità di quelli che furono recentemente somministrati ad alcune fregate spagnuole.

Nel momento che il sergente maggiore Grisogano, direttore delle milizie, e l'altro sergente maggiore Armeni, capitano del pubblico legno, si portarono formalmente a prender il congedo dalla mia carica, mi presentarono le qui unite due suppliche, ricercandomi d'inoltrarle al trono della Serenità vostra.

Tendono l'istanze del primo ad ottenere al militar presidio della nave la paga in buona valuta, appoggiando le sue ricerche alla convenienza, all'equità ed agli esempi sopra i quali milita a suo vantaggio la circostanza dell'ingresso del pubblico legno in questo porto, che l'obbligò ad una maggiore decenza nel vestiario ed alla necessità di dover acquistare dei viveri a carissimo prezzo in questa dominante.

A pari oggetto è diretta l'altra del secondo per gli uffiziali ed equipaggio di marina, per i quali parimenti si combina la particolar circostanza dell'ingresso, dell'altezza del prezzo dei viveri e le spese dovute farsi per un vestito decente ed adattato all'occasione.

Collo stesso incontro non posso per equità dispensarmi dal rassegnare all'umanità di vostre Eccellenze la supplica inserta prodottami dal benemerito sergente degli artiglieri Giovanni Damugliano. Dopo aver prestato un lungo e fruttuoso servigio, contestato d'autentici documenti e da pubblici favorevoli rescritti, egli è uno dei pochi nominati nel dispaccio numero 168 del fu cavalier e procuratore Emo che non abbia per anco ottenute le sovrane beneficenze. Desidera ch'io lo ricordi al trono di vostra Serenità, e contento com'io sono del zelo e dell'abilità con cui sostenne sulla nave Medea le ispezioni a lui proprie, mi presto a secondarlo e sarà poi della pubblica clemenza il prender sulle di lui istanze le deliberazioni che le sembreranno opportune.

Un veneto legno proveniente in tre giorni da Odessa recò a questa parte la notizia della morte dell'imperatrice di tutte le Russie, succeduta li 16 novembre decorso. Questo inviato ricevette le lettere di quel governatore che gliela parteciparono, ma attende ancora il corriere che deve spedirgli la corte. Nel serraglio si fecero al giunger della nuova tali allegrezze che neppure agli occhi delle persone meno accorte celar si poterono, forse confidando che colla vita di quella sovrana abbiano ad esser terminati i vantaggi dell'Impero russo.

Sono alfine arrivate ier l'altro le due fregate che da tanto tempo attendeva questo ambasciator della Repubblica Francese. Contengono esse una compagnia d'artiglieri, vari pezzi d’artiglieria leggera ed altri attrezzi militari, uniti a vari doni destinati per il sultano e suoi ministri.

Recatosene l'avviso da un dragoman francese al capitan passà, ebbe di sua mano con singolar distinzione il presente d'una ricca pelicia e di 500 piastre. 
Spiegatosi nel giorno d'ieri il gioco delle bandiere sulle dette fregate al momento del passaggio per il canale di questo sovrano, fu posta la bandiera ottomana nel luogo il più distinto ed onorifico; ma si rese osservabile che a fronte di essere in un porto neutrale si sieno avviliti i vessilli russo, inglese e portoghese coll'esporli in luogo ignominioso, mentre quello di Cesare non fu in alcuna maniera spiegato.

Pera di Costantinopoli, li 24 dicembre 1796

\section{1}

(Con inserte 4)

Sopra l'argomento delle visite per la preferenza accordata dalla Porta al ministro svedese ci facciamo un dover di render conto della condotta da noi tenuta in sequela di quanto fu rassegnato col numero 9. Perché appoggiata fosse dal fatto la dichiarazione giustificativa avanzata dal reis effendi al fedelissimo dragoman Ralli, che si concedevano le visite a chi primo era arrivato, si lasciò che seguisse l'udienza dell'ambasciator francese, benché affronte dell'arrivo delle sue fregate si fosse precedentemente espresso di non toglier la preferenza a chi ha l'onore di rappresentare la Serenità vostra. Approfittando poscia dell'opportunità dei scorsi giorni festivi e poiché nessun pressante affare lo esigeva, s'incaricò il Ralli di non prodursi alla Porta acciò senza ulteriori risentimenti trasparisse indirettamente un senso di dispiacenza. Difatti ci giunse a notizia che il dragomano della Porta, il reis efendi e il visir provano il maggior rincrescimento per l'avvenuto e che temendo che l'affare possa esser spinto più oltre desiderano che siano di nuovo richieste le visite. Anche lo stesso sultano disapprovò la condotta dei sopradetti ministri, come ci riferirono segrete persone, ed accreditate, alle quali la maestà sua accorda un libero accesso, sicché sperasi che in breve si potrà ottener la conveniente riparazione alla pubblica dignità.

Con nostro sconforto poi ebbe un termine sfavorevole l'altro affare dei regali ai bey delle due galere. Il capitan passà, com'erasi preveduto, avendo rinovato le sue domande con più forza di prima, convenne, per non indisporlo, aderirvi ed attenersi alle misure usate per lo adietro dai precessori, come risulta dall'inserta nota.

Questo ambasciator di Francia con l'unita memoria espone in sensi acerbi come la pubblica nave Medea, che indica fosse ancorata a Santo Stefano, nel passaggio delle fregate di sua nazione, abbia alla vista delle stesse ammainata la propria bandiera. Non potendosi da noi ritrar le necessarie informazioni nel proposito, si è creduto di rispondergli nel modo che l'Eccellenze vostre dall'annessa copia rileveranno. Avendo poscia avuto l'incontro di naturalmente vederlo, 
si cercò di renderlo persuaso che l'accaduto può esser derivato da tutt'altro che da mancanza ai riguardi dovuti per l'amicizia che tra i due governi sussiste. Sperasi in tal modo di aver diminuita l'amara sensazione cagionatagli dall'avvenimento e che i di lui rapporti al Direttorio esecutivo giungeranno, per quanto promise, con minor risentimento di quello si poteva temere. A ragione ci lunsinghiamo altresì che le nostre dichiarazioni lo abbiano alquanto temperato, mentre nel seguito impaviglionamento in giorno solenne delle dette fregate videsi esposto in luogo d'onor il vessillo di San Marco, che per lo innanzi non era stato mai spiegato. Acciocché poi alla pubblica autorità non siano ritardati possibilmente gli occorrenti lumi al caso d'un reclamo, ci comparve convenevole di render avvertito dell'emergente il Provveditor generale da mar, onde si procuri tutte le necessarie informazioni, dalle quali speriamo che abbia a risultar provata l'innocenza del nobiluomo governator, e di eccitarlo allo stesso tempo a farne giunger solleciti riscontri a vostre Eccellenze.

Già prossimo a spirare il quinquennio dalle leggi stabilito per l'esercizio del carico del console di Salonicco Giuseppe Coch, in vista delle circostanze di sua numerosa famiglia, ho creduto io Vendramin di devenire alla di lui conferma e ne accompagno a vostra Serenità la relativa terminazione, affinché sia dalla sovrana autorità approvata.

Pera di Costantinopoli, li 10 gennaro 1797 more veneto

\section{2}

(Senza inserte)

Le calamitose attuali circostanze accrescono di giorno in giorno l'angustia che prova l'animo mio cittadino nell'esercizio del ministero che la sovrana autorità si compiacque di conferirmi. Dopo la fata dichiarazione di guerra della Reggenza di Algeri tutti i veneti capitani che qui e nelle vicine scale si trovano, sono condannati a starsene oziosi in porto con gravissimo loro danno. Quelli qui esistenti circondano continuamente la carica, si querelano, producono delle incommode ricerche e sono immersi nell'abbattimento e nel dolore. Non lascio d'invigilare perché coll'ottenimento di particolari firmani e con altri maneggi non vengano compromessi, come alcuni lo avevano di già tentato, i pubblici delicati riguardi. Cerco altresì di animarli facendo ad essi sperare forse non lontano il termine della loro triste situazione e di allontanarli possibilmente dall'idea di vender i loro bastimenti. Confesso nondimeno ch'è sommamente sconfortante per il mio zelo patrio il vedermi privo di siffatti momenti dell'autorevole suffragio delle pubbliche sapienti istruzioni, scorta indispensabile per non render ad ogni passo incerta e dubbia la mia direzione. 
Di non minor rammarico mi riesce il mancar di sicuri rapporti sui fatti che accadono in Italia tra le armate belligeranti, imperciocché alle voci sempre sfavorevoli ai pubblici riguardi che or dall'uno or dall'altro partito si vanno spargendo secondo le particolari loro viste, non posso opponere che il silenzio ed una non sempre opportuna dissimulazione. E se anche talvolta questa condotta mi libera dalle maligne riflessioni dei stranieri ministri, non so poi come regolarmi verso la Porta che fa continue ricerche ai dragomani di vostra Serenità su quanto succede nello Stato Veneto e ne domanda schiarimenti, per la qual cosa oso implorare la continuazione dei soliti species facti di cui sono mancante dopo la ducale 15 ottobre decorso.

È osservabile la celerità con cui procedono i lavori in questo arsenale con l'assidua soprintendenza dello stesso capitan passà, il quale perciò è pressoché invisibile. Essendomi quindi proposto di fargli una privata visita non credetti fin ora opportuno di verificarla, molto più che mi si assicura riuscire al medesimo disgustoso tutto ciò che lo distrae dalle sue occupazioni per le quali è al sommo grande appassionato.

Sopra il bacino che si è qui proposto di formare insorsero gravi questioni fra gl'ingegneri francesi ed uno svedese sulla maniera più facile e meno dispendiosa di costruirlo. Alla fine quest'ultimo ebbe la preferenza, in grazia anche delle sperienze praticate con alcune trombe da esso fatte, per mezzo delle quali gli riuscì di vuotare in breve tempo un vasto recipiente di acqua.

Per l'altro mentre l'ambasciator di Francia trovasi a bordo della fregata la Diana, di sua nazione, vi si portò il capitan passà, che fu salutato in modo particolare con molti tiri di cannone. Poco dopo d'esserne partito dimostrò il suo gradimento al ministro inviandogli in dono un ganzar, due pistolle e un trombone di ricco e bel lavoro ad uso turco.

Non ordinaria si fu la pompa colla quale il suddetto ambasciatore verificò la sua visita al primo visir. Era egli vestito coll'uniforme da generale, circondato dai vari uffiziali che ha seco, preceduto dagli equipaggi militari e marini e dalla compagnia di cannonieri leggeri, che si dice che possa rimanere al servigio della Porta, tutte con armi e tamburo battente, chiudendo poi il corteggio tutti i nazionali francesi qui dimoranti. Oggi dovea seguir l'altra visita al sultano, ma venne differita, e da taluni se ne adduce per motivo la maniera straordinaria colla quale intendeva praticarla. Dicesi che oltre la suddetta compagnia di cannonieri, ora provveduta anche di cavalli, e tutti i soldati armati che avea seco quando andò dal visir, volesse farsi preceder altresì dai pezzi d'artiglieria leggera, che in nome della Repubblica Francese deve presentare in dono al Gran Signore e aggiungesi ancora che prevedeva di non attender il visir, com'è di consueto, ma che invece si trovasse questi a riceverlo, e non volea finalmente esser introdotto alla presenza del monarca dalli due capigì bassì, 
che danno braccio alli stranieri ministri e li conducono in faccia al soglio. Se questi siano i veri motivi del differimento io non posso con certezza asserirlo, e qualor siano tali sembra naturale che la Porta, attentissima ai metodi antichi, e volendo aver riguardo alla Russia, allo straordinario ambasciator della quale non accordò che piccole distinzioni, provi molto imbarazzo a secondarlo.

Corre voce che la mala intelligenza che sussisteva tra la Repubblica Francese e la corte si Svezia per il noto matrimonio con una principessa di Russia, sia finalmente cessato e che siasi ristabilita la ministeriale corrispondenza tra queste potenze, cosa che qualor sia vera non la ignorerà l'eccellentissimo Senato per i riscontri che dalla diligenza del nobile a Parigi gli saranno stati avanzati, e ciò che può accreditare siffatta notizia si è che siccome questo ministro svedese all'arrivo dell'ambasciator Du Bayet si era portato a visitarlo, ed egli non lo avea corrisposto, così ieri il suddetto ambasciator gli restituì formalmente la visita.

È qui giunto un inviato di Persia spedito da Agà Muhamed kan con un seguito di circa trenta persone, riputato generalmente soggetto pieno di talenti e di sperienza avendo sostenuto varie ambascerie, e fra le altre presso l'imperator del Mogol. Credono taluni che la sua missione abbia per iscopo d'implorare il soccorso della Porta contro la Russia, altri lo vogliono incaricato di ricercar la sua mediazione per ottener la pace.

Nella mortificante combinazione di dover servire l'eccellentissimo Senato in un ministero che di natura sua pesar deve purtroppo sull'erario, quantunque il mio zelo procuri di uniformarsi con cittadina moderazione alle critiche circostanze de' tempi, non posso però dispensarmi dall'implorare i necessari suffragi. Sono note a vostre Eccellenze le vicende del mio viaggio, la mia lunga dimora a Corfù con tutto il seguito che porta seco la carica, i replicati imbarchi, sbarchi e trasporti di robe e persone, le sovvenzioni date agli equipaggi di truppa e di marina e l'esborso fatto per il noleggio e stalia del concessomi solito privato bastimento, che dovette ascendere alla somma di già rassegnata. A tutto questo debbonsi aggiungere le panatiche e salari somministrati a quelli che hanno titolo legale per esigerli, alcuni indispensabili restauri verificati in vari luoghi di questa casa di vostra Serenità, che minacciavano rovina, e gli esborsi primordiali metodici nella istallazione di questa carica, oltre l'ordinarie dispense dei pubblici effetti, senza osar di far alcun cenno sull'afflitto mio privato economico che per le fatali circostanze presenti, soggiacque a gravissimi pesi non risentiti certamente da verun de' miei precessori.

Tali sono le fonti passive che angustiano la casa bailaggia nella incessante progressione degli aggravi che le incombono, non suffragate dopo il mio lontano distacco dalla Dominante che dalle somme levate nelle consuete misure dalle camere di Corfù e del Zante, e dalla pri- 
ma cambiale di metodo già spedita e per tutti questi motivi pertanto supplico la Serenità vostra dell'accettazione e pagamento in scadenza della cambiale straordinaria di reali quindici mila che inoltro al mio procuratore conte Giuseppe Mangili per altrettanti dal medesimo rimessimi, affine di essere in grado di far fronte agli imminenti pesi che mi sovrastano a quest'ambasciata.

Pera di Costantinopoli, li 10 gennaro 1797 more veneto

\section{3}

(Con inserte 4)

Le nostre lusinghe che un adattato compenso salvar dovesse i riguardi della pubblica dignità compromessa nelle visite in precedenza accordate all'inviato di Svezia furono da un esito felice secondate, e con vera compiacenza umiliamo alla maestà dell'eccellentissimo Senato una lettera di dichiarazione e di giustificazione scritta dal dragomano stesso della Porta.

Questo era appunto quello che da noi si attendeva. Dalle sole vocali espressioni non potevamo salvar interamente il decoro del carattere del ministro di vostre Eccellenze e si esigeva uno scritto che le comprovasse e che ponesse un freno alle altrui maligne riflessioni. Veniva riputata difficile la cosa, poiché rare volte, e soltanto nei casi i più interessanti, dai ministri della Porta si scrive; e questo appunto accresce il nostro contento di poterci produrre con un tale risultato nella circostanza presente.

Avessimo ottenuta qualche giorno prima la lettera, in grazia dei maneggi fattisi dalle segrete persone da noi impiegate, ma questo signor ambasciator della Repubblica Francese, che volle assumersi il carico di mediatore e rendersi all'una e all'altra delle parti accetto, ne ritardò necessariamente la comparsa giacché dovette passar per le di lei mani nel desiderio ch'ebbe d'accompagnarcela con sua memoria.

Alle due carte suindicate abbiamo formato le risposte, che umiliamo, e se abbiamo admessa le fatteci giustificazioni, fu da noi però giudicato conveniente, onde far vedere com'erano ben fondate le nostre prime rappresentazioni, di richiamar in iscritto sott'occhio l'esempio dell'anno 1771, e di rimarcar che il sistema preso dalla Porta che primi debbano esser introdotti all'udienza i primi arrivati era per certo a quell'epoca posteriore.

Noi già non fummo né i soli né i primi ad esperimentar in ora l'effetto del suindicato metodo dal ministro ottomano stabilito, e direm così proclamato. Avendo preceduto nelle visite il ministro francese vi si adattò egli il primo in confronto dell'inviato di Svezia, e le dichiarazioni poi fatte che soltanto per equivoco era stata prima concessa 
la giornata al ministro di vostre Eccellenze ci fece esser pienamente anche in quel rapporto tranquilli.

Il suddetto ambasciator verificò nei giorni scorsi la sua visita anche a questo monarca. Non la eseguì però nel modo che avea disegnato di fare poiché non vi furono i soldati delle fregate, che comparvere nell'udienza avuta dal visir a tamburo battente, e colle baionette in canna, e la compagnia di cannonieri si presentò al serraglio e passò in Costantinopoli senza ostentar le armi, che soltanto nude si videro dopo il tragetto del canale nella marcia fatte nel borgo di Pera.

Anche nelle ricerche avanzate, onde nella cerimonia da lui eseguita nulla vi fosse di ciò ch'ei considera atto di disprezzo e di superiorità di questo governo sulle franche nazioni, non ebbe motivo di rimaner pienamente contento e fu, è vero, introdotto in un sito più comodo di quello che si accostuma per ivi attender d'esser ammesso alla presenza del sultano; non diede nel cammino la dritta al chiaus bassì; non fu preso per le braccia dai capigì bassì nell'atto di prodursi all'imperatore, ma la stanza non fu quale se l'attendeva, il chiaus bassì non potendo aver la dritta non volle accompagnarlo per istrada e nella sala dell'udienza fu circondato da numero sì grande dei suddetti capigì bassì che non poterono avervi ingresso che soli cinque dei suoi. Non riuscì poscia minimamente nel disegno che avea di non esser obbligato ad attendere il visir, il quale anzi si fece qualche tempo aspettare, sicché essendogli andato fallace questo punto essenziale, non si considerano da questi stranieri ministri tali le fatte innovazioni che meritar potessero che si entrasse per esse in maneggio.

Ei pure confessa di non aver avuto nella suindicata parte il suo intento, fu sensibile oltre modo al ritardo che gli si fece soffrire e si dice che abbia prodotto delle forti rimostranze alla Porta. Ma nonostante non lascia di considerar pregevoli le cose già ottenute, accenna che non si adoperò per aver egli solo delle particolari distinzioni, ma che fu suo pensiero di sostener col suo decoro anche degli ambasciatori delle altre nazioni, ed eccita me Vendramin a procurar di conseguirle giacché lo debbo immediatamente susseguitare.

Si cercò di esimersi destramente da un tal impegno, e vi fummo indotti dalle seguenti riflessioni.

È discorde l'opinione sulla importanza dei cangiamenti fatti per conto del ministro francese: taluno crede che non essendo stato accompagnato dal chiaus bassì abbia piuttosto avuto uno svantaggio; non si sa come saranno per dirigersi i nuovi ambasciatori delle altre nazioni; se qui giunga prima di farsi la pace un ambasciatore dell'imperator di Russia si farà forse un vanto di sprezzarli e di attenersi al metodo antico appunto perché fu variato dal ministro francese. E poi nella circostanza in cui ci troviamo, e che fu esposta col precedente numero 8, che si voglia (forse per l'altrui maligne insinuazioni) toglier a chi gode l'onore di rappresentar la Serenità vostra le veramente singolari distinzioni di aver un solo ingresso solenne, d'esser accompa- 
gnato fino alle stanze superiori dal chiaus bassì e di dar un pranzo nel proprio palazzo ai gianizzeri, cosa che soltanto è fatta da questo sultano, non ci sembrò prudente il mostrarsi desiderosi di alterar il vecchio cerimoniale rapporto alle udienze, per non fornir un appoggio di alterarlo anche nell'altre parti a norma delle ingiuste spiegate intenzioni.

Perciò noi crediamo di doverci tenere in un perfetto silenzio e di operar attenti soltanto le direzioni della Porta, la quale se spontanea procedesse poscia a qualche innovazione ch'ella dichiarasse di voler osservare verso tutti gli ambasciatori stranieri, non avrebbe perciò qual si sia diritto di sostenere le mal fondate pretese.

Intanto nella visita da me Vendramin eseguita al visir non vi fu alcun cangiamento, come niuno ve n'ebbe per l'ambasciatore di Francia, giacché furono posteriori i suoi reclami e ebbi secondo il solito al mio fianco il chiaus bassì, che non volle, come abbiam detto, intervenire all'accompagnamento dell'ambasciator suddetto nell'udienza dal sultano ottenuta. Si può da questo dedurre ch'egli sia per intervenir anche in quella giacché se pensasse di non dovervi assistere, perché da me si sostenesse la pretesa di non tenerlo alla mia dritta, non si sarebbe veduto neppur nella mia prima comparsa.

Vedremo quello che sarà in seguito per arrivare, ma frattanto abbiamo la soddisfazione di umiliare che l'accoglimento il più grazioso fu fatto al ministro di vostre Eccellenze, che con singular distinzione fu ammesso all'udienza senza che attender lo si facesse un solo momento, e che i sentimenti della maggior considerazione per l'augusta nostra patria e della maggior gentilezza verso chi ha l'onor di rappresentarla furono dal primo ministro di questo impero professati.

Speriamo approvate dalla sovrana clemenza dell'eccellentissimo Senato le suindicate nostre direzioni, nella quali abbiam avuto per oggetto di salvar tutti i pubblici delicati riguardi e di non infirmar le ragioni a cui sono appoggiate le distinte prerogative di questa rappresentanza.

Pera di Costantinopoli, li 25 gennaro 1796 more veneto

\section{4}

(Con una inserta)

Per darmi una norma della mia condotta e per sollevarmi in parte almeno dalla imbarazzante situazione in cui mi trovo, opportune ed attese mi pervennero le ossequiate ducali di vostra Serenità 17 dicembre decorso.

Venerai in esse le sovrane determinazioni nella risposta data al dey di Tripoli il cui ambasciatore, qui giunto da tre giorni, incalzava le sue ricerche, riputava un pretesto le mie asserzioni di non aver avuto alcun 
ordine e mi costituiva in una responsabilità di grave peso al mio cuore. Ricevuta avea appena la lettera di quel veneto vice console, che m'indicava la prossima partenza del detto ambasciatore, e la somministrazione anticipata ch'ei sarebbe per chiedermi delle venture annualità, veggo accennarmisi da Smirne ch'egli era giunto in quelle vicinanze e presentarmisi le lettere di credenza che dovea recarmi di persona.

M'avvidi dell'oggetto per cui con tanta impazienza m'avea inoltrata la lettera, perché cioè tenessi pronti al suo arrivo i dinari, e non m'ingannai nella supposizione perché entrato appena in questo porto fece scender primo un suo confidente che si diresse alla casa di vostra Serenità per ricercarmeli.

I riflessi fattigli appoggiati dalla verità e dalle circostanze che mi mancano le istruzioni, ch'io non potevo prendermi alcun arbitrio, che la somma era gonfiata, che non vi è alcun asempio di tale dimanda, che sorpassava del triplo la convenuta annualità e che la casa da me amministrata avea soltanto il dinaro necessario alle natural sue esigenze, furono di niun peso presso il suddetto confidente.

Rispose che si attendeva dinari invece di parole, che non era d'amico la mia negativa e che riporterebbe il tutto al suo principale.

Volendo sperare che forse dal carattere di costui dipendesse l'aspra risposta, e non volendo appoggiare a un infido relatore la forza delle mie ragioni, feci, coll'apparenza d'adempir un atto offizioso, andar tosto il Franchini dall'ambasciatore. Fu accolto superbamente, lo si lasciò appena parlare, gli fu detto che non erano credibili le ragioni da me addotte e gli si fece l'alternativa o che fosse entro quattro giorni pagata la somma o che s'intendesse rotta la pace fra il sua Cantone e la Repubblica.

Se vostre Eccellenze hanno finora riconosciuto in me un cittadino attaccatissimo alla sua patria e se mi videro preferir sempre a qualunque particolar riguardo il servigio e gl'interessi dello stato, possono ben credere che strema sia stata la mia angustia alla suddetta ingiustissima proposizione.

Onde cercar pure di avanzar tempo e per apparecchiarlo a ricever col minor irritamento possibile la risposta che mi vedea obbligato di dargli, tentai la strada dei doni e feci a lui e alle principali figure che ha seco distribuir gli effetti che sono nella inserta nota compresi.

Sapendo che parla l'italiano e per fargli vedere che avea per lui una particolar considerazione, glieli feci presentare dal fedelissimo segretario Alberti in compagnia del dragoman Franchini, e poté appena il zelo e la destrezza delli stessi ottener che attendesse l'arrivo del corriere per aver una precisa risposta.

Domani ch'è il giorno alla stessa fissato, tutte usando le avvertenze per non comprometter i pubblici delicati riguardi, mi uniformerò ciecamente alla volontà dell'eccellentissimo Senato, ma temo gli effetti del carattere feroce di costui, delle maligne istigazioni che sarà per fare al suo dey e di qualche molesto risentimento. 
L'Eccellenze vostre, che nella loro sapienza e maturità hanno per certo pesato in tutti i suoi rapporti l'affare, avranno insieme prevedute tutte le possibili sopravvenienze, ma debbo alla mia fede ed al mio onore il non tacer intanto quello che a questa parte mi risulta.

Per ciò non posso neppure tener a mio carico le significazioni replicatamente fatte dal medico del capitan passà al fedelissimo Ralli sopra il desiderio del medesimo di entrar mediatore nell'attuale vertenza con il Cantone di Algeri e benché abbia esso Ralli cercato di destramente sottrarsi da siffatto discorso, nondimeno in una visita recentemente fattagli dal detto medico insistette di nuovo, dicendogli che mi consigliasse a scriver a vostre Eccellenze, mentre il capitan passà erasi più volte chiaramente spiegato che venendo richiesto si farebbe un pregio di conchiudere con prontezza la pace tra la Repubblica e gli algerini.

Qualunque siasi il peso che meritar possono siffatte asserzioni, egli è certo che nella privata visita che ier l'altro ha praticato al detto capitan passà, cercò pur esso di farmi qualche cenno lontano, che lasciai cadere per non entrar in tal dialogo senza pubbliche istruzioni.

Mostrò egli molta compiacenza nel vedermi colmandomi delle maggiori distinzioni e facendomi poscia accompagnar dai suoi uffiziali per veder l'arsenale in cui se all'attività e al numero delle persone corrispondessero l'ordine e la ben intesa direzione, sarebbero considerabili i risultati. Si sta più travagliando a riattar i molti legni vecchi di varia classe e d'imperfetta costruzione di quello che a fabbricarne di nuovi. Rimarcai soltano accantierate una nave a tre ponti, una fregata ed una corvetta. La costruzione di questi legni è modernissima ed è diretta dall'architetto francese Le Brun, ma quantunque siano prossimi ad esser lanciati all'acqua, la nave non potrà mettersi al mare nella prima campagna.

Incomode nell'ultimo ordinario mi giunsero le notizie del console in Smirne. Ei mi avvisa che i mercadanti greci sudditi ottomani hanno ottenuto dalla Porta un firmano per esser esenti dal pagar il dritto del cottimo delle mercanzie che caricano sopra i vascelli mercantili delle franche nazioni. Se non si riesce d'impedirne l'effetto, grave sarebbe il danno che ne risentirebbe la cassa; i consolati sarebbero privi della fonte maggiore dei loro profitti e la pubblica economia potrebbe trovarsi esposta a degli aggravi per sostenerli. Questi ministri forestieri hanno già cominciato a far dei maneggi per cercar che, sulla base delle loro capitolazioni, venga revocato il firmano. Siccome l'articolo delle capitolazioni di pace di vostra Serenità concernente il cottimo è per dir vero alquanto oscuro, così starò osservando l'esito dei suddetti maneggi affinché se riesce favorevole si possa cercar di dare una vantaggiosa interpretazione all'articolo stesso con l'appoggio eziando della consuetudine osservata sino al presente.

Dall'ultime lettere del console dei Dardanelli ho il riscontro che la nave Medea, di là partita li 11 del corrente, dev'esser non molto lontana da Cismè per raccogliere i bastimenti che doveano attenderla al suo passaggio. 
Confidando nell'esperienza e nell'attività del magnifico nobiluomo Bonlini, ho la lusinga che siano stati da lui esattamente adempiti gli ordini che gli si rilasciarono e che sia fra non molto, come desidero, per giunger all'obbedienza del nobiluomo provveditor generale da mar.

Nulla v'ha di nuovo in questa capitale se non l'assassinio succeduto in Cipro del deposto dragoman Moresi da incognite figure eseguito e che si attribuisce a quelli tra i suoi nemici che temevano di vederlo liberato dall'esilio.

Pera di Costantinopoli, li 25 gennaro 1796 more veneto

\section{5}

(Con inserte 2)

$\mathrm{Fu}$, qual me l'era imaginato, grave e molesto il senso prodotto in questo ambasciator tripolino alla risposta ch'io gli fece pervenire per mezzo del fedelissimo dragoman Franchini, incontrando esattamente gli ossequiati comandi di vostra Serenità.

Sentendo che le dimande del suo dey non venivano accolte e che non avrebbe avuti i dinari ch'egli supponeva di ottener senza verun ostacolo, a fronte delle difficoltà da me precedentemente fattegli, proruppe in espressioni di maggior risentimento e dichiarò che se la Repubblica si mancava affatto ad ogni riguardo d'amicizia verso il suo padrone, dovea certamente espettarsi d'esser in simil guisa trattata.

Aggiunse che anco la buona fede veniva tradita, che chiamati dal dey i consoli di tutte le nazioni si erano a gara esibiti di secondar la ricerca, che si volle dal medesimo dare la preferenza a quello di Venezia, il quale replicatamente promise che il denaro sarebbe qui somministrato, che il dey aveva inteso che nell'anticipazione richiesta dovesse comprendersi anche l'annualità già scaduta, la quale non era ancor giunta a Tripoli prima del di lui distacco, che se gli sia stata portata dopo la sua partenza avrà ricusato di riceverla, che se non si era in grado di fornirgli l'intiera somma, almeno si poteva dargliene la metà e che qualora io non credessi di aderire 0 in tutto 0 in parte alla dimanda, volea da me un documento dichiarativo le promesse del console, le ripetute domande, le varie proposizioni fattemi e le ritratte contrarie risposte.

Cercò il Franchini di mitigare con riflessioni il di lui trasporto e singolarmente di esimersi che qualunque somministrazione di soldo non che dalla richiesta carta, ma s'irritò egli vieppiù e scacciandolo da sé con modi aspri gl'ingiunse di significarmi quanto egli desiderava e di riferirgli le mie risposte.

Come però le ducali di vostra Serenità non lasciavano a me facoltà veruna ed era possibile che si volesse maliziosamente carpire l'indi- 
cata carta per abusarne in pubblico danno, così non volendo io entrare in alcun impegno, incaricai questo fedelissimo segretario Alberti di rinovar i tentativi per placarlo, giacché nel primo incontro era felicemente riuscito io a persuaderlo di attendere l'arrivo del corriere. Ebbe però lo stesso la dispiacenza di sentirsi rinovato colla medesima acerbità il primo discorso. Insistette l'ambasciatore sulla promessa avuta e sul dover che vi era di mantenergliela, dicendogli che affidato ad essa era qui venuto senza il danaro che gli occorreva assolutamente atteso l'incarico avuto dal dey di fare a questa parte degli acquisti di valore.

Com'io lo avea istruito espose le ragioni che con dispiacere determinavano l'eccellentissimo Senato a non poter secondare le ricerche del suo padrone, che dovendo io interamente uniformarmi alle pubbliche commissioni, mi si rendeva impossibile qualunque benché minimo esborso, ch'era irregolare ch'io attestassi le promesse del console, che anzi dovevo supporre che non le avesse fatte e che finalmente dovendo a quest'ora essere in ogni cosa istrutto il suo dey per mezzo delle lettere di vostre Eccellenze, l'affare veniva trattato direttamente dai rispettivi governi né i loro ministri potevano più ingerirsene.

Non si mostrò esso in conto alcuno persuaso di tali ragioni e lo incaricò di ripetermi le sue dimande, dicendo che avrebbe inviato il di lui confidente per ricever la risposta. Quando nel giorno dietro comparve il suddetto confidente, tutti posi in opera i mezzi ed i raziocini che il mio desiderio di ben servire ai pubblici oggetti e di allontanare nuove molestie alla veneta navigazione seppe suggerirmi. Facilitarono alle mie parole un ragionavole ascolto 400 piastre e 6 oche di teriaca che gli feci presentare, e parve ch'egli declinasse dalla spiegata pretesa di aver dinaro, ma rinovò la ricerca della più volte accennata carta, paliandone il vero oggetto col pretesto che il suo principale desiderava di aver un fondamento di difesa in faccia al dey, se mai venisse imputato che per sua negligenza e per non aver fatti tutti i passi opportuni non avesse esatti li dinari, mostrando persino di non esser persuaso ch'io avessi ricevute delle istruzioni contrarie alle di lui brame, attesa la mia recredenza nel condiscendere anche ad una semplice dichiarazione in iscritto di quanto era seguito.

Conoscendo però io ad evidenza ch'egli avea dei risoluti ordini dal suo ambasciatore, dai quali non poteva scostarsi, che sembrava non voler ulteriormente insister sopra la somma, e che si ristringeva solo a voler il sopraddetto documento, temetti che una assoluta negativa eziando sopra questo punto non accrescesse l'amarezza del di lui padrone. E poiché sapeva che il dey di Tripoli aveva inviato dei preziosi doni a questo capitan passà, dubitai che non fossero avanzati degli uffizi del medesimo per condurlo a frapporsi nel maneggio e quindi che non m'inviluppasse in un maggior imbarazzo. Pensai pertanto ad uno spediente che in qualche modo potesse soddisfarlo senza 
ch'egli poi ne abusasse dicendogli che gli avrei consegnata una lettera responsiva a quella affidata all'ambasciatore dal vice console.

Dopo molte riflessioni ed insistenze, alfine s'indusse l'accennato confidente a riguardare come non inopportuno il propostogli ripiego, al che contribuì moltissimo la destrità ed insinuazione dell'esperto dragoman Franchini. S'impiegò quindi di farlo gradir anche all'ambasciatore e di venir in seguito a ricever la suddetta lettera, ma sin ora non lo vidi né mi parve conveniente di mostrarmi desideroso di consegnargliela. Se però egli si presenterà gliela rilascerò, avendola estesa con espressioni le più innocue, come vostre Eccellenze rileveranno dalla copia che unisco.

Cercando avendo dal canto mio di uniformarmi alle sovrane istruzioni, desidera che la mia direzione sia stata valevole ad impedire ch'egli faccia al dey di Tripoli del falsi e calunniosi rapporti a pregiudizio dei pubblici interessi.

Ho nei giorni scorsi ottenuta la mia udienza anche da questo sovrano. A tenor del partito preso col rispettabile parere dell'eccellentissimo mio precessore, alla Serenità vostra indicato nell'antecedente numero 13, mi astenni dall'avanzare veruna ricerca sopra i cerimoniali alla Porta, la quale non fece alcuna novità.

Provai la compiacenza che non sia stato considerabile il tempo che attender mi si fece, che i soliti capigì bassì appena si accostarono al mio fianco per introdurmi, che meco entrarono dal sultano altre dieci persone del mio seguito e d'esser stato accolto nella maniera la più graziosa e gentile da questo sovrano, il quale, col mezzo solito del suo visir, rispose con termini d'amicizia ripieni per la Serenissima Repubblica all'offizio che in suo nome gli feci.

La mia condotta in questa occasione fu generalmente approvata da tutti questi stranieri ministri, e cercai anche di farla gradire all'ambasciator di Francia, il quale si mostrò sorpreso ch'io non avessi fatto dei passi analoghi alle di lui insinuazioni per condurre la Porta ad un'alterazione di metodo anche verso il ministro di vostra Serenità, facendone comprendere che senza esserne autorizzato io non poteva promuovere una tal cosa, e attribuendo i modi coi quali venni accolto all'aver egli fatto conoscere al governo l'inconvenienza di alcune antiche usanze.

Con lettera del console di Smirne ebbi con molta compiacenza la soddisfacente notizia che la pubblica nave Medea sia partita dal porto di Cismè nel giorno 23 gennaro prossimo passato, prendendo sotto convoglio ventidue veneti bastimenti mercantili.

Recentemente il suddetto ambasciator di Francia ebbe dal reis effendi una particolar udienza, che durò lungamente. Varie congetture sopra ciò furon fatte, ma però comunemente si crede aver egli avuto per oggetto principale il distoglier la Porta dalla credenza in cui la supponeva, che stretti fossero i legami tra la Svezia e la Russia, e che si fosse la prima dalla Francia alienata, su di che vostra Sere- 
nità avrà dai ministri rispettivi lumi più precisi per conoscere i veri attuali rapporti delle dette potenze.

Sono di qua partite le due fregate francesi, ma ci rimase la truppa di artiglieria leggera entrata al soldo di questo sovrano.

Tutti gli emigrati di quella nazione qui esistenti, che portavano la croce di san Luigi, di cui erano decorati, furono per ordine espresso della Porta obbligati a deporla, giacché con promemoria ne fece appositamente la dimanda questo ministro repubblicano, irritato tra gli altri contro certo Monsieur Ghismond, protetto dall'inviato di Napoli, a cui egli stesso rimarcò siffatto distintivo dipendente dall'abolita monarchia.

Non sono più soli i bastimenti russi ed imperiali che godono del privilegio di caricarsi di grani in questo porto; lo ricercò pure il console di Ragusi e, dopo vari maneggi e molto dinaro impiegato, ottenne alla sua nazione questo considerabile vantaggio.

Pera di Costantinopoli, li 10 febbraro 1796 more veneto

P.S. Giunto il corriere dopo esteso il presente, ricevo le venerate ducali 31 dicembre e 7 gennaro decorsi. I sensi di quest'ultima furono di sommo conforto all'animo mio nel rilevare con quanta clemenza siasi degnata la pubblica autorità di accogliere i primi riscontri dell'assunto mio incarico, e saranno alla mia obbedienza presenti le prescrizioni di cui vengo dalla Serenità vostra onorato nelle ducali medesime.

\section{6}

(25 febbraro 1796 more veneto)

Dall'ossequiata ducale 21 gennaro passato rilevo pervenute alla Signoria vostra le carte che riuscì alla diligenza del console di Smirne di ritrarne relazione alla molestia con gli algerini. Mi astenni dal procurare che il capitan pascià autenticasse i suddetti documenti, come presagiva il console in Algeri, perché in vece di limitarsi ad una semplice assegnazione, che difficilmente avrebbe accordata, si sarebbe forse indotto a credere che sulle insinuazioni fatte dal di lui medico al Ralli, e indicate nel mio riverente numero 14, potesse esser ricercata la sua mediazione, al che non mi sarei giammai esposto senza positive prescrizioni di vostra Serenità. Questa necessaria circospezione ho il conforto di riconoscerla conforme alla ducali 7 gennaro, responsive al cenno fatto sull'influenza del predetto ottomano ministro, colle quali in questo argomento vengono riconfermate le generali pubbliche massime. Scrupoloso io di non istaccarmene dalle medesime senza essene autorizzato, l'Eccellenze vostre avranno riconosciuto eziando dal mio numero 15 come cercai di al- 
lontanare i motivi che l'ambasciatore di Tripoli si rivolgesse al detto capitan pascià per conseguire le ricercate dichiarazioni in iscritto, e gli abbia perciò promessa la lettera, onde sottrarmi da ogni imbarazzo. Questa fu anche consegnata sulle nuove domande ch'egli mi fece giungere, non senza incontrar però delle resistenze per la semplicità con la quale l'avevo espressa, mentre gli parea strano che ricusata da me qualunque somministrazione di danaro volessi usar senza riserva nel corrisponderlo di poche parole.

Concepita appena la lusinga di aver dato termine ad ogni affare con questo affricano, fu dallo stesso posta a campo una nuova molestia. Pervenne a mia notizia che cercava di noleggiare due bastimenti veneti, non solo per tradurlo a Tripoli con il suo seguito ma ancora per trasportare i regali ricevuti dalla Porta per il dey, consistenti in munizioni da guerra e legnami da costruzione. Volendo sopra questo interessante punto appoggiare la mia condotta sopra sicure norme, presi in esame le sovrane deliberazioni de' scorsi tempi in consimili circostanze. Mi risultò dunque che col decreto 15 maggio 1783 fu prescritto al bailo e ai consoli de' porti dove facili e frequenti esser possono le ricerche di noleggi per parte delle Regenze affricane, che senza far apparire veruna pubblica ingerenza, o rappresentanza della loro figura, venisse prudentemente insinuato ai capitani di prestarsi, salvo il loro interesse, a tan natura di contratti, per evitar così i disgusti e il risentimento de' Cantoni barbareschi che si dolevano della loro resistenza. La ducale 21 aprile 1784 approvò in appresso la condotta del bailo d'allora, il quale per non indisporre il capitan pascià ed il dey d'Algeri, non si oppose alla partenza da qui di un bastimento carico di munizioni per quella reggenza, ma gl'ingiunse insieme che continuando l'ostilità della Spagna contro gli algerini dovesse destramente esimersi da siffatte domande per non offendere l'amicizia sussistente con detta corte e mantenere la neutralità che conveniva al sistema della Repubblica.

Da queste e da molte altre successive ducali, potei quindi dedurre che indiferente l'eccellentissimo Senato, ed anzi tramando che i legni sudditi siano con qual si volgia carico noleggiati per le Reggenze affricane, quando queste sono in pace con le potenze amiche, non vuole che ciò segua allorché sono in guerra.

Sussistendo però attualmente le ostilità della Danimarca contro il Cantone di Tripoli, le pubbliche sapientissime deliberazioni si oppongono alle ricerche del predetto ambasciatore, e quindi cercai con ogni più destro modo d'impedire la verificazione di quanto meditava ingiungendo ai capitani che con pretesti unicamente privati di antecedenti noleggi, di commissioni di parcenevoli e simili si sottraessero da ogni impegno. Feci loro eziando delle serie minaccie affinché usassero su di ciò la maggior segretezza, ma con mio sconforto debbo rassegnare l'inutilità di tali precauzioni, per effetto della loro insubordinazione e della scaltrezza dell'ambasciatore, imperoc- 
ché chiamato da questi il dragoman Franchini, con sensi del maggior irritamento gli disse ch'erasi accorto della misteriosa ed artifiziosa condotta del bailo di Venezia, il quale tendeva di occultamente opporsi alle sue brame, che dalla serie delle cose corse e dal mio procedere scorgeva chiaro che si voleva romperla col bey, che gli abbisognavano due bastimenti per trasferirsi a Tripoli, che prima di qui giungere, trovandosi a Smirne, avea commesso al suo agente di noleggiarne uno, come gli riuscì con certo capitan Bronza, che in breve arriverebbe in questo porto, e che se non volessi accordargli un secondo legno non avrei potuto impedire che si valesse di quello del Bronza senza venire ad un'aperta nemica spiegazione.

Sorpreso da tal discorso, ricercai l'esperto dragomano di procurarsi un nuovo colloquio con il medesimo, di cercar di allontanare ogni suspizione della mia condotta, di fargli conoscere che si troverebbe poco sicuro sopra un legno di veneta bandiera, massime se carico di munizioni da guerra incontrasse la squadra danese, ch'era voce comune si dirigesse a bloccare il porto di Tripoli, e di lasciargli travedere in fine che i veneti capitani non dovevano essere senza timore, tanto nell'andata che nel ritorno, attese le differenze insorte tra la Repubblica ed il Cantone di Algeri.

Vedendo quindi l'ambasciatore da tanto ciò che io mi interessavo anche per la di lui sicurezza, parve restar persuaso delle mie riflessioni e men fiero di prima assicurò il Franchini che mediante un robusto firmano procuratogli niuna molestia sarebbe inferita dagli algerini, soggiungendo di più che siccome questo incaricato d'affari di Danimarca avea interposto il capitan pascià per ristabilire la pace tra il dey e il suo re (cosa di cui me ne sono assicurato per altra parte), così era certo che per obbligarlo a cooperare egli pure a questo fine, non gli sarebbe ricusato un passaporto per essa e per i legni che lo seguissero.

Promise il Franchini di riferirmi ogni cosa, ma che intanto credeva di doverlo prevenire che quand'anche il ministro di Danimarca gli accordasse un passaporto, era necessario che fossero espressamente dichiarati in esso gli effetti che seco voleva condurre, altrimenti né egli poteva ritrovarsi sicuro né il bailo assentirebbe che niun capitano li caricasse, perché essendo la Repubblica amica del dey e di sua Maestà danese, il permettere un carico di munizioni da guerra sopra bastimenti veneti era contrario ai principi di perfetta imparzialità che professava sulle vertenze tra loro sussistenti, locché trovato ragionevole dall'ambasciatore soggiunse che conseguito il passaporto me lo farebbe tenere acciò lo esaminassi per mia tranquillità.

Io credo assai difficile che il ministro danese sia per farsi responsabile in faccia alla sua corte quando non avesse delle speciali facoltative, ed infatti quantunque trascorsi vari giorni non ancora comparve la persona col promesso passaporto. Ma se mai riuscisse all'ambasciatore tripolino di ottenerlo, quale dalla destrità del sud- 
detto dragomano Franchini gli fu suggerito, cessando in tal caso ogni obbietto, crederei di aver ben servito alle pubbliche mire in ogni rapporto, lasciando procedere il contratto senza però prendere alcuna diretta ingerenza né nelle condizioni del medesimo né nell'obbligare i capitani a verificarlo.

Ricercata dall'eccellentissimo mio precessore un'apposita spedizione a Corfù, com'egli ha già rassegnato a vostra Serenità, mi parve di dover aderirvi in grazia dell'oggetto propostosi, ed avendo periò incontarta la spesa di piastre centonovantacinque, supplico per la relativa sovrana approvazione.

Di gradimento riuscirono i metodici regali fatti al sultano, alle favorite all'altre figure secondo l'usato. Nelle dispense tanto ordinarie che straordinarie degli stessi, ed anche nelle mancie sborsate essendomi come dall'inserta nota apparisce scrupolosamente attenuto alla norma dei precessori, io confido che dalla pubblica autorità saranno benignamente assentite.

Nella scorsa settimana giunse da Petersburgo uno straordinario corriere a questo inviato di Russia che gli recò la gran croce dell'ordine di Sant'Alessandro e nell'istesso tempo la di lui elezione a consigliere intimo della corte ed il titolo di tenente generale.

L'arrivo a questa parte dell'ambasciator persiano, che accennai nel precedente numero 12 , dicesi che sia rincresciuto al suddetto ministro e che abbia egli chiesto formalmente alla Porta di congedarlo senza dargli ascolto. Pretendesi quindi che soddisfatta una tale ricerca sia l'ambasciatore prossimo a partire, ma che per altro occultamente il governo sostenga gl'interessi del vecchio Mahamud Kan.

Appiccatosi il fuoco nel borgo di Galata poco distante dalla scala franca nella notte dei 18 corrente, furono sì rapidi e violenti i progressi delle fiamme che in meno di cinque ore rimasero incenerite oltre cinquecento case, nella qual occasione fu incredibile l'attività del capitan pascià.

Martedì passato furono lanciate nell'acque i tre legni da guerra, che come indicai in altro numero si trovavano accantierati in questo arsenale, e tutto riuscì a seconda delle misure prese dall'abile costruttor francese Le Brun. Vuolsi che il capitan pascià gli abbia regalato 4 mila piastre di denaro, ed una pelliccia del valore di mille, ma che sia rimasto poco soddisfatto perché né il sultano né gli altri suoi principali ministri gli manifestarono sin ora il loro gradimento con visibili contrassegni.

Compitosi sino dagli undici corrente il primo trimestre del mio arrivo a questa parte, ho rilasciato all'ossequioso nome di vostra Serenità la solita cambiale di reali 7500 , rimessa al mio procuratore conte Giuseppe Mangili, della quale ne imploro l'accettazione e pagamento alla sua scadenza. 
Più volte ne' scorsi tempi fu alla Serenità vostra rappresentato dai miei precessori quanto i schiavoni qui dimoranti riescano inquieti perturbatori del pubblico riposo, e indocili agli ordini della carica. Con siffatto molesto argomento debbo io pure, mio malgrado, trattenere l'eccellentissimo Senato, avendo avuto motivo ne' passati giorni di esercitare sopra di ciò la mia più attenta vigilanza.

Sussistendo delle differenze tra un bostangì ed un suddito dalmatino, condotto questo dal suo violento carattere si portò a deciderle da se stesso con l'uccisione del bostangì suddetto. Eccitato il governo, oltreché dall'azione malvagia anche dalle querule e dalle lagrime de' parenti del defunto, mi fece avanzare le più risolute lagnanze chiedendo un pronto e severo castigo al reo, con minaccia persino di far imprigionare tutti i sudditi schiavoni.

Per dare una prova non equivoca della giustizia che la Serenità vostra vuole rigorosamente esercitata da chi ha l'onor di rappresentarla in questa corte, per offrir nello stesso tempo una soddisfazione dovuta al fondato reclamo e per impedire un passo che nelle determinazioni precipitate di questo ministero comprometter poteva la pubblica dignità, promisi con ogni prontezza di procurar il rinvenimento, l'arresto e l'esemplare castigo del colpevole, rilasciando a tal caso gli ordini più pressanti.

Partecipate alla Porta le prese misure dal fedelissimo dragomano Ralli, le fece gradire alla medesima colla sua destrità, per cui fu intanto sospeso l'imperial comando di arrestar generalmente i schiavoni, avendo nel tempo stesso maneggiato il bostangi bassì, promotore principale delle violenti risoluzioni che abbracciar si volevano. Sino ad ora però vana si fu ogni indagine per iscoprire il reo, il quale se continui a rimaner celato ed a sottrarsi alla vigilanza della carica, non so come si potrà acquietare la Porta, che sta in attenzione per veder l'effetto delle mie promesse.

Una prova di ciò sono le ricerche quasi giornaliere del reis effendi al Ralli e la spiegata sua domanda d'aver la nota degli schiavoni patentati, indicando che l'eccellentissimo precessor cavalier Foscarini convenne precisamente col governo di non rilasciare la patente che a soli trenta individui.

Cercai quindi che il suddetto dragomano Ralli si sottraesse destramente dal produrre essa nota, e gl'ingiunsi di far riflettere al reis effendi che per i trenta schiavoni patentati secondo l'anzidetta convenzione, non si poteva intendere se non se quegl'individui della stessa nazione che occorrono alla carica; ch'era impossibile che l'eccellentissimo bailo d'allora avesse preteso di rinunziare assolutamente alla facoltà di far riconoscere e proteggere, qualor se ne rendessero degni, un numero maggiore di tai sudditi veneti, i quali per i loro particolar interessi, o per altri giusti motivi, si portano negli 
stati ottomani; che in oggi la quantità n'è riflessibile, perché molti sono addetti al servigio di case private ed anco di stranieri ministri, locché prova la loro moderazione e disciplina; che non era possibile de qui di trarne il numero variabile tutto giorno secondo le cause che li determinano ad occorrere o ad allontanarsi di qui; che dovendosi rinovare tra quattro mesi le patenti impiegherei ogni cura per diminuirle possibilmente, premettendo i più scrupolosi esami avanti di rilasciarle e che infine le ricuserei a tutti quelli che non comproveranno senza equivoco la loro buona condotta e sudditanza, facendoli partire o abbandonandoli alla giustizia del governo.

E poiché questo è il solo spediente, uniforme eziando alla volontà di vostre Eccellenze, per minorare almeno in qualche parte alla carica i fastidiosi imbarazzi ne' quali trovasi di frequente avvolta per opera di torbidi individui, mi adopererò con tutto l'impegno per soddisfare a quanto fece promettere al reis effendi, limitando nei modi possibili il numero dei patentati schiavoni, non mai circoscrivibile per altro a soli trenta, giacché tra quelli che sono indispensabili a questa legazione e gli altri che da continui uffizi si trova costretta di permetter che passino al servigio di stranieri ministri e de' loro dipendenti, la quantità n'è molto maggiore.

Sebbene, come indicai in altro riverente numero, ne abbia allontanati diversi in occasione della partenza per Corfù della nave Medea, dovendo sovente sì di questi che di altri sudditi trattenerne qualcuno in queste pubbliche carceri, ed essendo le medesime ridotte mal sicure per la debolezza della fabbrica, e perniciose alla salute dei detenuti a grado che non era esente dalle pessime esalazioni questa casa di vostra Serenità, a cui sono prossime, mi fu forza accorrere alla loro istantanea riparazione, che verificata con ogni possibil risparmio importò reali 547, di cui ne imploro la sovrana approvazione.

Seguita l'anno scorso l'elezione di Hachì passà in beylerbey di Romelia con ampla autorità ad oggetto di estirpare i ladri che in gran numero infestano quella provincia, seguendo esso gl'impulsi del suo carattere sanguinario e feroce, e confondendo i rei cogli innocenti, scagliò il suo furore sopra di tutti indistintamente, e si rese insoffribile per modo che avanzati al governo i più forti reclami contro di lui, s'indusse a sospendere la commissione ed a richiamarlo in Adrianopoli. Si crede che verrà deposto, benché siavi chi assicura ch'ebbe la sorte di discolparsi e che gli sarà conferito il comando di un grosso corpo di truppe che si va in quelle parti raccogliendo.

Pretendono poi alcuni che un tal armamento abbia per iscopo la presa risoluzione della Porta di distruggere affatto i suddetti malviventi e di ridurre all'obbedienza certo Pasvandohuglù, che n'è il capo; ma altri giungono a supporlo un pretesto e che il divano si sia proposto di tener in prossimità ai confini della Servia in corpo di armata per imporre alla Casa d'Austria facendogli sospettare qualche intrapresa contro i suoi stati per favorire le viste della Repubblica Fran- 
cese e condurla così più facilmente a pensieri pacifici. Se questa ultima congettura sia la più probabile, il fatto non dovrebbe tardare a dimostrarlo, essendo nondimeno per ora assai incerta, perché si sa che gli avveduti e pressanti maneggi di questo ambasciator francese per indurre la Porta a delle risoluzioni corrispondenti agl'interessi della sua repubblica, locché sembrava l'oggetto principale della sua missione, non ebbero quell'esito ch'egli forse si era proposto.

Il passallaggio di Scutari non è stato più diviso in tre distretti, com'era prima intenzione della Porta, e come mi onorai d'indicare nel precedente numero 7, ma venne concesso interamente ad Ibrahim passà, fratello dell'estinto Muhamud, a ciò indotta dal riflesso di allontanare le male intelligenze che destar poteva la divisione del comando, dai saggi di assoluta dipendenza e di fedele attaccamento manifestati in ogni tempo dal suddetto Ibrahim e dall'offerta, forse la più persuadente di ogni altro motivo, di due mila borse, avendone contato frattanto 650 Saffian agà, suo capì kiaya, che in di lui nome ricevette l'investitura del passallaggio nel giorno 4 corrente.

Pera di Costantinopoli, li 10 marzo 1797

P.S. Pervenutami prima di dar spedizione al presente l'ossequiata ducale 4 febbraio decorso in riscontro delle mie riverenti numeri 9 e 10, rileva l'animo mio i clementissimi sensi dell'eccellentissimo Senato, il di cui sovrano generoso compatimento servirà sempre a confortare l'instancabile mio zelo nel servire ai pubblici venerati cenni in questa difficile legazione.

\section{8}

(Con una inserta)

Com'ebbi l'onor di assoggettare nel riverente numero 16, questo incaricato di Danimarca ricusò di rilasciare all'ambasciator tripolino il chiesto passaporto dichiarativo la qualità dei generi che caricar volea sopra i veneti bastimenti. Sospesa quindi per alcuni giorni la trattazione dell'affare, giunse nel frattempo la notizia per la via di mare della conclusion della pace tra sua Maestà danese e la Reggenza di Tripoli. Dal modo con cui meco si espresse il suddetto incaricato, sembra c'egli vi presti fede, ma non avendone ricevuto avviso ministeriale non volle prudentemente impegnarsi a segnare l'anzidetto passaporto.

Questa riserva indusse il tripolino a noleggiare un bastimento francese, onde caricarlo per quanto si assicura con bandiera turca di tutti quegli attrezzi e generi da guerra e farlo partire quando sarà sicura la pace con la Danimarca, ed a sceglierne uno veneto per 
esso e per il suo seguito e scioglier da questo porto sollecitamente. Comunicatami siffatta sua determinazione, siccome non trovai più obbietto nel secondarla, così mostrai desiderio che tra i legni di veneta bandiera ne rinvenisse uno corrispondente alle sue viste. Gli feci però rimarcare che la riserva spiegata dal ministro danese, sinché non gli perveniva ufficialmente la notizia della pace, dovea averla io pure, e che perciò il capitano esprimerebbe il contratto di non caricare effetti di guerra. Convenuti anche sopra questo punto non poteva giammai imaginare che quegli che rappresentava vostra Serenità dovesse pensare a trovar il bastimento e a stabilire il relativo noleggio. Ma contro ogni mia aspettazione tale appunto si fa la domanda spiegata in appresso dall'ambasciatore, col pretesto che più agevole a me sarebbe di convenire col capitano e che più eziando discreta riuscirebbe la ricerca del medesimo qualora il contratto fosse proposto dal bailo.

Vedendo che cercava d'impegnarmi a far condiscendere il capitano a delle misure forse non eque ch'ei meditava, gli feci riflettere che trattandosi d'interesse particolare né per giustizia, né per le leggi del mio governo poteva ingerirmene, né usare alcuna violenza. Soggiunse allora che scorgeva evidentemente non voler io aderire in nessun modo alle sue brame e manifestatosi al sommo irritato mi convenne, per non indispettirlo di più, offrirgli di far chiamare quello fra i capitani ch'egli desiderasse, d'insinuar al medesimo di cogliere l'occasione di servirlo e di essere discreto nelle sue domande. Quanto poi alle condizioni gli aggiunsi che spettava al di lui confidente lo stabilire nell'offizio della segreteria, dove si stipulano i contratti alla presenza del segretario della carica. Persuaso di ciò, insorsero nuove difficoltà per parte dei capitani. Quegli stessi che dapprima o indocili alle fattegli insinuazioni, o poco avveduti per conoscer gli artifizi del tripolino, aveano dato ascolto alle di lui proposizioni, compromettendo i pubblici riguardi, ricusarono di noleggiare i loro legni, adducendo che non volevano esporsi al pericolo, arrivati a Tripoli, di perdere oltre la somma che convenissero, forse anche la libertà e le sostanze. Ciò ebbe origine dall'essersi l'ambasciatore espresso ch'era poco contento della direzione de' veneti verso il suo dey, non che dal ritratto fattone come di uomo violento, feroce e rapace dal capitano svedese che qui lo condusse, e che ricusò poi di dargli di nuovo imbarco per aver dovuto soggiacere ad una sensibile minorazione del pattuito noleggio.

Era tale il panico timore generato da siffatte disseminazioni che per più giorni riuscì vana ogni mia cura per persuadere i capitani ad abbracciare il partito. Ricusavano questi dall'una parte di assumerne l'impegno senza un espresso comando della carica e senza la pubblica garanzia, e dall'altra l'ambasciatore giudicando artifiziosa e derivante dalle segrete mie commissioni la loro ripudia, minacciava di vendicarsene in modo il più risoluto. 
Combatteva il mio spirito tra il dubbio che l'affare terminar potesse con un'aperta rottura anche con il Cantone di Tripoli e tra il pericolo d'impegnar il pubblico in una risponsabilità senza limiti, quando alfine il capitan Lambrinò Panà, comandante la nave Penelope, dando una prova di vero suddito zelo e disinteresse, s'indusse volontariamente a stipular il contratto col confidente dell'ambasciatore per una summa discreta e con tali condizioni da non compromettere i riguardi di vostra Serenità, avendo ricercato in oltre che dallo stesso gli fosse procurato un buiurdì del capitan passà per garantirlo dagli algerini, contratto per altro non ancora ratificato dall'africano ministro.

Merita in vero un elogio la condotta di questa capitano, giacché i timori dagli altri e da lui stesso spiegati pur troppo non sono senza fondamento, in grazia della mala fede de' barbari, del risentimento che mostra l'ambasciatore per non aver ottenuta la chiesta anticipazione, degli ostacoli frapposti quando caricar voleva sui veneti bastimenti munizioni da guerra, delle difficoltà che incontrò per isfortuna l'ultimo contratto e dal manifesto suo torbido e fiero carattere. Un altro motivo evvi ancora per cui si può dubitare che giunto in Tripoli sieno esagerate ed infedeli le sue relazioni al dey, nell'animo del quale è comune voce che sia influentissimo e che perciò il capitan sia esposto a danni e vessazioni, o l'eccellentissimo Senato a qualche inattesa molestia.

Avea egli fatto noleggiare alla Smirne, come indicai a vostre Eccellenze, il capitan Bronza, il quale, per quanto asserisce l'affricano, dovea partire da quel porto dieci giorni dopo. Non essendo ancora arrivato, l'ambasciatore, che unicamente per i suoi affari si è qui trattenuto, sostiene ora che si fermò quaranta giorni di più di quello gli abbisognava per aspettarlo e che perciò deve indennizzarlo. Supponeva egli che il ritardo procedesse dal mio ordine, ma avendolo fatto assicurare ch'io ne ignorava il motivo, soggiunse che sapeva il modo di risarcirsi. Cercai d'indagare quali fossero i suoi disegni, ma si occultò sempre dicendo che non dovea il bailo occuparsi, sicché nell'incertezza delle sue mire sembra ragionevole il dubitare ch'esso mediti qualche molesto tentativo.

Ho scritto subito al console in Smirne per aver l'istruzioni che mi mancavano e quando sarà vicina la partenza dell'ambasciatore procurerò di blandirlo col farlo complimentare dal fedelissimo segretario Alberti, come praticai al di lui arrivo, presentandogli qualche nuovo dono.

Siccome era mio dovere di rassegnare ogni più minuta circostanza di questo ingrato argomento a lume della pubblica sapienza, così mi perdonerà l'eccellentissimo Senato se osai distrarlo da più gravi cure col troppo diffondermi a questa riverente esposizione.

Venera l'ossequio mio la sovrana ducale 18 febbraro decorso, dai sensi della quale desume nuovo conforto il mio zelo, scorgendo la continuazione del pubblico generoso compatimento e attenderò in con- 
formità della medesima lo species facti sopra gl'avvenimenti dell'estere armate in Italia per farne un cauto ed avvertente uso nelle circostanze già alla Serenità vostra rassegnate.

Entrato il possesso della sua carica il gran doganiere nel giorno 12 marzo corrente, mi sono uniformato al contegno de' miei precessori in simili occasioni ed all'esempio degl'altri stranieri ministri, facendogli pervenire i consueti doni di metodi espressi nella inserta nota, che abbisogna della pubblica approvazione.

Trovasi pronto a far vela il bastimento che trasportar deve il turco ambasciatore a Parigi, affrettato dal governo a sollecitare la partenza. Sarà egli accompagnato dal francese Vantour, ch'esercitava qui l'offizio di primo dragomano della sua legazione e ch'è dal Direttorio destinato maestro di lingue orientali all'Istituto nazionale, non che dal giovane offiziale Colincourt aiutante di campo del general Du Bayet.

Ieri diede fondo in questo porto l'ottomana squadriglia che nello scorso inverno si tenne a scorrer le acque dell'Arcipelago, avendo seco una preda maltese.

Pera di Costantinopoli, li 24 marzo 1797

\section{9}

(Con inserte 12)

Erettosi provvisionalmente un teatro nella città di Smirne, rimase nel giorno 12 del decorso mese ucciso alla porta del medesimo un giannizzero per opera di un ceffaloniotto, ch'era protetto dalla Russia, per quanto accenna il veneto console, e da ciò ebbe origine la funesta catastrofe che forma dolentissimo argomento nel presente dispaccio.

Riuscite inutili le perquisizioni dei giannizzeri e dei governatori ottomani per rinvenire l'omicida, e rimaste essendo senza effetto le ripetute domande fatte dal medesimo ai consoli di tutte le nazioni, i giannizzeri furibondi appiccarono il fuoco in vari angoli della franca contrada, assalirono le case consolari, saccheggiarono indistintamente i magazzini e le abitazioni dei franchi ivi residenti e rapirono le sostanze, spargendo con inaudita barbarie in ogni parte la desolazione ed il terrore.

Mancato sarebbe ai franchi ogni asilo se avventurosamente i navigli mercantili ch'erano in porto non li avessero accolti, somministrando così un rifugio a quei che poterono salvarsi dalla sanguinaria ferocia de' sollevati.

Nella notte de 25 marzo giunsero alla carica ed agli altri esteri ministri le relazioni sul tragico fatto, spedite con espressi corrieri dai rispettivi consoli i quali, in mezzo alla costernazione ed all'orrore, si erano essi pure salvati sopra i navigli. 
Dalle inserte lettere numeri 1 e 2 del console veneto rileverà la Signoria vostra ch'egli asserisce che era coperto dalla protezione imperiale il teatro il di cui ingresso contrastato all'omicida fu l'origine dell'orribile fatto accaduto; che l'uccisore stesso era protetto russo; che ciò sapendo il governo ottomano lo richiese al suo console; che quantunque cercasse questi che le domande fossero rivolte al veneto, nessuna gliene fu avanzata; che nella sessione tenutasi alla presenza dei comandanti turchi da tutti i dragomani consolari, nella quale si rinovò la ricerca prima del console russo, e indi tutti indistintamente, propose il veneto che si facesse dalle guardie turche la visita ai bastimenti di veneto paviglione per iscoprire se vi fosse nascosto il reo, e che infine i giannizzeri, dopo tre giorni di sofferenza, incendiarono il borgo franco, investirono in particolare la casa del console di Moscovia e fecero fuoco sopra coloro che colla fuga si ritiravano alla marina.

Non è esprimibile la commozione cagionata dall'annunzio di tante stragi e rovine, la quale nondimeno veniva in qualche modo temperata dalla lusinga che essendo l'omicida, benché di origine veneta, protetto dalla Russia, riconosciuto eziando tale dagli ottomani con la ricerca fattane al console di quella nazione e per le violenze praticate in preferenza alla di lui casa, non sarebbe il governo per dessumere dall'accaduto un motivo di turbare i pubblici riguardi, di quantunque per altro risultasse censurabile la direzione di tutti i consoli per non aver impiegato ogni mezzo possibile ad oggetto di calmare l'irritamento degli ammutinati, giacché ebbero tre giorni di tempo per tentarli.

Di breve durata si fu però la concepita speranza, mentre nel giorno 27 decorso questo inviato di Russia con l'annessa nota numero 3, estendendo a due individui l'origine del misfatto, indicò ch'essendosi sparsa voce che appartenessero alla Russia i rei dell'ucciso giannizzero, dichiarava formalmente ch'era un'aperta calunnia del veneto console l'averli per tali indicati, forse come un mezzo di discolpa nazionale o personale, e chiedeva perciò soddisfazione e castigo del medesimo.

Per questa rimostranza pertanto se rendasi contingente la verità delle relazioni del console veneto, dubitar potevasi nel medesimo tempo che il suddetto inviato cercasse di sollevare il proprio console da ogni responsabilità

In argomento cotanto grave, dopo le più mature considerazioni fatte unitamente all'eccellentissimo mio precessore, che col suo riputato consiglio fece scorta ad ogni passo, ed avvalora la presente esposizione colla rispettabile sua firma, fu trovato opportuno di estendere le tre carte che si assoggettano alla sovrana sapienza dell'eccellentissimo Senato ai numeri 4, 5 e 6. Consiste la prima nella memoria prodotta dalla Porta perché fossero impediti gli ulteriori disordini e ristabilita la pubblica tranquillità in quella scala, uniformandosi in 
tal guisa all'esempio degli altri ministri perché un assoluto silenzio per parte della sola Repubblica avrebbe fatto supporre timore nella carica ch'esser potesse l'origine del male ai veneti attribuita. In mancanza di precisi lumi, per non azzardare dei fatti senza fondamento, si conformò nella maniera più semplice ond'essere in stato di aggiungere in appresso tutto quel più che dalle successive notizie fosse per risultare. La seconda si è una lettera spedita per espresso al console colla quale gli vengono significate le doglianze del ministro di Russia e il dubbio nato sulla sincerità delle sue relazioni; gli si ricercano perciò circostanziati dettagli dell'accaduto sulla base di veridici documenti e gli si rilasciano istruzioni per contenere i sudditi nella dovuta disciplina. Nella terza infine viene formata risposta all'inviato di Russia, assicurandolo che sarà preso in esame l'affare e che a norma delle risultanze si procederà rigorosamente verso il console, la qual risposta colla relativa nota sarà comunicata per lume all'eccellentissimo nobile in Pietroburgo.

Premesse queste disposizioni, le sole che le circostanze del momento potevano permettere, nuovo soggetto d'imbarazzo derivò alla carica nel giorno 30. Si produsse con insolita formalità una persona espressamente spedita dal dragomano della Porta dicendo che al solo bailo ed in proprie mani doveva consegnare la carta, che si rassegna al numero 7. Questa, come lo rileveranno l'Eccellenze vostre, â due parti: l'una riguarda il tragico successo di Smirne e l'altra versa sopra un fatto che per la sua intelligenza merita qualche storico dettaglio. Un capitano di origine veneta, ma da molti anni addetto al servigio della Russia, essendo stato citato da un kagià ai tribunali ottomani e vedendosi privo della russa protezione si dichiarò suddito veneto. Chiamato alla Porta il dragoman Franchini rappresentò che il detto capitano non era dal bailo considerato come suddito perché avea sempre servito la Russia, e che perciò lo abbandonava al governo. Ordinatone da questo l'arresto, fu spedito un ichokadar dal reis effendi per eseguirlo. Riuscì anche allo stesso di prenderlo, ma sopragiunti alcuni marinai, i quali sdegnando di veder fatta quasi sotto i loro occhi simile esecuzione, lo tolsero a forza dalle mani dell'uffiziale turco, che fu anche battuto e spogliato di vari effetti. Irritati i ministri della Porta per siffatta violenda dimandarono con modi vigorosi e risoluti la consegna del capitano e dei marinai, qualificandoli per veneziani senza però sicuri fondamenti. Si rinnovarono allora le proteste, che il capitano non apparteneva al ministro di vostra Serenità, che sarebbero rigorosamente puniti i marinai qualora venissero indicati e fossero veramente veneti, e che di più sarebbe impiegata ogni cura per iscoprirli ed arrestarli.

Ad indisporre però maggiormente la Porta giunse nel frattempo l'infausta notizia di Smirne. Era riuscito ai ministri della cancelleria russa di aver in loro potere l'anzidetto capitano e invece di rimetterlo alla Porta che lo avea chiesto, lo fecero passare, senza partecipar- 
lo alla carica, al dragomanno Franchini il quale, sapendo le dichiarazioni avvanzate al ministero ottomano, credette di non esser tenuto a riceverlo. L'inviato di Russia nel tempo stesso significò alla Porta che avea fatto consegnare il capitano ai veneti e che da questi si dovea ripeterlo, e ciò appunto â dato motivo al secondo articolo della memoria sopraindicata.

Grave l'affare per se stesso trattandosi di violenze praticate ad un turco ufficiale da marinai asserti veneti e di un uomo che la Porta ricercava e che offerto non era stato ricevuto da un veneto dragomano, divenne, come sopra si è detto, di più riflessibile conseguenza dopo l'accaduto in Smirne. S'imputavano i veneti in tutto: si accusavano di voler sottrarre i colpevoli dai meritati castighi, venivano di mala fede sospettati e nel mentre i rei di Smirne si esigevano risolutamente dal console, dal ministro di vostra Serenità e dalla Repubblica, si voleva poi dal bailo in particolar l'indicato capitano.

Questo complesso di circostanze facea temere a ragione le più decisive risoluzioni da parte della Porta se non si cercava di calmare almeno in parte l'ottomano ministro consegnando il capitano replicatamente chiesto. Diretta quindi ogni cura a tal fine, che in quel momento parve la più essenziale, e tutt'impegnando i mezzi voluti dalla difficoltà della casa, sortì d'averlo nelle mani, non senza sacrificio e fatica, poscia che le mire della legazione russa sembravano tendenti a sottrarlo alle perquisizioni della carica.

Verificata la consegna ed estinto alcun poco quel primo impetuoso risentimento, si è formata la risposta alla memoria del dragomano della Porta, sulla quale eransi di già prese delle misure preventive inviando con espresso corriere al console di Smirne la lettera annessa al numero 8, colla quale si rilasciarono al console ordini precisi per procurare se sia possibile l'arresto dei rei fuggitivi e per trasmettere alla carica i necessari documenti. Frequenti e risolute furono le prescrizioni fatte in più tempi al console stesso affinché impegnasse ogni sua cura per contenere nella dovuta moderazione que' sudditi.

Nello scorso ottobre poi emanò un imperiale firmano col quale venne ordinato ai comandanti di Smirne di fissare uno stabile regolamento per assicurare il buon ordine e il pubblico riposo in quella città. Ignora però la carica se questo abbia avuto luogo, ovvero se siano rimaste senza effetto le prescrizioni della Porta per indolenza dei comandanti ottomani, come altresì non è abbastanza documentato dalle relazioni precedenti del console se per prestarsi a cooperare per parte sua all'esecuzione del firmano abbia, come disse in sua lettera 21 gennaro decorso, privato della pubblica protezione que' malviventi abbandonandoli al governo, unico mezzo per tenerli in freno. Ma quando anche ciò fosse stato verificato non constava poi se il ceffalonioto omicida fosse o no compreso tra i sudditi abbandonati.

Circondato però da tanti dubbi l'affare, si formò l'unita nota numero 9 concepita in modo da non compromettersi con positive asserzio- 
ni e da esimersi dalla responsabilità, che addossar voleva il governo ottomano, lasciando con ciò aperto l'adito a maggiori spiegazioni all'arrivo dei riscontri del console.

Speravasi così di aver, almeno per allora, sospesa la produzione di nuove domande, quando una vocale comunicazione della Porta rinovò le inquietudini. Venne imputato il console d'aver contribuito ad agevolare la fuga degli uccisori del gianizzero e senza chiederne il castigo si pretese dalla carica una lettera ostensibile dalla quale risultasse che, colpevole esso dello scampo de' rei, dovesse colla sua responsabilità rinvenirli, farli arrestare e consegnar al mubassir di Smirne. Nessun tentativo fu ammesso per sottrarsi dal rilasciar un documento che preveniva il giudicio sopra un fatto non prima asserto e del quale non si avea alcuna cognizione, ma si accrebbe l'insistenza accompagnata da serie minaccie e dalle più solenni proteste.

Per ben tre volte fu forza di estender la lettera, ed altrettante ostinatamente si ricusò di riceverla perché non era nel sopradetto modo concepita. Ciò nulla ostante sortì con destre maniere di farla accettare quale si assoggetta al numero 10, sebbene non interamente analoga al spiegato desiderio, giacché in essa si annuncia al console, che viene imputato reo senza qualificarlo per tale, gli s'ingionge di procurar l'arresto degli uccisori, anziché di farlo eseguire, e lo si rende alla carica e non ad altri responsabile.

Consegnata la lettera col mezzo del fedele dragomano Mascellini, il quale in grazia d'una malattia sofferta dall'altro dragomano Ralli s'impiegò lodevolmente in tutto il corso di questa spinosa trattativa, trovossi necessario di scrivere nuovamente in modo risoluto al console l'occlusa numero 11, spedita per espresso, onde obbligarlo a discolparsi dalle accuse ed imputazioni che date gli vengono.

Si prevede pur troppo che difficilmente riuscirà di procurar l'arresto dei rei fuggiti, i quali s'erano veramente protetti dalla Russia si tenterà ogni via per sottrarli alle indagini, e che non sarà per produrre delle prove incontrastabili che lo giustifichino appieno, molto più che verso la Porta sono necessarie delle carte turche alle quali soltanto suol ella attenersi. Difatti nella sua lettera al numero 12 non accompagna se non se un'attestazione dei sudditi capitani e solo promette di spedire ulteriori documenti.

Si è per altro il conforto di rilevare dalla lettera stessa che in mezzo alla disgrazia accaduta sia riuscito mediante le cure impiegate a salvare dalle fiamme l'archivio e i depositi della cancelleria. Se però i promessi documenti non sien tali da togliere ogni dubbio ed oscurità sulla sua condotta e non dimostri ad evidenza che si prestò in passato all'esecuzione dei comandi della carica riguardo ai sudditi malviventi e vagabondi, sarà forza di chiamarlo a questa parte per render conto del suo operato, locché potrebbe essere indispensabile anche per mitigare l'irritamento della Porta contro di lui, abbastanza palesato colle serie comunicazioni dal reis effendi fatte vocalmente giunger. 
A questo passo si sarebbe immediatamente condotta la carica, ma oltre che era di equità e di giustizia il lasciar campo al console di procurarsi dei documenti relativi alla sua condotta, importava sommamente ai pubblici riguardi il poterli ottenere, e poi la sostituzione in sua vece non era per allora possibile, attese le preliminari disposizioni che si rendevano indispensabili. Malagevole il rinvenire un soggetto che in tanta delicatezza di circostanze potesse al caso meritare la fiducia della carica nel fungere interinalmente le di lui veci, conveniva occuparcene in prevenzione. Non si trascurarono pertanto i più scrupolosi esami anche in questa parte e risultò che il giovano di lingua Giovanni Battista Navon unisse tutte le prerogative a tal uopo, avendo dati in passato continui saggi della sua capacità ed onestà del suo carattere. All'annunzio che gliene fu fatto si manifestò esso timoroso per la gravità dell'incarico, ma guidato nondimeno da principi di onore e desideroso di procurarsi con un particolare servigio nuovi gradi di merito, si dimostrò rassegnato e pronto ad obbedire alle disposizioni della carica, sicché dovendosi richiamar il console sarà esso a quella parte spedito con commissioni analoghe alle circostanze ed all'importante ufficio che deve sostenere.

Impossibili prevedersi quali esser possano le successive direzioni della Porta nel molestissimo argomento di cui si tratta, qualunque congiettura sopra ciò sarebbe azzardata in tanta contradizione ed oscurità di rapporti.

Dopo essersi sparsa la voce che alcuni stranieri ministri abbiano avvanzate delle pretese di risarcimento per i danni sofferti dai rispettivi sudditi, che in complesso si fanno ascendere a somme molto esagerate, sempre però difficili a quidditarsi, si rimarca nel governo una particolare attività per conoscere ne' modi più cauti ed avveduti ond'ebbe origine il fatale emergente, al quale fine furono spediti a Smirne commissari per formare rigoroso processo.

Stanca la Porta di ulteriormente soffrire le continue violenze e l'indisciplina non solo dei veneti vagabondi e malviventi, ma eziando de' capitani e loro equipaggi, che formarono il soggetto di molti dispacci di me Foscari, in oggi non dissimula più il suo rissentimento. Egli è invero mortificante che mentre dal governo s'imputano i veneti di essere la causa primaria dell'incendio di Smirne, risulti dalle lettere ai numeri 2 e 12 del console che in mezzo all'orrore di sì funesto spettacolo ed alla universale desolazione, gli equipaggi de' veneti legni siano scesi a terra per derubare ciò che le fiamme devoratrici non avean consumato e che i loro capitani li abbiano accolti ai rispettivi bordi cogli effetti derubbati.

Da questa infame condotta desumono motivo vari ministri del corpo diplomatico di aggravare sempre più i veneti presso la Porta, onde indurla a scacciarli indistintamente da tutte le strade dell'Impero ottomano, e far cessare in tal guisa le loro competenza nel commercio. Non lascerà la carica d'impegnare tutti i mezzi che possono es- 
sere in sua facoltà per comprovare solennemente al governo che la Repubblica non ebbe mai intenzione né vuole assolutamente proteggere nessuno di que' sudditi individui che in qualsivoglia modo osassero di turbare la tranquillità pubblica. Le più risolute misure però non saranno giammai abbastanza efficaci per rimediare radicalmente a tanti disordini quando non concorra la sovrana autorità dell'eccellentissimo Senato con addattate providenze valevoli ad impedire l'eccessiva emigrazione dallo stato de' sudditi dalmatini, e de' gre$\mathrm{ci}$, in particolare allettati da straniera protezione, ed a frenare l'indisciplina che regna nella marina mercantile, oggetto bene spesso di scandalo e disonore presso l'estere nazioni.

Guidata la condotta tenutaci sin'ora dall'ardentissimo desiderio di allontanar possibilmente le conseguenti molestie che sembrano minacciate per l'infausta ed imbarazzante sopravenienza, servirà ad alleviare l'amarezza che ne prova l'animo cittadino la clemente approvazione dell'eccellentissimo Senato, dalla sapienza del quale implora la carica le istruzioni opportune per ben dirigere gli ulteriori suoi passi.

\section{0}

Dalle annesse carte fattemi giungere dal capitan passà rileveranno vostre Eccellenze una nuova incomoda sopravvenienza. Risulta dalle medesime che trovandosi certo Sadick, padrone di barca ottomana ancorato col proprio legno al Prodano presso l'isola del Zante, fu sorpreso da un corsaro maltese; ch'essendo la sua barca fuori del tiro del cannone nemico, due zantiotti per nome Panaiotti Dandonà ed Acromano, ch'erano pure in quel porto, accolsero nelle loro scialuppe i maltesi e cooperarono alla preda della barca ottomana, avente quattro marinai d'equipaggio e varii effetti per il valore di piastre tre mila; che seguito il fatto nella acque custodite dalla Repubblica se ne pretende dalla medesima il risarcimento e che siffatta petizione è scortata alla Porta da due illam del giudice di Modon e del cadì di Patrasso, non che da una memoria dei comandanti civili e militari, uomini di legge ed abitanti di Coron, che tradotti dall'originale turco accompagno a vostra Serenità ai numeri 1, 2, 3.

Chiamato sabbato scorso il fedelissimo dragoman Franchini dal detto capitan passà, nell'atto di consegnargli tali documenti gli disse in tuono fermo e decisivo che non potendo esser predato alcun legno ottomano nell'acque della Repubblica, l'avvenuto era contrario alla buona amicizia colla medesima, non che alle capitolazioni di pace e che dovea il bailo indennizzare prontamente il padrone della barca con modi indegni per opera de' veneti derubato.

Gli feci rispondere però che ignaro del fatto era necessario che mi procurassi dei lumi, onde riconoscere da quali circostanze era accom- 
pagnati, senza di che non si poteva desumere un'assoluta responsabilità del governo per indurlo a compensare i pretesi danni cagionati al suddito ottomano.

Accrebbe allora il suo sdegno soggiungendo al Franchini che non ammettevano alcun dubbio la carte avanzatemi; che il padrone della barca non dovea esser esposto a incertezze né attender troppo il suo giusto compenso, e ch'era dovere della Repubblica l'indennizzarlo, castigare i colpevoli e risarcirsi confiscando i loro beni.

Pericolosa, massime nelle attuali circostanze, una maggior insistenza, ripigliò il dragoman, che non avea facoltà l'ambasciatore di far simili esborsi e che soltano poteva renderne inteso l'eccellentissimo Senato per indi dipender dagli ordini del suddetto.

Riconosciuta giusta la riserva dal comandante ottomano, disse che n'era contento purché scrivessi immediatamente e procurassi d'ottenere pronta risposta, la quale non dubitava che sarebbe corrispondente alla spiegata dimanda.

Vorranno pertanto l'Eccellenze vostre dirigere la mia condotta verso un ministro cotanto fiero ed influente alla Porta, che mediante le usate attenzioni m'era riuscito di rendere meno avverso di prima al pubblico nome, e che da quanto mi risulta ha fatalmente spiegato con maggiore forza il suo sdegno dopo il doloroso emergente di Smirne.

Colla prima occasione informerò di ogni cosa l'eccellentissimo provveditore generale dal mar affinché estenda le sue indagini per lo scoprimento dei colpevoli, non che per le determinazioni che crederà opportune, e sarà poi dalla sapienza pubblica il conoscere se convenisse avanzare qualche rimostranza alla Religione di Malta.

Rilevata con spiacevole senso dalle ossequiate ducali 4 marzo decorso l'inutilità de' maneggi per riannodar la pace colla Reggenza d'Algeri, chiamai i sudditi capitani che qui trovansi e significai ai medesimi le provvidenze emanate dalla Signoria vostra per proteggere e difendere la veneta navigazion mercantile dalle aggressioni dei pirati.

Feci loro intender altresì che dovendo qui giungere in breve una pubblica nave per dar imbarco all'eccellentissimo mio precessore, cercassero perciò di procurarsi qualche carico e di approfittare di tale occasione per verificare la loro partenza.

Sdegnandosi molti di loro, e particolarmente i schiavoni e i bocchesi, nel sentir l'intimazione del dey d'Algeri, e ricercarono il permesso di armar in corso per andar fuori dell'Arcipelago contro tutti i barbareschi; ma nel dar la meritata lode al loro coraggio ed all'attaccamento che mostravano per il proprio principe, gli dissi che questa non era la mente sovrana e che colle suddette deliberazioni era stata bastantemente provveduto alla sicurezza del commercio e della navigazione.

Questo inviato di Russia in nome del nuovo imperatore ha presentato in dono al capitan passà una superba tabacchiera d'oro guarnita di brillanti, avente sul coperchio dipinto in smalto la bandiera turca di sopra e la russa di sotto, e nel fondo le bandiere stesse ma viceversa collocate. 
Giorni sono giunse da Tolone un bastimento francese con bandiera turca avente a bordo varii uffiziali di quella nazione destinati al servigio della Porta per la costruzione del noto bacino, la quale però essendo stata affidata ad un ingegner svedese, come rassegnai ne' precedenti numeri, credesi saranno rimandati alla loro patria.

Improvvisamente, e senza saputa dello stesso internunzio cesareo, è di qui partito per la corte di Vienna il già da molto tempo destinato ambasciatore ottomano e ier s'incamminò pure alla volta di Londra quello che deve risiedere presso il re d'Inghilterra.

Accompagno alla Serenità vostra la nota de' soliti doni distribuiti in occasione del bairam grande a varie figure sulla norma de' miei precessori, implorandone la pubblica approvazione.

Pera di Costantinopoli, li 10 aprile 1797

\section{1}

(Con inserte 7)

Pervenute alla carica le risposte che per le cose rassegnate al numero 19 si attendevano dal console di Smirne, si ha lo sconforto di non esser egli riuscito nel procurarsi i promessi documenti, come scorgesi dall'inserta lettera numero 1, alla quale fu data la risposta che risulta al numero 2. Anzi rileveranno vostre Eccellenze che quei comandanti ottomani ricusano l'istanze de' negozianti e capitani sudditi, che non si volle dar questo al dragomano del console e che a questo convenne valersi del vice console svedese per ottenere la definizione di qualche premuroso affare.

Conosciutasi pertanto la necessità di eseguire quanto erasi in precedenza disposto, si credette di non dover indugiare di più a chiamarlo all'obbedienza della carica, inviando a fungere interinalmente le di lui veci dal giovane di lingua Giovanni Battista Navon.

Estesa già la terminazione che lo destinava a tal uffizio e preparate le segrete commissioni che nella delicata combinazione parvero opportune, si disponeva egli a passare a Smirne, quando un impreveduto ostacolo ne impedì la partenza.

Indispensabile all'uopo un firmano della Porta per esso, ed uno per il regresso dell'attual console, approfittando la carica dello ristabilimento in salute del fedelissimo dragoman Ralli, ne fece per di lui mezzo la ricerca con l'annessa memoria numero 3, concepita nel modo più semplice per poter in seguito, secondo le circostanze, manifestare l'oggetto della domanda, ma con sorpresa fummo aspramente ricusati.

Credendo quindi che il rifiuto derivasse forse dal dubbio che allontanandosi il Franchini da Smirne non sarebbe più per portarsi al 
ricevimento del bei, fu esibito di lasciarlo colà e si ristrinse la ricerca al solo firmano per il Navon affinché con tale scorta fosse in grado di portarsi ad eseguire le commissioni disegnate, locché pure fu negato dal reis effendi, dicendo che la Porta non voleva determinarsi ad alcuna cosa se prima dai commissari spediti non giungevano le informazioni che attendevasi fra pochi giorni.

Sebbene contraria ai vigenti trattati una tal recredenza, fu forza in quel momento piegarsi per non inasprir l'animo del ministro. Rinovata successivamente la domanda, accompagnata dall'abile dragomano Ralli con tali avvedute e prudenti riflessioni da far conoscere al reis effendi l'inconvenienza della negativa, offrì spontaneamente il ripiego d'indicare soltanto nel memoriale che per oggetti di suo uffizio occorreva al bailo di spedire persona a Smirne, mentre allora sarebbesi forse assecondata la ricerca.

Quantunque il partito non sia adatto ai presenti oggetti, giacché non potrà il Navon portarsi a Smirne che come semplice particolare senza il carattere e le facoltà necessarie, nondimeno credesi partito di prudenza di far presentare dal Ralli la memoria come sopra modificata, per aver così il vantaggio che il Navon medesimo possa a quella parte recarsi e ragguagliare la carica sopra alcuni fatti che tuttora rimangono oscuri.

In seguito si faranno quei tentativi che le circostanze fossero per esigere onde ottenergli il firmano per l'esercizio delle funzioni pro consolari, importante ed esenziale riconoscendosi che que' sudditi non abbiano ad esser lasciati nel presente abbandono finché giungano le sovrane prescrizioni sul grave e delicato argomento.

Soggetto di rammarico per chi ha l'onore di servire al Serenità vostra sono altresì i discorsi tenutisi più volte dal dragomano della Porta al dragomano Mascellini, e ripetuti poscia al Rali senza spiegarsi sulle intenzioni del sovrano intorno all'accaduto di Smirne, consigliò in detti dragomani ad eccitare il bailo a prendere in seria considerazione l'affare, li ricercò se pensavasi al modo di rissarcire i danni cagionati e parlando dei chiesti firmani disse che era innutile un console veneto a Smirne, ciocché sia meglio conoscere l'oggetto dello spediente proposto dal reis effendi di non accordare firmano a persona avente carattere di ancora maggior peso al cenno fatto nel riverente numero 19, che abbia forse in mira la Porta di escludere i veneti da quella scala. Destre, prudenti ed evvasive furono le risposte dei dragomani stessi, lasciando però travedere che quanto a rissarcimenti la Repubblica aveva il dritto di domandarli al governo, giacché l'incendio fu promosso e verificato dai sudditi ottomani e i veneti negozianti colà domiciliati perdettero tutte le loro sostanze.

Qualunque siensi questi cenni, non possono essere nascosti alla pubblica conoscenza in argomento che merita la maggior ponderazione anche per le seguenti circostanze. 
Continui e quasi giornalieri sono gli ordini che emana la Porta contro i franchi i quali trovansi presso che ridotti in istato di prigionia. Con recenti firmani è stato prescritto che nessuno possa portar armi né bastoni ferrati, che le botteghe di caffè sien chiuse all'imbrunir de sera, che in esse non si vendano vini e liguori e che la notte tutti debbano camminar col lume in pena della carcere. La più piccola ed innocente unione di varie persone in un medesimo luogo genera ne' turchi sospetto ed inquietudine, si fa vedere con frequenza nei borghi di Galata, e di Pera, lo stesso capitan passà circondato da numerosa truppa ed è tolta affatto quella poca libertà che dapprima qui si godea.

Dirette in apparenza le sopradette misure contro i franchi in generale, hanno però in vista particolarmente i veneti perché sono in maggior numero e perché col ministro di vostra Serenità si tiene un linguaggio più risoluto che con gli altri. Resa quindi ogni dì più pesante la responsabilità della carica, quantunque tutte impieghi le sue cure onde non siavi tra sudditi chi contravvenga agli ordini del governo, non può non procurare somma angustia, conoscendo l'indocile e fiero carattere dei medesimi.

Anche ne' passati giorni insorta questione tra alcuni schiavoni in Bujukderè, tutto il paese si pose in allarme cosicché dietro i reclami fatti da tutti i ministri qui residenti e dalla Porta, convenne spedire a quella parte il capigì di questo palazzo unitamente ad alcuni individui di loro nazione più capaci di sedare il tumulto, nonché un dragomano onde passar d'intelligenza con quel subassì e far imprigionar diversi dei colpevoli.

Altra convincente prova delle cose enunziate la dessumeranno vostre Eccellenze da una domanda avanzata col mezzo del voivoda di Galata. Sarà presente alla pubblica maturità quanto fu rassegnato al numero 17, allorché dalla Porta venne chiesta la nota dei patentati schiavoni che si volea non dovessero oltrepassare il numero di trenta.

Varie le circostanze di allora in confronto alle presenti, si credette di sottrarsi dal produrre la nota e di dar passo all'affare promettendo di diminuire opportunamente il numero delle patenti, di obbligare i malviventi ad allontanarsi de qui e di abbandonarli in caso di renitenza al governo locale.

Fatalmente però l'avvenuto di Smirne fece rinovare la domanda: si chiesero dal voivoda i nomi non solo de' schiavoni ma di tutti i patentati indistintamente e lasciò travvedere che forse la Porta sarà per condursi a passi rigorosi verso di loro, come pure riguardo a quegli veneti che muniti non sono di patenti.

In sì molesta e difficile circostanza le sapientissime pubbliche massime e le capitolazioni di pace potean sole porgere una sicura guida nelle istantanee misure che conveniva prendere.

Fatto studio sulle medesime, da molte sovrane deliberazioni risultò senza equivoco la pubblica volontà che i sudditi che non hanno af- 
fari da trattar in queste parti, e che rendonsi molesti ed inquieti con una irregolare condotta, debbano essere abbandonati al loro destino.

Procurando con destrità pertanto di diferire possibilmente la presentazione della chiesta nota, affine di non lasciar ad un tratto in balìa della violenza ottomana molti sudditi forse innocenti, fu creduto di dover estendere le tre inserte carte numeri 4, 5, 6 .

Consiste la prima in un avviso che, approfittando della tipografia francese, si riputò di por a stampa per maggior intelligenza. In esso viene ingiunto ai sudditi non appartenenti agli equipaggi di mercantili navigli e che non hanno aperta casa di commercio, di disporsi a sortire quanto prima dagli stati del Gran Signore, e mentre si offrono i possibili mezzi per agevolare il loro ripatrio si minacciano gl'inobbedienti di abbandonarli al governo.

La seconda è una lettera circolare spedita a tutti i consoli affinché i sudditi non compresi nelle suddette classi, che trovansi nelle rispettive scale, siano obbligati a restituirsi alla patria, in pena di rimaner privi della pubblica protezione, prescrivendosi inoltre le discipline da osservarsi per quelli che dai loro affari fossero costretti a farvi qualche temporaneo soggiorno e per contenere nella dovuta subordinazione gl'individui addetti alle veneta marina mercantile.

Contiene infine la terza un analogo promemoria presentato alla Porta, nell'estender il quale si ebbe in vista di far conoscere l'esecuzione delle massime di vostra Serenità di manifestare la lealtà e rettitudine delle pubbliche intenzioni, di sottrarsi dal pericolo di una preventiva impetuosa risoluzione del governo, diminuendone il presente senso d'irritamento, e di conservare le prerogative che o per diritto o per consuetudine ebbero sempre tutti i ministri di proteggere que' sudditi che con la loro condotta non se ne rendano immeritevoli.

Dietro queste disposizioni non si lascerà d'impiegare eziando la più scrupolosa vigilanza e circospezione nel concedere le patenti, così esigendo il pubblico riposo e l'interesse nazionale. Ad oggetto poi di accelerare la partenza di quelli che si daranno in nota all'uffizio di questa segreteria, qualora mancassero loro le opportunità per restituirsi alla patria, ed il troppo esteso numero de' medesimi non potendo esser contenuto dal pubblico legno rendesse indispensabile di noleggiare un apposito bastimento, vi si presterà la carica, come praticò in altra occasione il precessor cavalier Zulian di gloriosa ricordanza.

Delicato per i suoi gelosi rapporti e difficile l'argomento, ci lusinghiamo che possano esser approvate le tenute direzioni prese come le sole che la violenza delle circostanze poteva permetter. Ma per assicurare maggiormente in avvenire l'effetto di tante cure e sollecitudini, sarebbe opportuno che l'eccellentissimo Senato discendesse ad avvalorarle con la sua autorità ed estendesse poi ulteriori provvidenze nella Dalmazia e nell'isole tutte del Levante per difficultare ai sudditi di portarsi nelle terre ottomane senz'una espressa necessità, rilasciando alle pubbliche rappresentanze, e a tutti i consoli 
che non sono dipendenti da questa carica, quegli ordini che riputerà convenienti, perché nella concessioni di patenti e passaporti sia posta in uso la maggior riserva.

Con tali mezzi evvi motivo di sperar diminuito il numero eccessivo de' veneti che a torme lasciano la patria per passar in questa capitale, e in altri luoghi dell'ottomano dominio, e privando delle loro braccia il natio suolo, preferiscono di venir a compromettere i riguardi del proprio principe, a turbar la pubblica tranquillità in alieno stato ed a porre in continuo pericolo che ha l'onor di servire vostra Serenità in questa legazione.

P.S. Esteso già e pronto il presente ossequioso dispaccio, giunge la lettera segnata 17 corrente del console in Smirne con vari documenti annessi sopra i quali non permettendo l'angustia del tempo di entrar in dettaglio, viene ogni cosa accompagnata in copia al numero 7 affinché la sapienza dell'eccellentissimo Senato possa darvi il conveniente peso.

Rileverà la Serenità vostra della relazione inserta in detta lettera al numero 6, e scritta dai consoli al comandante Caraosmanoglù, che i rei dell'omicidio del gianizzero, da cui ebbe origine l'incendio, furono richiesti a tutti i consoli e che quei musselim e mollà vengono formalmente accusati di colpevole condotta.

Nel difender le pubbliche ragioni, un qualche vantaggio ritrar potrebbesi da questa solenne e generale incolpazione, ma per fatalità ne viene considerabilmente diminuita la forza delle cose che soggiungono il console nella sua lettera e gli altri nella correlazione

Risulta nelle stesse che accusati i schiavoni, zantiotti e ceffaloniotti di aver approfittato dell'incendio per isforzare i magazzini e rapir le sostanze di que' miseri abitanti, si vollero visitare i bastimenti ch'erano in porto e, cominciando dai veneti, trovarono nella cassa di un capitano degli effetti rubati, cosa in vero mortificante, venendo così sempre più compromessi i pubblici riguardi. Irritato sommamente il sopradetto comandante per siffatta scoperta, sospese le visite onde attende l'arrivo dei commissari della Porta, uno de' quali accennando il console che sia colà giunto; fra poco si sapranno le determinazioni prese in tale proposito. Qualora però il capitano suddetto, o qualunque altro che si riconoscesse reo di furto in così grave circostanza, venisse dalla Porta richiesto, non potrà la carica dispensarsi dall'acconsentire alla domanda per non accrescere l'irritamento e per allontanar ogni dubbio che sottrar vogliansi i colpevoli al meritato castigo.

Pera di Costantinopoli, li 25 aprile 1797 


\section{2}

(Con una inserta)

Avendo reso conto all'eccellentissimo Senato col dispaccio numero 20 della preda fatta al Prodano dai maltesi di una barca ottomana, il cui padrone è protetto dal capitan passà, sono ora in grado di rassegnare la precisa somma a cui ammonta il chiesto risarcimento.

Portatosi il detto padrone a questa casa di vostra Serenità scortato dal comandante della flottiglia che nella stagione invernale incrociava le acque dell'Arcipelago, e che deve tra poco ritornarvi, riassunse la storia del fatto, come risulta dagli accompagnati illam. Indi, parlando del valor della barca e degl'effetti che vi erano caricati, delle spese di viaggio per qui trasferirsi, della sua permanenza e dei danni sofferti per esser rimasto privo dell'unico mezzo che avea per procacciarsi la sussistenza, spiegò la sua pretesa di piastre sei mila, compresa la metà per gli effetti rapiti.

Affronte della inflessibilità turca, mi riuscì di fargli conoscere la trascendenza di tale domanda cosicché, dopo molta difficoltà, si restrinse alla perentoria somma di piastre quattro mila, sulle quali attenderò i pubblici comandi.

Non debbo però lasciar di riflettere che, appoggiato il detto ottomano dal capitan passà, come lo prova anche l'averlo fatto accompagnare dal succitato comandante, può meritar riflesso di non indisporlo, molto più che non vi sono fondate ragioni per combattere un fatto accaduto nella acque custodite della Repubblica e colla cooperazione di sudditi veneti.

Con sommo mio rammarico poi l'indicato comandante parlando meco della sostenuta spedizione, si espresse in modo alquanto mortificante su qualche pubblico legno incontrato in cammino. Disse che avea rimarcato molto disordine ed insubordinazione negli equipaggi, che i navigli erano sprovveduti di forze e nella stato in cui trovavasi un tempo la marina ottomana, facendomi di più una pittura poco vantaggiosa della fortezza del Zante e del suo presidio. Conchiuse che nella sua futura crociera vi si porterà forse di nuovo e che potrebbe anche inoltrarsi maggiormente, lasciando in tal guisa sospettare che sia per giungere sino a Corfù.

A prima occasione renderò di ciò avvertito l'eccellentissimo provveditore generale da mar, certo che il conosciuto di lui zelo non lascierà di prestarsi possibilmente nelle circostanze in cui trovasi, affinché i pubblici legni e le piazze del Levante dieno una idea più corrispondente alla pubblica dignità.

$\mathrm{Ne}^{\prime}$ passati giorni questo incaricato di Danimarca, colla mediazione del capitan passà, conchiuse in nome del suo sovrano la pace col Cantone di Tripoli. Vuolsi che le condizioni non saranno palesi se non dopo la ratifica del trattato, ma è probabile che alcune delle stesse non si rileveranno precisamente neppur in seguito, perché d'ordina- 
rio non amasi di pubblicare per intero simili negoziazioni, sostenendosi sempre di averle condotte a termine senza esborsi. Si sa per altro di certo che il trattato è molto più vantaggioso di quello proposto dal dey alla persona incaricata di negoziare direttamente.

Si pretende che il capitan passà s'ingerisca in questi maneggi per soddisfare l'amor proprio e la sua vanità, ed anche per procurarsi dei vantaggi poiché, sebbene sia fornito di grandissime rendite e la grazia del sovrano gli presenti continui fonti di richezze, nondimeno per la somma sua generosità sia aggravato di considerabili debiti, ed è perciò credibile che da questo ministro danese gli verrà fatto qualche significante regalo.

Sopra questo proposito, così esigendoli il dovere di offizio, devo far parole di nuovo all'eccellentissimo Senato dell'ingrato emergente di Algeri. Ben lontano dallo scostarmi dalle pubbliche massime, e venerando le sovrane deliberazioni che le confermano, col prescrivere alla sommessa mia obbedienza di uniformarvisi, come feci, se una si può dire ministeriale communicazione non mi sforzasse, continuerei ad usare il più profondo silenzio in tal argomento.

Parlando il capitan passà in aria di compiacenza della suddetta pace con i suoi medico, tesoriere e dragomano, quest'ultimo gli fece riflettere che di poca utilità può risultare siffatta negoziazione e di non molta gloria per il mediatore attesa la debolezza del Cantone di Tripoli, ma che riuscirebbe vantaggiosissimo il maneggio per pacificare gli algerini colla Repubblica di Venezia.

A tale proposta mostrando il piacere di riannodar la pace, ricercò ad essi se avessero alcun rapporto con qualche persona della legazione veneta per dar principio alla trattativa, su di che avendo risposto il medico che conosceva il dragomano Ralli, e che assumevasi di parlargliene, il capitan passà lo incaricò di concertare col suo dragomano il modo di condurre l'affare e di tenerne discorso sollecitamente. Di fatti non passò molto che ne venne fatta apertura al Ralli stesso, dimostrandogli l'utilità che poteva derivarne alla Repubblica, perché il suddetto ministro avrebbe certamente conclusa la pace a condizioni molto migliori di quelle proposte al Bellato dal bey d'Algeri, e aggiunse ancora che questa erangli note perché il bey medesimo avea scritto al suo padrone di non immischiarsi nella quistione e che, quantunque il capitan passà lo stimasse assai per essere anch'esso passà a tre code, s'impegnerebbe di scrivergli in modo da ridurlo alla ragione, facendolo desistere da domande tanto esorbitanti.

Istrutto da me il fedelissimo Ralli delle pubbliche determinazioni, cercò con destrità di esimersi da un tale discorso, ma colla maggior insistenza venne eccitato replicatamente a comunicarmelo. Lo incaricai quindi di rispondere che avea mostrato molto gradimento per interesse ed impegno che a favor dei veneti palesava il capitan passà, che quanto alla rottura col Cantone d'Algeri l'eccellentissimo Senato, gravemente offeso per l'infondate ed ingiuste ricerche 
del bey, avea preso delle risolute determinazioni, dalle quali riconoscevo non esservi più luogo a negoziazioni, ma che per altro desideravo ch'egli si mantenesse nella buona disposizione onde sperimentarne gli effetti in qualche altro affare.

Eseguì il Ralli colla solita esattezza la mia commissione, ma gli fu in seguito significato che era dispiaciuto il capitan passà di rilevare che il bailo facesse poco conto de' suoi buoni uffizi e che non gli stessero molto a cuore gl'interessi della sua nazione, a favor della quale esso ministro voleva impegnarsi, che se per le deliberazioni del governo credevo di non essere neppur autorizzato a parlarne potevo chiederne l'assenso e che anzi mi si eccitava espressamente a scriverne al Senato, nella ferma persuasione che sarebbero gradite alla Repubblica tali manifestazioni e che, ad esempio della Svezia e della Danimarca, ne farebbe il conveniente uso.

Vedendo però che la costante repulsa di non voler nemmeno partecipare le fattemi aperture potevano generar dispiacere ed indisporre vieppiù l'animo del capitan passà, feci ripondere che avrei di tutto informato l'eccellentissimo Senato. Nel soddisfar per altro a questo involontario impegno, non so dispensarmi dall'aggiungere alcune considerazioni dettate dal vivo interesse che nutro per le pubbliche cose.

Le prescrizioni di vostra Serenità di dover tenere possibilmente lontana ogni occasione nella quale la Porta potesse immischiarsi nelle vertenze coi Cantoni di Barbaria, manifesta chiaramente quella maturità e prudenza ch'io non lascierò giammai di venerare. Ma nella circostanza presente non si tratta che la Porta voglia ingerirvisi, o sia ricercata d'intervenirvi, mentre è il solo capitan passà che non come ministro della Porta, ma come semplice agente delle reggenze affricane, cerca d'interessarsi, a brama d'impiegare la propria influenza presso le medesime per dar termine al fastidioso emergente.

Quando vostre Eccellenze m'ingiunsero d'evitar la di lui mediazione, mi comandarono insieme che non avessi a disgustarlo, ma se ciò era sperabile prima che mi facesse giungere appositamente l'indicato uffizio, rendesi ora per me affatto impossibile senza l'appoggio di precise pubbliche istruzioni.

Il motivo che, per quanto apparisce dalla scrittura che trasmessa mi venne, sembra aver allontanato l'eccellentissimo Magistrato dei V savi dall'offrir il consiglio di valersi della interposizion della Porta sul dubbio che non fosse per prestarsi coll'impegno all'importante oggetto opportuno, e considerando in quel caso giustamente svantaggiosa una semplice intimazione, non esiste per certo nello stato presente dell'affare. Se il capitan passà spontaneamente ricerca d'essere impiegato nella negoziazione è indubitato che si adoprerà colla maggior efficacia e farà uso di tutta la sua influenza, e che parlerà al bey d'Algeri un linguaggio atto a produrre il contemplato effetto, mentre non riuscendo verrebbe ad esser compromesso il suo decoro e la sua dignità. 
E siccome non ignora esso le pretese del bey medesimo e protesta di voler assolutamente interessarsi per vantaggio dei veneti, così si dee credere che offrirà al caso delle proposizioni molto ragionevoli.

Adempito al dover mio nel render conto di ogni cosa all'eccellentissimo Senato, sarà dalla sua sapienza il dare quel peso che meritar possono le fattemi comunicazioni, prescrivendo alla mia obbedienza come avrò in seguito a dirigermi.

Versando i precedenti dispacci sopra materie troppo gravi, mi sono riservato di produrre la nota delli dispendi che incontrai in effettivo danaro e della relativa distribuzione di vari generi per arrestare il capitano ceffaloniotto dalla Porta richiesto per i torbidi promossi dagli schiavoni a Bujukderè; per invigilare alla disciplina de' sudditi in questa scala; per blandire il voivoda di Galata acciò, dovendo necessariamente frapponere qualche indugio per far lo spurgo de' patentati e produrre la chiesta nota, con agisca con soverchia precipitazione verso i veneti; per i corrieri spediti a Smirne e, infine, per coltivare la relazione amichevole col comandante della sopra accennata flottiglia dell'Arcipelago, non solo per esser ben affetto al capitan passà, ma anche perché deve recarsi di nuovo in quell'acque. Mi onoro dunque di assoggettarla nell'inserta dettagliata carta, implorandone la sovrana approvazione.

Rileveranno vostre Eccellenze nella medesima alcuni doni che nell'occasione della partenza, seguita ne' giorni scorsi, dell'ambasciator tripolino, feci in discrete misure presentar allo stesso dal fedelissimo segretario Alberti, il quale nel complimentarlo in mio nome, non lasciò con destre ed insinuanti maniere di mitigare possibilmente il senso d'irritamento ch'avea in addietro dimostrato. Meno aspre del solito furono le di lui espressioni al momento del congedo, ma non saprei dire quanto sia da fidarsene, avendo soltanto il conforto che nel trattar con esso sopra varie incomode ed imbarazzanti domande, mi sortì di servire ai sovrani precetti senza compromettere dal canto mio i pubblici riguardi. La Porta gli fece dono di due legni da corso, l'uno armato di 28 e l'altro di 24 cannoni, benché quest'ultimo sia della portata di 24 c[annoni], ed i loro equipaggi sono quasi per intero composti di gente franca.

A più tranquillo momento presenterò all'eccellentissimo Senato l'interessante tema della scuola turca, dovendo frattanto implorare la permissione di poter qui trattenere il fedelissimo dragomanno Mascellini, il quale destinato a passar in Dalmazia colla ducale 14 marzo 1795, si presta con distinta abilità e con singolar impegno ad istituire i giovani di lingua e s'impegna eziando utilmente, come indicai nel dispaccio del numero 19, in molti affari della legazione, quando se ne affollano nel medesimo tempo in troppo numero e quando particolari circostanze impediscono di valersi degli altri dragomani.

Sempre più considerabili sono i progressi che va facendo nella Romelia il ribelle Pasvanoglù e di giorno in giorno si accresce il numero 
delle sue truppe, allettate a servir sotto le sue insegne dai modi generosi con cui le tratta e dal seducente linguaggio che tiene alle medesime.

Egli è della classe dei giannizzeri; va spargendo di essere estremamente irritato nel veder l'avvilimento di questa truppa in confronto delle altre di recente istituzione, e dice di volerla rimettere al primiero splendore e disciplina. Carca ancora di cattivarsi l'animo de' popoli ne' paesi che occupa sollevandoli dalle presenti gravose imposizioni, lo che tiene sommamente inquieta la Porta la quale per combatterlo non può servirsi dei giannizzeri perché o disertano o abbracciano il partito del ribelle. Si teme qualche insurrezione ad esso favorevole anche in questa capitale e pretendesi che occultamente si facciano strangolare molti giannizzeri. Hachì passà trovasi tuttavia in Andrianopoli, in attenzione de' rinforzi che da questa città a quella parte si avviano, per marciar contro il suddetto unitamente ad altri tre passà.

Pera di Costantinopoli, li 25 aprile 1797

\section{3}

(Con inserte 7)

Accusato dal console in Smirne con la lettera accompagnata nel poscritto dell'antecedente numero 21 il capitano Spiritoff d'essere partito da quel porto nel giorno stesso che gli si dovea far la visita, e contro i di lui ordini, benché in angustia di tempo non si lasciò tosto di dar il conveniente peso alla circostanza. Quand'anche fosse stato innocente, il capitano poteva si sospettare che avesse asportato degli effetti di considerabil valore, e così diveniva necessario di prendere verso di lui qualche misura.

Poco dopo d'esser qui giunto, depositata in questa segreteria la regia patente e inalborato il russo paviglione per portarsi in Mar Nero, si credette di render inteso delle notizie pervenute alla carica questo ministro di Russia con la memoria numero 1, onde non si recasse ad offesa d'avergli taciuta la condotta del capitano se qualche reclamo fosse avanzato contro il medesimo.

Pareva naturale che dietro a ciò il ministro si dovesse determinare a far praticar una rigorosa visita al bastimento, ovvero a sospenderne la partenza finché giungessero alla Porta le relazioni de' suoi commissari, ma invece il giorno susseguente al ricevimento della suddetta memoria spedì alla carica il suo cancelliere per avvertirla che sarebbero levate allo Spiritoff le russe spedizioni e che perciò si mandasse una figura pubblica al bordo per esaminarlo.

Inutili tutte le ragioni per persuadere il cancelliere a differir un tal passo sinché fosse meglio conosciuta la cosa; disse che gli ordini ricevuti non ammettevano dilazione. Fu quindi con esso concertato che si 
porterebbe in sua compagnia questo fedelissimo segretario Alberti per fare congiuntamente una visita al bastimento, praticata la quale, e non essendosi trovata cosa che al capitano o all'equipaggio non appartenesse, gli furono non ostante levate immediatamente le spedizioni.

Qualche giorno dopo l'inviato di Russia rispose alla memoria colla nota numero 2 e contemporaneamente produsse il capitano Spiritoff la supplica numero 3, con varie attestazioni annesse per giustificar la propria condotta.

Quantunque però dalle siffatte perquisizioni risultasse la di lui onestà, essendo incerto se prima avesse trafugati degli effetti, non si riputò conveniente di dargli alcuna risposta, anche per attendere se giungevano le informazioni dei commissari della Porta.

In mancanza di queste e di altri riscontri, passati alcuni giorni si scrisse al console l'unita lettera numero 4 per fargli conoscere come vengano rivocate in dubbio le di lui asserzioni e per rimproverarlo di non aver con più sollecitudine partecipata l'irregolare partenza del suddetto capitano. Siccome poi dalla susseguente lettera numero 5 del console stesso, in data 25 aprile, non si poté rimaner soddisfatti della sua diligenza, perché ripetendo le cose di già soggettate nulla dice di ciò ch'era importante di ragguagliare, non parve opportuno di frappor ulterior remora nello spedire a quella parte il giovane di lingua Giovanni Battista Navon, con le commissioni che risultano al numero 6 .

Non gli si è dato per ora il carattere di pro console e si è creduto di lasciar scorrere ancora qualche giorno prima d'insistere sull'ottenimento del firmano di viaggio per qui richiamar il console, onde vedere se la Porta manifesta alfine d'aver ricevuto dai suoi commissari le relazioni, giacché il reis effendi si è riservato a quel momento di rispondere alla domanda.

Dalle segrete indagini però che sul disgustoso argomento non lascia di fare la carica, risulta che siano di già pervenute. Ma con sommo rincrescimento dai riflessibili cenni contenuti nel poscritto dell'ultima lettera del console e dell'affettato silenzio del governo, evvi pur troppo motivo di dedurre essere vero quanto si asserisce, cioè che si mediti di far responsabili i veneti di quanto è accaduto a Smirne e che non somministrando le informazioni base sufficiente per sostenere tale pretesa siansi dalla Porta occultate e ne abbia chieste dell'altre corrispondenti alle sue mire.

Inopportuno presentemente qualunque passo; si reputa di dover soltanto rimaner in osservazione e affine poi di allentare ogni motivo di doglianza si tiene vigile la carica sulla condotta de' sudditi capitani e loro equipaggi, come apparisce dall'inserto avviso numero 7, non che dagli altri sudditi tutti, e dispone frattanto ciò ch'è necessario per l'allontanamento da questa capitale di que' che vorranno approfittare della fattagli offerta.

Pera di Costantinopoli, li 10 maggio 1797 


\section{4}

(Con inserte 7)

Alcuni documenti pervenutimi da Corfù sopra la pretesa dell'ottomano Sadich per i danni cagionatigli dal corsaro maltese, mentre mi porgono motivo di far nuovo cenno a vostra Signoria dell'affare, mi offrono qualche base per tentar di sottrarsi dal chiesto indennizzamento. Per una di quelle combinazioni, che sono inevitabili quando manca una regolata corsa postale, la lettera scritta dall'eccellentissimo provveditor generale da mar sino dagli otto dello scorso gennaro mi giunse dopo le indicazioni fatte dal capitan passà, ed espresse nei riverenti miei numeri 20 e 22 .

Dall'annesso numero 1 apparisce dunque che impetiti dal suddito ottomano li due zantiotti al cadì d'Arcadia, emanò da questi una sentenza il loro favore e che mercé le vigili cure del nobiluomo governator di nave Orio fu astretto il maltese a restituire al proprietario la barca predata.

Rappresentata la cosa al capitan passà per fargli conoscere la mala fede del suo protetto nel nascondere fatti tanto essenziali, cercai d'indurlo a non accordargli ulteriore appoggio. Ma anzi che persuadersi sostenne che in confronto della sentenza del cadilaggio suddetto era molto più attendibile l'arzmahzar dei comandanti, uomini di legge e abitanti di Corone; che la carta esibita era priva di legalità ottomana; che mancando la quietanza della parte era un semplice asserto di restituzion della barca e che anche senza la cooperazione dei sudditi della Repubblica, doveva la medesima esserne responsabile per essere succeduto il fatto in acque da essa custodite.

Credetti perciò di fargli significare che, possedendosi l'originale dell'offerta carta, me lo avrei fatto spedire; che avrei fatto constare ancora con qualche documento la restituzione della barca; che una informazione da chi lontano dal luogo poteva essere stato sorpreso e deluso era per certo meno valida di un giudizio pronunziato da un tribunale conoscitore dell'accaduto e che, per ultimo, non essendo seguito l'avvenimento sotto il tiro di cannone di qualche piazza, o di pubblici legni, i quali però trattenuti talvolta da venti contrari, da bassi fondi e da altri ostacoli, non possono trovarsi in ogni dove, non era la Repubblica tenuta a risponderne.

Continuando non di meno l'insistenza del capitan passà, che replicava sempre con forza maggiore le medesime ragioni, ed avendo conchiuso l'incaricato dragomano che fata nota ogni cosa all'eccellentissimo Senato se ne attenderebbero i comandi, si persuase alfine di non tenere su di ciò un ulterior discorso se non dopo i pubblici riscontri.

In aspettazione di questi, or che palesi sono alla sovrana autorità i rapporti tutti dell'affare, mi parve frattanto opportuno di scrivere all'eccellentissima primaria carica da mar la lettera numero 2, chiedendo i documenti di sopra citati. 
Vostra Serenità, con l'ossequiosa ducale 30 aprile dell'anno decorso, mi prescrisse di produrre dopo il mio qui arrivo un robusto promemoria alla Porta affinché con un firmano imperiale fosse obbligato il pascià di Valona a restituire al suddito capitan Dabinovich taleri due mila a forza estorti al capitano Murovich, comandante un di lui trabaccolo.

Prevenuto dalla diligenza dell'eccellentissimo mio precessore, il quale ottenne il firmano che unisco al numero 3, mentre ne attendevo l'effetto, giunse alla Porta con apposito messo la relazione numero 4 del prefato passà. Sostiene in essa che sono calunniose e false le rappresentazioni sulle quali emanò il firmano e che creditore verso il Dabinovich di taleri 4752, li due mila non per violenza ma volontariamente ed a conto del suo credito furono esborsati dal Murovich, avendo questi promesso in oltre che il Dabinovich si porterebbe personalmente alla Valona per liquidar i conti. Propone quindi il passà stesso che approfittando il detto capitano dell'opportunità di qualche pubblico legno, giacché spesso se ne trovano in quell'acque, si porti colà, ovvero a Durazzo, per far esaminare di nuovo l'affare, ricerca che sia garante il ministro di vostre Eccellenze qualora non pagasse, e non venendo accolte le di lui proposizioni domanda la permissione di assicurarsi il suo rimborso sequestrando qualche veneto naviglio che si recasse in quel porto.

Preso da sdegno il reis effendi, pretendendo che con falsi rapporti gli fosse stato carpito il firmano, nel manifestare il suo irritamento al fedelissimo dragoman Ralli, gl'ingiunse di consegnarmi la suddetta relazione e di significarmi che senza indugio dovessi scrivere all'eccellentissimo generale di Corfù affinché obbligasse il Dabinovich a portarsi alla Vallona per definire la pendenza e soddisfare il passà, altrimenti prenderebbe delle misure analoghe alla giusta ricerca.

Pervenutami nel frattempo la lettera dell'eccellentissimo Magistrato dei $\mathrm{V}$ savi alla mercanzia nel proposito, trovai fortunatamente tra le altre carte copia di una quietanza rilasciata nel 1787 dal procuratore del passà al capitan Dabinovich per la società commerciale che tra loro sussisteva e su cui appoggiano ora le pretese del passà stesso, il quale nega maliziosamente la facoltà data al procuratore di rilasciarla, e sostiene di essere stato leso nella ripartizione degli utili sociali perché essendoci un terzo compagno avea una maggior somma dal capitano ricevuta.

Ad onta di tali eccezioni e di non esser autentica siffatta quietanza, invece di accordar la chiesta lettera al provveditor generale, mi parve conveniente di produrre il promemoria che mi onoro di assoggettare al numero 5 . Con questo ho cercato di far conoscere la mala fede dell'ottomano comandante, l'ingiustizia delle sue domande e la violenza usata al Marovich contro tutte le leggi di ospitalità ed umanità. E come la carta non è originale, e per il complesso delle cose presenti le circostanze sono sfavorevoli, non ho riputato d'insistere 
sulla inutilità di nuovi esami in un affare che sembra non ammettere dubbio, né sulla esecuzione del firmano per il deposito almeno di taleri due mila fino a questione decisa, non solo per esser ciò contrario alle leggi turche, ma anche perché la Porta dona uno spiegato favore al passà, mercé la protezione che gode di autorevoli soggetti, e in particolare della stessi reis effendi.

Evitato in tal guisa un maggior irritamento nel ministero, rassegno ogni cosa all'eccellentissimo Senato, affinché colla sua sapienza devenir possa a quelle deliberazioni che troverà più conferenti all'oggetto. Non ho lasciato altresì d'informare l'eccellentissimo Magistrato de' V savi alla mercanzia e il nobiluomo provveditor estraordinario di Cattaro, acciocché, se fosse a quella parte il Dabinovich, sia avvertito di tenersi pronto ai pubblici comandi nel caso che la Serenità vostra credesse di ordinare ch'esso, o un suo procuratore, si portasse a Durazzo con i documenti necessari per rimettere la questione all'esame di giudici arbitri i quali avessero a pronunziare una definitiva sentenza.

Ad onta del costituto annotato dal suddito capitano Giacomo Tiozzo, in grazia delle zelanti cure del benemerito residente in Napoli, su di che ne feci cenno a vostre Eccellenze nel mio numero 7, non per anco comparve in questo porto. La renitenza che mostra di assoggettarsi al giudizio a fronte di tante significazioni e ricerche replicatamente fatte dalla Porta, irrita al sommo grado questo ministero ottomano. Il reis effendi ne mostrò la maggior amarezza al Ralli, ricercando che gli fosse mantenuta la fatta promessa e tacciando di debolezza chi rappresenta la Repubblica a questa corte, perché non si faceva obbedire; soggiunse che il governo s'indurrebbe alfine a spedire apposita persona a Venezia per conoscere se ivi pure fosse eguale l'effetto degli ordini che diconsi rilasciati.

E gli è oltre modo mortificante e dolente per me il rassegnar a vostre Eccellenze cose cotanto disgusto, ma ci sono spinto dal dovere e dalla necessità che vengano prese delle misure robuste e positive per obbligare assolutamente il detto capitano a qui trasferirsi, acciò con un ulterior colpevole ritardo per parte sua non siavi nuovo motivo di compromettere i pubblici riguardi.

Allestendosi attualmente la flotta, che nella prossima campagna servirà sotto il comando del capitan passà, e non dovendo esser ignota a vostra Serenità la forza della medesima, come fu sempre cura de' miei precessori, ho procurato con mezzo segreto di ottenere l'unita nota numero 6 dei legni che la compongono, al qual fine dovetti sborsare al confidente reali 100 .

Essendosi i giorni scorsi portato il Gran Signore alla visita del collegio dei paggi del serraglio esistente in Pera, tutti i dragomani dei stranieri ministeri, giusto il consueto, furono a complimentarlo, regalandogli cadauno un desert. Forzato di uniformarmi all'altrui condotta, incontrai la spesa di reali 406 nell'acquistarlo e nell'adornarlo di adatti rinfreschi. 
Finalmente il vice console di vostre Eccellenze ai Dardanelli mi fece giungere la solita polizza, che accompagno al numero 7, dei dispendi straordinari da lui incontrati per pubblico servizio ascendenti alla somma di reali 566, implorando sì di questa che delle due sopraccennate partite la sovrana approvazione.

Un tragico avvenimento è di recente accaduto in Canea per opera di quegli abitanti ottomani. Mossi eglino dall'estrema avversione che nutrono per quei raià greci, presero la crudele risoluzione di volerli uccidere. Non valsero le esortazioni di que' comandanti per contenere gli ammutinati, rappresentando ai medesimi che il partito preso era contario al loro interesse perché tutti traevano la sussistenza dalle terre coltivate dai greci. Abbandonati al loro cieco furore ne passarono molti a fil di spada e la strage sarebbe stata maggiore se opportunamente rinforzata quella guarnigione non venivano arrestati i sediziosi.

Sabbato scorso questo inviato estraordinario e ministro plenipotenziario di Russia, riconfermato nella sua missione dal nuovo imperatore, si portò alla visita del primo visir e ieri a quella del sultano, per presentare le sue credenziali, e sì l'una che l'altra furono verificate senza alcuna innovazione di cerimoniale.

Con quel senso di dolore che non può essere separabile da ogni cittadino animato da fervido amor patrio, ricevetti le ducali 17 e 24 marzo e primo aprile decorsi, con le quali vostre Eccellenze degnandosi di accompagnarmi varie inserte riguardanti grave e rattristante argomento che riempie di rammarico l'animo mio. In mezzo a tanta amarezza non può il mio zelo che ammirare la somma sapienza pubblica nelle prese deliberazioni, confidare che dalla medesima emaneranno quelle ulteriori provvidenze che il miglior bene della patria fosse per richiedere, e far un cauto uso dei documenti pervenutimi qualora le circostanze mi vi obbligassero.

Diretti i comuni voti a vedere una volta ristabilita la tanto sospirata generale tranquillità, desidero che vi contribuisca la pace della quale si assicurano segnati i preliminari tra sua maestà l'imperatore e la Repubblica Francese, benché se ne ignorino le condizioni, avendo questa mattina il dragomano della Porta comunicato tale notizia alle rispettive legazioni, e conseguentemente anco al ministro di vostra Serenità per mezzo del fedelissimo dragoman Ralli.

Pera di Costantinopoli, li 10 maggio 1797 


\section{5}

(Con inserte 18)

Chiamati alla Porta la mattina dei 16 corrente con solenne formalità tutti i dragomani dei ministri stranieri residenti a questa corte, fu introdotto dal reis effendi il fedelissimo Ralli in preferenza agli altri e gli venne consegnata una carta dicendogli che in essa contenevasi le deliberazioni che frattanto preso avea il governo sull'ingrato affare di Smirne. Aggiunse poi che per commissione della Porta gli significava che non avendo il veneto console fatto arrestare gli uccisori del gianizzero, come risultava dalla lettera ostensibile responsiva a quella che gli ordinò di procurarne l'arresto, si richiedevano al bailo che non si desisterebbe mai da questa posizione sinché non sieno consegnati e che si voleva l'immediata destituzione del console stesso. In seguito si fecero entrare uno per volta gli altri dragomani tutti, che poco dopo sortirono dalla camera di udienza cadauno con una carta in mano.

Fatto presente alla carica dal dragomano suddetto il colloquio avuto, e rassegando la carta inserta al numero 1, si pensò subito di dar esecuzione a quanto erasi dapprima divisato, chiamando cioè a questa parte il console Franchini senza pronunziare sull'assoluta di lui deposizione e dando il carattere di proconsole al giovane di lingua Navon, al qual fine si estesero le annesse carte numeri 2, 3, 4 .

Si fece poscia rappresentare alla Porta che manifestato già dal bailo il suo desiderio di qui richiamarlo per giustificarsi sulle accuse appostegli, il ritardo nel verificarlo era proceduto dalla mancanza dei firmani che non si vollero accordare se non giungevano prima le risposte dei commissari e che si avrebbe bensì sospeso il console dalle sue funzioni, ma che non si poteva decidere sulla assoluta sua dimissione se prima non si ricevevano gli ordini dell'eccellentissimo Senato, che sulla nomina del bailo lo rivestì di tal carico.

Molto insisté il reis effendi perché fosse assolutamente dimesso sostituendo altro console in attualità, ma alla fine, sulle destre e prudenti considerazioni fattegli dall'abile dragomano Ralli, sortì di ottenere i firmani necessari senza che vi fossero in essi espressioni offendenti i pubblici riguardi.

E poiché restava ancora pendente l'effetto delle doglianze avanzate da questo ministro di Russia contro il console, essendosi creduto con la memoria accompagnata a vostra Serenità nell'ossequioso numero 19 di tenersi per allora di riserva sinché giungevano maggiori chiarimenti sopra la vera origine e la protezione che godevano gli uccisori del gianizzero, si decise di cogliere l'opportunità della sospensione del console per offrir al suddetto ministro la dovuta soddisfazione coll'indicargli intanto ch'era stato richiamato e che si attendevano poi sul suo destino avvenire i comandi che la Serenità vostra avesse creduto di emanare. 
Dovevasi necessariamente differir un tal passo per la mancanza dei firmani, indispensabili onde chiamarlo a questa parte, e per vedere eziando qual piega prendesse l'affare dal canto della Porta. Speravasi dapprima che avrebbe forse potuto il console ottenere un qualche incontrastabile documento comprovante il fatto da esso asserito, che gli omicidi fossero coperti dalla russa protezione. Ma il complesso delle cose rassegnate dal medesimo, le vocali comunicazioni della Porta sopra quanto l'inviato di Russia le avea fatto significare e l'inserta lettera numero 5 del giovane di lingua Navon, fecero svanire affatto ogni dubbio sopra tal punto, risultando dalla lettera stessa che il vice console di Russia abbia ottenuto un illam che smentisce assolutamente quanto il veneto console aveva asserito.

Perduta pertanto ogni lusinga, mentre il ministro di vostre Eccellenze era per fare tenere all'inviato di Russia una nota corrispondente alla domanda avanzata, all'improvviso nel giorno 22 corrente fu questi a visitarlo e gli comunicò confidenzialmente le risposte pervenutigli dalla sua corte dietro a quanto avea egli rappresentato, scorgendosi dalle stesse ch'erasi ingiunto al ministro costì residente d'interrompere ogni corrispondenza con la Repubblica e di allontanarsi finché veniva data la conveniente riparazione.

Travagliata la patria da tante luttuose vicende, grave senso recò questa, quanto ingrata altrettanto inattesa sopravvenienza. Animati però dal desiderio vivissimo di far cessare con la maggior prontezza possibile una tale molestia all'eccellentissimo Senato, si credette importante d'impiegare i mezzi più efficaci per conseguire l'intento e per corrispondere all'amicizia dimostrata dall'inviato di Russia, e sempre coltivate dai ministri di vostra Serenità, prevenendo così le sovrane commissioni.

Si trovò dunque opportuno di regolare la nota già preparata per l'inviato stesso in conformità della nuova circostanza, e di aggiungervi un promemoria alla Porta, che si accompagnò al ministro medesimo, come apparisce dall'inserte numeri 6 e 7, onde far spiccare senza equivoco che la Repubblica, e quelli che hanno l'onor di rappresentarle alle corti guidate da princìpi di rettitudine, sono sempre pronti a disapprovare solennemente dalle false asserzioni, quando la verità si rende loro palese, facendosi studio di coltivare la buona intelligenza colle amiche potenze.

Manifestata avendo il ministro la più piena soddisfazione per questa sincera e leale condotta, non si è senza lusinga che possa essere soffocato nel suo nascere ogni mal inteso, e che non verrà disapprovata da vostre Eccellenze una direzione tendente ad un oggetto cotanto interessante.

In seguito di ciò si prese in accurato esame la nota fatta tenere dalla Porta al ministro di vostra Serenità, simile a quelle consegnate ai dragomani dell'altre nazioni, come risulta dall'inserta numero 8, giacché riuscì di averne un esemplare. 
Tre punti essenzialissimi contiene la medesima. S'imputano i baili di non essersi prestati come dovevano alle insinuazioni fatte loro di tempo in tempo per contenere in disciplina i sudditi e perché venisse negata la protezione ai malviventi; si attribuisce ai soli veneti la causa del fatale disastro di Smirne e della desolazione di quella città; per ultimo, con offese delle capitolazioni, non si vuole accordare agli ambasciatori di Venezia quella parità di diritti e rappresentative che osservasi cogli altri stranieri ministri, giacché dalle espressioni della nota par che si restringa a lasciar che siano protetti soltanto i capitani ed equipaggi della mercantile marina quando i sudditi siano delle provincie in essa nominate.

Se però si riflette alle voci sparsesi, ed avvalorate dagli oscuri sensi più volte pronunziati dal dragomano della Porta, di voler escludere affatto i veneti dal dominio ottomano, o per lo meno dalla scala di Smirne, di procedere contro la persona del console direttamente anche nell'attualità del suo carico e di chiedere alla Repubblica un compenso per i danni cagionati dall'incendio, si riconoscerà che le conseguenze di questo molesto affare erano con ragione da temersi molto più gravi di quelle che apparivano sin ora dalla carta summenzionata.

Soggetto di mature considerazioni si fa perciò se convenisse di rimaner in silenzio, ovvero se formar si dovesse qualche risposta alla carta medesima. Imperciocché se per l'una parte il silenzio poteva esser interpretato come un tacito assenso alla fatte accuse e alle misure prese, risultava per l'altra molto più spinosa e difficile la difesa, mentre giustificar se stessi non si poteva non aggravar il governo locale, irritando vieppiù la Porta, che lo avea confermato, volendolo in tal modo far credere innocente, e mancavano poi documenti attendibili dai turchi per smentire le imputazioni date ai veneti, la di cui condotta pur troppo non è esente da una giusta censura.

Ma siccome dal tenore della nota, e dal modo solenne con cui fu comunicata a tutte le nazioni, si può dedurre che il governo abbia con essa voluto giustificare in faccia gli stranieri la sua direzione, e preparandosi forse una discolpa al caso che da qualche potenza venissero ulteriormente spinte le domande di compenso già avanzate, così parve partito di prudenza il conformare l'inserta memoria numero 9, nella quale accennando dei semplici fatti e cercando che con l'unico appoggio delle stesse espressioni della Porta, senz'aggiunta di riflessi, rimangano dissipate le imputazioni date ai baili, combattute le conseguenze che pretendesi di dedurre dall'origine del fatto, e non alterato quanto fu proposto nell'altra memoria 21 aprile decorso, apresi una via per allontanar il tentativo coltivato dal governo di costituire responsabile la Repubblica dell'accaduto, e per sostenere i propri diritti dietro le sovrane istruzioni dell'eccellentissimo Senato, che s'implorano precise per farne uso qualora un maggiore sviluppo dell'affare obbligasse a nuove rimostranze. 
Importante poi essendo di non differire più oltre la partenza da qui de' sudditi non contemplati nella memoria stessa, si è pubblicato il proclama che si rassegna al numero 10. In esso vengono eccitati a prodursi all'offizio di questa segreteria entro il perentorio termine di giorni tre tutti quelli che non eransi per anco dati in nota, in pena di essere abbandonati al governo; si prescrive che nello spazio di giorno otto debbano presentarsi per prender imbarco tutti coloro che si sono già prodotti alla segreteria, o che vi si producessero durante il nuovo periodo di tempo; si annullano le vecchie patenti, affinché quei che ne sono coperti credendo d'esser protetti non siano renitenti nell'obbedire agli ordini della carica, ed infine si offre di munire d'un nuovo documento que' tali che avendo legittime cause fossero costretti a soggiornar per qualche tempo in questa capitale.

Oltre a ciò, essendo incerto il numero de' sudditi che saranno per chieder imbarco, si distribuisce intanto que' che si presentano sui veneti bastimenti mercantili esistenti in questo porto, per indi secondo la quantità farli passare sul pubblico legno che si attende, e se non potesse tutti contenerli, noleggiarne uno apposito, sempre con viste di minorar il dispendio, che le circostanze forzano d'incontrare.

Per ultimo si fece un'aggiunta di circolare numero 11 a tutti i consoli, con cui si accompagna in copia l'articolo della carta ministeriale ottomana che può loro servire di lume, obbligandoli colla propria responsabilità a non concedere la pubblica protezione se non se alle oneste dite mercantili nelle rispettive scale stabilite.

Nessuna commissione si è rilasciata sulla domanda della Porta ch'esige in ora gli uccisori del gianizzero dal ministro di vostre Eccellenze, perché non si ebbero ancor i contrassegni necessari per agevolare il loro rinvenimento. Questi erano stati chiesti al console in Smirne, come la Serenità vostra lo avrà rimarcato nella lettera scritta al medesimo li 30 marzo decorso, ma non avendo fatta alcuna indicazione se ne rinnovò la commissione al proconsole Navon, come apparisce dall'inserta numero 3, e quando giungano saranno resi noti ai consoli tutti con apposite circolari, se ne farà precorrere la notizia agli eccellentissimi provveditori generali in Levante, e Dalmazia e Albania, e si parteciperanno pure all'eccellentissimo Senato, affinché rilasciar possa gli ordini convenienti.

Sarebbe confortante per chi ha l'onor di servir vostra Serenità in questo malagevole incarico, che la cauta e prudente condotta tenuta sin ora, da cui si può dedurre la meno sfavorevole piega che ha preso l'affare, potesse porger bastante lusinga di vederlo terminato senza ulteriori molestie, ma fatalmente le cose di sopra esposte, e quelle rassegnate nei precedenti numeri, non possono per anche lasciar tranquilli abbastanza, che per opera degli altrui interessi non ne derivino delle fastidiose conseguenze. Con quel medesimo zelo però, che seguì di guida sino a questo punto, non si lascerà di star vigili 
sopra tutto ciò che potesse risultare in appresso, onde regolarsi in modo da salvare possibilmente i pubblici riguardi.

E frattanto, perché niente manchi a lume dell'eccellentissimo Senato sopra questo interessante argomento, nell'accompagnare alla sovrana sua autorità le lettere del console in Smirne, numeri 12, 13, 14,15 e 16, si reputa in dover la carica di assoggettare che non avendo avanzato la Porta verun reclamo contro il veneto capitano Spiritoff, ed essendosi la dita mercantile Paolo Thoron e compagni, che gode molto credito in questa piazza, fatta per esso lui garante con il costituto di piaggeria numero 17, venne partecipata ogni cosa, con l'unita nota numero 18, all'inviato di Russia, dal quale gli furono restituite le imperiali spedizioni.

Pera di Costantinopoli, li 24 maggio 1797

\section{6}

(Senza inserte)

Nell'attuale politico prospetto degli affari di Europa, tenendo sempre presenti le pubbliche sapienti massime, non rimaneva all'ossequio mio alcun dubbio che la somma prudenza dell'eccellentissimo Senato fosse per allontanarsi dall'adottato sistema d'imparziale neutralità. Posero nondimeno una più sicura base alle mie direzioni le venerate ducali 8 e 22 aprile decorso le quali, con gli accompagnati species facti, mi somministrarono una piena conoscenza dei fatti e delle circostanze.

Il calunnioso proclama, dalla perfidia e malignità de' nemici della Repubblica attribuito all'eccellentissimo procurator estraordinario Battagia, si vide qui stampato in alcuni fogli stranieri, e quantunque il modo in cui è concepito e le mal misurate sue espressioni dovessero far dubitare della sua autenticità, ciò non ostante la Porta vi prestò qualche fede, e questo ambasciatore di Francia in particolare me ne ricercò delle delucidazioni.

Mi convenne però fare un cauto e prudente uso delle cognizioni ritratte dai sopraddetti species facti, del pubblico manifesto 12 aprile e del successivo proclama in data 17 del mese stesso, verso la Porta per mezzo del fedelissimo dragomano Ralli, e direttamente poi con questo ministro della Repubblica Francese, essendomi riuscito colla scorta di tali documenti e di aggiustate riflessioni, di cancellare possibilmente i dubbi introdottisi e di comprovare nelle avvertenti misure prese da vostre Eccellenze la rettitudine delle pubbliche direzioni.

Ciò nondimeno, benché dissipate in qualche modo le sinistre impressioni, non può l'animo mio non provare la più tormentosa angustia, insciente come sono, qual esser possa l'esito delle sapientissime deliberazioni sovrane, per opera dei male intenzionati, e quindi im- 
paziente attendo ulteriori riscontri capaci di ridonare la calma all'agitato mio spirito.

In questi giorni si è sparsa la voce che il capitan pascià non sia per passare quest'anno in Arcipelago con la flotta, e una tal voce acquista un maggior fondamento perché, dietro gli avvisi pervenuti al governo d'esservi nell'acque suddette dei corsari maltesi, si fece tosto partire una flottiglia composta da una nave, una crovetta, tre fregate, sei crovettine e tre chirlangis.

Alfine però di uniformarmi a quanto praticano gli altri ministri qui residenti, non credo di poter dispensarmi dalla solita visita al capitan pascià, e molto più in grazia della presente posizione dei pubblici affari, e dell'avvenimento di Smirne, per rilevare con tal incontro ciò che ne pensa e coltivare in esso delle disposizioni non sfavorevoli ai riguardi dell'eccellentissimo Senato.

Si dice cha alfine sia riuscito alla Porta di far cessare interamente le insurrezioni del ribelle Pasvandoglù medianti gli onori e le distinzioni di cui fu ricolmato, rimettendolo nella grazia del sultano, e che si sia ritirato pacificamente a Vidino.

Spirato nel giorno 11 corrente il secondo trimestre della mia legazione, ho rilasciato al venerato nome di vostra Serenità la solita cambiale di reali [****], che verrà presentata dal mio procuratore conte Giuseppe Mangili, supplicando per la sua accettazione e pagamento in scadenza.

Pera di Costantinopoli, li 24 maggio 1797

\section{7}

(Senza inserte)

Relativamente a quanto fu rassegnato con precedente dispaccio, gl'individui di veneta nazione privi di mezzi per verificare il loro rimpatrio vennero distribuiti sopra i vascelli mercantili ancorati in questo canale, e si rilasciò poi il necessario passaporto a quegli altri tutti che incontrar potevano il viaggio a proprie spese.

Mancanti però i capitani di forze per contenerli, e facendosi lecito contro il divieto di scender a terra, venivano dalle guardie ottomane arrestati dietro i severi ordini dati dalla Porta, sicché irritati i lor compagni, non men che i lor connazionali che formano la maggior parte degli equipaggi de' bastimenti, promovevano delle risse, compromettevano ognor più la carica ed esponevano all'imminente pericolo di qualche sinistro avvenimento.

Imbarazzante una tale circostanza, non avendosi veruna notizia che seguita sia la spedizione della decretata pubblica nave, fu forza di determinarsi al pronto allontanamento dei medesimi individui, in 
grazie pure dei pressanti eccitamenti dati a tal fine dal governo, onde togliere così il soggetto di nuovi disordini. A questo fine si maneggia la carica per formare un contratto di noleggio con que' capitani che offriranno di trasportarli a Corfù per una minor somma, riservandosi di porgerne del risultato i dovuti riscontri con altro corriere. Come poi è necessario che i bastimenti che sortono da questo porto siano muniti di un firmano per il libero passaggio dai Dardanelli, così si sono fatti i passi necessari all'uopo, e si cerca pure di ottenere dal capitan passà, in mancanza di scorte, bujunildì che garantisca il suddetto trasporto dalle molestie degli algerini, locché sarebbesi forse ottenuto se in questi giorni non fosse seguita la solennità del curban bairan, per cui restano sospesi tutti gli affari.

Le lettere del pro console in Smirne Navon in data 2 del corrente dissiparono i dubbi cagionati d'alcune significazioni fatte alla carica da vari mercadanti di questa piazza che tengono corrispondenza con quelli della suddetta città, i quali assicuravano esser emanato un firmano dalla Porta per iscacciare indistintamente tutti i veneti da quella scala, ad eccezione di quei ch'essendo ammogliati volessero cangiar vestito e pagare il karazzo. Riferisce infatti il pro console che interpretati sinistramente gl'imperiali ordini eransi posti in allarme i veneti negozianti colà stabiliti, ma che gli riuscì di tranquillizzarli con la direzione tenuta verso que' comandamenti, ai quali con molta destrità fece comprendere il vero senso dei firmani stessi.

Ciò nonostante, come dalle lettere stesse risulta, che quel voivoda abbia fatte delle difficoltà per riconoscer per veneto il negoziante Matheis colà stabilito per esser egli nato da madre armena, così si può dubitare che il governo sia per condursi a rigorosi esami, onde conoscere se a quelli che considerati furono fin ora come veneti si compete o no la veneta nazionalità; e però non si lascerà di dare al pro console precise commissioni perché tenga istrutta la carica di quanto sopraggiungesse affine di poter far quei passi che le circostanze rendessero indispensabili. Ragguaglia infine il pro console medesimo che in Smirne si praticarono delle severe esecuzioni contro alcuni musulmani imputati di reità nel fatale avvenimento, e che ne furono condannati a morte diciotto, per cui temevasi nuovi tumulti, ma mediante la buona disciplina delle truppe delle truppe del comandante Carasmanoglù tutto procedette senza disordini.

Nella stessa data dei 2 corrente rende conto anche il console Franchini d'aver eseguito le commissioni demandategli onde risursi a questa parte, e che tra due giorni si poneva in viaggio.

Ricreduto avendo la pubblica autorità di condiscendere al ripatrio di me Foscari via di terra, implorato con li dispacci 151, 200 e 237, mi trovo pronto alla partenza fino da marzo, ed in attenzione della di sopra accennata pubblica nave che doveva darmi imbarco.

Invano però io la ho attesa fino a questo momento, e vane pure risultano l'estese indagini onde rilevare se esistesse nell'Arcipelago. Un 
tanto sorprendente ritardo, ch'espone l'erario ad un inutile aggravio e a delle rovinose conseguenze non meno la particolar mia persona, che le pubbliche figure addette alla carica, mi avrebbe già indotto ad intraprendere il viaggio in que' modi che mi fossero sembrati li più pronti, sicuri e meno dispendiosi, ma ciò non è nella mia facoltà, dovendo un ministro qualunque dipendere ciecamente dalli voleri di chi lo comanda.

Esaurito quanto dipendeva da me, onde rendere possibilmente sollecito il mio ripatro, non mi rimane che rivolgermi alla pubblica autorità, affinché deciso senza ulterior ritardo il mio destino, mi sia finalmente comesso di riveder la patria e tributare alla stessa li omaggi della pura mia devozione.

Mancante pure io Vendramin di pubblici riscontri sopra i vari importanti argomenti assoggettati con i dispacci posteriori al numero 15, attendo impaziente le implorate istruzioni che dieno una sicura norma alla mia condotta, onde uniformarmi ai pubblici comandi. Guidato dall'ardente desiderio di manifestare il mio patriotismo e di dare non equivoche prove, non lascierò giammai di portarmi con zelo indefesso, con inviolabil fede e con obbedienza cieca ad eseguire tutto ciò che mi verrà imposto, e mi farò sempre gloria di tributare con rassegnato animo tutto me stesso per il miglior bene della mia patria, alla quale professo il più vero e costante attaccamento.

Pera di Costantinopoli, li 10 giugno 1797

\section{8}

\section{Libertà Eguaglianza}

Cittadini Municipalisti.

Colgo l'opportunità che mi offre la gentilezza dell'ambasciator della Repubblica francese, il quale spedisce a codesta parte un corriere, onde presentarmi a voi per la prima volta.

A ciò son chiamato dall'interesse che nutro pel servigio della patria e perché conosco necessario l'informarvi di quanto ho fatto e credo opportuno di fare per un oggetto tanto importante.

Giunte qui appena le notizie della spontanea rinunzia dell'antico governo, ch'era stata al popolo restituita la sovranità, e che voi ne avevate la provvisionale amministrazione, finché esso con le forme democratiche s'elegga i propri magistrati, vidi i numerosi nazionali che qui si trovano, desiderosi di essere istrutti degli avvenimenti. Mi s'indicò che la Porta dava riflesso alli stessi nei tenuti consigli di stato, che si faceano delle ricerche e che si stava in osservazione.

Benché nelle ducali 13 maggio decorso scrittemi dall'antico governo nell'atto di dimettersi non avessi ricevuta commissione di far io 
il primo alcun passo, conobbi non ostante indispensabile, per allontanar dalla Porta ogni dubbio, di comunicarle le notizie ritratte e di soddisfare i voti dei miei concittadini.

Ho già eseguito il mio disegno verso la prima, che si tenne in silenzio sulle mie vocali significazioni; l'eseguirò immediatamente anche coi secondi e giudico insieme essenziale di renderne con circolare intesi i consoli a questa missione subordinati.

Già son certo che dalla vostra saggezza saranno state trasmesse le opportune istruzioni a chi deve, o dovrà, rappresentarvi a questo governo, che ha colla Repubblica tanti rapporti politici, commerciali e confinari, e dove si trova un numeroso corpo diplomatico; ma non ostante mi affretto di parteciparvi le sue osservazioni e direzioni presenti, perché ciò possa servirvi di lume.

Riceverete, cittadini municipalisti, questa mia attenzione come un saggio del mio verace amor per la patria, al cui bene, alla cui gloria ed alla cui libertà sono pronto a sagrificar me stesso, indotto a ciò dall'appassionato natural mio attaccamento, dal vostro esempio e dalla vostra virtù.

Non vi parlo presentemente d'altri affari, poiché il tempo mi manca, ma mi riservo di darvene conto in continuazione ai miei dispacci colla prossima posta di Vienna.

Salute e fratellanza.

Pera di Costantinopoli, li 20 giugno 1797 - anno primo della libertà

\section{9}

(Con inserte 13)

\section{Libertà Eguaglianza}

Cittadini municipalisti.

Gl'interessi della libertà, il bene della patria, a cui servo e che ha tanti delicati rapporti con questa corte, il mio fervore, l'entusiasmo de' miei concittadini mi chiamarono a far molto più di quello che avea disegno di fare, e che vi accennai nel mio precedente dispaccio, poiché non fui più a tempo di attendere le vostre istruzioni.

Seppi per vari riscontri, verificati anche in alcune conferenze tenute con questo ambasciator francese, che la Porta non calcolava le vocali significazioni avanzatele, e ho quindi creduto necessario di confermarle alla stessa colla nota al numero 1.

Veduta in appresso assunta dai miei confratelli la coccarda tricolore stabilita dal proclama 18 maggio passato, pervenuto qui con lettere particolari, conobbi parimenti indispensabile di comunicar la rigenerazion della patria a tutti i stranieri ministri, onde sopra basi 
offiziali potessero dedur il motivo del nuovo segno d'onore de' veneti, sapessero che quello che intanto provisionalmente a questa parte n'è il capo aderisce pienamente alla riforma, la proclama senza riguardo e ne sostiene fedele i diritti.

Così feci colla nota al numero 2 , segnata nel giorno stesso, che nel pubblico palazzo convocai i nazionali per comunicar loro i felici avvenimenti della patria.

Sarebbe impossibile di rappresentarvi con adeguati colori il commovente spettacolo di quella giornata, nella quale tutti i cittadini si dimostrarono a gara degni figli della libertà.

Raccolti tutti all'ora stabilita nella sala, accompagnato io dal cittadino segretario Alberti, ornati ambidue di coccarda tricolore, mi mostrai ai miei fratelli con quella serietà, verità di sentimento che deriva dal più fervido patriottismo, e con una spontanea effusione di cuore dettata dalla persuasione e dal grande avvenimento, partecipai ai miei concittadini la libertà della patria, i diritti che hanno riacquisiti e i doveri che a essi impone il nuovo carattere.

A questo importante annuncio giudicai opportuno di aggiungere la lettura dei più rimarcabili documenti qui giunti col mezzo de' pubblici fogli, la quale fu susseguitata da mille voci festose, da vivi trasporti di giubilo, da proteste di amor patrio e da vicendevoli baci di fratellanza, di cui ne diedi primo l'esempio.

I ripetuti applausi e le più spiegate dimostrazioni di contentezza non si limitarono però al recinto della sala, ma rinovaronsi alla vista del francese ambasciatore, il quale dal suo chiosco vicino alla casa di Venezia esultava alla gioia de' veneti cittadini e faceva eco alla loro voce ripetendone gli evviva.

Trasportato allora dal patriotico entusiasmo, ed affine di manifestare la mia gratitudine allo stesso ambasciatore, col quale era passato di concerto in tutti i passi, e che avea in quel giorno inalberato il paviglione francese in contrassegno dell'interesse che prendeva alla nazionale sovranità, mi portai a visitarlo e fui seguito da tutti i miei confratelli veneti, schiavoni e greci.

Un energico discorso tenuto dal medesimo in quella brillante circostanza rinovò le generali acclamazioni, e al bacio di fratellanza che loro diede si dimostrarono tutti anche in faccia al rappresentante della Repubblica francese degni del luminoso carattere d'uomini liberi.

Conveniente perciò una più marcata manifestazione della mia riconoscenza verso il sopradetto ambasciatore, mi parve opportuno di scrivergli la nota al numero 3 , alla quale colle più piene espressioni rispose nel modo che risulta dall'altra numero 4.

Nel presentarvi questo dettaglio non posso dissimular la mia compiacenza vedendo non solo accolta con entusiasmo dai veneti qui esistenti la riforma del governo, ma applaudita e gradita insieme, oltreché dal ministro francese, anche da quelli di Olanda, Svezia e Danimarca, come rimarcasi nelle note segnate coi numeri 5, 6 e 7. 
Mi risposero anche i ministri di Prussia, di Napoli e di Spagna, e lo rileverete dai numeri 8, 9 e 10, ma questi per altro si tennero riservati, non espiegando alcun sentimento per attender forse le istruzioni dalle loro corti.

Quelli che osservarono fin ora un perfetto silenzio fu la Porta e furono i ministri d'Inghilterra, di Russia e l'internunzio cesareo.

Pur altro la prima non lascia di far rispettare l'onorevole distintivo della coccarda tricolore e, per quanto mi risulta da sicuri riscontri, si tiene in osservazione sopra un avvenimento che, stringendo vieppiù l'amicizia tra la Repubblica e la Francia, teme che a suo danno risvegliar possa l'antica energia della veneta nazione.

Anche l'internunzio cesareo dimostrò evidentemente l'avervi convenienti riguardi e prevenne le risolute mie rimostranze castigando colla prigionia il suo capigì per aver negato l'ingresso nel palazzo di Germania ad un uomo fregiato della coccarda nazionale.

Vi unisco copia al numero 11 della circolare scritta ai consoli subordinati a questa legazione colla quale, partecipando loro il fausto avvenimento, vengono eccitati comunicarlo a tutti i veneti esistenti nelle rispettive scale.

Insieme vedrete qui annessi ai numeri 12 e 13 il discorso da me pronunziato ai miei confratelli e una relazione di quanto qui avvenne fatta da questo illustre general patriota imprimere nella sua stamperia, e sappiate in aggiunta ch'esso degno rappresentante volle compiere la sua opera col dar un pranzo fraterno a quelli della legazione e ad alcuni veneti capitani, al qual due giorni dopo ho anch'io con egual pienezza corrisposto.

Giunto recentemente da Smirne il veneto console Franchini, sto attendendo le necessarie istruzioni sopra tutto il complesso del grave argomento rassegnato coi precedenti dispacci, giacché non ebbi mai sul proposito riscontro alcuno.

Relativamente ai cenni fatti nel numero 27, e secondo gli ultimi avvisi del pro-console Navon, quel governo locale fa delle difficoltà nel permettere la dimora in Smirne degli originari zantiotti e ceffaloniotti, non che ai negozianti che, sebbene non originari veneti, sono però come tali riconosciuti all'ombra di ducali ottenute dall'antico governo, e chiamati perciò ducalisti.

Col venturo corriere vi spedirò un'esatta relazione dello stato di questo affare, da cui risulterà quanto operai sin ora e ciò che reputerei di far in seguito, mancandomi per compierla i riscontri dell'ultima lettera scritta al pro-console suddetto.

Per non distrarvi con minuti dettagli delle gravi cure che presentemente vi occupano, mi faccio un'eguale riserva anche di presentarvi il metodo da me tenuto rapporto agl'individui di veneta nazione che dietro le intelligenze corse con questo governo e per non comprometter i reciproci riguardi, dovetti allontanar di qui, i quali, a tenor di quanto indicai nel mio numero 27, s'imbarcarono sopra due 
trabacoli da me appositamente noleggiati, che nel giorno 13 corrente fecero vela per Corfù.

Salute e fratellanza

Pera di Costantinopoli, li 26 giugno 1797

30

(Con una inserta)

Cittadini municipalisti.

Le voci, non senza fondamento qui sparse, che siano mal sicure le strade per le quali coi corrieri di Francia e di Vienna vi ho trasmessi i miei dispacci dei numeri 28 e 29, mi determinano a farvene una duplicata con apposito giannizzero per la via di Ancona, troppo importando il loro contenuto perché non abbia a giunger sollecitamente a vostra notizia.

Voi vedrete dalli stessi a quali passi sia stato condotto dall'imperiosità delle circostanze e dal mio filiale e sincero attaccamento alla patria e verso questo governo e verso i stranieri ministri e verso i miei concittadini, benché non avente da voi alcuna autorizzazione.

Ma per altro a norma mia, o di chi dovrà sostenere questa spinosa rappresentanza, è necessario, cittadini municipalisti, che voi avanziate quanto prima il vostro giudizio sulle cose operate e le vostre istruzioni su quello che sarà in seguito conveniente di fare.

È per me dolente il dirvi che qui con troppa facilità si confonde la libertà colla licenza, ad onta ch'io mi affatichi per farla esattamente distinguere, e i capitani e i marinai con pretese e dimande stravaganti mi opprimono e mi angustiano indicibilmente a tutte l'ore.

I primi ridotti in uno stato per vero dire compassionevole, poiché sono qui da molto tempo inoperosi nel porto, attesa la guerra d'Algeri, licenziano i marinai, adducendo di non poter reggere il peso, e questi, lasciati in abbandono, pregano, protestano, si lagnano e minacciano ancora, chiedendo sussistenza e pane.

Io cerco di combinare i reciproci riguardi ora colle insinuazioni, ora con qualche atto di rigore, ma il cimento è duro poiché sono troppo opposti gli interessi e sono compromesso.

Per rimediar in qualche maniera al disordine, la necessità mi dettò il consiglio, approvato pienamente da questo ambasciator di Francia, d'invitar con avviso a stampa gl'individui addetti alla marina, e presentemente senza impiego, a darsi in nota all'officio della segreteria onde prender imbarco sopra un bastimento che noleggerà; ma non so quale sarà l'effetto delle misure adottate e se vi possa esser concorso all'offerta, e ve ne renderò conto col prossimo corriere. 
Anche da un altro importante argomento sono non poco imbarazzato. Quando coi miei concittadini, a tenor del proclama 18 maggio decorso, m’adornai della coccarda tricolore, molti individui stranieri ambirono di partecipar al distintivo ed assunsero il segno nazionale.

Quindi dei contrasti con pericoli di risse coi veri cittadini, e delle ragionevoli osservazioni per conto della Porta, a cui apparve un numero prodigioso di veneti contri i presi concerti.

Io ho già fatte le opportune dichiarazioni e promisi, per togliere l'inconveniente, come farò quanto prima, di proclamar a stampa che l'uso della coccarda conviene soltanto ai veneti cittadini patentati e a quelli che appartengono ai vascelli mercantili.

Per esser anzi certo che le patenti non sieno nella nuova dispensa ottenute che da quelli che ne hanno il diritto, farò che questo venga riconosciuto da una scelta deputazione di probi ed onesti individui, ma non per questo sarà interamente regolato l'affare.

Come potrete vedere, cittadini municipalisti, dagli antecedenti miei dispacci, non soffre la Porta che possano qui rimanere senza distinzione i veneti tutti. Nel procelloso momento del fatal successo di Smirne, colle memorie 21 aprile e 23 maggio decorsi, potei ottener appena che rimanessero le persone che sono nelle memorie stesse comprese, e si convenne l'allontanamento dell'altre.

Ora però gl'individui che qui rimasero, trascurando i miei eccitamenti e i soccorsi offerti per restituirli alla patria che hanno abbandonata, reclamano i loro diritti di cittadini e sostengono di poter rimanere benché non riguardati nelle memorie; ma d'altra parte non si può impedire che questo governo non prenda negli stati suoi quelle deliberazioni che crede opportune, particolarmente contro quelli che possono turbare la pubblica tranquillità.

Finché mi giungano le vostre istruzioni, cercherò di salvare in ogni maniera possibile tutti i riguardi, e mi profitterò dell'appoggio che mi dona questo ambasciator francese, di cui non so abbastanza encomiare lo zelo, il vigor, l'energia ed il vero fraterno attaccamento che palesa per gl'interessi dei veneti.

Anche la spedizione di questo corriere è fatta di concerto con lui, ed anzi il plico vi verrà spedito dal console francese in Ancona, al quale lo raccomanda.

Cittadini municipalisti, date un altro segno della veneta riconoscenza alla nazion francese col ricordar il merito di questo sostenitore e difensore della nostra libertà, poiché egli n’è degno per ogni rapporto.

Siate poscia indulgenti sulla mia condotta, giacché forse avrò fatto più di quello che dovea, o avrò forse omesso quant'era necessario di fare; ma vi protesto che per certo non ho mancato per non aver rette intenzioni e perché non senta vivi in me l'amor della patria e l'interesse per il suo servigio.

Salute e fratellanza. 
Pera di Costantinopoli, li 5 luglio 1797 anno primo della libertà italica

P.S.

Vi trasmetto la risposta che mi diede anche questo internunzio cesareo, la quale, sebbene segnata li 25 giugno, mi giunse solo dopo la partenza del corriere dei 26, giacché trovandosi egli a Bujukderè furono mal eseguiti i suoi ordini per farmela avere.

\section{1}

(Senza inserte)

Cittadini municipalisti.

Non voglio lasciar partire il corriere di Vienna senza tentar di darvi per tal mezzo un qualche riscontro e per inoltrarvi insieme la replica del mio numero 30 . Ho nei dì scorsi ricevute le informazioni che attendeva da Smirne e trovo non piccolo motivo di conforto dal lor contenuto. Seguendo quel proconsole le istruzioni da me ricevute di coprire colla veneta protezione quei ducalisti, di cui vi ho fatto cenno nel numero 29, si accinse accortamente all'impresa e furono d'un esito felice coronati i di lui zelanti maneggi.

Gli fu permesso di comprendere nella nota degl'individui, per i quali esso risponde, non solo i ducalisti suddetti, ma tutte anche l'altre oneste persone ch'esistono da molto tempo in quella città e che non hanno mai dato motivo d’alcuna lagnanza.

Egli per altro mi accenna che i ducalisti suddetti, e quelli particolarmente che sono vestiti all'orientale, non sono ancora senza qualche dubbio che la loro tranquillità possa essere in avvenire turbata, e che richiedono per questo d'aver un firmano imperiale che li tolga d’ogni timore.

Ma nel darmi tali rapporti mi significa per altro che ciò non gli sembra bisognoso all'istante, che si può soprassedere e che, se vegga novità che lo renda necessario, me ne darà gli avvisi accompagnati da documenti propri all'oggetto.

Io però non faccio presentemente alcun passo, contento di quanto risulta e da cui veggo in salvo l'interesse e l'onor nazionale.

Que' poveri negozianti sarebbero stati esposti a delle enormi vessazioni col titolo di risarcir il tesoro pubblico dei diritti che non pagarono sin ora; nella loro rovina avrebbero involti i corrispondenti d’ogni paese; questi avrebbero forse dato motivo a querele, sostenendo di esser con essi entrati in relazione d'affari sulla fiducia della veneta protezione che godevano, e poscia ai veneti soltanto si sarebbe negata la prerogativa, da tutte le altre nazioni goduta, di associare a se stessi e di proteggere degl'individui che non ne sono immeritevoli. 
Io non vi faccio tenere le lettere del proconsole da cui ritrassi sì fauste notizie poiché, unite all'altre non ancora spedite, desidero di accompagnarle con incontro più sicuro.

Ho sospeso dopo il numero 25 di trasmetterle inserte, poiché in quella data nulla vi avea di meritevole per essere assoggettato.

Susseguentemente furono d'una importanza maggiore, e sono in particolar modo riflessibili l'ultime, anche per le carte che mi accompagnano, sicché reputo necessario di farvele avere, onde in tutta l'estensione sia da voi conosciuto un argomento che mi pose nella più grave angustia, che irritò oltre modo la Porta contro i veneti dell'isole e della Dalmazia e che compromise il decoro e l'interesse della nazione in generale.

Fu eseguita la pubblicazione a stampa dell'avviso sui patentati e sulle coccarde ed ho scelti gl'individui ai quali ho rimesso il riconoscimento della veneta nazionalità di quelli che si presenteranno.

Mi attendo da questa misura un miglior effetto di quello ritirato fin ora dall'avviso stampato per gl'individui addetti alla marina che sono senza impiego e che in numero riflessibile pretendevano di essere da me giornalmente soccorsi. Intesa però dai medesimi l'offerta di rimandarli alla patria, soli cinque ne comparvero, e starò a vedere chi si produrrà domani, ch'è l'ultimo giorno.

Salute e fratellanza

Pera di Costantinopoli, li 10 luglio 1797 anno primo della libertà

P.S.

Era scritto il presente quando l'ambasciator di Francia, che non cessa di dar in ogni incontro delle prove novelle del suo interesse per noi, pensò che per esser più tranquilli sul sicuro suo recapito era meglio di suggellarlo coll'impronta della sua repubblica e trasmetterlo al ministro francese costì residente. Mi sembra opportuno il consiglio, e pienamente l'adotto, e ve ne rendo poi conto perché sappiate il motivo della mia direzione.

\section{2}

(Con un'inserta)

\section{Libertà Eguaglianza}

Cittadini municipalisti.

Trova conforto il zelo mio patriottico nello scorgere dalla lettera 17 giugno passato del cittadino vice-presidente la fiducia che in me ripone codesto comitato. Rilevo pure dalla medesima essere intenzione della Municipalità, per i motivi indicati e da me compresi, che io 
mi limiti a riferire in forma affatto privata le notizie che possono aver rapporto con il complesso dell'oscura situazione degli affari d'Europa.

Dai precedenti numeri avrete potuto scorgere l'impossibilità di circoscrivermi a tal uffizio. Le circostanze mi obbligarono a dei passi pubblici ed un'eguale condotta debbo per necessità anche in presente.

La natura degli affari esige che con frequenza mi rivolga alla Porta; i consoli delle scale vicine chiedono istruzioni e suffragi; grande si è il numero de' nazionali che qui trovansi, reso maggiore per la stazione da vari mesi di molti legni veneti in questo porto, attesa la guerra col cantone d'Algeri; continui sono perciò i litigi e le contese de' veneti fra loro, cogli ottomani e con individui di altre nazioni; ed il numero de' veneti viene insieme aumentato dai cittadini della varie ex-provincie dell'antica Repubblica che, avendo mostrata la loro adesione al nuovo governo, a me si dirigono per essere assistiti. Anche i passi fatti con intelligenza e persuasione dall'ambasciatore della Repubblica francese, e voluti da particolari circostanze, produssero altresì il bisogno di una maggior attività ed energia. Quindi il bailo, se non sia diversamente istruito o dimesso dalle passate sue funzioni, è costretto ad agire da capo di nazione, e quasi da rettore di provincia, che tale appunto, a differenza dell'altre, può chiamarsi quest'ambasciata.

Ciò a voi significato, perché vi sia nota per intero la mia situazione, passo a darvi conto di quanto può interessarvi sulla condotta della Porta a nostro riguardo.

Dopo che i molti veneti qui esistenti assunsero la coccarda tricolore, le si sono resi più osservabili e ne concepì dell'inquietudine.

Difatti ne' giorni scorsi chiamò il cittadino dragomanno Ralli, e gli fece significare dal reis efendi ch'essendo i sudditi ottomani dell'isole dell'Arcipelago vestiti come gl'isolani veneti, e potendo derivarne della confusione, dovessi ordinare a que' che portano la coccarda, di vestire alla franca o di abbandonarla e che, secondo quanto stabilito in addietro con l'antico governo, e le recenti misure prese, si volevano allontanati tutti gli schiavoni, a riserva di trenta muniti di patente.

Consultato questo ambasciatore francese, trovò conveniente che scrivessi l'unita nota, da esso spontaneamente appoggiata colla sua memoria pur inserta, onde manifestare vieppiù al ministro ottomano il costante suo impegno e fervore per tutto ciò che riguarda la veneta nazione e la premura colla quale sostiene le mie direzioni. Scorgerete dalla prima che, dopo aver allegate le ragioni per non aderire assolutamente a quanto veniva chiesto, conchiude che renderà inteso il governo di ogni cosa, per dipendere dalle sue commissioni, avendo frattanto insinuato ai nazionali di adattarsi in proposito della coccarda ai spiegati desideri.

Feci poi rispondere quanto ai schiavoni che tutti quelli ch'erano a mia notizia furono precedentemente spediti alla patria; che se altri ve ne sono tenutisi sinora nascosti, procurerei di persuaderli di allon- 
tanarsi, ma che non era possibile che ciò seguisse colla voluta sollecitudine. A questo replicò soltanto il reis effendi la necessità dell'allontanamento, ma dopo alcuni giorni si passò alle vie di fatto e dalla guardie turche ne vennero arrestati parecchi. Mi sono trovato perciò in necessità di produrre ier mattina un'altra nota, che pure accompagno, concertata prima coll'ambasciator di Francia. Fu anzi preceduta dai suoi pressanti uffici poiché, avendo dovuto per gli altri affari suoi vedersi col dragomanno della Porta, sostenne col solito impegno le mie ricerche e non sono affatto senza lusinga che la risposta che si riservò il dragomanno di dare quando abbia comunicato la memoria, non sia per essere favorevole ai miei desideri.

Tali sono i passi da me fatti, ma per render meno incerta la mia condotta e verso questi nostri cittadini, e per meglio regolarmi nel rispondere alle frequenti interrogazioni della Porta e degli stranieri ministri, sarebbe opportuno che mi fossero spediti almeno i decreti e proclami più importanti che con le stampe costì si pubblicano.

Dalle direzioni di questo governo e da quanto mi sortì di rilevare sembra essere invalso il timore che con l'uniformità della lingua possano tra i suoi sudditi greci propagarsi facilmente de' principi che parlano alla ragione e s'imprimono nel cuore dell'uomo più idiota $\mathrm{e}$ che, scossi dal senso di dispotismo che li opprime, siano per recargli delle molestie, pretendendosi dippiù che siano pervenute delle notizie poco favorevoli sull'interna tranquillità della Morea.

Sostiensi ancora da persone degne di fede, che circoli fra greci una carta scritta in versi con la quale sono eccitati a scuotere il giogo ottomano e vendicarsi dell'usurpazione del loro paese ed a prendere a tal effetto le risoluzioni più vigorose.

Io non garantirò l'autenticità di questi fatti, ma è certo che la Porta è in grandissima apprensione sullo stato presente delle cose, attesi i finitimi suoi rapporti con la Repubblica, sentendo mal volentieri che sia approdata a Corfù una squadra combinata di legni francesi e veneti, che si sieno pubblicati colà dei proclami pervenuti a questo ministro francese, e da esso gentilmente comunicatimi, atti a risvegliare l'antica energia della greca nazione; che i bocchesi siansi uniti ai montenegrini senz'aver spiegate le loro intenzioni, ed abbiano anche posto qualche legno in corso e che infine la Dalmazia e l'Istria veneta siano occupate dall'armi imperiali.

$\mathrm{Ne}^{\prime}$ passati giorni poi da alcuni qualificati soggetti turchi, aventi delle relazioni coi ministri della Porta, erasi sparsa voce che questo governo coltivasse l'idea di profittare del divulgato smembramento della Repubblica Veneta per muoverle la guerra e spogliarla dell'isole.

Non sembra però ragionevole che abbia potuto concepire un tal disegno. Troppo è palese che questo impero risente la massima debolezza. È lacerato da intestine discordie per l'insubordinazione dei pascià delle provincie. La marina è senza vigore, non rimarcandosi alcuna straordinaria attività nell'arsenale, e non essendo nep- 
pur sortita in quest'anno la gran flotta, alla quale si è sostituita una squadriglia di legni leggeri. Vi dirò in aggiunta che, destinato il ribelle un tempo Alì Passà a distruggere i ladri che infestano i dintorni d'Adrianopoli, venne ad accamparsi colla sua armata vicino alla capitale. Le sue truppe commisero degli eccessi e finalmente, dopo aver molto resistito agli ordini del sultano, si pose in marcia, ma essendosi manifestato un generale malcontentamento contro di lui, la Porta gli ordinò invece di fermarsi ad Adrianopoli, temendo forse che in luogo di combattere i malviventi non andasse ad accrescerne il numero.

Anche Kachy Passà, antico Bey Sarbey di Romelia, le diede dei serii pensieri, attesa l'aperta dissensione degl'individui componenti il divano, alcuni dei quali voleano opprimerlo ed altri salvarlo. Ei però dovette cedere agli sforzi dei suoi nemici e fu esiliato a Stanchiò, benché i suoi talenti, secondo la comune opinione, meritassero un destino migliore.

Neppur tranquillanti per essa furono le notizie che giunsero recentemente dalla Persia, perché quel re Mehemet Kan, ch'era da lei amichevolmente riguardato, e forse in segreto assistito, perì sotto il ferro d'un principe della sua corte subito dopo aver segnata la pace coll'impero di Russia.

Non venendomi fatto alcun cenno sopra i vari argomenti trattati ne' precedenti dispacci diretti all'antico governo, e in particolare sopra quello di Smirne, che per la parte politica non ha ulteriori conseguenze, attenendomi anche al senso delle ricevute commissioni mi riservo ad altro momento d'inviar la promessa continuazione de' documenti relativi all'affare suddetto, come pure di parlare sopra gli altri di questa missione.

Questo signor inviato di Russia fu inalzato ad un ragguardevole posto nell'interno ministero, e si accenna che verrà rimpiazzato dal general Iamara.

Salute e fratellanza.

Pera di Costantinopoli, 7 thermidor, 25 luglio 1797 v.s., anno primo della libertà italiana

\section{3}

Libertà Eguaglianza

Cittadini deputati.

Corrispose alla mia aspettazione l'esito dell'affare degli schiavoni, di cui vi feci cenno nel mio precedente numero spedito per via di Vienna, e del quale ora vi trasmetto la replicata con il corriere di Spagna. 
Prodottosi alla Porta il cittadino dragomanno Ralli per ricevere la risposta alla presentata nota, fu essa affatto coerente alla medesima e solo si chiese che in conformità prender dovessi ogni cura acciò entro il mese di ottobre prossimo si allontanino di qui tutti gli schiavoni, esclusi i trenta patentati.

Comunicata ai medesimi questa risoluzione, l'accolsero con giubilo, vedendo che con sicurezza possono raccogliere i prodotti delle terre che coltivano, e promisero unanimi di verificar la partenza entro il fissato periodo.

L'influenza dell'ambasciator della Repubblica francese ed il nuovo ordine di cose mi fanno sin ora trovar molta facilità nel trattar gli affari con la Porta, a differenza di quando rappresentavo l'antico governo. È quindi ragionevole la lusinga che se la Repubblica nostra sia per prendere una politica consistenza, non sarà come per l'innanzi condannata a rimaner passiva in tutti gli esterni suoi rapporti.

Riguardo però a questa corte, particolarmente interessar deve le cure del governo l'eccedente quantità di veneti che son qui e nell'altre scale ottomane.

Passano essi in questo impero dalla Dalmazia, dall'Albania e dall'isole del Levante. Una parte di essi impiegansi con onore e vantaggio nazionale nel commercio e nella navigazione, quantunque anche tra capitani non manchino de' scorretti individui; altri poi, costretti ad abbandonare la patria per la loro cattiva condotta, hanno per unico scopo di vivere oziosi e vagabondi in alieno dominio; altri pochi si occupano nel lavoro delle terre; molti infine passano nella marina della Russia e, qualificandosi or dell'una or dell'altra nazione, compromettono sovente la buona intelligenza ed amicizia tra la Repubblica e la Porta, come pur troppo lo comprovano infiniti esempi.

Mi credo in dovere di farvi questo cenno affinché a momento opportuno possa la vostra saviezza prendere in considerazione un tal argomento e stabilire sopra sicure basi quali condizioni aver debbano gl'individui dimoranti in alieno dominio per essere durante la loro assenza dalla patria assistiti da quei che avranno a rappresentarvi e per godere dei diritti di cittadino.

Salute e fratellanza.

Pera di Costantinopoli, li 14 termale anno primo della libertà italiana - 1 agosto 1797 s.v. 
[33a]

Libertà Eguaglianza

Cittadini deputati.

Secondo le indicazioni fattemi nella lettera 17 giugno decorso, ho sospeso di scrivere alla Municipalità come avea cominciato, ed ho diretto al cittadino vostro segretario i miei dispacci.

Avendo ricevuto riscontri coll'ultimo corriere anche dalla Municipalità, deduco che non sussistano più i riguardi di prima. Scrivo dunque direttamente alla medesima, segnando il dispaccio col numero successivo a quello dei precedenti onde evitare le confusioni.

Ho per altro spedito il piego sotto privato addrizzo, attese le politiche circostanze in cui ci troviamo con la corte di Vienna, e della quali fui avvertito dalla diligenza del cittadino Gradenigo, ministro plenipotenziario.

A tenore della vostra lettera 8 luglio decorso, feci degl'inviatimi libretti a stampa quell'uso che mi avete commesso, avendone fatto giungere direttamente un esemplare nella mani non solo del dragomano della Porta, ma anche di qualche altro ottomano ministro.

Voglia la nostra buona ventura che possano produrre qualche utile effetto, ma dello stato di questo governo e della sua indolenza politica ho già reso conto ne' miei precedenti numeri.

Salute, rispetto e fratellanza.

Pera di Costantinopoli, 23 termale - Anno primo della libertà italiana (10 agosto 1797 v.s.)

\section{4}

(Con 6 inserte)

Libertà Eguaglianza

Cittadini municipalisti.

Nelle gravi ed incessanti cure in cui sono tutto giorno involto per servire nelle delicate circostanze presenti utilmente alla patria, giunsero opportune a darmi conforto e a rinforzarmi il coraggio le vostre lettere 8 luglio decorso.

Rilevando dalla vostra saggezza approvate le direzioni da me tenute negli affari assoggettati al passato governo, batto con franchezza maggiore la novella carriera poiché sono all'azione determinato da due possenti stimoli, quello cioè del mio patriottismo e l'altro della vostra indulgenza.

Spero, cittadini municipalisti, d'aver incontrati i desideri che mi avete espressi nelle stesse e che possa esser assicurato il godimento 
degli antichi privilegi al ricco convoglio destinato per Alessandria. Conobbi all'oggetto opportuno di presentar una nota alla Porta, ma volli per altro allontanar per fino l'idea che in noi vi fosse il sospetto che, atteso il nuovo sistema di cose, attentar si volesse ai vigenti trattati.

Come avrete veduto dai precedenti miei numeri, io mi sono diretto in maniera con questo governo da fargli vedere che doveano essere necessariamente più stretti i nostri legami, e quindi celai il vero motivo della memoria, che inserta vi accompagno al numero 1, sotto l'apparenza d'esser da voi incaricato di dar sopra ciò le più positive assicurazioni e di chieder a riscontro il più ospitale accoglimento al convoglio suddetto.

Venne la nota gentilmente accolta dal dragomano della Porta, ma non potei ottenere alcuna risposta per trovarsi il reis effendi gravemente ammalato. Non dubito per altro di non averla favorevole, anche per essere stata appoggiata dai buoni offizi di questo ambasciator della Repubblica francese, il quale mi promise anche di usarmi la compiacenza di scrivere al console della sua nazione in Alessandria, onde raccomandargli i nostri interessi. Vidi che voi disegnavate di maneggiarvi allo stesso oggetto con codesto ministro e non posso essermi ingannato seguendo l'esempio.

Ho insieme creduto necessario di far un altro passo, benché non avente da voi alcuna commissione. Mi giunse coi pubblici fogli costì impressi la protesta divulgata sull'ingiusta invasione dell'Istria e della Dalmazia fatta dagli austriaci, e vi rimarcai scritto in fronte: da esser trasmessa a tutti i governi.

Allora io mi consultai che il mio zelo e quanto esigeva il servigio della patria, onde chiamar delicatamente il governo a dar per proprio interesse qualche pensier all'affare e credetti di doverla comunicare formalmente coll'altra nota, che vi accompagno al numero 2 .

La notizia della detta invasione sconcertò assai tutti i nazionali qui esistenti, e in particolare gli schiavoni. Era d'uopo animarli singolarmente colla lusinga che il fermo appoggio della Repubblica francese non sarà inefficace anche in questa importantissima circostanza, e quindi estrassi dalle varie notizie qui pervenute l'annesso bollettino, che pubblicai colle stampe. Riuscii con questo mezzo a calmare, ma non però a togliere i dubbi introdotti e che dall'arti frodolenti degl'inimici della causa della libertà vengono con ogni studio coltivati.

Viene fatto supporre agli schiavoni che sia di concerto colla Repubblica francese l'occupazione della loro patria, e che perciò debbano infallibilmente essere soggetti all'impero di Cesare. Si insinua che il conveniente e puro partito che ad essi resta è di mostrar il loro buon volere e la loro buona volontà verso il nuovo padrone, giacché altrimenti alla giusta vendetta dello stesse esporrebbero le loro sostanze e le loro famiglie. Si consigliano perciò a deponere l'onorevole insegna della coccarda tricolore, che dagli emissari del pregiudizio e del dispotismo si chiama insegna di sovversione e di disordine. 
Io m’oppongo con ogni maniera possibile a queste occulte macchianzioni, a questi raggiri, e sostengo il vacillante coraggio, ma non so predire quanta esser possa la loro fermezza se non si veda o un pronto ritiro degli austriaci, o che si accenda la guerra. Per il bene comune desidero vivamente il primo e, quando fosse necessaria la guerra, qualunque sacrifizio sarà sempre doveroso se si tratti di sostener col decoro nazionale dei diritti che hanno la sanzione dei più solenni trattati e d'un possesso di secoli, onde metter a parte i nostri fratelli delle provincie occupate dai dolci effetti della nostra rigenerazione.

Se sia vero, come si assicura, che questo internunzio cesareo abbia ricevuto l'ordine di non riconoscere questa rappresentanza, non si ponno presagire certo favorevoli disposizioni nell'imperatore di ritornar con noi alla prima amichevole corrispondenza, e temo pur troppo che ciò si confermi anche per le relazioni avute dal ministro plenipotenziario, cittadino Gradenigo.

Non ho, cittadini municipalisti, ricevuto ancora le istruzioni che nella vostra lettera accennate d'avermi spedite, e che si riferivano anche all'argomento di Smirne. Quantunque ignori per quale via me le abbiate trasmesse, nelle attuali circostanze temo che per la varietà dei contrari interessi e per le cose in esse contenute esser non possa innocente la loro mancanza, e me ne rincresce assai.

Mi si rendevano piucché mai essenziali, particolarmente per sapere cosa avevate deciso per prender in esame la condotta del console. Egli a questa parte si trova e l'affare, in quanto riguarda le sue direzioni, sempre più si avviluppa. Non so quello che possiate costì aver stabilito e se forse nell'importanza dell'argomento e nella molteplicità dei documenti che dovranno esser assoggettati ad un maturo riflesso, abbiate conosciuto opportuno di delegarlo ad un'apposita commissione, ma in ogni caso credo essenziale, onde nulla vi manchi ad una fondata determinazione, di non differir più oltre a farvi avere le carte che qui trattenni e ve le trasmetterò col prossimo corriere di Spagna.

Nel tempo stesso vi accompagnerò anche alcune petizioni che mi sono state avanzate a carico della precedente missione, e che tutte, seguendo lo spirito della democratica riforma, che abborrisce gli arcani nelle procedure criminali, ho comunicate al cittadino precessor Foscari.

Vuole giustizia che si sappia l'accusator e l'accusa, e che abbia luogo una legale difesa, e nella mia direzione son certo d'aver il vostro suffragio. Non ho per altro creduto che a me appartenesse il giudizio, come alcuni petizionari chiedeano.

Egli è bailo ancora; voi stessi nelle vostre lettere l'avete riconosciuto per tale. In parità di carattere di pubblico rappresentante non posso senza una speziale commissione aver sopra lui alcun diritto. ̇̀ assurdo che colla facoltà ordinaria possa un successore decider su 
chi lo precedette nelle direzioni tenute nell'amministrazione del carico. Alle sole autorità superiori è riservato un tal diritto e mi sembra che male si apponga chi crede che questo metodo possa soffrir variazione dalle variazioni dei governi.

Il cittadino Foscari disse che non si sorprendeva se da taluno, cogliendo il pretesto del presente cangiamento delle cose, si cerchi di censurare delle direzioni dipendenti da pratiche e consuetudini inveterate, e soggiunse ch'era pronto a sottoporre le sue azioni all'esame il più scrupoloso, nella certezza che, se qualche nemico tenta un'accusa, sarà pronta e retta non meno l'amministrazione d'una imparziale giustizia.

Consultato questo ambasciatore di Francia su tal argomento convenne nell'opinione ed adducendomi il proprio esempio, manifestommi che nelle memorie a lui prodotte contro il suo precessore non prese altra parte che di accompagnarle a Parigi. Io seguo le di lui traccie, le assoggetterò ai vostri riflessi ed intanto, perché vi compiacciate d'approvarlo, vi accompagno inserto l'atto che dovetti segnare per sottrarmi all'insistenza di chi volea che mi arrogassi una facoltà che non ho e che a voi soli conviene.

Forse quest'oggi sarò un po' troppo prolisso, ma debbo trattenervi sopra un altro argomento. Niente meno ci volea della nostra rigenerazione perché, squarciando francamente il velo, ve ne potessi parlare con quella verità ch'è propria delle anime ingenue e amanti della giustizia.

Se i vostri interessi esigono la sussistenza di questa missione, quest'interessi medesimi, la vostra purità e l'onore di chi vi serve, richiedono ch'essa in rapporto economico sia diversamente piantata. Un salario fisso le dava un'apparenza di mediocrità. Una bonificazione di un zecchino ogni tre reali di spesa apriva l'adito ad una utilità, che non riconosceva altra misura che il confronto delle amministrazioni precedenti. Erano divise le spese in tre classi: limitate, soggette a confronto ed estraordinarie, che si esibivano all'approvazione.

Le anticipazioni di denaro prima della partenza da Venezia, le rimesse ordinarie ed estraordinarie in corso di carica, la consegna e spedizione di generi di manifattura, per distribuirsi in doni, erano assorbite per intiero dall'admesse denominazioni. Dal complesso di esse sortiva quel tanto che alla risorsa del cittadino veniva predisposto e tacitamente concesso, oltre ad un credito che risultava al regresso e che dietro ad una resa di conti di pura formalità gli veniva abbonato.

Il prodotto parimenti dei cottimi offriva una rendita nei tempi passati bastante a supplire ai pesi che le erano stati addossati. Fu però la stessa soggetta sempre a maliziose diminuzioni, ed ora quasi interamente estinta per le circostanze attuali del commercio e perché i sudditi ottomani ricusano di pagare il diritto di cottimo. Su di ciò vi farò parola in altro momento. Non vi tacerò neppure che anche nell'elezione formale ai consolati di Smirne, Salonicco e Canea 
erano soliti i baili per una pratica inveterata di profittar delle offerte che dagli aspiranti venivano loro fatte, admettendo alla percezione secondo le quote consuete anco il loro ministero.

Se questi però sono i fonti da cui la missione trae i suoi vantaggi, ella ha d'altro canto degl'indispensabili pesi.

Un copioso numero di persone addette che pesano sulla sua economia con panatiche ed assegnamento; una scuola turca per i giovani di lingua, quattro dei quali, con il loro maestro dragoman Mascellini, sono alloggiati nel pubblico palazzo e spesati interamente dal bailo per non aver casa propria in Pera, con il compenso poi per i soli quattro giovani di reali 12 a testa ogni mese; ingresso e visite al serraglio ed ai suoi principali ministri all'installazione della carica, d'inveterata etichetta e di molto fasto; il maneggio continuato degli affari della nazione non sempre immune da spese, avidi come sono per se stessi gli ottomani ed avvezzi alla prodigalità con cui procedono gli altri governi, e particolarmente i limitrofi.

Io non ho spedito ancora alcuno dei passati trimestri di queste spese, perché sentiva una viva ripugnanza nell'admettere le decorse pratiche oscure, e mi astengo per ora dall'inoltrarvi la cambiale dello scadente terzo trimestre, non avendo avuto riscontro delle due precedenti.

Ma è di fatto innegabile che la missione non può senza mezzi né sussistere né utilmente servire. È opera vostra, cittadini, il riconoscerli ed il prescriverne le misure secondo il carattere ministeriale che dar vorrete a quegli che avrà qui a rappresentarvi.

Ma avvertite che non bastano i lumi che gli offizi del passato governo vi possono somministrare, e nemmeno quelli ch'io stesso potessi inoltrarvi: li primi perché tratti da carte che ritengono li vizi del passato sistema; li secondi perché turbati e illanguiditi dalla frapposizione di tanta distanza.

Per questo mi sono determinato ad inviarvi il cittadino Ferigo Cameratta, ministro economico, il quale, munito di tutti i documenti opportuni, sarà pronto a rendervi esatto conto dei pesi da me sofferti e che tuttavia soffro.

Vi aggiungerà inoltre quelle cognizioni che un'ingenuità professata, propria del suo noto carattere, può solo manifestarvi, e sarà anche per restituirsi al suo posto, allorché il nuovo metodo rendesse qui necessaria la di lui opera.

Ho già licenziata una parte della famiglia, che non serviva che a peso e ad un lustro men necessario, ritenendo soltanto ciò che rendesi indispensabile per sostenere il decoro della nazione.

Già voi saprete a codesta parte con quali commissioni sia partito per Corfù il general Gentili, e come sia stato incaricato dal general Buonaparte di corrispondere a questo ambasciatore. Riconobbe il general Gentili che non può ritrarre quanto abbisogna il mantenimento dell'armata francese dall'isola suddetta, non volendola, dietro gli ordini ricevuti, assoggettar ad alcuna contribuzione. 
Quindi scrisse per aver dall'ambasciatore suffragio di provigioni e di dinaro, e questi noleggiando un veneto bastimento lo fece ieri partire con bandiera ottomana carico di numero 4500 chilò di grano e con numero 28 mila piastre in contanti sotto la direzione del suo aiutante di campo tenente colonello Casterat.

Manca da vari giorni da questa capitale il capitan passà, che si portò a Gemlenich per comandare un taglio di legni da costruzioni, e di là si crede che passerà ai Dardanelli per esaminare una nave che sta fabbricando il veneto costruttore Spadon.

In questo momento mi giunge in iscritto la promessa che m'avea fatta vocalmente il suddetto ambasciatore di Francia di raccomandar il convoglio destinato per Alessandria a quel console della sua nazione.

Ve l'accompagno inserta congiuntamente a quella ch'io gli avea fatta tenere, onde vediate con quanta prontezza si presti questo degno rappresentante del popolo francese ai bisogni dei suoi amici, e come anzi offra di fare più di quanto era stato richiesto coll'avanzar i suoi buoni offizi per l'oggetto suddetto anche a tutti gli agenti francesi stabiliti nell'Asia Minore.

Salute, rispetto e fratellanza.

Pera di Costantinopoli, li 10 agosto 1797 v.s., 23 termale anno primo della libertà italiana

\section{5}

Libertà Eguaglianza

Cittadini municipalisti.

Dopo firmato il numero precedente mi giunse dalla gentilezza dell'ambasciator di Francia la di lui comendatizia diretta al console di Alessandria di quella nazione a favore del convoglio che da Costantinopoli si dirige per quel porto. Vi aggiungo perciò la copia della stessa ed insieme la nota ministeriale con cui mi venne accompagnata, dalle quali rileverete sempre maggiori le testimonianze di amicizia che il suddetto ambasciatore si compiace di manifestare al popolo veneto ed a chi ha l'onore di rappresentarlo.

Rispetto e fratellanza.

Pera di Costantinopoli, li 23 termale anno primo della libertà italiana (10 agosto 1797 v.s.) 


\section{6}

(Con inserte come nella nota acclusa in fogli 35)

\section{Libertà Eguaglianza}

Cittadini municipalisti.

Eseguisco l'impegno che mi sono preso nell'antecedente numero 34 e vi accompagno il seguito dei documenti che avea a questa parte raccolti, relativi all'affare di Smirne e alla condotta del console Franchini, onde nulla manchi alla vostra maturità nelle deliberazioni che sarete per prendere. Dovrò per altro farvi tenere ancora qualche altra cosa. L'ultime lettere dell'attuale pro-console Navon mi trasmisero varie carte, colle quali il cittandino capitan Bronza, esistente in quella scala, appoggia le pretese da lui spiegate contro il Franchini suddetto. Sono le stesse voluminose; mi pervennero in questi ultimi giorni e mancò il tempo di trarne le copie e farle comunicare al Franchini, ma ve le spedirò quanto prima. Il cenno, che ho creduto di farvi nel numero suddetto, del bisogno che forse vi sarà che voi deleghiate una speziale commissione all'esame di quest'affare mi sembra, quanto più ci penso, non affatto inopportuno, e quindi non so trattenermi dal rinovarvelo. Il solo volume delle carte che costì esistono, di quello che presentemente vi trasmetto e dell'altre che sarò parimenti in necessità di accompagnarvi, pare ch'esiga necessariamente questa misura. A chi sia scevro d'altre cure e d'altri pensieri può riuscire soltanto di svogliere con maturità e riflesso un argomento nel quale la direzione del console fu prima involta in delicati rapporti politici, ed indi complicata con ricorsi di altra natura che furono contro di lui avanzati, e che perciò richiede una ponderazione non ordinaria. Non m'imputate a colpa, cittadini municipalisti, questa libertà di parlare: credo di dovervi dire ingenuamente i miei pensieri, e mi rimetto poi alla vostra saggezza.

Colle carte suddette vedrete unite, secondo la mia promessa, anche le petizioni fattemi in aggravio alla precedente missione e la risposta del precessor Foscari formata in una lettera che mi scrisse. Ne vedrete una segnata dal capitan Milinovich posteriormente all'atto da me stesso esteso, ed essa, ch'è una protesta contro qualunque delle spese e danni, che gli risulteranno dalla dilazion del giudizio, mi porge motivo di presentarvi alcune mie riflessioni. Nulla di più conveniente, opportuno, ed anzi necessario e sacro, quanto che i giudici siano determinati ad amministrar una imparziale giustizia, oltreché dai possenti motivi dell'onor, del dovere, anche da quello della propria responsabilità. Io amo questo freno e mi vi assoggetto volentieri; ma il giudice deve aver una norma, è necessaria una legge e, quando questa non esista, de dev'essere responsabile in caso di provata malizia, giustizia appunto vuole che non lo sia se per mancanza di basi certe e di lumi sia stato indotto in errore. Ve lo ripe- 
to, cittadini municipalisti, la carica che sostengo non è una sola ambasciata, ed io posso chiamarmi ambasciator e pretore, ed infatti a ciò corrisponde l'antico nome di bailo. Inoltre le differenze, le liti, le giudicature, i processi, non vertono unicamente tra i nostri concittadini; più spesso anzi corre il dover del giudizio fra essi ed individui d’ogni straniera nazione, e quindi ci sono sempre in mezzo note ministeriali, reclami della Porta, oggetti politici, riguardi che si debbono avere per il ben della patria alle altre potenze.

Se il corso d'una esatta procedura qualche volta richiede che un più maturo esame preceda la deliberazione, la politica non lo vuole, e non è ancor giunto il tempo felice che la politica sia di perfetto accordo con la giustizia. Questa osservazione è spezialmente verificata dal fatto quando debbasi trattare con questa potenza rozza ed ignara dei veri principi del diritto, e che anche nell'affare di Smirne ne diede una prova recente, quando scagliossi contro l'intera veneta nazione perché alcuni individui della stessa avevano commesso un omicidio. La natura parimenti degli affari che qui si trattano, per la maggior parte commercievoli, esige che non venga molto innovato sulle forme, poiché la dilazione nel definirli può apportare delle conseguenze incalcolabili.

Per la via dei compromessi arbitrari si decisero fin ora le controversie. Ora gl'intriganti che si sono a questa parte stabiliti vogliono introdurre il sistema delle appellazioni alle sentenze d’arbitraggio, e vi fanno un protesto, se non ricevete. Qual dovrà essere, cittadini municipalisti, la mia direzione? Voi, che già grandi in fatto di rivoluzione, mostrate e nella condotta e negli scritti d'aver cominciata la vostra carriera là dove gli altri l'hanno finita, saprete conoscere quali regolazioni potessero convenire allo spirito della stessa, sicché cogli oggetti commerciali e politici sieno combinati i riguardi d'una retta amministrazione di giustizia. Attendo in una norma sicura, che vi prego di dare ai miei passi, il felice risultato dei vostri studi e della vostra prudenza.

Debbo accompagnarvi una memoria prodottami dal capitano Francesco Marinovich, a cui vedrete annessi due attestati rilasciatigli dai capitani esistenti in questo porto e in quello di Smirne. Egli chiede o di aver una stabile funzione a questa parte, a seconda dei desideri dei capitani suddetti, o di esser, come lo sprona il suo zelo, impiegato in qualche altra maniera a servir la patria, che adora. Il decider sulla prima parte della sua petizione può solo dipender dal futuro sistema che stabilirete per questa missione, e posso intanto assicurar ingenuamente ch'egli sosterrebbe con tutto impegno ed onore il carico che gli fosse affidato. Giustizia mi chiama a fargli questa testimonianza. Nelle differenze, nei torbidi, nelle contese dei suoi nazionali io mi sono prevalso dell'opera sua, e ne trassi degli utili effetti. Son certo che corrisponderebbe alla vostra aspettazione, anche se, come accenna, vi valeste di lui nella marina, poi- 
ché la comune opinione è in suo vantaggio spiegata, ed è sommo il suo zelo ed il suo patriotismo.

Salute, rispetto e fratellanza.

Bujukderè, 29 termale anno primo della libertà italiana - 16 agosto 1797 v.s.

[Nella replicata la data topica è: Pera di Costantinopoli]

\section{7}

(Senza inserte)

Libertà Eguaglianza

Cittadini municipalisti.

Vi ho nell'antecedente mio numero 34 riferito qual sia stato il senso prodotto negli schiavoni qui esistenti alla nuova dell'invasione fatta dall'armi austriache delle provincie della Dalmazia e dell'Istria. Il loro vacillante coraggio avea bisogno d'appoggi; vi sono accorso con quei mezzi che in mio poter si trovavano, ma per altro m'accorsi, come già vi ho indicato, che soltanto alla nuova o d'un pronto ritiro dell'armi imperiali, o d'una dichiarazione di guerra, poteano risorgere le cadenti loro speranze.

Quest'ultimo corriere di Vienna fu però ben lontano di produrre contali adattati riscontri l'effetto che vivamente desiderava. A me solo mancavano i pieghi, che forse m'avete spediti, ed anco le corrispondenze private; ma questa capitale fu inondata di pubblici fogli e di relazioni tutte concordi per disanimar affatto gli schiavoni suddetti e per gettarmi nei più gravi imbarazzi. La voce è comune che l'invasione austriaca di concerto coi generali francesi, che immancabilmente le dette province saranno unite ai domini imperiali e che quindi debbono gli schiavoni riconoscer l'imperatore per loro sovrano.

Potete immaginarvi l'agitazione degli spiriti e quante ricerche mi furono fatte. Scorsi avidamente i fogli che potei qua e là raccogliere; mi procurai d'ogni parte notizie; volea pure scaturir qualche cosa da contrapporre alla fama che il mio patriottismo volea farmi suppor menzognera; ma non ebbi che nuovi motivi di profondo dolore. Vidi la terra ferma costante nel volersi mantener disgiunta; che vengono i vostri inviti trascurati e non calcolate le conseguenze dell'ingiusta occupazione; che Verona anzi protesta contro il vostro manifesto e non vuol sentire parole d'unione che colla Repubblica cisalpina; che i francesi non sembrano disposti a sostenervi coll'armi, mentre all'incontro l'imperatore fa le sue tranquille disposizioni per sistemar le provincie invase, vi manda governatori e riceve deputazioni che gli presentano giuramento di fedeltà e d'obbedienza. 
Il mio sconforto e la mia desolazione furono tali a questo aspetto di cose da non saper di preciso in quale maniera dirigermi, e quindi alle dimande fattemi dagli schiavoni a chi dovessero rivolgersi, chi riconoscer per capo, di qual bandiera valersi nella navigazione e che manifestassi qual era il loro destino, potei soltanto rispondere che non doveano esser così facili a prestar credenza alle sparse notizie, che i nostri nemici ingrandiscono i mali, che stessero cauti contro l'insidie che loro ci tendono e ch'io non avea ricevuta ancora alcuna istruzione ma che l'attendeva fra poco.

Quanto poi alla ricerca di qual bandiera dovessero i bastimenti servirsi, ebbi un qualche fondamento dalla vostra lettera 8 luglio decorso, giacché feci ad essi comprender che se con lo stemma di San Marco dovea partir di codesta città un ricco convoglio di vascelli mercantili per Alessandria, poteano essi pure far uso del medesimo finché si fosse diversamente stabilito, e che procurerei inoltre di maneggiarmi verso l'ambasciatore di Francia onde li scortasse con un passaporto, ciocché mi venne da lui graziosamente promesso.

La mia critica situazione per altro non è soltanto prodotta dal fermento e dall'agitazione degli schiavoni, e gli abitanti greci dell'isole vi hanno gran parte essi pure. Si è sparsa voce che anche l'isole venete debbano da voi rimaner separate. Si accenna per prova che i francesi soltanto hanno una parte diretta nell'amministrazione del governo e che al Zante siensi fatti prigionieri i consoli russo ed inglese, ciocché non sarebbesi eseguito in un paese neutrale se avessero creduto d'entrarvi soltanto come ausiliari dei veneti. Si aggiunge anche il raziocinio per convalidar la detta opinione. Apparisce ad ognuno che, restando in poter della casa d'Austria la Dalmazia e l'Istria, ove vi hanno boschi di legnami da costruzione, porti capaci e popoli applicati per indole e per bisogno alla navigazione, può in breve tempo alzarsi al grado di rispettabile potenza marittima, e che quindi l'interesse dei francesi esige che uniscano al loro destino come dipartimenti l'isole venete per bilanciare nell'Adriatico la marina imperiale. In oltre si fanno perduti per la Repubblica anche i paesi di Vonizza, Prevesa e Butrintò, i quali si dicono ceduti alla Porta perché si tenga tranquilla sull'eseguito partaggio. Queste voci, che sono nella bocca d'ognuno e che vengono convalidate dai discorsi della maggior parte dei stranieri ministri (la direzione dei quali è in mio riguardo ambigua e sospesa), mi costituiscono, come lo potete ben comprendere, in una situazione estremamente critica e dolorosa. Io non so a quali passi potranno forse condursi gl'individui delle nazioni suddette. Sono ben lontano dall'agitarmi da vani timori, ma mi si parla il linguaggio della insubordinazione e della licenza; molti dei capitani che ritornano dal Mar Nero ritengono patenti straniere, trascurando di riprender le venete; si esige quello che non si dovrebbe; non sono talvolta ai cittadini ministri risparmiate le offese e le minaccie e vi hanno degli argomenti di credere che sieno fomentati dalla malizia altrui per compromettere. 
Da questo prospetto e dai cenni fattivi nel mio numero 36 riguardo agli ostacoli dai quali mi trovo combattuto nell'amministrazione della giustizia criminale e civile, voi scorgerete, cittadini municipalisti, non solo il pericolo, ma l'impossibilità in cui mi trovo di sostener in tale stato di cose quest'ambasciata. Conosco purtroppo anch'io le sommamente critiche e imbarazzanti circostanze vostre, e quindi la difficoltà che incontrar dovete nel determinarvi, ma dall'altro canto come mai può un cittadino rappresentar un governo di cui si pone in dubbio l'esistenza politica? Manifestandomi senza esitanza e senza velo attaccato alla patria, a cui sarò sempre fedele, disprezzando qualunque sagrifizio e incontrando con lieto animo le fatiche, le pene e la responsabilità che seco portano gli avvenimenti attuali, con l'assistenza e veramente patriotica cooperazione del cittadino segretario Francesco Alberti, ho procurato di servire a tutti i riguardi della nazione e di mantener a questa parte la disciplina e il buon ordine. I miei passi per altro furono sempre condotti dalla ferma speranza di ottener il vostro appoggio e, se lo stato degli affari v'impedisce di prestarmelo, viene ad esser compromesso il vostro decoro e il cittadino che vi serve, non solo in faccia al governo ottomano ma insieme presso tutti questi stranieri ministri.

Anche il mio stato economico esige imperiosamente un pronto provedimento. Nell'attual posizion dell'erario vorrei che le private sostanze mi ponessero in grado di poterlo sollevar dai pesi indispensabili a quest'ambasciata; ma, se in addietro col mezzo di questa conciliavasi un riparo alla fortuna dei cittadini, voi dovete comprendere quali esser possano le mie circostanze. Tutto mi fu contrario fin ora per apportarvi un qualche riparo. Ai primordi della carica dovetti sottostare ad estraordinari dispendi; non furono accettate le cambiali dei trimestri passati e quindi il mio procuratore, cittadino Giuseppe Mangili non può prestarmi alcuna assistenza, sicché sono costretto a ricorrere ad espedienti passivi i quali, accrescendo giornalmente le perdite, conducono rapidamente al mio economico annichilimento. A fronte di tutto ciò mi faccio un delicato riguardo di trasmettervi, sebbene scaduta, la cambiale del terzo trimestre, giacché la mano pronta del mio procuratore suddetto può ricevere e tramandarmi quei suffragi che all'evidenza della mia situazione credesse la vostra equità opportuni.

Qui si osserva un gran movimento in questo ministero. Fu fatto ritornare il capitan bassà; si tengono frequenti consigli di stato; fu spedito a Vienna un estraordinario corriere ed essendosi dimesso il reis-effendi, che, come vi accennai, si trovava ammalato, vi fu sostituito il famoso Rassid-effendi, era tersanà-emini, ossia soprintendente all'arsenale, che per la terza volta ascende a tal grado a lui conferito sempre in incontri delicati e spinosi, poiché è grande l'opinione che si ha dei suoi talenti e della sua politica destrità. Per servire agli oggetti della patria egli fece praticare le convenienti offiziosità dal 
cittadino dragomano Ralli, il quale, essendosi anco veduto col dragomano della Porta, ritrasse, in risposta alla nota prodotta rapporto al convoglio destinato in Alessandria, che questo governo ricorda la sua antica amicizia coi veneti, come crede che questi vi corrispondano coll'aver i convenienti riguardi per i soggetti ottomani esistenti in codesta città, tra i quali nominò particolarmente il negoziante Serpos. Avrei desiderato vivamente che il suddetto dragomano si fosse espresso in una maniera più aperta e decisa, ma le nostre stesse circostanze obbligano la Porta ad una riserva, poiché forse non sa ella stessa nelle vicende presenti quali saranno le direzioni a cui verrà dal suo interesse determinata. Non ostante mi fanno vivere tranquillo le raccomandazioni che ho a questa parte ritratte dalla cortesia ed amicizia dell'ambasciator francese, lusingandomi che non meno efficaci saranno state quelle ottenute da voi e che non ci abbia ad esser anche in questo articolo contrario il destino. Attendo con impazienza, cittadini municipalisti, vostri riscontri e scusate se oso indicarvi che vi compiacciate di spedirmeli per tutte le strade che vi si presentassero, anche sotto direzioni particolari, onde non abbia lo sconforto di restarmene ulteriormente privo.

Salute, rispetto e fratellanza.

Pera di Costantinopoli, li 8 fruttifero anno primo della libertà italiana - 25 agosto 1797 v.s.

\section{8}

(Con la semplice nota delle inserte già spedite col corriere di Spagna)

\section{Libertà Eguaglianza}

Cittadini municipalisti.

Mi valgo del corriere di Spagna che parte quest'oggi per avanzarvi la replicata dell'ultimo mio numero speditovi con quella di Vienna. Col presente poi vi aggiungo tutto ciò ch'è pervenuto a mia notizia e che derriva dai discorsi e congetture che si fanno in questa capitale.

La straordinaria attività che rimarcasi nel consiglio di stato, mi assicurò persona degna di fede, che dipende dall'essersi gli ottomani posti in grande agitazione per la condotta dei francesi e per la voce sparsa che i medesimi aver potessero disegni sopra l'isola di Candia. Su quest'ultimo articolo si assicura che la Porta abbia chiesto una formale dichiarazione a questo ambasciatore e ch'egli non abbia esitato a farla col smentire una tale diseminazione.

$\mathrm{Ne}^{\prime}$ passati giorni l'incaricato d'affari d'Inghilterra ebbe una segreta conferenza col nuovo reis-effendi. Si pretende che abbia avuto per oggetto il presente generale prospetto politico d'Europa. Aggiun- 
gesi che il predetto incaricato abbia cercato di mettere in difidenza gli ottomani e di fargli temere dei pericoli qualor si verificasse quanto si sostiene generalmente, che le isole venete esser debbano unite alla Repubblica francese. Non è improbabile ch'egli abbia tenuto un tal discorso, mentre in tal caso la sua nazione vedrebbe assicurato nelle mani dei francesi il commercio generale del Levante, e quello particolare delle uve passe, che gl'inglesi fanno con l'isola di Zante.

Ma qui non si fermano le congetture e i discorsi. Si sostiene che, per tranquillizzare la Porta nell'occupazione della Dalmazia fatta dagl'imperiali, gli saranno ceduti, oltrecché i paesi di Btrintò, Vonizza, Prevesa e tutte le altre adiacenze nel golfo dell'Arta, anche l'Albania veneta, e che venga istigata a prevalersi della circostanza per invaderla con l'armi. Si dedusse che possa il governo abbracciare questo partito dalle commissioni che si vogliono rilasciare ai passà confinanti.

Tutte le secrete indagini praticate furono inutili per iscoprire se possono meritar credenza questi fatti. Io dunque non me ne faccio garante, ma è però certo che tali discorsi sono molto riflessibili in momenti critici come i presenti e nella capitale di un governo che potrebbe realizzarne il piano.

Alcuni accreditati soggetti turchi che hanno delle strette relazioni con il ministero ottomano sostengono ancora che, rendendosi vieppiù osservabili le mire della nazion francese per estendere da ogni parte i suoi rapporti, venga fatta conoscere alla Porta la utilità di accedere ad una nuova lega, che pretendesi si stia maneggiando tra le principali potenze marittime del nord, approfittando delle favorevoli disposizioni dell'imperator delle Russie, che impiega ogni studio per coltivare la buona corrispondenza ed amicizia col sultano.

Nella presente oscurità delle cose, nell'incertezza degli avvenimenti ed in circostanze cotanto critiche per noi, ho creduto di dover sottoporre alla vostra saggiezza anche le semplici voci che si spargono in questa lontana regione, acciocché osservando voi più da vicino il corso politico degli affari, possiate dare alle medesime quel peso che meritar può la maggior o minor loro probabilità e trarne qualche utile lume.

Vi unisco una memoria pervenutami dal fu console di Smirne Luca Francesco Cortazzi. Il dovere mi chiama ad assoggettarvela, onde possiate dare ai suoi reclami ed alle sue petizioni quel valore che possono meritare.

Troverete pure annessa al presente piego la continuazione delle carte promessevi sulle questioni che vertono tra il capitano Bronza e il console Franchini, riservandomi di trasmettervi le di lui risposte, tostoché mi verranno prodotte.

Della trieste mia situazione non vi faccio replica, giacché abbastanza vi trattenni col precedente numero. Bensì vi accompagno in copia gli atti, segnati dal mio precessore e da me, convalidati dal ri- 
putato parere da questo ambasciatore della Repubblica francese, sopra i reclami dei cittadini capitani Girolamo e Giovanni Milinovich contro il cittadino segretario Camillo Giacomazzi.

Mercoledì scorso si pose in viaggio per la via di terra il suddetto mio precessore, cittadino Ferigo Foscari, onde restituirsi alla patria. Riuscì sensibilissimo al mio animo il di lui distacco, avendo perduto un collega consumato nella difficile carriera diplomatica, adorno di talenti e cognizioni, il quale con patriottico zelo mi fu sempre di utile consiglio nei spinosi affari di questa legazione.

Salute, rispetto e fratellanza.

Pera di Costantinopoli, li 15 fruttifero anno primo della libertà italiana (primo settembre 1797 v.s.)

\section{[38a]}

\section{Libertà Eguaglianza}

Cittadini deputati.

Ho ricevuto le vostre lettere 4 e 22 luglio scorso, alle quali è necessario che la vostra indulgenza non attenda in questo momento che un semplice riscontro.

Ho già partecipato alla Municipalità, come potrete vedere dal mio dispaccio, quanto ho creduto opportuno di farle sapere, e soltanto a voi aggiungo particolarmente che dalla carta pervenuta da Petrsburg e delle notizie che mi accompagnate, farò quell'uso che prudenza mi consiglierà, assicurandovi in pari tempo che l'amor della patria è fervido nel mio seno e che farò quello che il di lei servigio esige, e che mi viene dal dovere prescritto.

Salute, rispetto e fratellanza.

Pera di Costantinopoli, li 23 fruttifero anno primo della libertà italiana (9 agosto [recte: settembre] 1797 v.s.) 


\section{9}

(Senza inserte)

\section{Libertà Eguaglianza}

Cittadini municipalisti.

Le vostre lettere lungamente attese che in data 21 giugno decorso dovevano recarmi le necessarie istruzioni, mi giunsero soltanto il 7 corrente, dopo che col corriere di Vienna mi erano già pervenute le posteriori dei 22 luglio.

Non so negarvi, cittadini municipalisti, che non sia stata di molta compiacenza al mio spirito lo scorgere dalle stesse che voi, confidando nel mio attaccamento, mi incaricavate di far quei passi che aveva già io stesso segnati. Accesi e voi ed io da un medesimo zelo, ci siamo, benché in tanta distanza, congiunti in viste uniformi; erano state prevenute le vostre istruzioni; m'ero unito d'interesse con questo ministro francese; avea assicurato questo impero della costante amicizia dei veneti e, alla nuova dell'invasione dell'Istria e della Dalmazia, avea tentato di scuoterlo dal letargo ed animarlo all'aspetto del proprio pericolo, come già vi ho rassegnato. Non ostante, al vedermi nelle dette lettere ripetuta la necessità di spingere i maneggi e di accorrere ai bisogni della patria travagliata ed afflitta, conobbi che qualche altra cosa da me si esigesse, e sollecito mi portai alla casa di Francia.

Ivi, colla base del trattato concluso tra la nostra e la Repubblica francese che mi avete spedito, cercai prima di levar il dubbio, che si era introdotto nello stesso ambasciatore, che l'indicata occupazione potess'essere di concerto col general Buonaparte, e che forse si designasse dal Direttorio di trattenersi l'isole del Levante, e poscia lo scongiurai di unirsi a me per far verso la Porta quelle rimostranze efficaci che l'urgenza richiede, onde non rimanga oziosa spettatrice dello spoglio dei suoi fedeli vicini. Impegnato, com'è veramente, questo illustre soggetto per i nostri vantaggi, vide con giubilo che un trattato di pace assicura dal canto della sua nazione la nostra esistenza. Ma, per altro con dolore, mi significò ch'esso non avea ricevuta alcuna istruzione sul nostro rapporto, che, se poteva contar sopra di lui in quanto riguarda gli affari correnti, non dovea in qualità di ministro esporsi a positive rappresentanze compromettendo se stesso, e che gli era necessario di esser a ciò autorizzato da commissioni precise. Sebbene abbia trovato ragionevoli siffatte risposte, rimasi però afflitto dal profondo dolore poiché calcolo quanto debbo la sua cooperazione, né so quale esser possa l'utile effetto dei miei maneggi isolati. Quali essi sieno per essere non saprei dirvelo io stesso in questo momento; il tempo ristretto non mi dà luogo a riflettere e a pena tanto spazio mi resta a potervi rispondere. Le carte capaci a render accorto questo ministero dei danni che gli sovrasta 
le ho sparse tutte nella mani di quelli che ho riputati i più propri al risentimento e ad influire nelle determinazioni. Le disseminerò per altro accortamente con maggior profusione; mi servirò dei nuovi documenti che mi pervennero; vi aggiungerò le più calde insinuazioni e farò in oltre quel più che potrò, come ne sarete avvertiti. Non è già che abbiate a dubitare che la sensazione prodotta dall'invasion austriaca non sia stata riflessibile: vi ho già accennato il cangiamento del reis-effendi; che si sono tenuti dei consigli di stato; che si è spedito un corriere estraordinario a Vienna, e di più vi notifico che in questo gabinetto continua l'azione prodotta dall'avvenimento. Ma vi sono all'incontro i maneggi dei ministri dei coalizzati, e dall'ultimo mio numero avrete rilevato quali sieno le voci qui sparse e come si tenti di distrar i riflessi della Porta dall'esame dei suoi pericoli reali allarmandola sui supposti disegni francesi. Giova sperare ch'essa conosca i suoi veri nemici; ma chi sofferse indolente il partaggio della Polonia difficilmente si determinerà in questo momento a soluzioni d'energia e di vigore.

Non vi parlo dello stato di questa missione per non rinnovarvi il quadro dolente che vi ho fatto negli altri miei numeri. Debbo soltanto dirvi a mia indennità che nella circostanza attuale non è affatto possibile d'attenersi per intero ai metodi vecchi. Vi ho già indicato che molti dei capitani che ritornano dal Mar Nero si ritengono le spedizioni straniere, giacché non reputano di loro vantaggio il riprender le venete. Alcuni però degli stessi richiesero dei passaporti personali, dicendo che non trovandosi sui bastimenti che in qualità di passeggeri, era ad essi necessario l'aver un documento comprovante la loro nazionalità. Doveano e poteano esser negati, ma si deveniva ad un contatto delicato e spinoso, poiché protestavano di dirigersi alla cancelleria austriaca e di dichiararsi imperiali. Per allontanar tale scandalo giudicai partito di prudenza di non rifiutarmi, e spero approverete le mie direzioni.

Già ho un saggio luminoso della vostra bontà e condiscendenza in mio riguardo nella lettera suddetta 22 luglio decorso. La vostra approvazione ai miei passi è la più dolce delle ricompense e non rimane al mio cuore, per esser pienamente contento, che il veder secondati da un esito felice gli sforzi che per il ben della patria sono impiegati da voi e da quelli che tentano di emularvi.

Anche questo ambasciator francese fu estremamente lusingato dalla pienezza delle vostre espressioni che gli ho comunicate, e quindi ho presentemente il conforto di potervi ricambiare colle proteste della sua riconoscenza e del suo attaccamento.

Salute, rispetto e fratellanza.

Pera di Costantinopoli, li 23 fruttifero 9 agosto [recte: settembre] 1797 v.s . anno primo della libertà italiana 


\section{[39a] Senza numero}

(e senza inserte)

\section{Libertà Eguaglianza}

Cittadini municipalisti.

A tenor di ciò che vi ho scritto nel mio dispaccio di numero 34, si dirige a codesta parte il cittadino Ferrigo Cameratta, ministro economico della missione, a cui affido la presente perché ve la rimetta di persona.

Egli sostenne l'uffizio qui affidatogli con quell'onore, abilità e zelo che manifestò sempre negli altri alla di lui fede costì appoggiatigli in passato. Quindi essendone io testimonio veridico, debbo proclamarne il merito e chiamar sull'egregia sua condotta, sul suo patriotismo i vostri riflessi.

Non vi parlo dell'oggetto della sua venuta, giacché abbastanza l'ho espresso nel numero antedetto, ma soltanto vi paleso il mio conforto di veder in lui conoscitor profondo dell'argomento, e che sa bilanciare le circostanze mie con quelle in cui versa l'erario. L'organo più fedele dei miei sentimenti.

Munito com'egli è di tutti i documenti più veridici e sull'economico della legazione, e sul particolare della mia amministrazione, vi farà appieno conoscere quali sono gl'indispensabili pesi per sostenere questa rappresentanza.

Io confido che darete ascolto a quanto sarà per esporvi, come se fossi io stesso, e che lo accrediterete presso quei comitati che fossero competenti, affinché soddisfar possa l'oggetto della sua comparsa.

Sinché sia diversamente disposto, egli quantunque lontano vi renderà conto regolarmente di quest'azienda, avendo preso seco lui le opportune misure.

Non dubitate, cittadini municipalisti, la sua rettitudine, i suoi lumi vi somministreranno la base più certa alle vostre determinazioni, ed io le attendo rassegnato perché saranno figlie dell'equità e quali le esige della patria il servigio.

Salute, rispetto e fratellanza.

Pera di Costantinopoli, li 23 termale, 13 settembre 1797 s.v., anno primo della libertà italiana 
[39b]

(Con una inserta)

Libertà Eguaglianza

Cittadini deputati.

Dopo di avere significato col mio numero 27 la necessità in cui mi trovavo in conseguenza del funesto avvenimento di Smirne di far ripatriare un numero di nazionali dei quali questo governo solennemente mi protestò di non voler tolerare l'ulterior stazione in questa capitale, e dopo di aver pure collo stesso numero e con il successivo numero 29 indicate le misure da me prese a quest'oggetto interessante, mi sono quindi riservato il metodo da me tenuto in questo propositi, onde non compromettere li riguardi reciproci fra li due governi e provedere ad un tempo alla sicurezza dei nazionali. Partendo già da costì il mio ministro economico, avrà seco li documenti tutti necessari alli dettagli che potessero occorrere, così rapporto al suddetto articolo, come all'altro pur relativo e dipendente dalla causale stessa della missione del proconsole a Smirne, sopra i quali entrambi articoli le incontrate spese sono quiditate nel foglio che vi unisco.

Questo foglio medesimo comprende altre tre partite, quella cioè di un giannizzero corriere spedito a Scutari con li pubblici pieghi, e da di là ad Ancona per Venezia, dell'acquisto fatto ad esempio di questo ministro di Francia e nelle stesse misure di una statuetta rappresentante la libertà, posta in luogo cospicuo di questo palazzo, e la spesa finalmente di un nuovo sigillo per imprimerne l'immagine sulle pubbliche carte.

Nella speranza che vi risultino indispensabili le prime, non confutabili le seconde ne confido la vostra approvazione.

Salute e fratellanza.

Pera di Costantinopoli, li 27 fruttifero anno primo della libertà italiana (13 settembre 1797 v.s.)

\section{[39c]}

\section{Libertà Eguaglianza}

Cittadini deputati.

Nel breve spazio di tempo corso dopo la partenza dell'ultimo corriere di Vienna, non mi fu possibile di aver ancora verun riscontro di alcuni segreti maneggi intavolati per sapere qual senso abbiano prodotto i scritti relativi all'Istria e Dalmazia, che accortamente con maggior profusione ho fatto giungere ai più influenti ministri della Porta, 
onde regolare i miei passi a seconda delle risultanze. Mi limito dunque verso la Municipalità alla replicata del numero 39, cogliendo per altro questa occasione per formare alle vostre ultime lettere una più diffusa risposta. Niente di più soddisfacente per me, cittadini deputati, se sortisse di scuotere dal suo letargo questo gabinetto a vantaggio della giusta nostra causa per un avvenimento che compromette gli stessi suoi interessi. Ma come sperarlo se sono privo dell'importante appoggio dell'ambasciatore di Francia, che non ha alcuna commissione, e in mezzo ai ministri della coalizione, che impiegano più scaltri raggiri per cercar di render dubbia alla Porta la mia politica rappresentazione? Ciò non ostante posso assicurarvi che l'intenso amore per la patria, l'energia che n'inspira la vostra indulgenza, i lumi e le istruzioni avanzatemi, tutto confluisce ad animar i miei sforzi. Debbo frattanto assoggettarvi il riflessibile discorso tenuto dal dragomano della Porta al cittadino dragomano Ralli dietro la lettura delle memorie relative al suaccennato argomento. Nel manifestare che i detti scritti erano piantati sopra solide ragioni, disse che non sapeva concepire timori riguardo alla Porta, che, considerando la poca popolazione e gli angusti limiti delle suddette provincie, comprendeva quanto la casa d'Austria, veniva a perdere per le cessioni che far dovea; che però, ben lungi di divenir più potente, la conosceva sommamente indebolita, e in conseguenza meno temibile; che non sarà facile che divenga una potenza marittima, perché l'interesse dell'altre nazioni vi si opporrà sempre; che la marina ottomana è ora, e sempre più si porrà, sopra un piede imponente e rispettabile; che la buona armonia sussistente fra la Porta, la Russia e la casa d'Austria, convalidata da continue riprove, non poteva esser compromessa per un avvenimento che in ragion politica poco interessar poteva il Divano; che bensì era sensibile vedendo prossima ad esser cancellata dalla classe delle potenze una nazione amica, e con la quale la Porta, a fronte di tanti rapporti finittimi, passava da lungo tempo d'una perfetta intelligenza. Aggiunse poi che l'imperatore non avrebbe occupate le suddette provincie senza che la Francia ne fosse in prevenzione intesa, onde non rendere contingenti i preliminari di pace e che, quantunque i due ministri della Repubblica francese e di Vienna, qui residenti, non avessero ricevute per anco uffiziali notizie, egli sapeva da fonti sicuri esser così la cosa. A siffatti discorsi, che penetrarono l'animo del cittadino Ralli, facendo uso della sua esperienza, de' suoi lumi e delle istruzioni ricevute, non lasciò di contrapporre i più forti ragionamenti e di spargere delle dubbietà sopra i fatti dal dragomano della Porta asseriti con tanta precisione. Rifletté altresì che ad esso sembrava impolitica la direzione della Porta nell'astenersi di frapporre la sua influenza per impedir possibilmente che i suoi stati fossero circondati da un vicino potente, che manifestò in ogni tempo le sue male disposizioni, e strettamente legato con un altro gabinetto, le cui mire d'ingrandimento e i progetti sopra l'impero ottomano erano abbastanza 
palesi. Soggiunse infine che sarebbe pericoloso per la Porta il confidare nelle presenti apparenze di tranquillità e il credere che il nuovo imperatore della Russia abbia rinunziato ai piani di chi lo precedette. Debbo nondimeno prevenirvi che l'anzidetto dragomano della Porta mostrossi sempre poco favorevole ai veneti, e che nell'ingratissimo affare di Smirne era uno dei più impegnati per far attribuir loro la principal colpa della commovente catastrofe. Forse anco i discorsi dei stranieri ministri, che ingigantendo i pericoli dipingono i popoli liberi coi più neri colori, hanno fatto concepire al ministro turco non lieve timore nella vicinanza di chi ha la fortuna di vivere sotto un governo costituito sulla base dell'eguaglianza e della libertà. Potrebbe darsi pertanto che il sultano, che avrebbe voluto approfittare dell'occasione per appropriarsi qualche parte dello stato veneto, preferisca ora, che prevede di non potervi agevolmente riuscire, che le provincie confinanti con i suoi domini sieno soggette ad un monarca assoluto, e che quindi partano i discorsi del dragomano della Porta, che sembra tranquillo sull'invasion degli austriaci. Questa è una congettura, che vi assoggetto, perché sia da voi bilanciata nella vostra saggezza, giacché non mi sembra difficile che le accorte istigazioni de' coalizzati possano aver fatta una viva impressione sull'animo di chi si crede nato col diritto di disporre a suo capriccio di molti millioni d'uomini, e che non saprà persuadersi che le libere nazioni per li stessi loro principi rispettano i sistemi dei loro vicini.

Come vi dissi nel passato ordinario di Vienna, ricevetti soltanto nel giorno 7 corrente la vostra dei 4 luglio passato con li pieghi di Francia, perché andarono prima a Corfù e di là passarono in questa capitale. Con essa lettera mi commettete di astenermi dai dispendi. Conosco pur troppo la vostra critica situazione in fatto d'economia, atteso l'esaurimento dell'erario e l'esorbitanza di tanti pesi che n'emergono. Mi compiaccio per altro di aver prevenuti i vostri cenni con le direzioni da me tenute, avendo verificate tutte le restrizioni che poteva combinare colla giustizia, con il decoro della rappresentanza e colle mie facoltà. Circoscritte le spese dell'ambasciata alle unicamente indispensabili, ho sospesa del tutto ogni graziosa pensione, che in passato alcuni individui godevano; quasi per intero l'elemosine, che conobbi per la maggior parte abusate; le mensuali contribuzioni ai dragomani a titolo di minute spese pel maneggio degli affari, che però non cessano di ripetermi e che sono indispensabili, avendo riconosciuto esser forzati ad incontrarle; l'assegno e la tavola del dragomano Mascellini, come maestro di lingua turca; e quantunque nelle due principali cariche di questo governo, come quelle del reis-effendi e di bostangì bassì, siensi cangiati gl'individui, ciò non ostante mi sono astenuto di praticar loro quei doni che in passato si facevano. Ora ch'è di già partito il ministro economico per gli oggetti che ho indicati alla Municipalità col numero 34, sebbene per altro rimanga addetto alla legazione, perché egli solo renderà conto 
anche in progresso della mia amministrazione, dietro le misure con esso prese mi trovo ridotto coi due segretari e un numero di persone necessarie al decoro della rappresentanza. Non pertanto rimangono tuttavia molti aggravi che non si possono scansare. Quattro giovani di lingua, ai quali sono obbligato di somministrar alloggio e tavola. Otto giannizzeri al servigio del palazzo, che riscuotono salari e che ogni volta che s'impiegano, lo che è frequentissimo, esigono estraordinarie mercedi. Affitto di casa di campagna, necessaria per sottrarsi dal mal contaggioso. Affitto della cavana per la custodia dei caicchi; paga mensuale al caicchiere e a dieci remiganti turchi tutte le volte che occorrono; tre capigì, ossia guarda portoni ai due palazzi e alla porta che conduce alla strada di Pera; un guardafuoco; sei portantini, tutti con salari e panatiche. Vi è inoltre la spesa riflessibile del carteggio, approfittando, come faccio, di tutte le poste per ispedirvi i miei pieghi, e di quello che, come capo della nazione, debbo tenere incessantemente con li consoli e loro agenti di tutte le scale del Levante ottomano. Così pure la spedizione frequente di appositi messi, particolarmente nei sei mesi di villeggiatura, per chiamar al veneto palazzo dragomani, giovani di lingua, capitani, loro equipaggi ed altri individui della nazione per la trattazione dei loro affari. Tutto questo cadendo sopra la classe di spese ordinarie; nascono poi frequenti eventualità ch'esigono straordinari dispendi.

Il cittadino ministro economico ha seco tutti li lumi occorrenti a riconoscere il passato e a regolare il venturo; ma intanto l'urgenza è ridotta agli ultimi termini, e tuttavia mi mancano li riscontri della Municipalità sulli suffragi che mi rendono indispensabili a poter sussistere.

Salute, rispetto e fratellanza.

Pera di Costantinopoli, 30 fruttifero anno primo della libertà italiana -16 settembre 1797 s.v.

\section{0}

(Con una inserta)

\section{Libertà Eguaglianza}

Cittadini municipalisti.

Dopo ricevuto il vostro dispaccio 21 giugno decorso, il quale, come vi accennai nel numero 39, così tardi mi giunse, tutte le mie direzioni furono rivolte ad incontrare gl'importanti oggetti ch'esso contiene.

Quindi, giacché avea posti in opera de' secreti maneggi, giacché avea fatte accortemente giungere delle insinuazioni ai più influenti nel ministero e diffuse insieme con avvertenza le carte, di cui mi 
forniste, meco stesso disegnava di procurarmi una conferenza col reis-effendi onde, accreditando maggiormente colla mia presenza le parole dei dragomani, tentar anche per tal via di fargli comprendere a quali passi era chiamato questo impero per assicurare la propria esistenza. Se però un tale colloquio non poteva esser appoggiato dall'intervento e dagli offizi dell'ambasciator di Francia, per non essersi da lui ricevute le istruzioni che voi mi faceste sperare dovergli essere rilasciate, vidi almeno necessario che da lui fossero approvati i miei passi, e perché le vostre commissioni portano ad unirmi a far causa comune con lui, e perché una sola parola di sua disapprovazione avrebbe resi inutili i miei tentativi. // Vedendo per altro che ai discorsi replicatamente, e con insistenza, avanzati l'ambasciatore suddetto con parole evasive si sotraeva dal manifestarmi il proprio pensiero, mi posi in una ragionevole apprensione e fui agitato da dubbi affannosi sui vantati concorsi, sicché reso incerto sulla condotta che avessi a tenere, attendendo impaziente qualche nuova istruzione, e infatti mi pervenne ultimamente col corriere di Spagna li 22 del mese decorso. Allora pienamente compresi il mottivo della condotta dell'ambasciatore e, vedendomi obbligato ad impiegare ben altro che pubblici e solenni modi per servire la patria e sostenere i di lei interessi, di un arcano profondo coprii le mie mosse, onde nella critica situazione in cui ci troviamo schivo possibilmente li contrasti. ॥

Perciò ai veramente esperti ed abili dragomani Giovanni Ralli e Francesco Eugenio Franchini, sopra la fedeltà dei quali pienamente riposo, significai le nuove dirette e pressanti commissioni che avea ricevute ed, infiammando nei bisogni della patria il loro fervore, incaricai il Ralli di procurarsi una segreta conferenza col reis-effendi, ed il Franchini d'impiegare i più forti offizi verso la ragguardevole persona del capitan pascià, tanto attaccato al sultano. Eglino per conto loro corrisposero alla mia aspettazione e non lasciarono intentato alcun passo; ma non corrisponde ai miei voti l'effetto delle loro fatiche e de' loro travagli. Vi esporrò quanto poterono coll'uso della maggior accortezza ritrarre, onde si possa quindi dedurre da quale spirito sia mosso questo governo, quali molle ci vorrebbe per ridurlo all'azione e quanto si debba nello stato presente di cose giudicar il medesimo.

Ottenne il Ralli di poter inoservato parlar liberamente al reis-effendi sudetto. Non vi ripeto, perché immaginar lo potete, il discorso che gli tenne, il qual fu accompagnato da destrità di modi e di blande insinuazioni, ma cauto ed avveduto il ministro ottomano, quale infatti lo rappresenta la fama universale, mostrò bensì molta sensibilità per il danno dei veneti, ma non altro che parole vaghe gli sortirono di bocca, indicando che non erano ancora decisi gli affari e che non si devea perdere la speranza. Rimarcò per altro il Ralli che, sortendo dalla stessa conferenza, si ordinava dal reis-effendi che gli fosse chiamato il dragomanno della Porta, a cui si accorse che gli avrebbe comunicati i discorsi tenuti e ch'egli perciò credette di attendere per 
accertarsene ed avere qualche lume. Non s'ingannò infatti nel pensiero poiché, sortito il dragomanno della Porta dalla stanza del reiseffendi, accennò al Ralli ch'era inteso di tutto e che desiderava parlargli. Ritiratisi pertanto ambidue esso dragomanno, con un tuono non solito, poiché non si mostrò mai molto amico de' veneti, attestò al Ralli il vero senso di dolore che avea la Porta per l'occupazione fatta dagli austriaci e per l'incertezza dello stato delle isole del Levante, ma lasciò per altro travvedere che rimaneva fermo nell'opinione già manifestata, che tutto fosse stabilito di concerto dalle due potenze. // Sulla base allora del trattato da noi concluso con la Repubblica francese, e facendo insieme conoscere che il di lei interesse si opponeva all'ingrandimento dell'imperatore, procurò in ogni modo il Ralli di far vedere la impossibilità del concorso, al che in una maniera ferma rispose il dragomano che il bailo facesse dunque evidentemente costare che i francesi non aveano acconsentito alla cessione, che allora si avrebbe veduto cosa avesse saputo fare la Porta. ॥

Passò in seguito a chiedere se da me si sapea qual era il destino di Parga, Prevesa, Vonizza e Butrintò, su di che essendogli stato risposto ch'io non ne avea alcuna notizia, gli fu dal Ralli soggiunto che però non dava fede alla sparsa diffamazione che gli ottomani disegnassero d'impadronirsene. Allora protestò il dragomano che i possessi dei veneti non sarebbero mai stati occupati dalla Porta, la quale desiderava che restassero agli stessi ma, stroncando però con prontezza il discorso sopra tal proposito, ritornò a parlar della Dalmazia e dell'Istria, indicando che questo governo avrebbe potuto riceverle in deposito finché si fosse deciso l'affare. Forse che questo è il pretesto che medita la Porta al caso che disegni di occupare i luoghi suddetti, poiché dirà che li tiene in deposito per restituirli ai veneti quando le altre potenze facciano lo stesso delle provincie occupate. Questa è una mia congettura, ma che trovo pur troppo ragionevole, come suppongo altresì che tutto il discorso del dragomano suddetto sorta dalla bocca del reis-effendi, anche per le forme con cui fu pronunciato.

Se questo è quello che all'abilità del Ralli venne fatto di operare, non si mostrò meno impegnato il dragomano Franchini nel secondare le mie intenzioni. Avvicinossi al capitan pascià e con insinuanti maniere gli espose lo stato dei veneti e gli fece tutti quei riflessi che poteano destare in lui l'effetto propostosi, valendosi della conoscenza che ha del carattere ambizioso del comandante. Tutto fu ascoltato dallo stesso colla maggior attenzione, ma gli rispose che nulla avea detto che non fosse stato esaminato e discusso e che non si conoscesse con precisione dalla Porta, soggiungendogli poscia se avea da me ricevuti altri ordini, se dovea null'altro comunicargli e che si spiegasse pure senz'alcuna riserva.

Voi ben vedete, cittadini municipalisti, che questo era lo scoglio che dovevamo prevedere, e che avea infatti previsto; ma quali commissioni potea dare al Ralli, potea dare al Franchini, onde il primo 
rispondesse alle obbiezioni del dragomanno della Porta e l'altro potesse tentare un maneggio col comandante suddetto? Niuna, poiché niuna ce ne permette l'attual infelice nostro stato. Quindi ambidue dovettero in silenzio restarsi, niuna cosa potendo con sicurezza affermare, niuna cosa con fondamento proporre. Permettete però che a vostro lume esponga anche quello che la destrità del Franchini ritrasse dal dragomanno del suddetto capitan pascià, e che insieme vi aggiunga le notizie che con non minore impegno si procurò d'altre fonti. Così vi risulterà più chiaro il quadro che vi sottometto, e le conseguenze potranno dedursi da maggior dati. // Fu lungo il ragionamento del dragomano, ma vi rifletterò soltanto che egli diede non poco peso al vedere che nelle date delle carte sortite dalle municipalità del Levante si chiami la loro libertà, libertà ionica, come potrete vedere dall'annesso passaporto, dal che argomento che, se non siano trattenute dai francesi, esista il disegno di formare una repubblica separata, e che dichiarò che tutte le potenze aveano risentito un vivo dispiacere per l'occupazione dell'Istria, Dalmazia ed Albania e per l'ingresso de francesi nell'isole suddette, ma particolarmente il governo inglese. Le notizie poi che il Franchini seppe procurarmi per altre vie, sono che il capitan passà non poté contenersi dal mostrarsi fortemente irritato quando nei fogli di Francofort vide la corrispondenza del general Buonaparte col Mainoti, forse artifiziosamente mentita dal gazzettiere, sicché, facendo interrompere la lettura che gliene faceva il di lui dragomanno, lo spedì immediatamente, non si sa però con quali ordini, al gran visir ed al reis effendi. ॥

Parimenti fu allo stesso indicato che siensi rilasciate delle commissioni al comandante della flottiglia esistente sulle coste della Soria perché debba senza ritardo alcuno condursi in questo porto, dove si allestiscono due fregate e quattro corvette, per spedirle nella acque della Morea. Si dice che dal sangiacato di Salonicco si facciano marciare delle truppe nella penisola suddetta e che vi si destina pascià Genazè Hassan pascià, fu visir al campo nell'ultima guerra coi russi, incaricandosi il musselim, ossia vice-pascià attuale, di passar a Lepanto, e venendo insieme destinati due pascià e due cadì, l'uno in Napoli di Romania, l'altro a Patrasso. Infine si vuole che alla nuova qui giunta, che gli austriaci abbiano occupato anche le Bocche di Cattaro ed il restante dell'Albania veneta, si sia dalla Porta ordinato all'attuale pascià di Scutari Hibraim, fratello dell'ucciso Machmud, di non insister più oltre contro i montenegrini per vendicar sopra d'essi il sangue del proprio fratello, venendo anzi incaricato di pacificarsi coi medesimi, riaprir loro le communicazioni e permettere che, come in avanti, possano approvigionarsi nelle terre ottomane di quanto abbisognano.

Di tutte le notizie suddette, e molto di più dai discorsi tenuti dagl'indicati ministri, sembra a me che dedurre si possono le sei conseguenze seguenti: 
Prima, che questo governo abbia una verace dispiacenza del partaggio di cui sembra minacciato lo stato veneto.

Seconda, ch'esso agirebbe forse, se non credesse che le operazioni de' francesi e degli austriaci fossero concertate, e che non temesse quindi di doversi compromettere non con una, ma con ambedue le potenze, e forse anco colla Russia, attesi gli stretti legami che passano tra la stessa e la casa d'Austria.

Terza, che presentemente l'artifizio de' coalizzati gli fa più temere d'aver vicini ai suoi stabilimenti dei popoli liberi democratici, particolarmente se fossero uniti ai francesi, che l'ingrandimento dell'imperatore per l'occupazione dell'Istria, Dalmazia ed Albania.

Quarta, che non sembra disposto di far alcun passo decisivo, quando non fosse assicurato dalla cooperazione o de' francesi uniti agl'italiani, o almeno degl'italiani soli.

Quinta, che il piano ora stabilito, analogo anche alla situazione e debolezza dell'impero, è di stare sulla difesa, da una parte blandendo i montenegrini, perché non si gettino in braccio agli austriaci, e dall'altra inviando delle forze nella Morea e nell'Arcipelago, onde impedire che i greci sudditi ottomani, non abbraccino i principi di libertà e non si uniscano ai loro fratelli.

Sesta, che nutra forse il disegno, col pretesto da me suindicato, d'impadronirsi dei luoghi di Parga, Prevesa, Vonizza, Butrintò, Cerigo e Cerigotto, dei due ultimi dei quali, anche per detto dello stesso ambasciator francese, non si sa che il general Gentili si prenda alcuna cura, ma sembrano abbandonati a loro stessi.

Può darsi che m'inganni nel calcolo, e desidero anzi ardentemente di potermi ingannare, ma pure, nello stato attuale delle cose, questo è quello che si presenta al mio spirito.

Ho procurato di avvicinare il ministro di Prussia per rilevare quali sieno i suoi pensieri, onde regolarmi a tenore di quanto scoprissi. Ma, se con gentili parole mostra della dispiacenza per la nostra situazione, non lascia però travveder (o perché manchi d'istruzioni, o perché voglia celarsi) qual sia l'impegno che il suo sovrano possa prendere nell'avvenimento. Non mi è però possibile di vedere quello d'Inghilterra. Egli ha con me troncato qualunque corrispondenza, e si espresse liberamente che non potea veder di buon occhio i veneti, giacché le loro navi si uniscono a quelle dei francesi per accrescerne la forza contro la sua nazione. Egli è intimamente unito coll'internunzio cesareo, qualunque sia il senso, a tenor delle parole del dragomano del capitan passà, prodotto nel gabinetto inglese dall'invasion praticata dagli austriaci, e coll'internunzio medesimo passa parimenti d'una perfetta intelligenza anche il ministro russo. Esso internunzio affetta di far credere che tutto sia seguito col concerto dei francesi, e non si può dubitare che in ciò non sia secondato da quest'ultimo. Ambidue essi ministri si contengono meco con apparente gentilezza, ma mi è noto che si sono espressi che mi riguardano soltanto come persona 
privata. Infatti hanno fatto sapere che non riceverebbero alcuna nota ministeriale, ma, non ostante, per non interrompere il corso degli affari, le cancellerie corrispondono fra esse come facevano innanzi.

Si sa in aggiunta che l'internunzio accarezza gli schiavoni, e promette assistenza e favore, riservandosi di chiamarli formalmente alla sua cancelleria quando gli sieno pervenute le istruzioni che i suoi dipendenti spargono dover giungere fra poco.

Questo, o cittadini municipalisti, è il lagrimevole quadro che debbo presentemente offrirvi da queste contrade. Desidero col maggior ardore che possano in qualche modo cangiarsi le circostanze, e che mi si apra una via di essere più fruttuoso al servigio della causa comune. Intanto non cesserò di far ogni sforzo e d'impiegare tutte quelle persone che possono essere vantaggiose ai nostri oggetti. Tra quelle non addette al ministero delle quali ultimamente mi valgo, si distingue il cittadino dottor Giovanni Andrea Zuliani, il quale col favor della sua professione anche sotto l'altro governo era noto non solo per i suoi estesi rapporti verso i principali ministri di questa corte, ma insieme per il suo sincero attaccamento agl'interessi dei veneti. Ora poi che, seguita essendo la nostra rivoluzione, ha il conforto di vivere sotto quella forma di governo richiesta dai suoi principi e dalla sua educazione, spiegò tutto quel fervore, quell'energia, quel patriottismo, che desiderar si potevano. Destramente avvicina le persone potenti in questo paese, diffonde i lumi che nei libricciuoli che mi avete spediti sono raccolti, e cerca, per quanto puote, di destar negli ottomani quell'energia che converrebbe in questi momenti. Egli merita per tutto ciò ch'io lo presenti ai vostri riflessi, e ben volentieri e per un'intima persuasione soddisfo a quest'atto di pura giustizia.

Salute, rispetto e fratellanza.

Pera di Costantinopoli, li dieci vendemmiatore anno primo della libertà italiana (primo ottobre 1797 v.s.)

\section{[40a] Senza numero}

(Con due inserte)

\section{Libertà Eguaglianza}

Cittadini municipalisti.

Debbo assoggettar alle vostre considerazioni una petizione prodottami dal cittadino Francesco Antonio Corner, ch'esercita a questa parte la professione di medico. Egli sotto l'antico governo procacciossi il merito di travagliare per sette anni continui al servigio della squadra destinata in Mediterraneo, come ve lo comprovano legali documenti che rilasciati gli furono, e che infatti vi accompagno in copia. 
Se la sua salute permesso gli avesse di continuar quella carriera fino al momento che la squadra suddetta fu richiamata,, le sue fatiche non sarebbero state senza compenso, ma chiamato da altri pensieri alla cura importante della propria conservazione, sparse inutilmente il travaglio e venne a procacciarsi il sostentamento in queste contrade.

Ora però nella libertà della patria, infiammato esso pure d'entusiasmo e di zelo, anela di riunirsi ai suoi confratelli ed impiegar l'opera sua negli oggetti relativi alla sua professione o nell'armata terrestre o negli spedali militari.

Meritevoli d'elogio e d'accoglimento le offerte che vengono dallo stesso prodotte, io ve le accompagno con compiacenza, non potendo nel medesimo tempo negargli per giustizia il voto della mia persuasione sulla sua onesta condotta.

Salute, rispetto e fratellanza.

Pera di Costantinopoli, 10 vendemmiatore anno primo della libertà italiana (primo ottobre 1797 v.s.)

\section{[40b]}

\section{Libertà Eguaglianza}

Cittadini deputati.

Ho ricevuti col corriere di Spagna i quattro ultimi dispacci che mi avete trasmessi, segnati l'uno li 27 luglio in nome della Municipalità, due li 29 del mese stesso e l'altro li 5 agosto prossimo scorso. A quello scrittomi in nome della Municipalità, ed agli articoli relativi all'interessante argomento contenuti nell'ultimo dei 5 agosto suddetto, vedrete qual è la risposta che da me si forma nel separato numero 40. In questo poi credo di dovervi significare prima che ho pervenuti i desideri del cittadino Carlo Camerata nell'inviare a codesta parte, ma per gli oggetti per altra, e colle riserve antecedentemente indicate, il di lui fratello Ferrigo, e che mi sarà a cuore il sostener possibilmente le vostre disposizioni rapporto al negoziante armeno Giovanni Serpos, e poi di dovervi trattenere sulla parte economica, giacché da voi mi si commette di restringere possibilmente il ministero e di adattarmi, per quanto può comportarlo la decenza di questa rappresentanza, all'attual povertà democratica.

Avea meco stesso stabilito di non parlarvi più direttamente sul punto economico, giacché il cittadino Cameratta suddetto potrà con maggior precisione fornirvi dei lumi più sicuri per le successive determinazioni, ma mi veggo costretto ad allontanarmi da questo disegno quando voglia saper di preciso i vostri desideri per incontrarli con tutta esattezza. 
Avea appena la Municipalità assunto la cura del provvisionale governo, che tutte allo spirito mio si affacciarono le angustie a cui facevasi incontro, e compresi tra l'altro che l'esaurimento dell'erario dovea travagliarla non poco, attesa anche la necessità di sottostare a dei pesanti estraordinari dispendi.

Quindi riconobbi decorso il concorrer al suo sollievo per quanto potea e col troncar le inutili spese e coll'usar il maggior risparmio nelle necessarie, e coll'allontanar tutte le persone che dipendessero da me e che non erano assolutamente indispensabili al servigio della patria.

Io sono di costà partito con due segretari, un ragionato e suo coadiutore, un cavallerizzo, che fungea anche le veci di maggior di provincia, un medico, un chirurgo, un teologo ed un cappellano, giacché ometterò di parlarvi di altre persone che, parte per bisogno e parte per sistema e per ricchezza, poteano seguire questa allora pomposa rappresentanza.

Qui poscia rinvenni un'infinità d'individui, ma non vi dettaglierò il ministero soltanto. Già nella mia lettera dei 16 decorso vi ho fatto cenno di quelli che sono con altro carattere addetti a questa missione, ve li connoterà più particolarmente il cittadino Cameratta esso stesso, sicché è inutile affatto che presentemente vi trattenga su di essi.

Vi dirò dunque che trovai cinque dragomani qui stabiliti, cioè i cittadini Giovanni Ralli, destinato a trattare i politici affari della Porta; Francesco Eugenio Franchini, deputato alla dogana mercantile e ai maneggi col capitan passà, tribunali e magistrati ottomani; Giovanni Maria Mascellini, trattenuto come maestro di lingua e dei giovani che sono a questa parte a tal effetto inviati, e che si presta talvolta alle ispezioni dei due suddetti quando o da incomodi di salute, o d'altre cagioni, sieno impediti, essendo in pari tempo utile quando occorra sul momento d'interpretar le rimostranze che vengono fatte dai turchi; e poscia Carlo Medun e Pietro Naveri, ai quali non fu appoggiata finora veruna particola ispezione.

Non vi parlo d'un altro dragomano, ch'è il cittadino Giuseppe Franchini, poiché richiamato da Smirne, ove fungeva l'officio di console, non è di alcun peso come dragomano alla pubblica cassa, finché non venga deciso da voi del di lui destino, poiché le leggi dispongono che sieno sospese le paghe ai dragomani ch'esercitano qualche consolato.

Otto poi sono i giovani di lingua a questa parte esistenti, cioè i cittadini Giovanni Battista Navon, interinalmente pro-console nella scala di Smirne suddetta, il quale, allorché si trovava in questa capitale si occupava utilmente da molto tempo della traduzione della carte turche; Giovanni Antonio Franchini, che assiste nella sua faticosa missione il fratello maggiore; Domenico Choch, Lorenzo Sanfermo, Marcello Agapito, Paolo Mascellini e Marin Calavrò, che studiano la lingua turca sotto la direzione del dragomano Mascellini ed il giovane Carlo Franchini, che già molto avanzato nella lingua turca, araba e persiana non attende che l'opportunità di porsi in un attivo servigio. 
Sopra gl'individui che meco condussi, e pei quali potea esser libero l'esercizio della mia facoltà, io ho fatte le restrizioni maggiori. L'abile offiziale Francesco Dorsan, capitano d'artiglieria, che fungeva l'officio di maggior di provincia e di cavallerizzo, come ho detto di sopra, l'ho con apposita lettera inviato a disposizione del Comitato militare, e confido che siasi giunto a quest'ora.

Ho già lasciati in libertà il cittadino dottor Pietro Bondioli, il chirurgo Giuseppe Vivardi, il teologo abbate Saverio Scrofani ed il cappellano don Giuseppe Cervini, sicché essendo poi per i motivi suggeriti partito per costà il cittadino Ferigo Cameratta col suo coadiutore, restano soltanto i due cittadini segretari Francesco Alberti e Sebastiano Rizzi, ambidue i quali sono assolutamente necessari per gli affari della legazione e per il bisogno de' nazionali nella troppo molesta attività di questa cancelleria, giacché posso supplire al bisogno d'un cappellano col chiamar quando occorre un sacerdote di questo paese.

Debbo a tutti i suddetti un giusto tributo di lode per la rassegnazione che usarono nell'adattarsi con prontezza alla proposta partenza, poiché al primo cenno riconobbero necessario di non aggravar nelle circostanze attuali, e colle panatiche e cogli onorari che aveano la pubblica economia. A lode del vero si compete per altro in primo luogo l'elogio del cittadino Pietro Bondioli, di cui credo solo che basti indicar il nome per presentar un modello del vero patriota.

Le regolazioni suddette per altro non poterono da me venir eseguite tutte in una volta, poiché combinar dovetti i riguardi pubblici alle particolari convenienze di cadauno dei suddetti, come altresì nello spogliarmi di tutti i domestici non necessari era doveroso seguendo appunto le providenze sul proposito da voi stabilite che attendessi o che si fossero d'altro servizio proposti o che avessero l'opportunità dell'imbarco per tradursi alla patria, coi soccorsi che vennero ad essi da me somministrati.

Se però mi condussi in tal modo verso gl'individui, non mi credetti, e non credo infatti di esser autorizzato a far alcuna innovazione essenziale rapporto ai dragomani e giovani di lingua senza aver prima da voi le commissioni relative.

Ha fatto per altro qualche riguardo ad essi qualche cosa per diminuir i dispendi, come vi ho antecedentemente accennato, perché mi vi obbligò la ristrettezza delle mie circostanze.

Tutti i suddetti ritraggono da costà i loro onorari, ed a peso del bailo non cadevano che le cibarie dei quattro giovani di lingua, che sono isolati, e dalle loro famiglie lontane, la tavola per il maestro di lingua e lo stipendio per tal carico assegnato, e le spese mensuali che i dragomanni Ralli e Franchini sono obbligati di fare nel prestarsi ad eseguire le commissioni della carica.

Ho pertanto ai due dragomanni suddetti soppresso di dar qualunque somma, e gli aggravi cadano presentemente a particolar loro peso; al maestro di lingua Mascellini ho parimenti trattenuto l'onorario e le ci- 
barie ma quand'egli sia obbligato di supplire alle veci di alcuno dei due suddetti debbo somministrargli di volta in volta la somma che occorre perché non si trova in grado di sostener la benché menoma spesa.

Quanto poi ai giovani di lingua suindicati, per un pentimento d'umanità, di giustizia ed anco per decoro della nazione, non potei far alcun cangiamento per non vederli in braccio alla disperazione e perché non fossero astretti di mendicar altrove il loro alimento.

Se però, cittadini deputati, mi venga meno il vostro soccorso, non so a quale deliberazione sarò ridotto dall'urgenza delle circostanze e quale esser possa lo stato della mia e dell'altre persone, che corrono meco un eguale destino.

Conosco pur troppo che somma esser deve la vostra ristrettezza, ma siansi per mezzo il dirvi, affinché possiate saper il tutto che dalle due cambiali, delle quali col ragguaglio che m'indicate avete ordinato il pagamento, io non ne ritraggo soccorso veruno.

Esse furono da me inoltrate sotto l'antico governo, quando la situazione d'allora e la pompa di quest'ambasciata esigevano un dispendio riflessibile; di tutti i dinari delle cambiali stesse secondo l'antico ragguaglio, e di somme anzi maggiori, io sono debitore al mio procuratore cittadino Mangilli, per le spese incontrate nel mio allestimento e per gli avanzi fattimi, e quindi io mi trovo nell'imbarazzo di prima.

All'arrivo forse del presente sarà giunto a codesta parte il cittadino Cameratta, ed egli vi proverà con documenti senza eccezione questa pur troppo insopportabile verità.

Sarà pertanto dalla vostra equità il far quello che troverete conveniente in ciò che ha riguardato al decoro, e per l'avvenire dopo aver fatte le restrizioni che vi ho già significate, farò anche quelle ulteriori che voi giudicherete a proposito, onde rendervi possibilmente meno gravose questa missione.

Salute, rispetto e fratellanza.

Pera di Costantinopoli, 10 vendemmiatore anno primo della libertà italiana (primo ottobre 1797 v.s.)

\section{1}

(Con una inserta)

\section{Libertà Eguaglianza}

Cittadini municipalisti.

In continuazione al mio numero 40 formo nella parte politica col presente risposta ai dispacci che mi furono trasmessi li 26 agosto, 2 e 9 settembre decorsi. Mi riuscirono grate e utilissime le interessanti notizie che mi vennero avanzate, e lessi avidamente le note prodot- 
te al Direttorio a Parigi dal nostro ministro, cittadino Sanfermo, da quello della Repubblica cisalpina e dall'ambasciatore ottomano, poiché con moltissima compiacenza vidi sottoposte al governo francese quelle ragionate considerazioni che il mio patriotismo desiderava.

Da quella dell'ambasciator ottomano vedrete comprovato appunto quello che mi credetti in dovere di assoggettarvi col mio numero anteriore suddetto. Vi si leggono espressi il dispiacere della Porta per l'invasione avvenuta; il suo dubbio che vi sia concorso il general francese; il suo timore, se ciò si avverasse, di compromettersi in faccia alle due potenze; il suo desiderio perciò che la Francia si spieghi, e d'essere assistita dalle forze della stessa per determinarsi allora a quei passi che vede necessariamente richiesti dal proprio interesse.

Alla lettura d'essa carta, che mi svelava quello che con tanta oculatezza non solo da me, ma insieme a questo ambasciatore di Francia si tenne nascosto, credetti opportuno consiglio di presentarmi con una nota alla Porta coll'apparente motivo di attestarle la riconoscenza della nazion veneta per i passi fatti in di lei vantaggio dall'ambasciatore esistente a Parigi.

Come però rileverete dalla stessa, ben diversi furono i veri oggetti per cui la produssi. Mi restava a cuore di rinovar più da presso, ed anche con un mio scritto, il quadro dell'importanza delle provincie occupate; voleva diminuir la sensazione di timore che i coalizzati hanno cercato di destare in questo gabinetto dalla vicinanza di popoli democratici; bramava di sapere quali erano le misure prese verso la casa d'Austria e di eccitare inoltre il governo a passi di vigore e di forza verso il Direttorio francese.

Ve l'accompagno in copia perché possiate giudicarne, confidando nella vostra indulgenza; ed eccovi insieme le risposte che l'abile dragomano Ralli ritrasse dal reis-effendi e dal dragomano della Porta, a cui anche privatamente feci tenere le carte che mi avete trasmesse.

Ambedue i suddetti riprotestarono che la Porta conserva per i veneziani una vera amicizia; fu dato il conveniente risalto alla costante fedeltà mostrata dalli stessi nell'osservar i trattati e alla cura con cui coltivarono la buona intelligenza; non si mostrò di dubitare che tale non dovesse essere la condotta avvenire, ma quando s'insistette dal Ralli per saper qualche cosa circa le misure prese e che si prenderanno contro la casa d'Austria, allora venero in campo le riserve, le fiacche voci e il dire che si facea dalla Porta quanto da lei dipendeva, ripetendoci però molte volte dentro i limiti del proprio potere.

// Il dragomano per altro, stretto dal Ralli più da presso, gli aggiunse qualche cosa di più, quello cioè che voi ed io prevediamo, poiché gli disse che se le due potenze belligeranti si sono accordate sulla di lei rovina, sarà assolutamente impossibile l'impedirla, ad onta di tutti li tentativi che possa fare questo governo. ॥

Esso non è in caso di agir con vigore e di porsi a qualche cimento con più d'una potenza, e il nostro destino dipende dalle negoziazio- 
ni di Passerian, che voi forse a quest'ora saprete. Già anche d'altre relazioni avute dopo la partenza dell'ultimo corriere, e prima della produzione della memoria, mi era confermato che tali erano i sentimenti di questo gabinetto. Esso teme, ve lo ripeto, d'esporsi non solo contro l'imperatore e contro i francesi, ma forse anco verso la Russia, e giorni fa il reis effendi richiese una conferenza al ministro di quest'ultima potenza, e si dice che l'oggetto possa essere stato il procurar d'indagare in qual modo pensi la corte di Pietroburgo sulla occupazione fatta dalla casa d'Austria e se si possa assicurarsi che non sia per prender una parte attiva in favore della stessa, al caso che questo impero fosse obbligato a ricorrer all'uso delle armi.

Lo stesso capitan pascià in una domanda che fece al cittadino Franchini, spiegò evidentemente il dubbio che ha il suo sovrano di compromettersi. Io lo coltivo con ogni studio impiegando l'attività del dragomano suddetto e confidenzialmente gli feci tenere copia della memoria che produssi e delle carte che voi mi spediste.

Gli fu spiegato appena il contenuto delle stesse, che richiese con ansietà, se i ministri veneto e cisalpino aveano ottenuta risposta, indicando che il loro ambasciatore l'attendeva ancora, e si rilevò che al comandante importava molto l'assicurarsi intanto delle disposizioni de' francesi. L'incertezza in cui è questo governo su questo rapporto trasparisce anche dalla condotta che tiene verso questo degno ambasciator generale Aubert Dubayet. Esso generale in pubblica assemblea con amarezza si dolse che la Porta con vani pretesti si rifiuti dar la libertà ai tre cittadini francesi fatti prigionieri sopra un corsaro di Malta, mentre, in aggiunta alle tante prove d'amicizia date alla sua repubblica a questo governo, vi sieno quelle della protezione accordata in tutta l'estensione dell'Italia ai sudditi ottomani, che preservò ad essa tanti millioni; la liberazione dei prigionieri africani ch'esistevano al Zante; lo stesso di quelli ch'erano a Corfù; e il libero passaggio a spese della nazione procurato a tanti ottomani che, fuggendo dagli austriaci, si erano ricoverati nel territorio della repubblica. Forse che le risposte che avranno ottenute a quest'ora i tre ministri a Parigi, e le ultime determinazioni del direttorio al general Buonaparte, tranquilleranno interamente la Porta, togliendo anco ogni equivoca interpretazione alla corrispondenza del general sudetto coi mainotti e ad alcune espressioni dei suoi proclami in Italia, che i malevoli, per quanto ritrassi, rappresentarono come offensive i riguardi di questo impero; ed allora uniti dal comune interesse vedremo rassodati i vincoli di perfetta amicizia e di stretta alleanza.

Nel precedente numero 40 vi ho indicato che qui non si sapeva qual fosse il destino dell'isole di Cerigo e Cerigotto, le quali si credeano abbandonate a esse stesse. Ora per altro posso darvi qualche riscontro sulle deposizioni di alcuni di quegli abitanti di colà ultimamente partiti. Al semplice invito del cittadino francese Guyz, ex-conso- 
le della sua nazione ed ora municipalista del Zante, non vollero quei di Cerigo aderire alla riforma e rispedirono le lettere avute. Ai 22 di agosto però, secondo alcuni, o ai 26 dello stesso, secondo qualche altro, giunse all'isola stessa una leggera fregata veneta con paviglione francese, da cui sbarcarono il comandante e due altre persone. I suddetti si portarono dal provveditore, vi lessero delle carte e fu conseguenza della lettura che vi eressero l'albero della libertà, su cui spiegossi la bandiera francese, essendosi però dagli abitanti assunta la coccarda italiana. Fu in seguito istituita una municipalità composta di sedici persone; si congedò il veneto ex-patrizio; vi si ordinarono le cose, e quando si vide assicurata la tranquillità e contento il popolo, partì il comandante francese senza lasciarvi altro presidio che la soldatesca che vi era innanzi, incaricando però come delle funzioni di agente un vice-console della sua nazione, che è nativo del luogo, il quale si chiama Giorgio Leguni. L'isola di Cerigotto non era democratizzata ancora alla partenza delle persone assunte, ma non aveano alcun dubbio che non lo fosse quanto prima, e fecero vedere dai loro passaporti che vi si data dall'anno primo della libertà citerea.

Vi do questo dettaglio poiché forse non vi sarà così facile l'averlo altronde, e vi aggiungo di più che ho eseguite le vostre commissioni e verso l'ambasciatore di Francia e verso i nostri confratelli dell'Istria, Dalmazia e Albania.

Il primo è sensibile alle vostre espressioni, ma tra i secondi poi soltanto alcuni ai miei vivi eccitamenti ripresero coraggio, ed attendono avidamente delle più accertate notizie e più consolanti; ma degli altri, di tempra non ferma, se non ricusano affatto di riconoscermi, fanno però troppo fiocamente risuonar sulle labbra i dolci nomi di libertà e d'uguaglianza, e si mostrano vinti dalle arti dei vostri avversari.

Salute e rispetto e fratellanza.

Pera di Costantinopoli, li 25 vendemmiatore anno primo della libertà italiana - 16 ottobre 1797 v.s.

\section{[41a]}

\section{Cittadini deputati.}

Sebbene sia certo che la vostra saggezza non lascierà di avanzarmi le opportune istruzioni, perché a tenore dei risultati delle negoziazioni di Passeriano o di pace o di guerra, o che dobbiamo essere uniti o star disgiunti dalla Repubblica cisalpina, io possa avere una norma sicura nella mia condotta avvenire, mi credo in dover non ostante di cooperare dal canto mio alla maturità delle vostre determinazioni col sottoporvi alcuni pochi riflessi che mi dettano le conoscenze locali e l'esperienza in questo offizio acquisita. 
Venni commesso da voi di rimaner al mio posto sostenendo le prerogative, il titolo e il rango annessi al bailo di Venezia, benché dopo la seguita rivoluzione non mi abbiate fatte tenere le nuove credenziali da prodursi al sultano. E di fatto, o che la Porta, sensibile alla disoluzione della veneta Repubblica, sia poi stata indifferente al cangiamento avvenuto nel governo, o che abbia cercato di guadagnar tempo per attender prima di decidersi che si diradi la presente politica oscurità, fu forse vantaggiosa la nostra riserva perché vincemmo gl'intrighi de' coalizzati e si continuò a riconoscermi per veneto rappresentante; gli affari e alla Porta e ai tribunali ottomani vennero trattati col sistema di prima, e si dissimulò la notoria separazione dell'ex-provincie venete della terraferma, l'invasione dell'Istria, Dalmazia ed Albania, e le misteriose direzioni dei francesi e delle rispettive municipalità dell'isole del Levante dopo la loro rigenerazione.

Potrebbe darsi che a questo effetto abbia insieme concorso l'antica denominazione di bailo di Venezia, che così appunto mi firmo nelle pubbliche carte, poiché questo titolo non potea presentare in ordine ministeriale alcun ostacolo alla Porta per non aver a continuare la sua corrispondenza colla repubblica, qualunque fosse l'estensione de' suoi stati.

Se però continuarono e continuano col vecchio piede le cose, ora che non è noto per anco lo sviluppo dei politici affari, non è per altro possibile che al momento, che sia conosciuto, progredir si possa sullo stesso sistema, e quindi mi sono per allora necessarie delle commissioni chiare, precise ed alle circostanze adatte.

Se a seconda del voto generale de' popoli, e come infatti ricerca l'interesse comune, abbia luogo la nostra unione colla Repubblica cisalpina, ridotta allora Venezia a un semplice dipartimento, non può più avere una particolare rappresentanza alle corti, quantunque sia per essere il centro della repubblica.

Dietro questa ipotesi, se non venissero evacuate l'Istria, la Dalmazia e l'Albania, e se l'isole del Levante non riputassero di unirsi cogl'italiani, questi non avendo più gl'interessi che noi avevamo inanzi colla Porta, non crederanno forse necessario di far risiedere a questa parte un ministro.

Allora, in qual maniera dovrò io contenermi verso la Porta? Come verso i consoli coi quali sono in corrispondenza e che sono subordinati a questa missione; come verso i nazionali e verso le figure che mi sono addette?

Merita parimenti un serio riflesso la cancelleria, ossia segreteria, che qui esiste. Vi si contengono dei documenti di varie sorte, cioè dispacci, decreti, lettere missive e responsive, varie carte turche, carte ed atti di affari definiti; carte ed atti di affari pendenti, depositi di dinaro e effetti, la maggior parte appartenenti o agli schiavoni od ai greci. Nella medesima situazione, se si eccettui la corrispondenza politica col governo, si trovano parimenti le cancellerie dei consoli suindicati. 
Inoltre, come dovrò diportarmi verso i dragomani e i giovani di lingua? Quali disposizioni dovrò fare onde custodire il pubblico palazzo ed impedire il suo deterioramento nello stato di disordine in cui si trova per la debolezza della sua costruzione? Cosa stabilire perché non rovinino affatto le di lui già pericolanti adiacenze?

La mia condotta infine verso i ministri, e particolarmente verso quello di Vienna, quale dovrà essere? Voi ben comprendete, cittadini deputati, che questi sono tutti articoli essenzialissimi, e che sopra gli stessi e sopra gli altri ancora, che nella vostra prudenza conoscerete opportuni, mi si renderebbe in quel caso d'aver una norma sicura.

Se poi la Repubblica cisalpina (abbia o no unite le suddette provincie) creda di suo decoro che vi sia alcuno che a questa parte la rappresenti, dovrà allora la persona incaricata di tal onore sostenere il rango, le prerogative, i privileggi che ha presentemente il bailo e che competerebbero alla nuova repubblica, attesa la sua associazione colla veneta.

Questo è un argomento del più serio riflesso ed è necessario che per base delle comunicazioni, alle quali sarete allora determinati, che io va faccia noto in primo luogo di qual carattere sieno rivestiti i membri componenti questo corpo diplomatico.

Oltre il bailo, ossia ambasciator di Venezia, vi sono presentemente altri due ambasciatori soltanto: quello di Francia, che a tutti precede, e quello di Olanda, sul quale ha il bailo la preminenza.

Da rimotissimi tempi risiede a questa parte un ambasciatore francese, e soltanto vi fu qualche cangiamento nel corso della rivoluzione, attesa l'emigrazione del Choiseul, che tradendo la patria abbandonò vilmente il suo posto. Allora fu spedito il cittadino Descorches, ma questi non fu riconosciuto come ministro che poco prima della sua partenza, e trattò per molto tempo gli affari, presentando memorie e petizioni e assistendo i suoi connazionali, senza che fossero accolte le sue lettere di credenza. Il cittadino Verminac, che gli succedette, spiegò il carattere d'inviato estraordinario finché poi, destinato a questa parte il general Aubere Du-Bayet, fu a lui, secondo l'uso del regime antico, conferito il carattere spezioso d'ambasciatore.

Quello d'Olanda è lo stesso, che qui si trova dal tempo dello Statolder. Benché non abbia presentate ancora lettere di credenza, la Porta lo riconosce come ambasciatore, non essendo forse per essa un obbietto la variata costituzione, mentre l'Olanda rimane repubblica come prima, ed è probabile che si attenda il momento d'aver una solida costituzione per fornirlo di credenziali; e frattanto quello di Francia, come ministro di una repubblica alleata, lo assiste in tutti gli affari.

La casa d'Austria poi tiene qui un internunzio; la Russia un inviato e ministro plenipotenziario; lo stesso la Prussia e Napoli; la Svezia un inviato; la Spagna e la Danimarca un incaricato d'affari, come lo ha pure da qualche tempo l'Inghilterra, sebbene abbia eletto un ambasciatore. 
Quando pertanto la Repubblica cisalpina si sarà determinata, in confronto delle potenze suddette, sul carattere che vorrà conferire al suo ministro, le converrà poscia stabilire, qualora lo volesse di primo rango, se questi debba sostenere le prerogative godute dal bailo di Venezia, e singolarmente quello dell'ingresso solenne, proprio a lui solo, e per la quale, come rileverete dai miei primi scritti all'antico governo, ho avuti degli ostacoli alla mia istallazione. Parimenti converrà che si determini se nelle visite al visir e al sultano per la presentazione delle credenziali, e al momento dell'ingresso suddetto, se mai abbia luogo, debbano continuarsi i doni di prima, e se siano da impiegarsi i soliti mezzi verso il ministro ottomano nell'occasione di dover trattare affari importanti.

Un esame più maturo ancora meritano le capitolazioni. Esse sono in molte parti non solo ambigue ed oscure, ma dannose agl'interessi della nazione, e converrebbe in ogni maniera tentare di migliorarle.

Avvertite, cittadini deputati, che io non vi faccio che dei piccoli cenni relativi alle conoscenze, che non possono ch'essere particolari di chi è nel maneggio delle cose, né intendo di presentarvi un quadro di ciò che sul rapporto di questa missione dovrete al caso comunicare, poiché l'estensione dei vostri lumi non ha bisogno del mio debole soccorso e, se vi ho parlato appositamente della capitolazioni, mi v'indussero gli ostacoli ch'esse mi presentano ogni giorno nell'assistere i miei connazionali.

In continuazione per tanto di quello che vi ho esposto, al caso si verificasse la conclusione della pace, permettete che poche cose vi aggiunga insieme, nel supposto che dovessero essere riprese le ostilità.

E allora assolutamente necessario che risieda a questa parte un ministro, onde servir possa prima al grande oggetto d'indur la Porta a quei passi di vigore e di forza che il bisogno richiede, e poscia per tenerla ferma nelle prese misure e concertar possibilmente l'unione e nell'esecuzion e nei mezzi.

Questo per altro deve esser condotto da commissioni precise, e le sue direzioni non possono senza svantaggio essere lasciate all'azzardo, come lo rileverà evidentemente la vostra penetrazione.

Potrei sopra i suddetti articoli dirvi molte altre cose, ma mi limito, come vi ho indicato di sopra, a farvi soltanto i cenni, che sono assolutamente necessari, nella riserva di aggiungere alle prime ricerche tutto quello che voi credete a proposito di rilevare più fondatamente.

Salute, rispetto e fratellanza.

Pera di Costantinopoli, li 25 vendemmiatore anno primo della libertà italiana (16 ottobre 1797 v.s.) 


\section{VIII computati i due segnati coi numeri 32 e 33}

\section{Libertà Eguaglianza}

\section{Cittadini Deputati.}

Il negoziante Serpos, di cui mi parla il vostro dispaccio 29 luglio decorso, avea già temuto fino dal principio del cangiamento di governo, che lo si avrebbe assoggettato ad una contribuzione, e prevedendo il momento avea a questa parte avanzati degli offizi pressanti per ischermirsi.

Avrete ciò rilevato da quanto vi scrissi col mio numero 37, in cui comunicandovi la risposta che si diede alla nota da me prodotta per assicurare un amichevole accoglimento del convoglio destinato per Alessandria, vi significai insieme essersi dal dragomano della Porta soggiunto che confidava che i veneti vi corrisponderebbero coll'aver i convenienti riguardi per i soggetti ottomani esistenti in codesta città, indicando tra essi nominatamente il Serpos.

Dalla confidenza che gl'inspira un tal appoggio deriva pertanto la sua recredenza di pagar la somma a cui venne tassato, e come una tal circostanza merita un serio riflesso, permettete che mentre attendo che si parli sull'argomento per eseguir con tutto l'impegno gli ordini vostri, vi sottoponga intanto alcune considerazioni atte a farvi conoscere fino a qual punto senza pericolo d'inconvenienti potrò spingere i maneggi per sostenere le vostre determinazioni.

Sarebbe incontrattabile [nella replicata: incontrastabile] quanto voi m'indicate sulla base dell'articolo XIII del trattato di Passarovitz, se l'articolo stesso avesse avuto la sua litterale esecuzione. Essendovi espresso che gli andanti e venienti negli stati ottomani non abbiano a pagar il carazzo, a cui per altro saranno soggetti quelli che si stabilissero, dovrebbe per la reciproca, ancorché non espressa, esser tenuto alla contribuzione il Serpos, perché da molti anni costì stabilito.

Il fatto però è molto diverso in vantaggio dei veneti dal contenuto nella capitolazione. Non furono soltanto esenti dal carazzo gli andanti e venienti, ma un tale vantaggio lo godettero, e lo godono sin ora una quantità d'individui qui stabiliti e ammogliati, anzi varie e varie famiglie che contano molto lontana l'origine veneta, e per sino dei sudditi ottomani, che l'antico governo prevalendosi dell'inavvertenza di questa corte, arbitrariamente credette di rinovar all'ombra della sua protezione. Venivano alli stessi rilasciate delle ducali, e vengono detti perciò ducalisti; ed in tutte le scale del dominio ottomano ve ne hanno non pochi.

Dopo l'affare di Smirne, allorché un incendio maggiore ardeva contro la nostra nazione, temetti sui rapporti particolarmente del console nella scala suddetta, che si volesse esaminar con serietà l'affare, e scrissi costì per aver istruzioni. Fortunatamente per altro si venne a capo d'acquietare la Porta, e la maggior parte dei medesimi conti- 
nua a godere degl'antichi privilegi, tra i quali è però necessario che a vostro lume distingua quelli di esenzione dalle tasse personali e gl'altri ch'esentano dal pagare le imposizioni sulle merci nelle misure prescritte per i soggetti ottomani.

Quanto alle tasse personali, di qualunque denominazioni sieno, i veneti tutti, e quelli che si fecero e si fanno passare per tali, ne sono interamente liberi a somiglianza degli altri franchi. Furono rispettati anche nei casi dei maggiori bisogni. Nell'ultima guerra colla Russia fu obbligato cadauno a portar i propri argenti in zecca, dove venivano pagati meno del reale valore, astringendosi quelli che non li portavano a pagar la differenza che passava tra il vero valore e quello dal governo fissato, ed i franchi non furono sottoposti alla pesante gravezza.

Un uguale vantaggio godettero pure coll'esser sottratti dall'altro aggravio che in tal incontro fu imposto, di allestir cioè un corpo di caliongì per equipaggia le navi da guerra, il quale cadde soltanto a peso delle nazioni greca ed armena.

Non si possono per altro chiamar esenti equalmente a norma dei trattati di pace che tutte le nuove estraordinarie imposizioni daziali. I veneti, per esempio, dopo aver pagato sopra le merci che acquistano, o vendono, il tre per cento di dogana e la messetteria, non dovrebbero sottostare ad alcun altro peso.

Non vengono già asoggettai dai firmani della Porta, né si può dire che li paghino direttamente, ma lo fanno per altro indirettamente, ch'è la medesima cosa, spezialmente nei cotoni, filati, vini, lane, sete, caffè di Francia, etc. Entrino o sortano le mercanzie sudette debbono passar per la dogana e l'ottomano, o acquirente o venditore, viene alla imposizione obbligato. Quindi, se acquista valuta gli aggravi che deve sostenere, e paga tanto meno la merce; se vende, altera il prezzo comprendendo l'importo dei nuovi dazi, di maniera che in ultima analisi pagano infine anche i franchi.

È vero che l'esazione per conto pubblico non è così sicura per i generi suddetti come lo sarebbe se fosse diretta, perché in tal modo possono avere ed hanno infatti luogo più facilmente i defraudi, ma non ostante i franchi non godono realmente di tutti i vantaggi che sono dalle rispettive capitolazioni ad essi accordati.

Questi miei riflessi sull'aggravio che indirettamente soffrono i franchi, e per conseguenza anche i veneti, potrebbero nel maneggio riguardo al Sorpos essere di qualche utilità, se fosse diversa la natura della contribuzione che imposta gli venne. Ma non per questo, siatene certi, ometterò di fare al momento quanto potrà dipendere da me, avendo però sempre innanzi agli occhi l'oggetto espresso nelle vostre saggie istruzioni di non esporvi ad una rappresaglia.

Salute, rispetto e fraternità.

Pera di Costantinopoli, li 4 annebbiatore anno primo della libertà italiana - 25 ottobre 1797 v.s. 
P.S.

Col corriere di Vienna ho ricevuto il vostro numero 13, che avvisa il recapito di tutti i miei dispacci sino al numero 37, ma io ho la dispiacenza di annunziarvi che mi manca quello che mi avete segnato con numero 12.

Con quello di Spagna poi mi pervenne la vostra replicata del numero 11, ma ho rilevato ch'è stata aperta nel viaggio, poiché al vostro sigillo n'era stato sovrapposto un altro più grande, e vi aveva le parola di libertà ed eguaglianza.

Sarà bene per tanto che anche per quella strada usiate l'avvertenza di coprire le vostre carte con qualche direzione privata, come farò io pure costantemente, onde sottrarle così con più facilità agli sguardi dei maligni o dei troppo curiosi.

Vedrete marcati i miei dispacci d'oggi coi numeri romani, poiché credo di fare tale distinzione tra quelli che scriverò a voi e quelli che inoltrerò alla Municipalità, nei quali sarà continuata la numerazione già cominciata sotto l'antico governo, e vi avverto che sin tanto ch'io non abbia istruzioni diverse, me le addrizzerò tutte le volte che dovrò riscontrarle quello che mi verrà dalla stessa imposto immediatamente, o che mi sarà da voi qualche cosa in di lei nome commesso.

\section{IX}

\section{Libertà Eguaglianza}

Cittadini deputati.

Mi credo chiamato a darvi un particolare riscontro sui cenni che mi faceste coi vostri numeri 10 e 11 in data 2 e 9 settembre decorso relativamente all'affare di Smirne. Come però, oltre ai dispacci scritti all'antico governo, ne ho parlato a voi pure coi numeri 36, 37 e 38, così sarò breve presentemente e richiamerò le vostra attenzione a quei punti soltanto sui quali compariscono necessarie le determinazioni della Municipalità e vostre.

L'argomento suddetto in quanto riguarda il politico abbraccia due parti distinte, l'una verso la Porta, l'altro verso la corte di Russia.

La Porta che volle attribuire ai veneti la causa dell'incendio avvenuto, e che desiderava rivolger a loro peso le dimande che le faceano di risarcimento i ministri francese e d'Olanda, e quelle che avessero potuto avanzarle dietro un tale esempio anche gli altri, con una carta ministeriale comunicata a tutto il corpo diplomatico imputò solennemente la catastrofe agli schiavoni, zantiotti, ceffaloniotti e corfiotti, pretese che fossero scacciati dalle terre ottomane, mentre avea già prima ricercata la demissione del console. 
Ad un attacco così violento e risoluto mi opposi con quanto vigore mi fu dalle circostanza d'allora permesso. Sostenni che la colpa di qualche scorretto individuo non poteva attribuirsi all'intera nazione, che un semplice omicidio non giustificava l'orribile eccesso della soldatesca ottomana, la quale dai comandanti avrebbe dovuto essere raffrenata, che l'indistinto allontanamento richiesto era offensivo i trattati e a quanto si pratica verso tutte le nazioni franche, mentre avea innanzi significato che non era in mia facoltà di dimetter affatto il console Franchini, poiché dipendeva dal governo che investito lo avea.

Colla sospensione per altro, e col richiamo a questa parte del Franchini stesso, impegnandomi di purgar il dominio ottomano dalle persone sfaccendate e viziose, eseguendo anche l'impegno; ed impiegando in oltre i più caldi ed efficaci maneggi, mi venne fatto di calmar il furore e sofferse la Porta che molte persone delle nazioni suddette a questa parte restassero.

Nel mentre così travagliava a schermirmi dagl'impeti di questo governo, da me condotto al punto suddetto, non era meno angustiato dalla direzione che tennero prima il ministro e poi la corte di Russia.

Non vi ripeterò tutto quello ch'è avvenuto, poiché ne avete un esatto dettaglio ne' miei dispacci anteriori, dai quali insieme vedrete a qual passo mi chiamò l'impegno che aver dovea che non fosse interrotta la corrispondenza colla corte suddetta, passo che allora questo ministro sperava opportuno e sufficiente all'oggetto. Dopo sovravvenne la rivoluzione, e fu sospesa qualunque trattazion d'affari tra noi, sicché non potei col suo mezzo saper l'esito della dichiarazione da me data per rettificare le asserzioni del console. Ma per altro s'io riguardi a quanto da Pietroburgo mi scrisse il cittadino Venier li 15 luglio decorso, posso dedurre dalle parole di quel primo ministro di stato, che l'affar era per aver un termine amichevole e pronto.

Non ebbi in seguito alcun altro riscontro, ma voi per altro ne sarete con più precisione informati, sicché se si avveri quello ch'io suppongo, si può considerare e verso la Russia e verso la Porta terminato l'affare in quanto al politico; quando però si desista dai ministri di Francia e d'Olanda di pretender da quest'ultima il risarcimento dei danni sofferti dai loro nazionali, cosa che non è dalli stesse lasciata di vista.

Giova per altro aprire il nostro cuore alla speranza e confidare che le loro dimande non saranno per reagire sopra di noi, tanto più che gli avvenimenti di costà influiranno per certo anche sopra questo articolo.

Quantunque però si desideri terminato sotto questo aspetto l'affare, voi ben sapete ch'è pensile tuttavia il destino del Franchini in quanto al consolar suo carico, interinalmente sostenuto dal cittadino Navon col carattere di pro-console.

In oltre sopra quanto ho esposto coi miei numeri 36, 37 e 38, dev'essere stabilita la forma di dar corso all'esame delle varie questioni ad esso Franchini promosse. 
Avrete veduto come sono voluminosi i documenti che vi feci tenere, e vedrete che non lo sono meno gli altri che ho raccolti e che vi spedirò quanto prima.

Sieno pertanto anche gli articoli suddetti presenti ai vostri riflessi, tosto che in mezzo alle ocupazioni attuali possiate averne lo spazio.

Salute, rispetto e fratellanza.

Pera di Costantinopoli li 4 annebbiatore anno primo della libertà italiana - 25 ottobre 1797 v.s.

\section{X}

(Con una inserta)

\section{Libertà Eguaglianza}

Cittadini deputati.

Vi parlo in quest'ordinario d'un tuono meno dolente del solito, poiché le nuove qui giunte rinforzano la mia speranza e par che l'aspetto delle cose sia vicino a cangiarsi. Suona nella bocca di tutti la parole di guerra; che lo scioglimento del congresso di Lilla, il manifesto del Direttorio della nazion francese e l'ordine che per il 15 vendemmiatore fossero complete e pronte a battersi le armate, la fanno creder certa ai veri repubblicani e temere agli aristocratici, quando dalla casa d'Austria non si accolgano le condizioni di pace che le vengono offerte. Non potete credere, cittadini deputati, qual sia stato l'effetto prodotto dalle notizie dalle notizie suddette e dal vedersi impressa sui fogli di Francfort la memoria prodotta al Direttorio esecutivo dall'ambasciator ottomano che i ministri di Russia, d'Inghilterra e di Cesare procurarono sul principio di far credere apocrifa. Sembra ch'essi non ne abbiano avuta conoscenza che coll'ultimo corriere di Vienna, giacché dal mio canto ho procurato di non diffonderla, onde ritardar possibilmente gli offizi che avessero potuto tentare per affievolir l'energia della Porta. Ho da sicuri riscontri di persone, che coltivo per rilevare cosa si pensi dai ministri suddetti, che l'internunzio ne fu estremamente agitato e che se ne sorpresero i ministri di Russia ed inglese. Il primo vide, ad onta forse delle significazioni che avrà avanzate alla sua corte, che questo impero conosce i propri interessi, sa distinguere quali sono i suoi veri nemici e che, se la guerra si rinovi, non dormirà tanto inattivo come può darsi che l'abbia fatto sperare; i secondi poi nel linguaggio della Porta ravvisarono uno stile troppo coraggioso e robusto e parve che, svelandosi pubblicamente da un ministro ottomano i disegni che si conoscevano già coltivati, particolarmente dal gabinetto di Pietroburgo, siensi offesi i riguardi che si credono ad essi dovuti. Quindi sì gli uni che 
l'altro si apprestano a chieder alla Porta delle rischiarazioni per rilevarne più fondatamente i disegni e le mire. Riflettendosi da taluno che l'ambasciatore ottomano abbia forse alquanto trascorso nel manifestare in iscritto, che a questo governo sono già noti gli ambiziosi progetti della Russia d'invadere i suoi possessi in Europa e che congiuntamente all'Inghilterra le sarebbe meno difficile il recarli ad effetto, se rimanessero nella mani di Cesare le provincie invase ai veneti, viene persino creduto che ai ministri di Russia e d'Inghilterra sarà per dichiarare aver il ministro a Parigi passati su tal articolo i limiti delle avute commissioni, ma non si giunga però a dubitare che sia per mancar al suo decoro in faccia alla corte di Vienna.

Nutro anch'io la medesima lusinga, spezialmente se si verifichi il cominciamento delle ostilità e se continui nell'ambasciator di Francia l'attività che rimarcai in questi ultimi giorni. Ebbe lo stesso due lunghe conferenze col reis-effendi e, prevalendomi della sua amicizia, procurai di rilevarne il contenuto. Mi disse lo stesso di aver energicamente e con diffusione rappresentati tutti i mali che ne deriverebbero a questo impero se restasse inerte e ozioso al caso che si riaccendesse la guerra; che fu da lui dimostrata la facilità che avrebbero gli ottomani di attaccar Cesare con un esito fortunato, onde a scacciarlo dalle provincie invase e far anche sopra di lui dei considerabili acquisti; che disegnò rapidamente un piano tale d'allontanar i dubbi che le spese nell'esecuzione non fossero tollerabili dalle circostanze attuali dell'erario, giacché fece vedere che una sola armata che marciasse dai confini della Bosnia, dietro le opportune intelligenze col general Bonaparte, sarebbe bastevole all'oggetto; che rimarcò nel reiseffendi una viva sensazione alle fatte proposizioni poiché, fiaccate le forze di Cesare da sì lunga guerra, non poté contraddire che, combattuto ancora dai francesi e dagl'italiani uniti, non offrisse propizia l'occasione a questo sovrano di prender vantaggiosamente l'armi; che lo stesso per altro non si spiegò maggiormente, nella riserva di assoggettar tutto al consiglio di stato ed averne le commissioni, protestando intanto molta gratitudine per l'interesse dimostrato e per i lumi offerti. A ciò mi aggiunse l'ambasciatore ch'ei non diffida d'aver quanto prima una risposta, se non in tutto almeno in parte conforme ai suoi desideri, poiché, sebbene non fosse stabilito di prender al caso attivamente parte nella guerra, non crede che si rifiuterà almeno di spedir una forte armata ai confini, che farebbe anche col solo sospetto di minaccia un'utile diversione. Questa sarà probabilmente la condotta che verrà tenuta dalla Porta, atteso il soverchio suo timore di compromettersi in faccia alla Russia, quando non ottenga dalla stessa delle assicurazioni che la lascino per tal conto tranquilla.

Intanto qui si rimarcano tali disposizioni da creder sicuramente che per la ventura primavera sortirà sul mare una flotta poderosa, e furono anche rilasciati degli ordini per far marciar delle truppe ai confini della Bossina. Mi terrò anche in seguito in osservazione di- 
ligente per rilevare tutto quello di più che possibile sarammi, onde non vi manchino anche da questa parte i riscontri opportuni per servire utilmente alla causa della libertà e della patria.

Vi accompagno una petizione e due attestati prodottimi nel mese decorso dai giovani di lingua stanziati in questa casa nazionale ai quali, dietro il ragguaglio da voi stabilito nell'accettazione delle mie cambiali, credetti di dover comunicare che a soli reali dodici al mese si riduceva quello che veniva da me esatto per l'importar delle loro panatiche. Essi a voce mi esposero e, poscia, come vedrete mi ripeterono in iscritto, che ridotti alla misura suddetta non potrebbero per certo alimentarsi, pregandomi quindi di non far alcuna alterazione al loro riguardo. Conoscendo appoggiata al fatto la loro esposizione, per sentimenti di umanità ho aderito ed aderisco ai loro desideri, finché mi facciate sapere quali saranno sulle domande loro e sul loro destino le determinazioni che prenderete. A questo proposito vi debbo anzi dire che fin sotto il passato governo, conoscendo da un canto l'importanza che dalla buona educazione dei giovani di lingua ne risultino al pubblico servigio degli abili ed esperti dragomani, e dall'altro vedendo che non vi si avea pensato bastantemente, ho fatto degli esami ed ho raccolto delle osservazioni su questo articolo interessante, sicché mi offro di assoggettarle a voi qualunque volta crediate di abbisognarne per istabilire un sistema migliore di quello osservato sin ora.

Salute, rispetto e fratellanza.

Pera di Costantinopoli, anno primo della libertà italiana li 11 annebbiatore (primo novembre 1797 v.s.)

\section{$\mathbf{X I}$}

\section{Libertà Eguaglianza}

Cittadini deputati.

Un corriere estraordinario spedito dall'ambasciator ottomano esistente a Vienna recò a questa parte la notizia che siasi conclusa la pace tra la Repubblica francese e la casa d'Austria. Le condizioni ne sono però ignote, e quindi attendo a mio conforto i vostri riscontri, giacché fatalmente ne fui privo affatto e coll'ultimo corriere di Vienna, ed anche con quello di Spagna.

Vi ripeterò di nuovo che il vostro numero 12 non è giunto alle mie mani, ed il successivo poi dei numeri 13 non fa che accennarmi il ricapito di quelli che v'avea scritti e v'erano fino a quel momento pervenuti.

Credo inutile il rappresentarvi quanto mi sieno perciò necessarie le vostre istruzioni per regolar la mia condotta verso la Porta e ver- 
so i ministri, ed anche nell'articolo interessante dell'amministrazione criminale e civile di quest'ambasciata, a tenore di quanto ho esposto coi miei numeri alla Municipalità 36 e 37.

La cura di prestarsi al servigio della patria negl'importanti politici oggetti è spinosa e difficile, particolarmente nelle circostanze attuali; ma qui in aggiunta sono ogni giorno molestamente distrato dagli studi e dalle osservazioni opportune a tale vista eminente, per combattere contro i raggiri, l'insubordinazione e la licenza, e per garantirmi dalla responsabilità che mi si vuole addossare. Già forse sarò obbligato, finché mi venga l'appoggio delle vostre determinazioni, di prender delle interinali misure onde poner un qualche fermo alla malizia e all'insidie, poiché l'offesa dignità della reppresentanza nazionale, il vantaggio del commercio e la comune tranquillità reclamano altamente un tal passo. Spero di poterne parlar con più precisione nell'ordinario venturo.

Dall'offizio delle segreteria verificossi che per conto dell'estinto marinaio Casson ci è depositata la somma di piastre settecento ottantadue parà venti, e mi sono rivolto a questa dita mercantile Marco ed Abramo quondam Salamon Fuà perché ve le faccia tenere per mezzo dei suoi corrispondenti Lazzaro Jacob Vita e nipoti Vivante. Anche le carte relative al deposito stesso vi verranno assoggettate quanto prima.

Benché dalla Porta non si dubiti che voi non siate per avere tutti i riguardi per i padri e per gli studenti armeni ch'esistono nell'isola di San Lazzaro, e costì nonostante sono ricercato di raccomandarveli efficacemente. Ho fatto rispondere che rende giustizia ai veneti confidando sulla loro amicizia e buona fede, e che comunicherò i desideri che mi furono spiegati, e son certo d'aver con questa risposta incontrate le vostre intenzioni.

Salute, rispetto e fratellanza.

Pera di Costantinopoli, anno primo della libertà italiana li 20 annebbiatore (10 novembre 1797 v.s.)

\section{XII}

(Con otto inserte)

\section{Libertà Eguaglianza}

Cittadini deputati.

Approfitto della partenza del corriere di Spagna per inoltrarvi la replicata nel numero antecedente e per soddisfar l'impegno che mi son preso nello stesso d'accompagnarvi in autentica le carte relative al deposito del defunto marinaio Felice Casson, ch'esistevano nell'of- 
fizio delle segreteria, ed alle quali il cittadino Alberti aggiunse la dichiarazione che vedrete inserta nelle medesime. Collo stesso incontro debbo assoggettarvi una memoria che mi venne prodotta. La cittadina Anna Mascellini, figlia d'un morto benemerito dragomano, è quella che l'avanza. Dall'antico governo, per i servigi prestati dal padre, avea ottenuto un'annua pensione di ducati 120 che le veniva somministrata dalla cassa bailaggia. Le ne venne sospeso il pagamento nelle provisionali regolazioni da me fatte nell'amministrazione, e chiede presentemente che dalla vostra benignità le si continui l'effetto delle grazie ottenute. Fate quelle che riputerete conveniente ed equo, ch'io attendo gl'ordini vostri, come pure fluttuante sui vari discorsi che si fanno rapporto alle condizioni di pace, attendo d'esser da voi positivamente informato e diretto nella mia condotta avvenire.

Salute, rispetto e fratellanza.

Pera di Costantinopoli, anno primo della libertà italiana li 26 annebbiatore (16 novembre 1797 v.s.)

\section{2}

(Con tre inserte)

\section{Libertà Eguaglianza}

Cittadini municipalisti.

Ho ricevuto colla posta di Spagna i dispacci inviatimi li 28 settembre e li 7 ottobre decorsi, e vivamente desidero che la conclusione della pace abbia verificate le vostre speranze e le mie. Qui si tenta di amareggiarmi collo spargere delle nuovi crudeli sulle condizioni stabilite, ma io voglio e debbo attenermi alle sole vostre confortanti parole, poiché la causa della nostra patria è appoggiata alla giustizia ed alla verità e la leale nazione francese non può mancare a se stessa.

Lascio pertanto di farvi per ora altri discorsi sopra questo argomento e passo ad attestarvi i sensi della maggior riconoscenza per ciò che pensate e operate a mio riguardo. Della mia rettitudine e del mio fervore vi posso rispondere, e volesse il cielo che alle intenzioni fossero eguali le forze e le doti. Voi però generosi mi animate colla vostra approvazione, e quindi crederò io stesso di essere non inutile affatto ai comuni interessi, poiché tale è il vostro giudizio. Anche l'impegno che pretende per le mie circostanze economiche nel dimostrarmi la vostra bontà, mi colma d'una tenera confusione. Non ho giammai ambite le ricchezze, poiché forse capaci di far allontanar da un sistema di moderazione e saggezza; ma vorrei possederne in questo momento per non pesare in alcuna maniera sul pubblico erario e per offrirle anzi tutte in doveroso olocausto alla patria. Ma mi 
conviene aver sofferenza, limitare a soli voti il mio patriottismo ed ammirare la vostra benignità.

Farò uso di tutti i lumi che m’avanzate per rassicurar i nostri fratelli e mi prevalerò della confidenza che in me riponete per sostenere col conveniente vigore l'onor nazionale e il decoro di questa rappresentanza. Forse col venturo corriere v'assoggetterò alcune interinali discipline, che ho imaginate, alle quali mi determinarono, oltreché gl'imperiosi motivi che v'accennerò opportunamente, anche le indicibili amarezze che dovetti soffrire per conto dei capitani Nadal Perusino, Elia Vulovich ed Agostino Petrina. Vi saranno accompagnati gli species facti dei loro affari; vedrete l'irregolarità dei loro passi; come si sieno lasciati condurre più dall'intrigo de' propri difensori che dall'equità e dal vero loro interesse, poiché il Vulovich mi obbligò a reclamare l'assistenza dell'ambasciator francese e ho dovuto segnare un decreto d'arresto contro il Petrina. Desidererei di non dovervi parlare giammai di chi ci appartiene, che colle voci di stima fraterna, o almeno di potermi tacere, come faccio di molteplici altri affari, che giornalmente vengono spinti col medesimo spirito, e che cerco di terminare colla maggior moderazione e dolcezza. Ma se vi si oppone l'altrui condotta, se s'insultano con indecenza i ministri della legazione, se si offende nella mia persona chi ha l'onore di rappresentarvi, allora non è più mia, ma nazionale l'ingiuria, il mio silenzio sarebbe un delitto. Debbo dirvi la verità e lo farò mio malgrado.

L'ambasciator francese non cessa di darci continue prove del suo attaccamento. Secondando le indicazioni del cittadino general Gentili, ottenne dal capitan passà una lettera per la Reggenza d'Algeri, onde sieno dalla stessa lasciati in libertà ottantanove veneziani in cambio del numero pari d'algerini che il suddetto generale consegnò al passà d’Iannina. Colla nota segnata numero 1 m’offerse l'ambasciatore o di munir delle convenienti istruzioni l'agente francese in Algeri per conseguirne l'effetto, o di rimettermi la lettera autentica per accompagnarlo a voi. Per i motivi che vi risulteranno dalla nota numero 2, ho significato al detto ambasciatore il mio desiderio d'accettare il secondo partito e, subito che mi sia rimessa, l'inoltrerò a codesta parte, e vi faccio tenere intanto la traduzione al numero 3.

Attendo nuovi motivi onde poter esercitare il mio zelo, e siate certi che non calcolo per giorni di vita che quelli che impiego a servizio dell'adorata mia patria.

Salute e rispetto e fratellanza.

Pera di Costantinopoli, anno secondo della libertà italiana, li 5 brinoso (25 novembre 1797 v.s.) 


\section{Elenco dei dispacci}

ASVe, Senato, Dispacci Costantinopoli, f. 243, nrr. 1-15, 17-24;

Bailo a Costantinopoli, b. 107, nr. 16

1. Dalla fregata Fama, acque di Fisolo, 22 aprile 1796

2. Corfù, 11 giugno 1796

3. Corfù, 13 giugno 1796

4. Corfù, 13 agosto 1796

5. Corfù, 31 agosto 1796

6. Pera di Costantinopoli, 25 novembre 1796

7. Pera di Costantinopoli, 25 novembre 1796

8. Pera di Costantinopoli, 10 dicembre 1796

9. Pera di Costantinopoli, 24 dicembre 1796

10. Pera di Costantinopoli, 24 dicembre 1796

11. Pera di Costantinopoli, 10 gennaio 1797

12. Pera di Costantinopoli, 10 gennaio 1797

13. Pera di Costantinopoli, 25 gennaio 1797

14. Pera di Costantinopoli, 25 gennaio 1797

15. Pera di Costantinopoli, 10 febbraio 1797

16. [Pera di Costantinopoli], 25 febbraio 1797

17. Pera di Costantinopoli, 10 marzo 1797

18. Pera di Costantinopoli, 24 marzo 1797

19. Pera di Costantinopoli, 10 aprile 1797

20. Pera di Costantinopoli, 10 aprile 1797

21. Pera di Costantinopoli, 25 aprile 1797

22. Pera di Costantinopoli, 25 aprile 1797

23. Pera di Costantinopoli, 10 maggio 1797

24. Pera di Costantinopoli, 10 maggio 1797

25. Pera di Costantinopoli, 24 maggio 1797

26. Pera di Costantinopoli, 24 maggio 1797

27. Pera di Costantinopoli, 10 giugno 1797 
ASVe, Municipalità provvisoria, b. 185 (aggiunto il 10 giu. 1958 - ex f. 243)

$\begin{array}{ll}\text { 28. } & \text { Pera di Costantinopoli, } 20 \text { giugno } 1797 \\ \text { 29. } & \text { Pera di Costantinopoli, } 26 \text { giugno } 1797 \\ \text { 30. } & \text { Pera di Costantinopoli, } 5 \text { luglio } 1797 \\ \text { 31. } & \text { Pera di Costantinopoli, } 10 \text { luglio } 1797 \\ \text { 32. } & \text { Pera di Costantinopoli, } 25 \text { luglio } 1797 \\ \text { 33. } & \text { Pera di Costantinopoli, } 1 \text { agosto } 1797 \\ \text { 33a. } & \text { Pera di Costantinopoli, } 10 \text { agosto } 1797 \\ \text { 34. } & \text { Pera di Costantinopoli, } 10 \text { agosto } 1797 \\ \text { 35. } & \text { Pera di Costantinopoli, } 10 \text { agosto } 1797 \\ \text { 36. } & \text { Büyükdere, } 16 \text { agosto } 1797 \\ \text { 37. } & \text { Pera di Costantinopoli, } 25 \text { agosto } 1797 \\ \text { 38. } & \text { Pera di Costantinopoli, } 1 \text { settembre } 1797 \\ \text { 38a. } & \text { Pera di Costantinopoli, } 9 \text { settembre } 1797 \\ \text { 39. } & \text { Pera di Costantinopoli, } 9 \text { settembre } 1797 \\ \text { 39a. } & \text { Pera di Costantinopoli, } 13 \text { settembre } 1797 \\ \text { 39b. } & \text { Pera di Costantinopoli, } 13 \text { settembre } 1797 \\ \text { 39c. } & \text { Pera di Costantinopoli, } 16 \text { settembre } 1797 \\ \text { 40. } & \text { Pera di Costantinopoli, } 1 \text { ottobre } 1797 \\ \text { 40a. } & \text { Pera di Costantinopoli, } 1 \text { ottobre } 1797 \\ \text { 40b. } & \text { Pera di Costantinopoli, } 1 \text { ottobre } 1797 \\ \text { 41. } & \text { Pera di Costantinopoli, } 16 \text { ottobre } 1797 \\ \text { 41a. } & \text { Pera di Costantinopoli, } 16 \text { ottobre } 1797 \\ \text { VIII. } & \text { Pera di Costantinopoli, } 25 \text { ottobre } 1797 \\ \text { IX. } & \text { Pera di Costantinopoli, } 25 \text { ottobre } 1797 \\ \text { X. } & \text { Pera di Costantinopoli, } 1 \text { novembre } 1797 \\ \text { XI. } & \text { Pera di Costantinopoli, } 10 \text { novembre } 1797 \\ \text { XII. } & \text { Pera di Costantinopoli, } 16 \text { novembre } 1797 \\ \text { 42. } & \text { Pera di Costantinopoli, } 25 \text { novembre } 1797\end{array}$

Sono sottoscritti unicamente dal bailo Vendramin i dispacci nrr. 1-6, 8, 10, 12, 14-15, $17-18,20,22,24,26,28-44$.

Sono sottoscritti congiuntamente dai baili Foscari e Vendramin i dispacci nrr. 7, 9, 11, 13, 19, 21, 23, 25, 27 (con l'eccezione del nr. 7 firma prima Foscari e poi Vendramin).

Sono indirizzati al Governo provvisorio i nrr. 28-31 e quindi alla Municipalità provvisoria i nrr. 34-39a, 40, 40a, 41, 42.

Sono indirizzati al Comitato di salute pubblica i nrr. 32, 33, 33a, 38a, 39b-c, 40b, 41a, VIII-XII. 


\section{Nota degli allegati ai dispiacci di Francesco Vendramin}

Nr. 1

Allegati

1. Nota del seguito di Francesco Vendramin (s.d.).

$\begin{array}{ll}\text { Teologo } & \text { L'abbate Scrofani } \\ \text { Cappellano } & \text { L'abbate Cervini } \\ \text { Cavallerizzo } & \text { Il capitanio d'artiglieri Dorsan } \\ \text { Medico } & \text { Il dottor Pietro Bondioli } \\ \text { Chirurgo } & \text { Il dottor Giuseppe Virardi }\end{array}$

Nr. 2

Allegati

1. Nota delle bandiere acquistate a Corfù da Francesco Vendramin per l'impaviglionamento della fregata Bellona destinata per di lui conserva (s.d.).

2. Ricevuta del ragionato Giuseppe Tassini delle merci destinate a Tangeri ed Algeri (1796, giu. 8).

Nota delle bandiere acquistate a Corfù dall'illustrissimo et eccellentissimo signor Francesco Vendramin, bailo alla Porta ottomana, per l'impaviglionamento della pubblica fregata Bellona destinata per di lui conserva fino a Costantinopoli.

Nr. $1 \quad$ Olandese

$1 \quad$ Maltese (e poi sempre 1)

Capricciosa a tria, bianca, gialla e rossa

Detta rigata, rossa, bianca e bleu

Detta tutta blu 
Detta contorno giallo e campeggiata blu, rosso e bianco

Detta rigata rossa e bianco in linee angolari

Detta quadri grandi inquartati bianco, rosso, giallo e bleu e quadri minori inquartati similmente

Spagnola

Maltese con la division del gran maestro

Capricciosa righe bianche, bleu e gialle angolari

Russa imperiale

Capricciosa a scacchi grandi e piccoli bianchi, bleu e gialli

Sardegna

Capricciosa a quadri centrali bianchi, rossi e bleu, con contorno bianco

Detta righe traversali rosse e gialle

Detta due colori bianco e rosso

Detta a fasse tre gialle e rosse con quadri intermedi

Maltese con division del gran maestro differente dalla prima

Olandese

Capricciosa inquartata rossa e gialla

Detta righe bianche e rosse

Detta righe gialle, rosse e bleu

Detta inquartata con quadrelli intermedi bianchi e bleu

Repubblica Francese nella costituzione presente

Inglese

Capricciosa righe bleu, gialle e rosse con contorno bianco

Detta inquartata rossa e gialla

Detta righe bleu e bianche con quadrelli interni

Detta inquartata a triangoli rossi e gialli

Detta rossa e gialla in tre fasse

Detta a quattro fasse con triangoli interni bianchi, bleu, rossi e gialli

Detta righe bleu con piccioli quadretti bianchi

Detta a scacchi bleu e rossi

Detta righe rosse, bianche e gialle con contorno bleu

Detta inquartata bleu e rosso

Detta inquartata bleu e gialla

Detta a triangoli bianchi, bleu e rossi

Detta inquartata bleu e rosse

Detta in tre colori rossi, bianchi e bleu

Detta a colonna bleu e bianca con contorno giallo

Detta a dama bleu e giallo

Detta inquartata bleu, giallo con fassa central bianca e perpendicolar rossa 


\section{Nr. 6}

Allegati

1. Nota degli effetti regalati e spese incontrate da Francesco Vendramin alla Porta ottomana (1796, nov. 25).

2. Perizia di Girolamo Zulian sui danni patiti dalla nave Medea (1796, nov. 8).

3. Lettera di Francesco Vendramin a Zaccaria Bonlini (1796, nov. 11), copia.

4. Nota di effetti regalati a spese incontrate in diverse occasioni da Francesco Vendramin (1796, nov. 25).

\section{Nr. 7}

Allegati

1. Lettera di Angelo Orio, governator di nave (1796, ott. 27).

2. Lettera di Pietro Medun, console in Algeri (1796, ott. 13).

3. Lettera di Giuseppe Franchini, console a Smirne (1796, nov. 12).

4. Lettera di Giuseppe Franchini, console a Smirne (1796, nov. 14).

\section{Nr. 8}

Allegati

1. Traduzione dell'ilam del giudice di Smirne (1796, dic. 1), copia.

2. Traduzione della lettera di Hacı Osman, voivoda di Smirne (1796, dic. 1), copia.

3. Non si trova

${ }^{\star \star \star \star \star \star \star \star \star \star \star}$ trasmesso con lettere del console di Smirne 30 nov. $1796^{\star \star \star \star \star \star \star \star \star}$ data turca primo di gemaziul 1211 (corrisponde al 2-11-1796)

\section{Nr. 10}

Allegati

1. Supplica del sergente Giovanni Damugliano (s.d.)

2. Supplica del sergente maggiore Armeni (s.d.)

3. Supplica del sergente maggiore Grisogano (s.d.)

\section{Nr. 11}

Allegati

1. Nota di vesti e denaro esborsato per il regalo ai bey delle galere giusto il solito (s.d.).

2. Nota ministeriale di Aubert du Baych a Francesco Vendramin (1796, dic. 26), copia.

3. Nota ministeriale di Francesco Vendramin Aubert du Bayet (1796, dic. 27), copia.

4. Terminazione di riconferma di Giuseppe Coch per console a Salonicco (1797, gen. 10).

\section{Nr. 13}

Allegati

1. Nota ministeriale di Aubert du Bayet, ambasciatore francese (1797, gen. 12), copia.

2. Traduzione di biglietto di Costantin Ipsilanzi, dragomanno della Porta (1797, gen. 10), copia.

3. Nota ministeriale di Francesco Vendramin a Aubert du Bayet, ambasciatore francese (1797, gen. 14), copia.

4. Nota ministeriale di Francesco Vendramin al primo dragomanno della Porta (1797, gen. 14), copia. 
Nr. 14

Allegati

1. Nota degli effetti regalati all'ambasciatore tripolino e ufficiali del suo seguito (s.d.)

\section{Nr. 15}

Allegati

1. Lettera di Francesco Vendramin al vice console a Tripoli (s.d.), copia.

2. Nota dei presenti fatti al confidente dell'ambasciatore tripolino (s.d.)

\section{Nr. 18}

Allegati

1. Nota del regalo fatto da Francesco Vendramin al nuovo gran doganiere (1797, mar. 22).

\section{Nr. 19}

Allegati

1. Lettera di Francesco Vendramin $a^{\star \star \star \star}$ (1797, apr. 10)

[dovrebbero essere 12, ma ce n'è uno solo e non sembra nemmeno un allegato Lettera datata 10 aprile 1797 indirizzata genericamente a provveditori e capitani in cui si riassume quanto contenuto nel numero 19]

\section{Nr. 20}

Allegati

1. Traduzione di memoriale di Sadik reis (s.d.), copia.

2. Traduzione d'ilam del giudice di Modone alla Sublime Porta (1796, ottobre), copia. // ultimi della luna di rebiul akhis 1211

3. Traduzione di arz mahzar (supplica) sottoscritta e sigillata dai comandanti civili, militari, dagli uomini di legge e un gran numero degli abitanti di Corone (1796, ottobre), copia.

4. Nota del regalo fatto da Francesco Vendramin a vari personaggi nel bairam grande (1797, mar. 30).

\section{Nr. 21}

Allegati

non si trovano

\section{Nr. 22}

Allegati

1. Nota di spese, mance e dispense di effetti fatte da Francesco Vendramin (1797, apr. 25).

\section{Nr. 23}

Allegati

non si trovano 
Nr. 24

Allegati

1. Lettera di Francesco Vendramin a Carlo Aurelio Widmann provveditore generale da mar (1797, gen. 8), copia.

2. Lettera scritta da Francesco Vendramin a Carlo Aurelio Widmann provveditore generale da mar (1797, apr. 8), copia.

3. Comando imperiale diretto a Giafer, pascià di Valona (1796, luglio), copia.

4. Traduzione di lettera di Giafer, pascià di Valona (1797, mar. 15), copia.

5. Promemoria di Francesco Vendramin alla Porta (1797, mag. 1), copia.

6. Foglio della forza navale ottomana, compresi i legni in attuale servizio e quelli esistenti nelli cantieri (1797, mag. 9).

7. Polizza annuale delle spese ordinarie e straordinarie fatte dal console veneto dei Dardanelli nelle occorrenze del pubblico servizio dal mese di maggio 1795 sino al presente aprile 1796 .

\section{Nr. 25}

Allegati

non si trovano

\section{Nr. 29}

Allegati

1. Nota ministeriale scritta dall'ambasciatore di Venezia alla Sublime Porta (1797, giu. 21), copia.

2. Nota ministeriale scritta dall'ambasciatore di Venezia a tutti i ministri dell'estere corti (1797, giu. 22), copia.

3. Nota ministeriale scritta dall'ambasciatore di Venezia all'ambasciatore di Francia (1797, giu. 23), copia.

4. Nota ministeriale scritta dall'ambasciatore di Francia all'ambasciatore di Venezia (1797, giu. 24), copia.

5. Nota scritta dall'ambasciatore d'Olanda all'ambasciatore di Venezia (1797, giu. 24), copia.

6. Nota scritta dall'ambasciatore di Venezia al ministro plenipotenziario di Svezia (1797, giu. 23), copia.

7. Nota scritta all'ambasciatore di Venezia dall'incaricato di affari di sua maestà re di Danimarca (1797, giu. 23), copia.

8. Nota scritta all'ambasciatore di Venezia dall'inviato straordinario e ministro plenitopenziario di sua maestà il re di Prussia (1797, giu. 23), copia.

9. Nota scritta all'ambasciatore di Venezia dall'inviato straordinario e ministro plenitopenziario di sua maestà il re delle Due Sicilie (1797, giu. 23), copia.

10. Nota scritta all'ambasciatore di Venezia dall'inviato d'affari di sua maestà il re di Spagna (1797, giu. 23), copia.

11. Lettera circolare scritta dall'ambasciatore di Venezia ai consoli, pro-consoli, vice-consoli (1797, giu. 23), copia.

12. Discorso dell'ambasciatore di Venezia ai suoi concittadini (1797, giu. 22), copia.

13. Foglio a stampa del discorso dell'ambasciatore di Venezia ai suoi concittadini (in francese). 
Nr. 30

Allegati

1. Nota ministeriale scritta all'ambasciatore di Venezia dall'internunzio cesareo (1797, giu. 25), copia.

\section{Nr. 32}

Allegati

1. Note ministeriali scritte dall'ambasciatore di Venezia alla Sublime Porta (1797, lug. 17 e lug. 24) e di altra scritta dall'ambasciatore di Francia alla Sublime Porta (1797, lug. 19), copie.

\section{Nr. 34}

Allegati

1. Nota scritta dall'ambasciatore di Venezia alla Sublime Porta (1797, ago. 5), copia.

2. Nota scritta dall'ambasciatore di Venezia alla Sublime Porta (1797, ago. 5), copia.

3. Bollettino a stampa con notizie pervenute alla legazione di Venezia in Costantinopoli.

4. Atto segnato dall'ambasciatore di Venezia (1797, ago. 8), copia.

5. Nota scritta dall'ambasciatore di Venezia all'ambasciatore della Repubblica francese (1797, ago. 8), copia.

6. Nota scritta dall'ambasciatore di Venezia all'ambasciatore della Repubblica francese (1797, ago. 10), copia.

\section{Nr. 35}

Allegati

1. Nota scritta dall'ambasciator della Repubblica francese all'ambasciator di Venezia (1797, ago. 10), copia.

\section{Nr. 36}

Allegati

1. Elenco delle inserte spedite col corriere di Spagna.

\section{Nr. 38}

Allegati

1. Elenco delle inserte già spedite col corriere di Spagna.

Nr. 39a

s.n. Lettera di Federico Camerata dal lazzaretto (1797, ott. 10)

\section{Nr. 39b}

Allegati

1. Nota delle spese fatte dalla cassa bailaggia dal cittadino Francesco Vendramin, bailo alla Porta ottomana, per le sottoindicate ragioni che si rassegnano per la pubblica approvazione compilata da Federico Camerata (1797, set. 9 stil vecchio). 
Nr. 40

Allegati

non si trovano

Nr. 40a

Allegati

1. Supplica del medico Francesco Antonio Corner (s.d.), copia.

2. Varie attestazioni di Leonardo Correr, governator di nave a favore di Francesco Antonio Corner (1789, lug. 1; 1789, dic. 24; 1795, dic. 11), copia.

Nr. 40b

Allegati

1. Attestazione della Municipalità provvisoria di Zante (1797, ago. 15).

\section{Nr. 41}

Allegati

1. Nota ministeriale scritta dal bailo di Venezia alla Sublime Porta (1797, ott. 10), copia.

Nr. X

Allegati

1. Supplica al bailo dei giovani di lingua (1797, ott. 10).

\section{Nr. XII}

Allegati

non si trovano

Nr. 42

Allegati

1. Nota ministeriale scritta dall'ambasciator di Francia al cittadino bailo di Venezia (1797, nov. 19), copia.

2. Nota ministeriale scritta dal cittadino bailo di Venezia all'ambasciatore francese (1797, nov. 22), copia.

3. Traduzione, in francese, di lettera del capitan passà al dey di Algeri (15 luna cemaziullevel 1212 / 15 brumaio anno 6 - 1797, nov. 7).

- Scritture del tribunale correzionale in merito alla causa c/ il console Giuseppe Franchini e copia di decreto della Municipalità (1797, ott. 31), copia. 


Il volume raccoglie i dispacci di Francesco Vendramin, l'ultimo bailo veneto a Costantinopoli. Sono l'occasione per esplorare la comunità veneta nell'Impero in quanto il bailo dovette illustrare ai nuovi governanti della Municipalità Provvisoria, affatto digiuni di politica internazionale, il funzionamento della rappresentanza diplomatica a Costantinopoli e della vita della comunità veneta nell'Impero Ottomano.
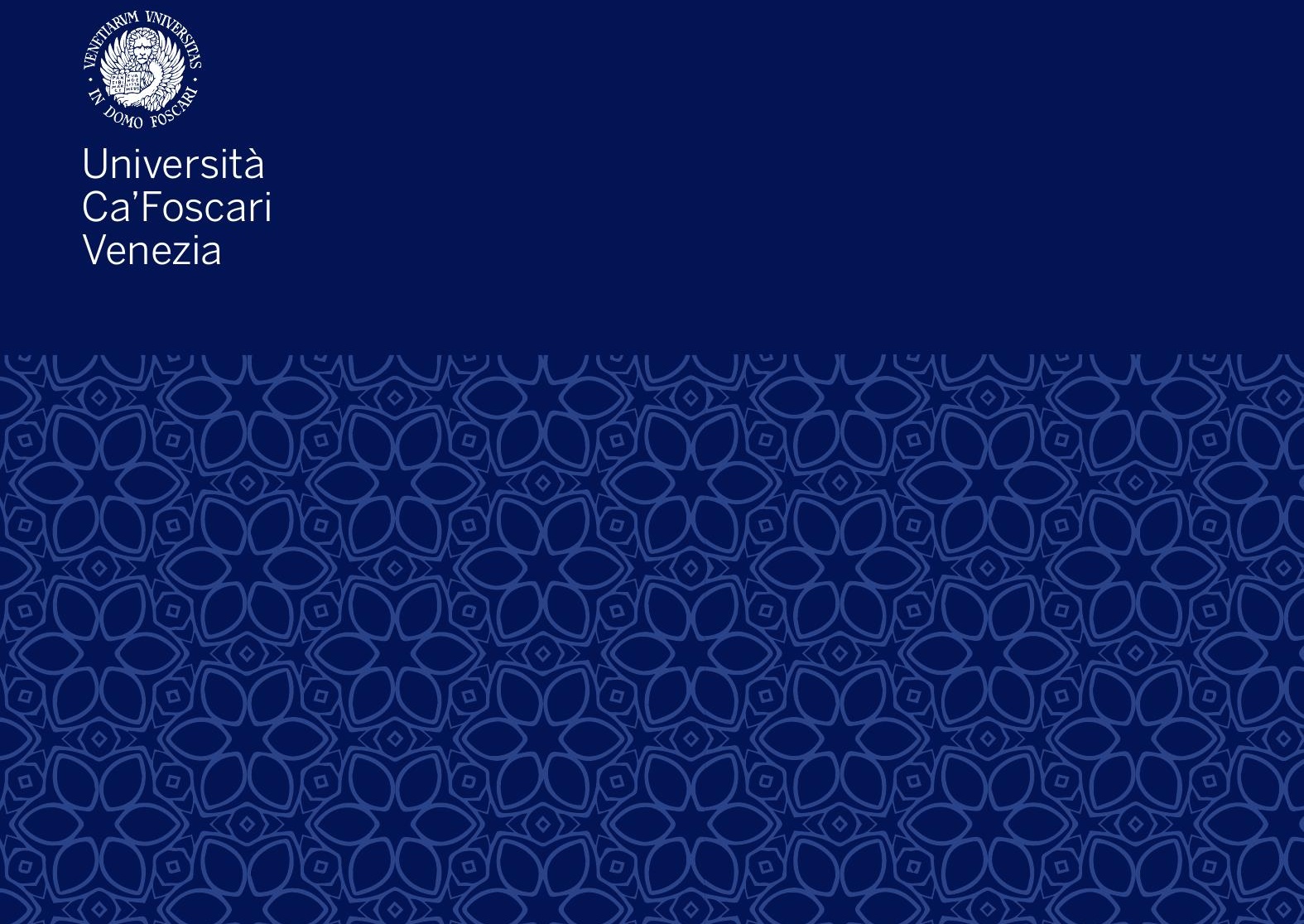